Portland State University

PDXScholar

Spring 5-14-2013

\title{
Characterization of Mesoscopic Fluid Films for Applications in SPM Imaging and Fabrication of Nanostructures on Responsive Materials
}

Xiaohua Wang

Portland State University

Follow this and additional works at: https://pdxscholar.library.pdx.edu/open_access_etds

Part of the Fluid Dynamics Commons, and the Other Physics Commons Let us know how access to this document benefits you.

Recommended Citation

Wang, Xiaohua, "Characterization of Mesoscopic Fluid Films for Applications in SPM Imaging and Fabrication of Nanostructures on Responsive Materials" (2013). Dissertations and Theses. Paper 1068. https://doi.org/10.15760/etd.1068

This Dissertation is brought to you for free and open access. It has been accepted for inclusion in Dissertations and Theses by an authorized administrator of PDXScholar. Please contact us if we can make this document more accessible: pdxscholar@pdx.edu. 
Characterization of Mesoscopic Fluid Films for Applications in SPM Imaging and Fabrication of Nanostructures on Responsive Materials

by

Xiaohua Wang

A dissertation submitted in partial fulfillment of the requirements for the degree of

\author{
Doctor of Philosophy \\ in \\ Applied Physics
}

Dissertation Committee:

Andres H. La Rosa, Chair

John L. Freeouf

Raj Solanki

Shankar B. Rananavare

Bin Jiang

Portland State University

2013 


\begin{abstract}
This dissertation focuses on characterization of the mesoscopic fluid film, testing its behavior in different application scenarios, including its role in near-field scanning probe microscopy imaging, contribution to the phononic mechanism in nanotribology phenomena, utilizing it as a natural environment in the study of carbohydrate-protein interactions, and harnessing it as bridge to transport ions in the fabrication of nanostructures on responsive polymer materials.

Due to their high resolution and versatile applications in a variety of fields, the family of scanning probe microscopy (SPM) has found widespread acceptance as an analytical and fabrication tool. However, the working mechanism of SPM that allows maintaining the probe-sample distance constant is still controversial. At the heart the problem is a lack of precise knowledge about the nature of the probe-sample interaction. One key factor is the presence of a mesoscopic fluid-like layer that naturally forms at any surface at ambient condition in which most SPMs are operated. Its mesoscopic nature ( $20 \mathrm{~nm}$ in thickness) results in extraordinary behavior compared to the properties of bulk liquid. For example, the effective shear viscosity of confined mesoscopic fluids is enhanced, and viscoelastic relaxation times are prolonged. Despite the wide use of SPM techniques in ambient air, the basis of their working mechanisms is still not well understood.

The probe-sample interaction is monitored using a combination of tuning-fork based shear force microscopy and our recently developed near-field acoustic technique. To characterize the mesoscopic fluid film a series of experiments are performed under different conditions in order to explore the benefits of having extra probing (acoustic)
\end{abstract}


technique in addition to the shear-force approach. The presence of mesoscopic fluid layers as a natural environment enables the detection of protein-carbohydrate interactions. We demonstrated the capability of our shear-force/acoustic technique to monitor the rupture of chemical bonds between carbohydrate and protein pairs. Finally, we present fabrication of nanostructures via electric-field assisted dip-pen nanolithography by exploiting the responsive feature of a particular class of polymers, where the mesoscopic fluid layer also plays an important role in pattern creation. 


\section{Acknowledgements}

I wish to express my warm and sincere thanks to my advisor Prof. Andres H. La Rosa. His broad knowledge and logical way of thinking have been of great value for me. The present dissertation would not have been possible without his understanding, encouraging and personal supervision.

I want to thank my committee members: Prof. John L. Freeouf, Prof. Raj Solanki, Prof. Shankar B. Rananavare and Prof. Bin Jiang for their generous guidance.

I would like to express special thanks to Prof. Mingdi Yan from Department of Chemistry at University of Massachusetts Lowell for her kind efforts on providing valuable suggestions and help.

I would like thank my current labmates: Rodolfo Fernandez, Elia Zegarra, and Bret Comnes. It is a pleasure to work with them, and discuss about our research. All of my coworkers offered me lots of help, as well as valuable suggestions to my works. I also want to thank Suji Uppalapati, and Sailaja Chada for providing samples for my studies.

I owe my loving thanks to my husband Dr. Xin Wang and my parents. Without their encouragement and great helps it would have been impossible for me to finish this work. 


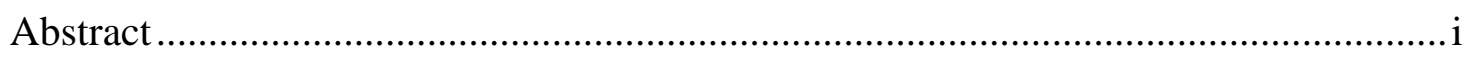

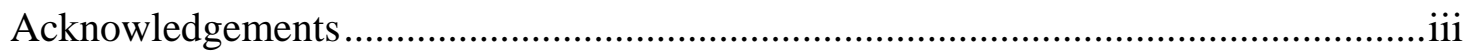

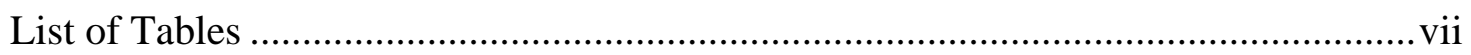

List of Figures ...........................................................................................................viii

Chapter 1. Toward the Development of Shear-Force/Acoustic Near-Field Microscopy .

\section{1}

1.1 Brief Introduction of Scanning Probe Microscopy …………............................... 1

1.2 Scanning Tunneling Microscopy ........................................................................ 2

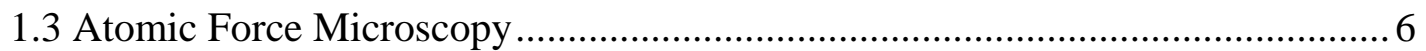

1.4 Near-Field Scanning Optical Microscopy ……………....................................... 10

1.5 Shear-Force/Acoustic Near-Field Optical Microscopy ….................................... 14

Chapter 2. Surface and Interfacial Phenomena Related to Scanning Probe Microscopy . 16

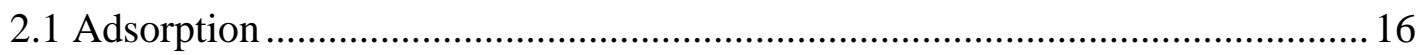

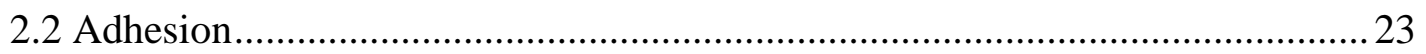

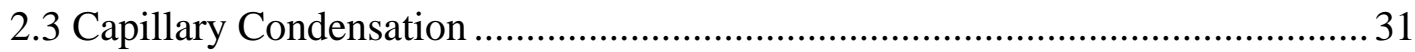

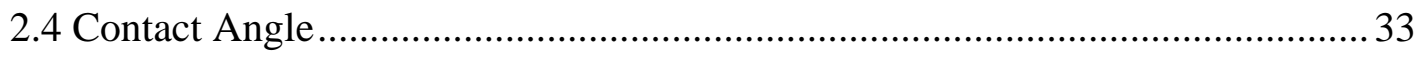

2.5 Hydrophobic/Hydrophilic Interactions.................................................................. 37

Chapter 3. Design of Shear-Force/Acoustic Near-Field Microscopy ............................. 39

3.1 The Quartz Tuning Fork................................................................................. 39 


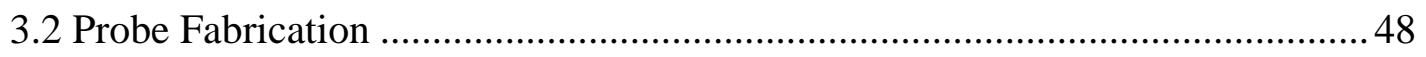

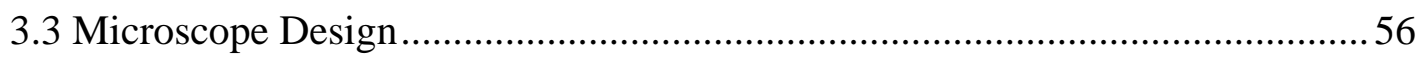

3.4 Detection Electronics .................................................................................. 58

Chapter 4. Field-Programmable Gate Array in Distance Regulation .............................. 60

4.1 Introduction of Field-Programmable Gate Array ............................................... 60

4.2 Precise Z-Axis Position Control via FPGA-DAQ Card.......................................62

4.3 Noise Level of Analog Outputs on FPGA-DAQ Card .........................................65

Chapter 5. Characterization of Mesoscopic Fluid Films via Shear-Force/Acoustic Near-

Field Microscopy

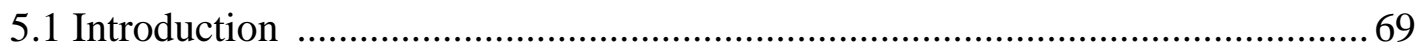

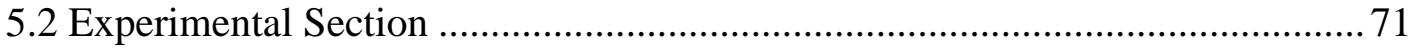

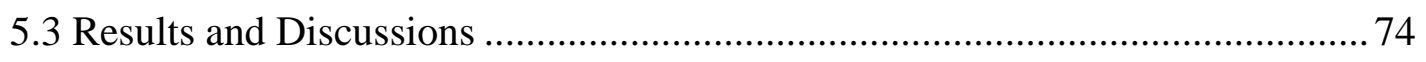

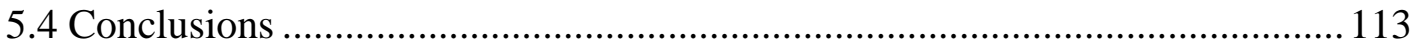

Chapter 6. Monitoring of Probe-Sample Mechanical Contact via Current Detection in

Shear-Force/Acoustic Near-Field Microscopy ......................................................... 116

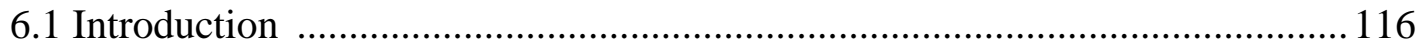

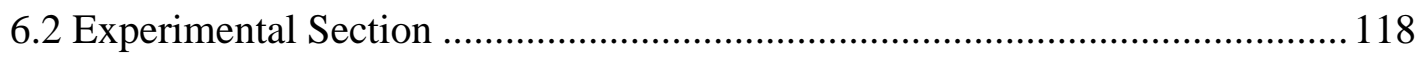

6.3 Results and Discussions ............................................................................ 123

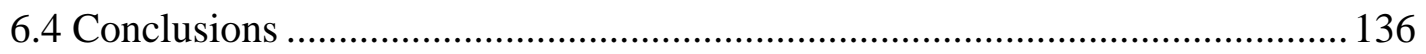

Chapter 7. Acoustic Detection with Probes Immersed in Liquid Droplets.................. 139

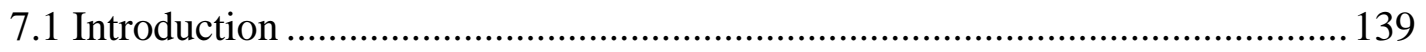

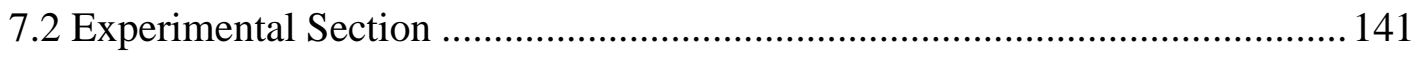


7.3 Results and Discussions

7.4 Conclusions .

Chapter 8. Detection of Carbohydrate-Protein Interactions via Shear-Force/Acoustic

Near-Field Microscopy.................................................................................... 156

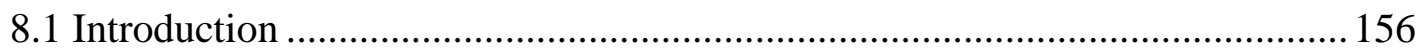

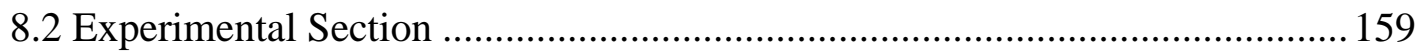

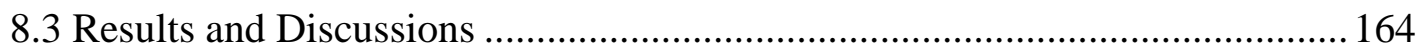

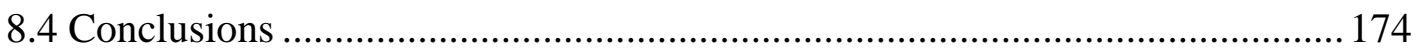

Chapter 9. Electric Field-Assisted Dip-Pen Nanolithography on P4VP Thin Films

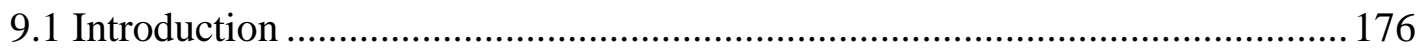

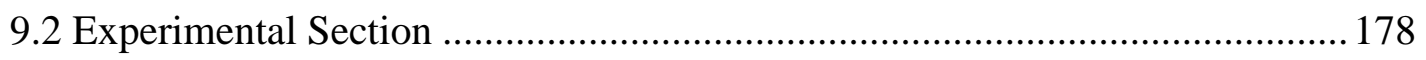

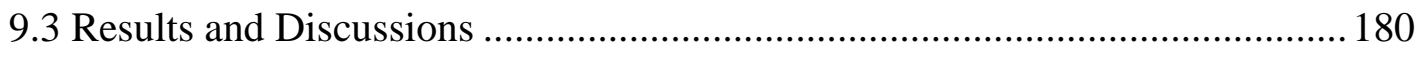

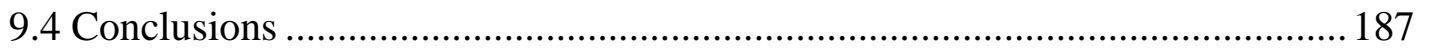

Chapter 10. Final Conclusions ................................................................................ 189

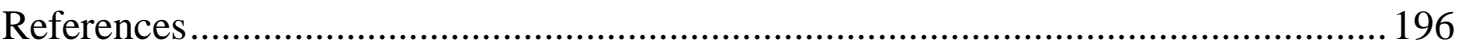




\section{List of Tables}

Table 4.1 Values of resistors and capacitor used in the low-pass filter........................ 68 


\section{List of Figures}

Figure 1.1 A schematic view of the design of scanning tunneling microscopy ............ 4

Figure 1.2 A sketch of the operating principle of atomic force microscopy ................ 8

Figure 1.3 A schematic diagram of one specific configuration of near-field scanning

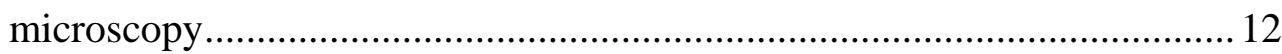

Figure 2.1 Diagram used for deriving the Young-Laplace equation ............................2 21

Figure 2.2 Definitions of interfacial tensions at different interfaces ......................... 25

Figure 2.3 Definitions of (a) the work of adhesion and (b) the work of cohesion........ 26

Figure 2.4 (a) Elastic sphere on rigid surface in the absence (Hertz) and presence (JKR) of adhesion. (b) The adhesion force necessary to separate solids from contact according to the JKR theory. (c) Neck formation according to the DMT theory

Figure 2.5 Two spherical particles in contact with liquid meniscus 33

Figure 2.6 (a) Liquid drop with circular contact area on a planar solid surface spreads an infinitesimal amount. (b-d) The various degrees of wetting: complete wetting $\left(\theta=0^{\circ}\right)$, partial wetting $\left(\theta<90^{\circ}\right)$, and nonwetting $\left(\theta>90^{\circ}\right)$

Figure 3.1 (a) Mechanical model of the TF resonator as a damped harmonic oscillator. (b) Equivalent electrical model of the TF oscillator as a series RLC circuit. (c) Sketch of the quartz TF drawn by Karrai and Grober $^{1}$

Figure 3.2 (a) Current measurement of an opened TF as a function of frequency. The resonance is followed by the antiresonance. (b) Definition of the quality factor based the resonance curve. $\Delta f$ is the FWHM of the waveform 44 
Figure 3.3 The equivalent circuit of the TF in electrical driving mode 46

Figure 3.4 (a) Comparison of the measured (open circle) and calculated (solid line) amplitude and phase of a TF in electrical driving mode. (b) The resulting converted curve reflecting the true harmonic motion of the same TF. 48

Figure 3.5 Sketch of the electrochemical etching station and the scanning electron microscope (SEM) image of the gold tip using DC etching method........... 50

Figure 3.6 The etching current intensity monitored during the etching procedure of gold wire (left), and the etching process of tungsten wire (right) 52

Figure 3.7 Sketch of the electrochemical etching station and the optical image (1000X magnification) of the gold tip using AC etching method ..... 54

Figure 3.8 (a) Optical images of the same glass fiber before and after a 2.5-hour etching. (b) The plot of fiber diameter vs etching time shows the resulting fiber diameter decreases upon increasing etching time...................................56

Figure 3.9 The key components of a microscope stage .......................................... 58

Figure 4.1 Strategy used to attain long range of motion with high resolution.............. 63

Figure 4.2 Schematic of the summing amplifier and corresponding voltage signal on each

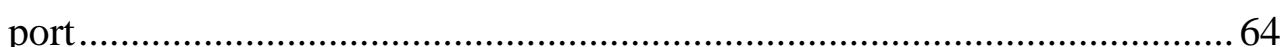

Figure 4.3 Schematic of low-pass filter used to diminish the unwanted noise from the analog outputs of FPGA-DAQ card 66

Figure 5.1 Schematic of the experimental setup used to detect the TF signal along with the acoustic signal as the gold tip approaches to and withdraws from the sample surface 72 
Figure 5.2 Five independent trials of approach (upper) and retraction (lower) measurements preformed on the same testing site. Different colors represent different trials 76

Figure 5.3 A summary of approach (upper) and retraction (lower) measurements preformed on five different testing sites. Different colors represent the tip being placed at different locations. Each curve is an average of five independent trials. 77

Figure 5.4 Scanning electron microscope images of a chubby gold tip (left) and a sharp gold tip (right). The scale bar represents $1 \mu \mathrm{m}$. 78

Figure 5.5 Approach (a) and retraction (b) curves of TF (blue) and acoustic (green) signals obtained with the use of a chubby tip and a bare silicon wafer (humidity: 25\%). Insertion: The detail of the TF signal at point B 81

Figure 5.6 Approach (a) and retraction (b) curves of TF (purple) and acoustic (red) signals obtained with the use of a sharp tip and a bare silicon wafer (humidity: $25 \%)$ 83

Figure 5.7 Approach (upper) and retraction (lower) curves of TF (light blue) and acoustic (light green) signals obtained with the use of a chubby tip and a bare silicon wafer (humidity: 55\%) 84

Figure 5.8 Approach (up) and retraction (lower) curves of TF (light purple) and acoustic (light pink) signals obtained with the use of a sharp tip and a bare silicon wafer (humidity: 55\%). 84 
Figure 5.9 Approach curves of TF (upper) and acoustic (lower) signals obtained using the chubby tip at R.H.=25\% (blue) and R.H. $=55 \%$ (light blue) 86

Figure 5.10 Retraction curves of TF (upper) and acoustic (lower) signals obtained using the chubby tip at R.H.=25\% (green) and R.H.=55\% (light green)..........86

Figure 5.11 Approach curves of TF (upper) and acoustic (lower) signals obtained using the sharp tip at R.H.=25\% (purple) and R.H.=55\% (light purple) 87

Figure 5.12 Retraction curves of TF (upper) and acoustic (lower) signals obtained using the sharp tip at R.H.=25\% (red) and R.H.=55\% (light pink) 87

Figure 5.13 Approach/retraction curves of the TF and acoustic signals on silicon substrate cleaned using Piranha solution: (a) curves obtained immediately after the Piranha treatment; (b) curves obtained on the same surface, $24 \mathrm{~h}$ after the cleaning 90

Figure 5.14 Scheme of experimental procedure to prepare H-terminated Si surfaces. Contact angle measurements on the corresponding surfaces (lower) show the change in hydrophobic-hydrophilic nature of the surface 92

Figure 5.15 Approach/retraction curves of the TF and acoustic signals on silicon substrate treated by aqueous HF solution: (a) curves obtained immediately after the HF treatment; (b) curves obtained on the same surface, 24h after the etching....

Figure 5.16 Approach/retraction curves of the TF and acoustic signals on silicon substrate cleaned with $\mathrm{O}_{2}$ plasma: (a) curves obtained immediately after the plasma 
etching; (b) curves obtained on the same surface, 24h after the plasma etching

Figure 5.17 Approach (a and b) and retraction (c and d) curves of TF and acoustic signals obtained on bare silicon (light blue and light green) and polymer coated silicon (blue and green) substrate 98

Figure 5.18 Scheme of experimental procedure for preparing hydrophobic and hydrophilic surfaces on the same Si substrate. 100

Figure 5.19 Results of contact angle measurements on the same Si substrate covered by PS and PEOX monolayer on each half, respectively. 100

Figure 5.20 Approach (a) and retraction (b) curves of TF (black and gray) and acoustic (blue and light blue) signals obtained on the PS film coated side with the use of a gold tip (humidity: 35\% 40\%) 103

Figure 5.21 Approach (a) and retraction (b) curves of TF (black and gray) and acoustic (green and light green) signals obtained on the PEOX layer covered side by employing the same gold tip as used on the PS coated side (humidity: $35 \% \sim 40 \%)$ 103

Figure 5.22 Approach curves of the TF (a) and acoustic (b) signals obtained on the PScoated (blue) and PEOX-coated (green) silicon wafer; and retraction curves of the TF (c) and acoustic (d) signals obtained on the PS-coated (light blue) and PEOX-coated (light green) silicon wafer. The experiment was performed by using the same tip at R.H. $=35 \% \sim 40 \%$. (e) The acoustic 
approach/retraction curves were re-drawn by separating the PS surface from the PEOX surface 107

Figure 5.23 Approach (a and b) and retraction (c and d) curves of TF and acoustic signals obtained on the gold surface using the same gold tip with a constant speed of approach (2 nm/s) but various speeds of retraction: $2 \mathrm{~nm} / \mathrm{s}$ (black), $4 \mathrm{~nm} / \mathrm{s}$ (red), $6 \mathrm{~nm} / \mathrm{s}$ (blue), and $12 \mathrm{~nm} / \mathrm{s}$ (green). Insertions: the approach/retraction curves of the TF (a) and acoustic (b) signals measured by moving the tip at the same speed of $2 \mathrm{~nm} / \mathrm{s}$. 109

Figure 5.24 (a) Scheme of experimental procedure to obtain approach/retraction curves from the bare glass and gold-coated surfaces. (b) AFM image shows the thickness of Au coating is $\sim 40 \mathrm{~nm}$. (c) The TF and acoustic signals measured on the glass (left) and gold surface (right). The amount of the decrease in the TF signal approximates to the same value, whereas the maximum value of the acoustic signal on the gold surface is greater than that on the glass surface.

Figure 6.1 Schematic of experimental setups used for detecting contact current, along with the TF and the acoustic signals. In configuration (a), a short gold tip is electrically connected to the TF electrode and thereby the TF driving voltage is used to bias the tip. In configuration (b), a long gold tip is mounted on the TF and the bias is applied through an extra Cu wire soldered to the tip.........122

Figure 6.2 A series of experiments were carried out to gradually land a gold tip on a gold surface. The resulting approach/retraction curves of the TF signal, the acoustic 
response and the probe-sample current were obtained by setting different set points in turn: (a) 90\%; (b) 80\%; (c) 60\%; (d) 40\%; and (e) $26 \%$ 126

Figure 6.3 Approach curves of the TF, the AS, and the probe-sample current signals obtained using the same gold tip: (a) if the tip was carefully adjusted, current signal was observed when the set point of the approach process was set to be 26\%; (b) if the tip was tilted on purpose, current signal occurred earlier when the set point was $43 \%$. The term $d_{o}$ represents the distance between the very end of the tip and the hard surface. As the tip is driven to oscillate, the term $d_{e}$ represents the effective (shortest) distance between the vibrating tip and the surface. For a given $d_{o}$, if tip is perpendicular to the surface $d_{e} \approx d_{o}$; if the tip is tilted $d_{e}<d_{o}$ 129

Figure 6.4 A typical approach/retraction curve obtained by concurrently measuring the TF signal, the acoustic response and the probe-sample current (the set point was 24\%): (a) approach and (b) retraction curves plotted by choosing the origin of $\mathrm{X}$ axis as the position where the approach process completes or the retraction process started. (c) The same result is re-drawn by selecting a new origin of $\mathrm{X}$ axis (the position where the TF amplitude drops to 95\% of its maximum) in order to avoid a misunderstanding in locating the actual hard surface. The upper scheme shows a possible explanation for the probe-sample interaction in different regions 132

Figure 6.5 (a) Approach curve of the TF, acoustic and current signals obtained using the experimental setup mentioned in Figure 6.1a. (b) Approach curve of the same 
signals measured using the experimental configuration described in Figure

$6.1 \mathrm{~b}$ 136

Figure 7.1 Schematic of experimental setup used to detect the TF and acoustic signals when the cleaved fiber is laterally oscillating in a droplet of glycerol aqueous solution $(\sim 5 \mu \mathrm{L})$ placed on a mica disk 142

Figure 7.2 Typical vibration spectra of the TF (a) and acoustic (b) signals at immersion depth $=40 \mu \mathrm{m}$ (black), $120 \mu \mathrm{m}$ (red), $200 \mu \mathrm{m}$ (blue) and $280 \mu \mathrm{m}$ (green), as well as the converted TF spectra which represent the mechanical behavior of a harmonic oscillator (c) 145

Figure 7.3 Resonance frequency shift of the TF (left) and acoustic (right) signals as a function of penetration depth using the same probe in liquids with different viscosities. 147

Figure 7.4 Resonance frequency shift of the TF (left) and acoustic (right) signals as a function of penetration depth using probes with various diameters in the same liquid 147

Figure 7.5 Change in amplitude of resonance peaks of the TF (left) and acoustic (right) signals as a function of penetration depth using the same probe in liquids with different viscosities 149

Figure 7.6 Change in amplitude of resonance peaks of the TF (left) and acoustic (right) spectra at various penetration depths using probes with different diameters in the same liquid. 150 
Figure 7.7 Plots of measured amplitude of the TF (top) and acoustic (middle) signals at resonance as a function of driving voltage, at different submersion depths, respectively 151

Figure 7.8 Vibration spectra of the TF and acoustic signals obtained by exciting the same fiber to oscillate in drops of distilled water with different volumes 153

Figure 7.9 Recording TF frequency response when the fiber oscillates and stays at a fixed depth in distilled water. The time interval between two traces next to each other is $1 \mathrm{~min}$, and the entire process lasts $\sim 5 \mathrm{~min}$. The details of resonance position are shown in the inset 154

Figure 8.1 Schematic of experimental procedure to prepare the mannose-terminated gold tip (left) and the protein-immobilized silicon wafer (right). 160

Figure 8.2 Schematic of experimental setup used to detect the TF signal along with the acoustic signal as the gold tip approaches towards and subsquently retracts from the sample surface 163

Figure 8.3 Typical approach (a) and retraction (b) curves of TF and acoustic signals plotted as a function of the tip’s vertical displacement. (c) Schematic demonstration of the interaction between the mannose-terminated tip and the Con A-terminated surface. 167

Figure 8.4 Repeatability of acoustic retraction traces representing mannose-Con A unbinding events 
Figure 8.5 Control experiments performed on unspecific binding surfaces: approach (a) and retraction (b) curves of TF and acoustic signals for PFPA-coated surface; and approach (c) and retraction (d) curves for PNA-coated substrate ...... 173

Figure 9.1 Different experimental settings used for patterning features on P4VP polymer films: A tip was inked with the acidic buffer and (a) not biased, (b) negatively biased, (c) positively biased. (d) An uncoated tip while applying a positive bias 181

Figure 9.2 (a-e) Topographic images of features drawn using the tip coated with acidic ink. The applied bias voltages $(\mathrm{V})$, contact forces $(\mu \mathrm{N})$ and measured heights of selected lines (nm) are the following: (a) 5, 1, 13; (b) 3, 1, 6; (c) 0, 1, 3; (d) 5, 0.8, 8; (e) 5, 0.5, 3. (f) A line feature with $60 \mathrm{~nm}$ wide. (g) A pattern of the letters: PSU. Image sizes: $8 \mu \mathrm{m} \times 8 \mu \mathrm{m}$ for (a-e); $5 \mu \mathrm{m} \times 5 \mu \mathrm{m}$ for (f-g). (h) Schematic showing that the swelling of P4VP film originates from the protonation of the pyridine units. (i) The pattern height (nm) v.s. bias voltages (V) at various fixed contact forces. (j) The pattern height (nm) v.s. contact forces under different constant bias voltages 182

Figure 9.3 Topographic images show the removal of the dot pattern at the center: (a) Initially, the dot height was $12 \mathrm{~nm}$; (b) After the first erasing performance, the height decreased to $7 \mathrm{~nm}$; (c) After the second erasing performance, the remaining height was only $3 \mathrm{~nm}$. Line profiles of (d), (e), and (f) correspond to the features selected in (a), (b), and (c), respectively. 184 


\section{Chapter 1. Toward the Development of Shear-Force/Acoustic Near-Field Microscopy}

\subsection{Brief Introduction of Scanning Probe Microscopy}

Scanning probe microscopy (SPM) comprises a family of techniques that allow measurement of surface topography with atomic scale resolution. Since the invention of SPMs, for example, the scanning tunneling microscope (STM) in $1982^{1}$, and the atomic force microscope (AFM) in $1986^{2}$, they have become powerful tools which could be used not only to determine surface structures but to characterize surface properties. There are an increasing number of publications concerning technical advances of SPMs, and their applications are greatly extended. SPMs are now routinely used in industries and academic labs with a diverse range of goals, for example, material manipulation, structure creation, and the development of new concepts in electronics ${ }^{3}$. The general principle of operation of a SPM is based on the use of a probe and the ability to regulate its position in relation to the surface of interest with atomic scale precision during scanning process. Compared to optical microscopes and electron microscopes, the attractive feature of SPMs is they are capable of providing real space images at nanometer scale in all three dimensions $\mathrm{X}, \mathrm{Y}$ and $\mathrm{Z}$.

The family of SPM techniques is very diverse. Various members differ in the nature of the probes that are employed and the mechanisms that control the image formation. For instance, STMs use a sharp conducting tip as the probe and the control parameters of operation are the tunneling current and/or the voltage, whereas AFMs employ a microfabricated cantilever with a sharp tip as the transducer to sense the force between 
tip and sample for scanning control. In near-field scanning optical microscopes

(NSOMs) ${ }^{4}$, both metal coated tapered optical fibers with a small opening at the apex and sharpened homogeneous metal tips are used in terms of types of NSOM. Tip-sample distance regulation relies on the detection of shear forces resulting from the interaction between the end of tip and the sample of interest as the tip scans along the surface. The aim of this chapter is to provide an understanding of the basic operation principles of commonly used SPM techniques---STM, AFM and NSOM, as well as an introduction of the shear-force/acoustic near-field microscopy developed in our lab.

\subsection{Scanning Tunneling Microscopy}

There is no doubt that the birth of scanning tunneling microscope (STM) ${ }^{1}$ is landmark event in the history of nanoscience and nanotechnology. By successfully combining vacuum tunneling and scanning capability, the STM provides an insight into a novel field of Angstrom unit scale research and technology. The inventors, Binning and Rohrer, were awarded the Nobel Prize in physics for the STM in 1986.

Quantum tunneling refers to the quantum mechanical phenomenon where an electron in one side of a potential energy barrier, with energy smaller than the barrier height, has a nonvanishing probability of passing the barrier and tunneling into the other side. The STM uses the same physical process of tunneling in its operating principle ${ }^{5}$. When an atomically sharpened tip is brought into proximity of a sample surface, i.e., on the order of a few nanometers, under a bias voltage across the gap, a small electric current starts to flow from the surface of one material to the surface of another even when the surfaces are 
not in contact with each other. The magnitude of the tunneling current is extremely sensitive to the distance between surfaces. Qualitatively, at low voltage and temperature, the tunneling current $I$ can be expressed as:

$$
I \propto e^{-2 \kappa d} .
$$

Here $d$ is the separation between two surfaces---to be precise, it is the distance from the nucleus of the tip apex to the nucleus of surface atoms; and $\kappa$ is a material-dependent constant, in the case of vacuum tunneling, it is related to the effective local work function. Since the tunneling current has a strong exponential dependence on the tip-sample distance, it is suitable to be used as the control parameter in STM experiments to yield topographic images with atomic scale resolution in the vertical direction with respect to the sample surface.

From an instrumental point of view, the STM is mainly consists of the following parts:

(1) An atomically sharpened metal tip and a sample placed to be faced to the tip apex.

(2) Positioning devices that allow three-dimensional tip motion with atomic resolution to control the separation between the tip and the sample, and scan the tip over an area to be observed.

(3) A current amplifier to measure the tunneling current and a feedback circuit to maintain a constant current.

(4) A computer system that enables data acquisition, data analysis, data processing and data visualization. 
In addition, a good vibration isolation system, together with an ultrahigh vacuum (UHV) chamber can effectively prevent undesired disturbances and keep the tip and the sample clean, resulting in true atomic-resolution images.

Figure 1.1 shows a schematic diagram of an STM system. A conducting tip is attached at a piezoelectric scanner. An appropriate tip material is chosen in terms of the specific application. Under UHV conditions, tungsten tips are most widely used for STM experiments. For STM performance in air, it is favorable to select an inert material, e.g. platinum or gold, for the tip. Sensor tips are prepared via several different methods, such as electrochemical etching/polishing, ion milling, mechanical cutting and field evaporation ${ }^{6-8}$. These methods are sometimes combined, leading to novel tip preparation approaches.

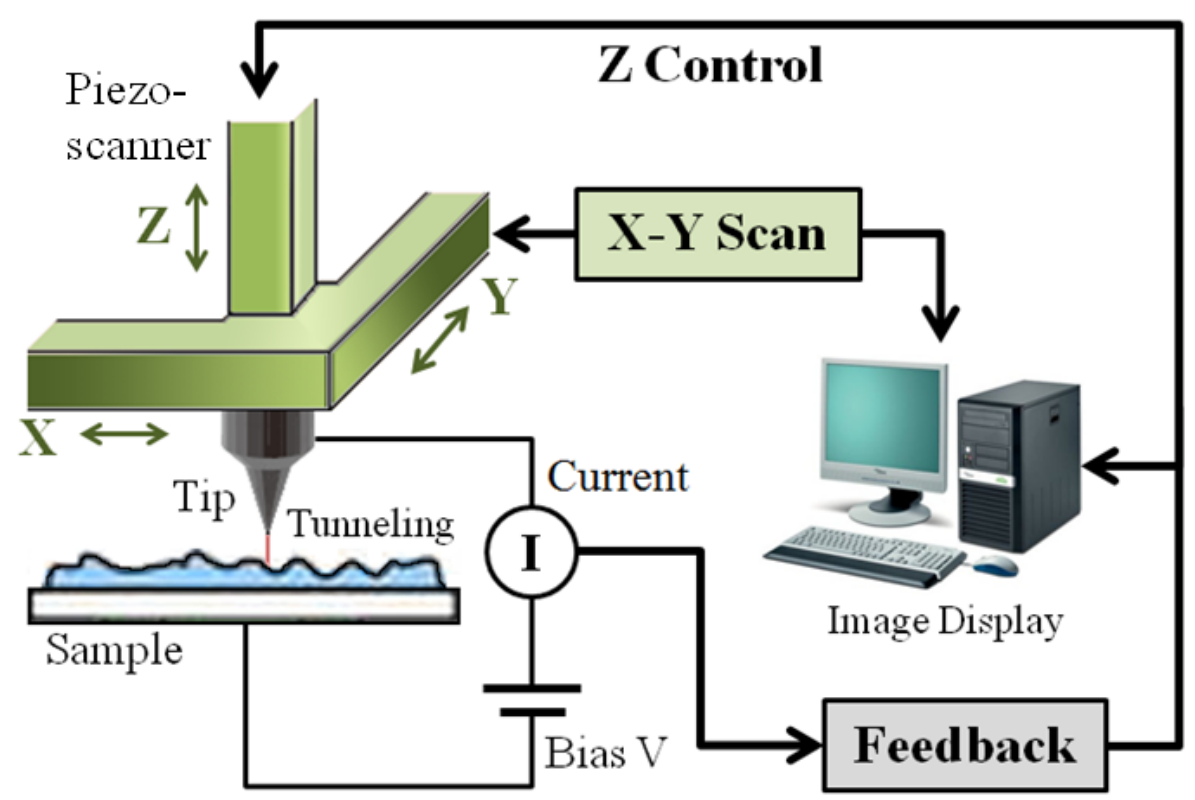

Figure 1.1: A schematic view of the design of scanning tunneling microscopy. 
When the STM scanning is started, the tip is brought closer to the sample from a farther separation. A bias voltage ( $2 \mathrm{mV} 2 \mathrm{~V}$ ) is applied between tip and sample, and the current produced by tunneling of electrons through the gap before the two physically touch is measured. The feedback loop controls the height of the tip with the objective of maintaining a constant tunneling current. The lateral scan and the vertical position of tip are usually achieved by deformations of the piezoelectric element. Typically, the STM can be operated in either the constant current mode or the constant height mode ${ }^{1,9}$. In the constant current mode, since the tip-sample separation increased or decreased due to variations in the sample topography, the feedback control attempts to maintain the current constant in a manner that actuates the positioning unit away from or toward the sample. Therefore, the vertical displacement of tip reflects the change in the surface height, yielding a map of the surface topography. Alternatively, in the constant height mode, the feedback mechanism responds only rapidly enough to keep the average current constant. The tip can be scanned across the surface at nearly constant height and constant voltage, and thereby the local variations in current due to the tip passing over surface features are acquired.

Samples to be investigated with the STM must be electrically conductive to some degree to allow a few nanoamperes of current to flow from the bias voltage source to the area to be scanned. Nonconductive samples are generally coated with a thin layer of a conductive material to facilitate imaging. The bias voltage and the tunneling current depend on the sample; the different atomic species within a sample may produce different tunneling currents for a given bias voltage. In reality scanning with a constant current is 
not actually a representation of the atomic topography, but more correctly a map of the electronic states of the substrate. Owing to the ability to provide information on the local electronic levels, the STM can be beneficially used to measure the electronic properties of the sample with high spatial resolution.

\subsection{Atomic Force Microscopy}

The STM has already been a powerful tool for studying surface constructions. However, the use of STM is limited to conductors and semiconductors. Interrogation of insulators at the nanoscale was unavailable until 1986, when Binnig, Quate, and Gerber invented the atomic force microscope $(\mathrm{AFM})^{2}$. Like the STM, the AFM relies on a scanning technique to yield high resolution, three-dimensional images of conducting as well as non-conducting surfaces. Unlike the STM, sample conductivity is no longer a requirement in AFM experiments. The AFM has become the most versatile tool that permits the study of almost whole classes of important materials including insulators and large band-gap semiconductors. The 2000 U.S. National Nanotechnology Initiative noted that "STMs, AFMs, and near-field microscopes provide the eyes and fingers required for nanostructure, manipulation, and measurement”.

The AFM is capable of sensing extremely small forces. A flexible cantilever with a sharp tip provides the means of detecting the forces present between the AFM probe surface and a sample surface, typically in the range of $10^{-7} \sim 10^{-12} \mathrm{~N}$, and thereby it is the essential element to significantly affect the spatial resolution of the AFM. At present, microfabricated $\mathrm{Si}$ and/or $\mathrm{Si}_{3} \mathrm{~N}_{4}$ cantilevers are widely used. In practice, to perform the 
AFM experiment, the tip attached to the free end of the cantilever with a known spring constant is brought into close proximity to a surface to be observed. The fixed end of the cantilever is supported by a carrier, which is attached to a dither piezo. The dither piezo provides a way to vibrate the carrier. The cantilever bends vertically in response to attractive and/or repulsive interaction forces ${ }^{10}$, and the motion of the cantilever influenced by these forces is observed and quantified by a detection system. Similar to the STM, laterally and vertically positioning of the sample is achieved by a piezoelectric scanner during scanning.

A schematic diagram of an AFM setup is shown in Figure 1.2. The conventional method for measuring the cantilever deflection is using the optical beam deflection technique. In the AFM, a laser beam is focused on the cantilever and reflected from the cantilever backside into a position-sensitive quadrant photodetector (PSPD). When the sample is moved laterally under the tip, features on the surface cause the cantilever to deflect. The cantilever deflection changes the direction of the reflected laser beam, which, in turn, changes the intensity difference between the top and bottom (left and right) sets of PSPD. The laser beam deflection method in fact reads out changes in the small angle that the incident beam makes with the cantilever surface, which is linearly proportional to the cantilever bending. The length of the reflected laser path amplifies this deflection. This method is believed to be the most sensitive, reliable and easily implemented detection approach. 


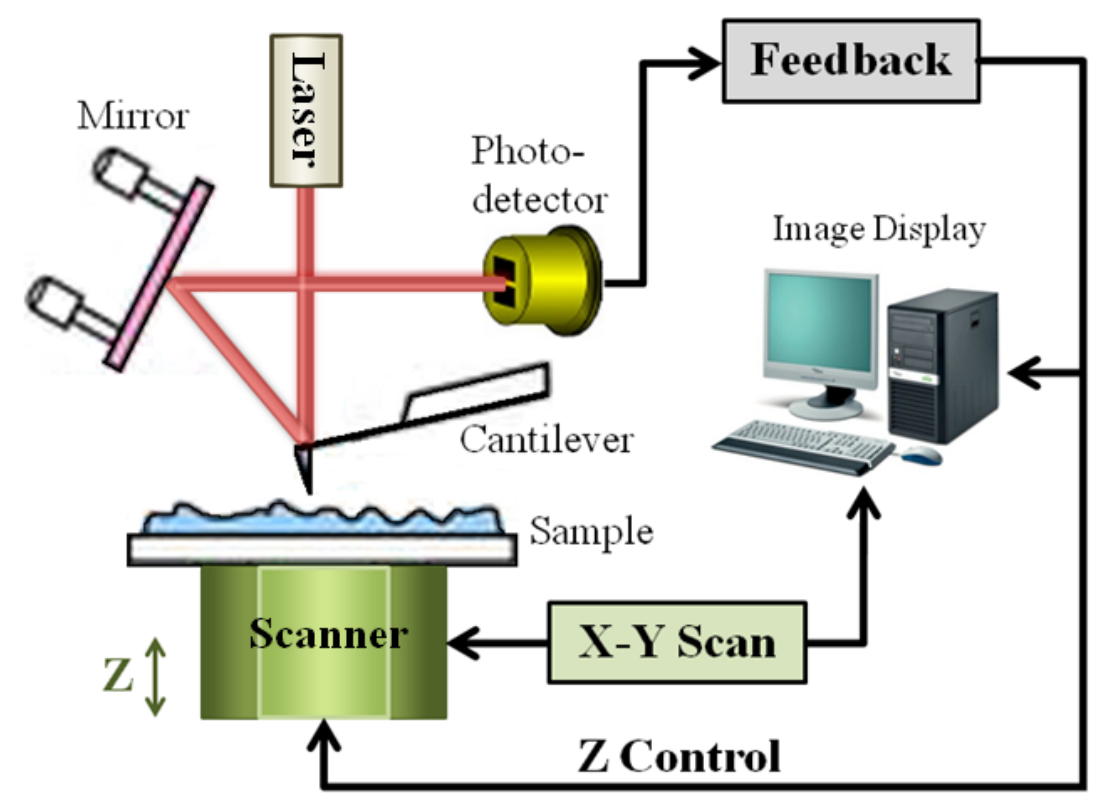

Figure 1.2: A sketch of the operating principle of atomic force microscopy.

The cantilever deflection varies under the impact of the forces acting on the tip. Therefore, surface topographic information is stored during a lateral scan of the sample while simultaneously probing the separation-dependent force or force gradient between tip and sample. The measurement of tip-sample interaction is either recorded directly, or used as a control parameter for a feedback circuit that maintains the force or force gradient at a constant value by moving the sample vertically to compensate variations. There are two types of imaging modes in AFM:

(1) In the static mode, also referred to as contact mode, the AFM tip is always brought in contact with the sample surface. Under the “contact” regime, a very weak repulsive force experienced by the tip results in an upward deflection of the cantilever from its equilibrium position. The force is detected from the cantilever deflection and, consequently, surface structure is obtained. 
(2) In the dynamic mode, the cantilever is driven at a fixed frequency near resonance. When the tip is far from the surface, the oscillation amplitude of the vibrating cantilever is held constant. When the tip is close to the surface, the vibration amplitude decreases. The force gradient is sensed to form a map of surface topography ${ }^{11}$. In detail, the dynamic mode can be classified into two types: tapping mode and noncontact mode. In the tapping mode, the tip is periodically in contact with the surface, and surface structure is gained from the change of the oscillation amplitude and/or phase, which is caused by cyclic repulsive contact between tip and sample with loss of the energy stored in the cantilever. In the noncontact mode, the mechanical contact of tip and sample does not occur, and topographic image is obtained from the change of the oscillation amplitude and/or resonant frequency, which is resulted from interaction forces, i.e., van de Waals forces, attracting the tip to the surface.

The contact mode has a high possibility that the strong repulsion will damage the sample surface and/or the tip apex. So, the tapping and noncontact modes are widely used because they are more gentle than the contact mode.

The AFM experiments can be performed in a variety of environments: ambient air, various controlled atmosphere, liquids, vacuum, at low temperatures and high temperatures ${ }^{12,13}$. Imaging in liquid allows the study of live biological samples and it also eliminates water capillary forces present in ambient air at the tip-sample interface. Low temperature imaging is useful for the study of biological and organic materials and the study of low-temperature phenomena. Moreover, nanoscale science and technology are 
strongly driven by AFMs which allow investigation of surfaces down to the atomic scale. AFMs have been used to study the physics and chemistry of probe-sample interactions, as well as quantitatively analyze tribological, electronic, magnetic, biological, and chemical surfaces. Aside from its ability to image and visualize the specimen, the AFM is of practical importance for nanofabrication and nanomachining ${ }^{14,15}$.

\subsection{Near-Field Scanning Optical Microscopy}

After the invention of the STM as an extreme example of a super-resolution microscope, STM technology also triggered increasing effort in the development of other scanning probe microscopy techniques. Near-field scanning optical microscopy (NSOM) is a new proximal probe technique providing the ability to image sample surfaces with

resolution in the 10 100-nm regime ${ }^{4,16}$. It is known that classical optics is concerned with the far-field regime, which is defined as any distance that is greater than one wavelength being used away. The spatial resolution achieved in classical optical microscopy is limited by the wavelength of light source, also referred to as the Abbe limit. This limit arises from diffraction of electromagnetic waves interacting with an object to be imaged. On the other hand, by operating a microscope in the near-field regime, the Abbe resolution limit can easily be surpassed and therefore sub-wavelength features of the object to be imaged can be retrieved.

In the NSOM an optical probe is scanned in the direct vicinity of a sample surface to be imaged. The probe illuminates locally the surface and the intensity of the light transmitted through the sample is recorded. Consequently, optical information with 
respect to the surface is accessible point by point. Spatial resolution of the SNOM is afforded primarily by the probe size and the probe-to-sample separation rather than the wavelength of light. To achieve a high lateral resolution, the probe diameter has to be of the order of nanometer (generally, 10 200 nm), and the probe has to be maintained at a distance of less than $10 \mathrm{~nm}$ from the sample surface.

Figure 1.3 demonstrates a design of NSOM apparatus. There are several NSOM configurations that can be utilized in terms of sample properties or working environments. One particular NSOM configuration is usually referred to as illumination mode $\mathrm{NSOM}^{17,18}$, where the NSOM probe acts as a localized light source, with the transmitted light or emitted fluorescence being collected by a far-field optic. Alternatively, successful examples of imaging have been reported with collection mode $\mathrm{NSOM}^{19,20}$, in which the NSOM probe acts as a localized light collector rather than an illuminator, and the farfield optic is used to excite the sample. 


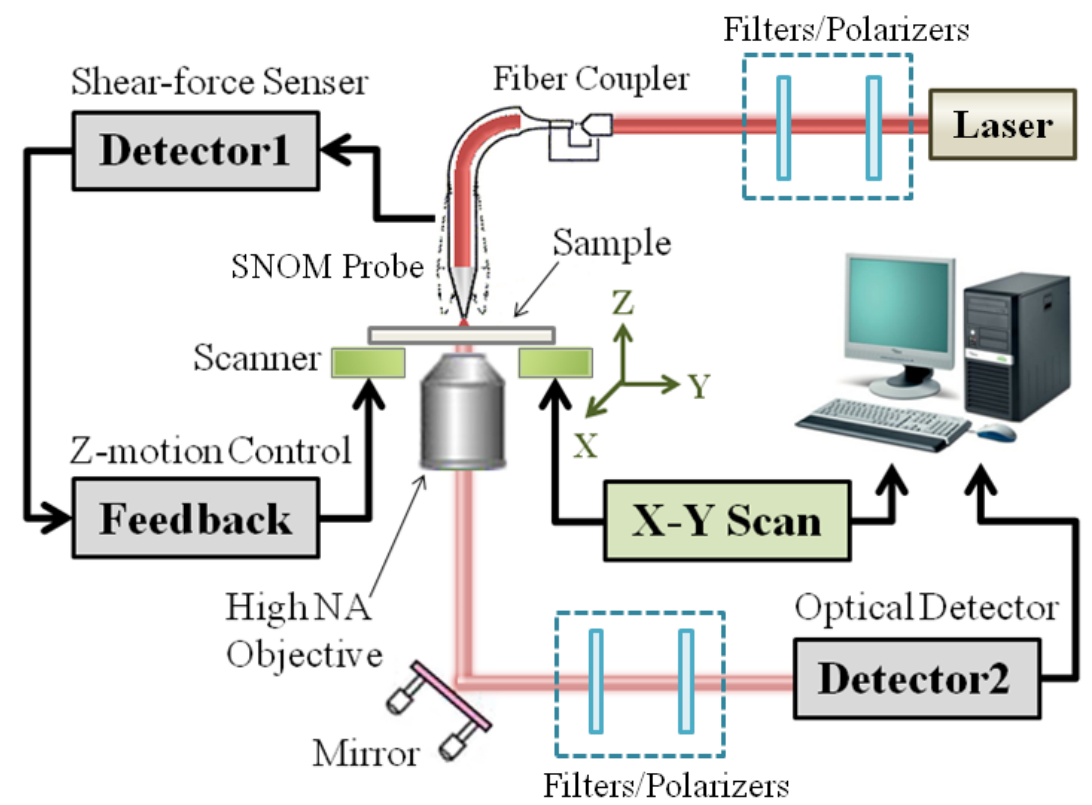

Figure 1.3: A schematic diagram of one specific configuration of near-field scanning optical microscopy.

Like classical optical microscopy, the NSOM can be performed in transmission or in reflection ${ }^{21-23}$, the choice of which is determined by the sample of interest. In transmission NSOM, a thin sample is excited and light passing through it is collected using short working distance, high numerical aperture (NA) optics. For opaque samples, reflection NSOM is utilized in which the NSOM probe illuminates the sample and light is collected with a long working distance collection lens by either mounting the lens directly above the sample and probe, or placing it at an angle to the sample and probe.

The NSOM can also be classified into aperture-type and apertureless-type based on probe structure. In aperture-type NSOM, the probe is fabricated by a tapered optical fiber tip coated with a thin reflective metal layer, leaving a nanometer sized aperture at the apex ${ }^{4}$. As an alternative option, sharpened micropipettes coated with a thin metal film can serve as probes as well ${ }^{18}$. The aperture probes can either be used to deliver the nanoscale 
light spot, or they can be used for both excitation and collection. In apertureless-type NSOM, the probe is formed by a sharpened metal tip ${ }^{24,25}$. Illumination with the polarization parallel to the tip apex can create large amount of oscillating surface charges which lead to a greatly enhanced electric field laterally confined to the tip apex. When the apertureless probe is used for imaging, spatial resolution is purely dependent on the tip geometry. The field enhancement effect can also contribute to the detection of extremely weak signals associated Raman spectroscopy ${ }^{26}$.

Sample scanning is accomplished using piezoelectric scanner, similar to other scanning probe microscopes such as STM and AFM. Since NSOM probes are positioned and held within a few nanometers of the sample surface during scanning, tip-sample distance regulation plays a central role in the NSOM operation. One of the most commonly adopted feedback mechanisms is the shear-force technique ${ }^{27}$. In the shearforce method, the NSOM probe is mounted on to a small piezo element, such as dither piezo or quartz tuning fork, and vibrated in a motion parallel to the surface at one of its mechanical resonances. As the tip approaches to and about few tens of nanometers above the sample, the amplitude of the vibration is damped due to the existence of shear forces between tip and sample. The amplitude, an analog to the interaction forces in AFM experiments, can be monitored by various methods and used in a feedback circuit for tipto-sample distance control during imaging.

NSOM techniques have been proven to important characterization tools in materials science. The NSOM has be to image a variety of samples, and the same contrast mechanisms known in classical optical microscopy are available using NSOM 
approaches, such as transmission, reflection, emission, absorption, polarization and spectroscopy. The NSOM is well suited for semiconductor applications owing to its ability to noninvasively measure surface topography, defect content, and carrier lifetime variations ${ }^{20,28}$. Other examples of applications using NSOM can also be found in single molecule studies, thin film analysis, and probing biological samples ${ }^{29-34}$.

\subsection{Shear-Force/Acoustic Near-Field Microscopy}

Shear forces were first utilized as a feedback mechanism in NSOM techniques ${ }^{27,35}$ and, subsequently, shear-force scanning microscopy ${ }^{36}$ was born as a by-product of the NSOM. As described above, in a shear-force scanning microscope, a tip is dithered laterally at one of its mechanical resonances. The amplitude of the vibration is kept low. As the tip approaches the sample surface, the dithering tip will experience damping due to the presence of shear forces between the tip and the sample. It is found that in ambient conditions, this drop in amplitude normally occurs over a range of tens of nanometers from the surface. The shear-force scanning microscope is an instrument that uses the effect of shear-force interactions to generate a feedback signal in the distance regulation during a scan.

To date, quartz tuning forks (QTFs) are being used as force sensors and QTF-based shear-force scanning microscopes are widely accepted. QTFs were initially introduced into the field of SPM by Gunther et al. ${ }^{37}$, and later by Karrai and Grober ${ }^{35}$. Owing to the piezoelectric effect native to quartz crystal, the measurement of QTFs' oscillation amplitude is achieved by evaluating the change in an electrical signal correlated to the 
applied forces. The implementation of QTFs greatly simplifies force measurement schemes without losing sensitivity too much. QTFs have others advantages such as high amplitude and phase sensitivities, high quality factor, high stiffness, and no hazard to photosensitive materials. Furthermore, QTFs have been successfully demonstrated under various conditions ${ }^{38,39}$.

Acoustics imaging based on transmission and reflection of acoustic waves has been used for a long time in characterization of the mechanical properties of materials, such as elasticity measurements and flaw detection ${ }^{37,40,41}$. In a classical scanning acoustic microscopy, an acoustic wave is focused at the surface of or inside the investigated target. After interaction with the sample, the transmitted or reflected acoustic waves are detected via ultrasonic transducers. Inhomogeneities or defects can also be visualized even if they are in a certain penetration depth inside the sample. Herein, we developed a novel near field scanning probe microscopy named Shear-force/Acoustic Near-field Microscopy (SANM) by incorporating an acoustic sensor to a shear force microscope. As a combination of shear force and scanning acoustics microscopes, the SANM is expected to be able to simultaneously provide the shear-force and acoustic interpretations regarding the surface structures as well as subsurface features of a sample. 


\section{Chapter 2. Surface and Interfacial Phenomena Related to Scanning Probe Microscopy}

\subsection{Adsorption}

Adsorption plays an important role towards attaining both a theoretical and a practical understanding of surface and interfacial phenomena. It can be simply referred to as the accumulation of a substance at an interface. The material in the adsorbed state is called adsorbate. The substance to be adsorbed (before it is on the surface) is called adsorptive. The substance, onto which adsorption occurs, is called adsorbent.

Adsorption takes place at any type of interfaces. However, interfaces consisting of only liquids and vapors display slightly simpler adsorption features than those that have solid surfaces involved in. The reason is that at liquid surfaces, the complications arisen from surface heterogeneities and inhomogeneities can be neglected. A freshly prepared solid surface will often be of quite high surface energy so that there will exist strong driving forces for reducing the excess surface energy. Unless solid surfaces are in ultrahigh vacuum (UHV), under common laboratorial conditions all gas molecules will adsorb to solid surfaces below a critical temperature because of attractive intermolecular forces. How much of a material adsorbed to an interface is usually dependent upon the temperature.

In general, when adsorption is dominated by physical interaction rather than chemical bonding, the process is referred to as physisorption; if the adsorption energy is of the order of chemical binding energies, this process is termed chemisorption. Physisorption is a process in which only nonspecific interactions are involved. In molecular physics, the 
electronic structure of the molecule or atom is hardly perturbed upon absorption. The corresponding mechanism is van der Waals forces. In the absence of complicated factors (i.e., capillary condensation), physisorption has no activation energy. Adsorption equilibrium is rapidly established and this process is reversible. The adsorbate is relatively free to diffuse on the surface. Furthermore, physisorption will be a multilayer process: that is, the amount of adsorbate particles which can be adsorbed onto a surface will not be limited by the available solid surface area, but the particles can stack up to several molecular thicknesses in a pseudo-liquid assembly.

In contrast, chemisorption is a process that often involves specific binding sites between the solid surface and the adsorbate atoms or molecules, which resembles the formation of covalent or ionic bonds. From a molecular viewpoint, chemisorption is accompanied by a rearrangement of the electronic shells of the adsorbate atoms or molecules, where the electronic structure of the bonding partners is strongly perturbed, and new hybrid orbitals are formed. In the case of ionic bonding, there may be charge transfer from one partner to the other. Chemisorbed molecules must overcome an activation energy, and they usually do not diffuse on the surface. Often the molecules first physisorb to the surface and in a second, much slower, step the chemical bond is established, forming a monomolecular chemisorbed layer. Molecule desorption may not be accessible.

There are several theoretical principles that are fundamental for understanding all adsorption phenomena. Herein, we will focus on the Gibbs adsorption equation, the Young-Laplace equation, and the Kelvin equation, which are introduced in turn. 


\subsubsection{The Gibbs Adsorption Equation}

The classical Gibbs adsorption equation is applicable to the case where one interface is present between two coexisting surface phases. It describes changes in the surface tension with variances in the concentration of a species in contact with a surface ${ }^{42}$.

To define the state of a bulk phase $\alpha$, it is essential to specify the values of several variables associated with the state. Those variables include the temperature $T^{\alpha}$, its volume $V^{\alpha}$, and the system pressure $P^{\alpha}$. In terms of the Helmholtz free energy $A$, the system can be specified by (with constant $P$ ):

$$
d A^{\alpha}=-S^{\alpha} d T^{\alpha}-P^{\alpha} d V^{\alpha}+\sum \mu_{i}^{\alpha} d N_{i}^{\alpha}
$$

where $S^{\alpha}$ is the entropy, $N_{i}^{\alpha}$ and $\mu_{i}^{\alpha}$ are the amounts and chemical potentials of species $i$. For a system of two phases, a similar equation can be written for the second phase $\beta$. At equilibrium, the two phases will have the same temperature $T$, the same pressure $P$, and the same chemical potential $\mu$, for all components. The $\alpha-\beta$ interface present is considered to be a third phase $\sigma$. Therefore, the total free energy can be expressed as:

$$
A^{T}=A^{\alpha}+A^{\beta}+A^{\sigma}
$$

where $A^{\sigma}$ is the interfacial Helmholtz free energy. Analogous to Eq. (2.1.1), the derivative of the interfacial free energy is given by:

$$
d A^{\sigma}=-S^{\sigma} d T+\sum \mu_{i} d N_{i}^{\sigma}+\gamma d A_{S}
$$

where $\gamma$ is the surface or interfacial tension between $\alpha$ and $\beta, A_{s}$ is the interfacial area, and $\mu_{i}$ has the same value as that in the bulk phase. According to the Gibbs surface model, the surface tension is related to an interface, which behaves mechanically as a 
membrane stretched uniformly and isotropically by a force which is the same at all points and in all directions. We integrate Eq. (2.1.3) while keeping the intrinsic parameters $T$, $\mu_{i}$, and $\gamma$ constant:

$$
A^{\sigma}=\sum \mu_{i} N_{i}^{\sigma}+\gamma A_{S}
$$

This integration is allowed because it represents a feasible process, e.g., through simply increasing the surface area of the system.

To derive the Gibbs adsorption equation, we start with Eqs. (2.1.3) and (2.1.4). At equilibrium, taking differential of Eq. (2.1.4) leads to:

$$
d A^{\sigma}=\sum \mu_{i} d N_{i}^{\sigma}+\sum N_{i}^{\sigma} d \mu_{i}+\gamma d A_{s}+A_{s} d \gamma
$$

Equating this to Eq. (2.1.3) then results in:

$$
A_{S} d \gamma+S^{\sigma} d T+\sum N_{i}^{\sigma} d \mu_{i}=0
$$

At constant temperature, Eq. (2.1.6) can be simplified to:

$$
-d \gamma=\sum N_{i}^{\sigma} d \mu_{i} / A_{S}
$$

By defining a new term like a surface concentration, $\Gamma_{i}=N_{i}^{\sigma} / A_{S}$, called interfacial excess concentration of $i$, the Gibbs adsorption equation becomes:

$$
-d \gamma=\sum \Gamma_{i} d \mu_{i}
$$

For dilute solutions containing one non-dissociating surfactant, a more general form of the Gibbs adsorption equation is:

$$
d \gamma=-R T \Gamma_{2}^{(1)} d \ln C_{2}
$$

in which 2 designates the solute dissolved in bulk phase 1 , and $C_{2}$ denotes the molar concentration of surfactant in bulk. The practical applicability of this equation is that the 
relative adsorption of a material at an interface can be determined from measurement of the interfacial tension as a function of solute concentration:

$$
\Gamma_{2}^{(1)}=-\frac{1}{R T}\left(\frac{d \gamma}{d \ln C_{2}}\right)
$$

\subsubsection{The Young-Laplace Equation}

The Young-Laplace equation defines the equilibrium condition for the pressure

difference over a curved interface in terms of surface tension and curvature ${ }^{43}$. Since many practical applications involve surfaces and interfaces with high degree of curvature, it is important to understand the effect of curvature on interfacial properties.

To derive the expression of the pressure difference across a curves surface, we pick up a small part of a liquid surface (Figure 2.1). A point $\mathrm{X}$ is selected and a line is drawn on which all points are the same distance $l$ away from X. Then, on this line we take two cuts (AXB and CXD) that are perpendicular to each other. At point $\mathrm{B}$, the surface tension pulls with a force $\gamma d l$; the vertical force is $\gamma d l \sin \alpha$; and for small surface area we have $\sin \alpha \approx l / R_{1}$, where $R_{1}$ is the radius of curvature along AXB. Therefore, the sum of the four vertical components at points A, B, C, and D is:

$$
\gamma \cdot d l \cdot\left(\frac{2 l}{R_{1}}+\frac{2 l}{R_{2}}\right)=\gamma \cdot d l \cdot 2 d \cdot\left(\frac{1}{R_{1}}+\frac{1}{R_{2}}\right)
$$

Integrating Eq. (2.1.11) over the borderline gives the total vertical force, caused by the surface tension. In equilibrium, this vertical force must be compensated by an equal force in the opposite direction. The balanced force is caused by an increased pressure $\Delta P$ on the concave side. Equating both forces leads to: 


$$
\begin{gathered}
\pi l^{2} \cdot \gamma \cdot\left(\frac{1}{R_{1}}+\frac{1}{R_{2}}\right)=\pi l^{2} \cdot \Delta P \\
\Delta P=\gamma \cdot\left(\frac{1}{R_{1}}+\frac{1}{R_{2}}\right)
\end{gathered}
$$

Eq. (2.1.13) is the Young-Laplace equation, which is valid for any liquid surfaces. In circumstances where the surface tension is uniform from point to point, the pressure difference will depend only on the curvature of the interface. Due to the presence of a pressure difference, the liquid will flow from high to low pressure until the difference is decreased and finally vanish. In particular, for a spherical surface $R_{1}=R_{2}=r$, the equation simplifies to $\Delta P=2 \gamma / r$. In a plane surface where $R_{1}=R_{2}=\infty, \Delta P=0$.

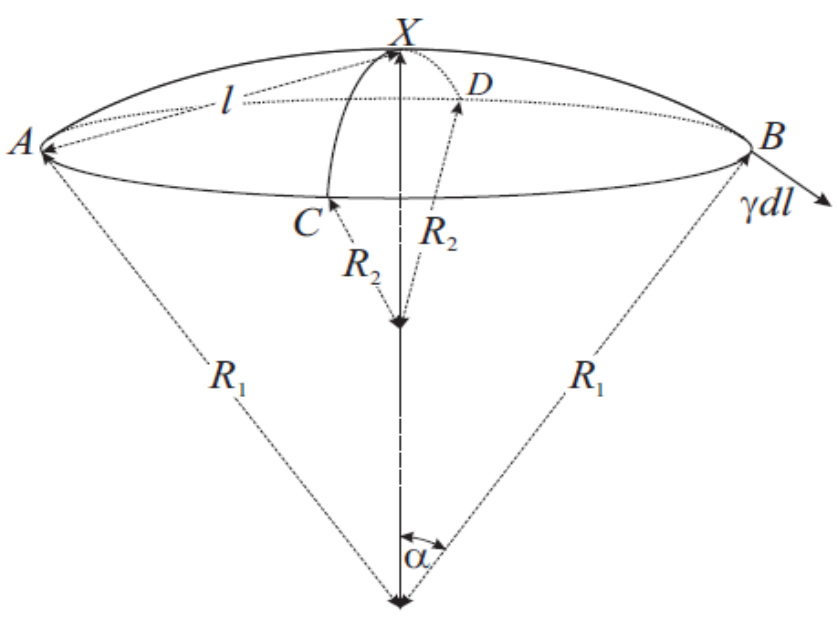

Figure 2.1: Diagram used for deriving the Young-Laplace equation.

\subsubsection{The Kelvin Equation}

Like the Young-Laplace equation, the Kelvin equation is based on the thermodynamic principles and does not refer to a special material or special condition. 
The subject of the Kelvin equation is the vapor pressure of a liquid. It is known that when the liquid surface is curved, the vapor pressure changes. The Kelvin equation states how the vapor pressure depends on the curvature of the liquid ${ }^{44}$.

The cause for changes in vapor pressure is the Laplace pressure. For a very small drop of liquid in which there is a large surface to volume ratio, the vapor pressure is higher than that over a plane surface of equal area. The movement of liquid from a flat surface into a volume with a curved surface requires the input of energy into the system since the Gibbs free energy of the surface being curved increases. If the radius of a drop is increased by a factor $d r$, the surface area increases from $4 \pi r^{2}$ to $4 \pi(r+d r)^{2}$, the Gibbs free energy increases:

$$
\Delta G=\gamma \cdot 8 \pi r \cdot d r
$$

As same as the discussion in previous sections, $\gamma$ represents the surface tension of the adsorbed film. If during a process $d n$ moles of liquid are transferred from the flat phase with a vapor pressure of $P_{0}$ to a drop with vapor pressure $P_{0}^{K}$, the free energy increase is given by:

$$
\Delta G=R T \cdot \ln \left(P_{0}^{K} / P_{0}\right) \cdot d n
$$

where $R$ is the ideal gas constant, $T$ is the absolute temperature. Equating Eqs. (2.1.14) and (2.1.15) yields the expression known as the Kelvin equation:

$$
R T \cdot \ln \left(\frac{P_{0}^{K}}{P_{0}}\right)=\gamma V_{m} \cdot\left(\frac{1}{R_{1}}+\frac{1}{R_{2}}\right)
$$

$R_{1}$ and $R_{2}$ are the radii of curvature of the volume, $V_{m}$ is the molar volume of the liquid.

For a spherical drop of radius $r$, the equation can be simplified: 


$$
R T \cdot \ln \left(\frac{P_{0}^{K}}{P_{0}}\right)=\frac{2 \gamma V_{m}}{r} \quad \text { or } \quad P_{0}^{K}=P_{0} \cdot \exp \left(\frac{2 \gamma V_{m}}{r R T}\right)
$$

\subsection{Adhesion}

As a practical definition, adhesion is a state in which two bodies are held together by intimate interfacial contact in such a way the mechanical force or work can be applied across the interface without causing these two bodies to separate. Specifically, there are several mechanisms of adhesion used to explain why one material sticks to another. Thermodynamic adhesion is the term that applies to the "ideal" adhesion defined in terms of reversible work needed to separate two surfaces by overcoming the molecular interactions across the interface. Mechanical adhesion is the term that may be applied to the situation in which actual mechanical interlocking of microscopic asperities at the interface occurs over a significant portion of the contact area. Chemical adhesion refers to adhesion involving the formation of formal chemical bonds (covalent, electrostatic, or metallic) across an interface.

Before entering into the topic of adhesion, it is worth introducing several concepts related to adhesion, such as surface tension, interfacial tension, work of adhesion and work of cohesion. The main subject in this section concerns the adhesion force between solid objects. Basic theoretical models of contacts regarding microscopy techniques will be reviewed. 


\subsubsection{Surface Tension and Interfacial Tension}

The concept of surface tension was induced in previous sections; however, it is helpful to back up to interpret the concept of surface tension in detail. The surface tension is defined as the work required producing a new surface per unit area ${ }^{45}$. Under conditions of equilibrium at constant temperature and pressure, to increase the surface area by an amount $d A_{s}$ of a one-component system, the reversible work being done is given by:

$$
d W=\gamma \cdot d A_{S}
$$

The proportionality constant $\gamma$ in Eq. (2.2.1) is called surface tension. Eq. (2.2.1) can be compared with the reversible work needed to increase the volume at constant pressure, $P \cdot d V$. Therefore, the surface tension $\gamma$ can be considered as the two-dimensional analogue of the pressure $P$ while the volume change is replaced by the change in surface area. The unit of surface tension is either $\mathrm{J} / \mathrm{m}^{2}$ or $\mathrm{N} / \mathrm{m}$. For solids surface tension is commonly denoted by $\gamma_{S}$, and for liquid it is commonly denoted by $\gamma_{L}$.

On the molecular level, the surface tension $\gamma$ can be interpreted as the energy required to bring molecules from the bulk to the surface and to create new surface area. For molecules it is energetically favorable to be surrounded by other molecules. At the surface, however, molecules are only partially surrounded by other molecules and the number of adjacent molecules is smaller than in the bulk. In order to bring a molecule to the surface, work has to be done. Based on thermodynamics, the surface tension can also be defined as:

$$
\left.\gamma \equiv \frac{\partial A}{\partial A_{S}}\right|_{T, V, N_{i}} \quad \text { or }\left.\quad \gamma \equiv \frac{\partial G}{\partial A_{S}}\right|_{T, P, N_{i}}
$$


in which $A$ represents the Helmholtz free energy and $G$ is the Gibbs free energy. The former one states how the Helmholtz free energy changes when increasing the surface area while holding the temperature $T$, the total volume $V$, and the total numbers of all components $N_{i}$ constant. The later one is the increase in the Gibbs free energy per increase in surface area at constant $T, P$, and $N_{i}$.

The interfacial tension is a term similar to the surface tension in which two immiscible phases are involved ${ }^{46}$. When two bulk phases $\alpha$ and $\beta$ are in contact, the reversible work done or the free energy change in expanding their interfacial area by unit area is known as their interfacial tension $\gamma_{\alpha \beta}$. Figure 2.2 shows interfacial tensions at different interfaces. $\gamma_{L G}$ denotes the liquid-gas interfacial tension, $\gamma_{S L}$ denotes the solidliquid interfacial tension, and $\gamma_{S G}$ denotes the solid-gas interfacial tension, respectively. They will be present in next sections.

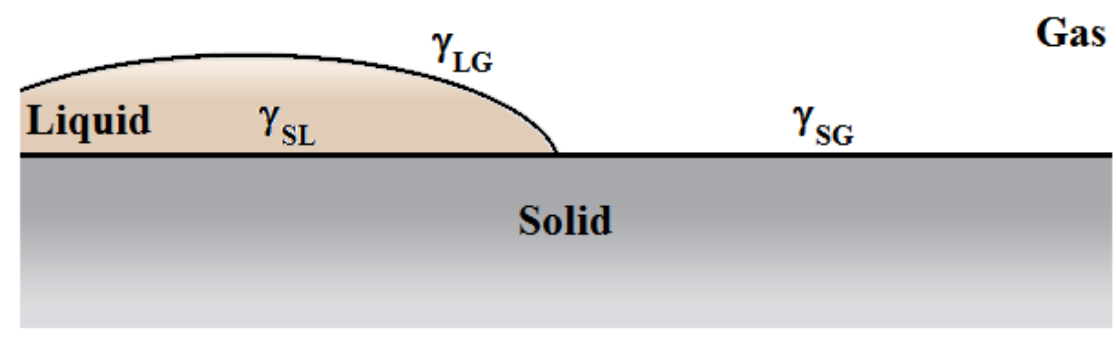

Figure 2.2: Definitions of interfacial tensions at different interfaces.

\subsubsection{Work of Adhesion and Work of Cohesion}

For two immiscible phases $\alpha$ and $\beta(\alpha \neq \beta)$, the work of adhesion $W_{A(\alpha \beta)}$ is defined as, the reversible work done or the free energy change, to separate unit area of interface 
between these two materials from contact to infinity in vacuum (Figure 2.3a) ${ }^{47}$. The work of adhesion is given by:

$$
W_{A(\alpha \beta)}=\gamma_{\alpha}+\gamma_{\beta}-\gamma_{\alpha \beta}
$$

where the subscripts refer to the two phases being separated, and the $\gamma$ 's are the respective surface or interfacial tensions.

For two identical phases $\alpha$ and $\beta(\alpha=\beta)$, analog to $W_{A(\alpha \beta)}$ is the work of cohesion $W_{C(\alpha \alpha)}$, defined as the reversible work required to separate two surfaces of unit area of the material (Figure 2.3b) ${ }^{47}$. It represents a minimum amount of work for the creation of two unit areas of fresh surface. The work of cohesion is expressed by:

$$
W_{C(\alpha \alpha)}=2 \gamma_{\alpha}
$$

Since all media attract each other in vacuum, $W_{A(\alpha \beta)}$ and $W_{C(\alpha \alpha)}$ are always positive.

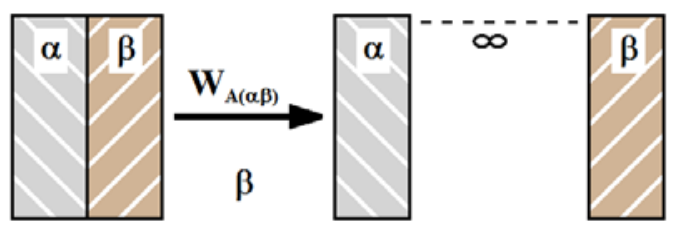

(a) Work of adhesion, $W_{A(\alpha \beta)}$

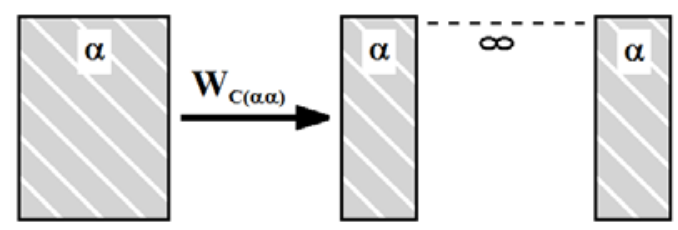

(b) Work of cohesion, $\mathrm{W}_{\mathrm{C}(\alpha \alpha)}$

Figure 2.3: Definitions of (a) the work of adhesion and (b) the work of cohesion.

In theory, the concept of thermodynamic adhesion applies equally well to liquid and solid phases. However, it does not take into consideration conditions before or after the formation of the interface, the presence of random flaws or defects in the system, or the bulk physical properties of the components. The complete expression for the work of 
adhesion between two phases with each phase completely saturated by the other, denoted by $\alpha(\beta)$ and $\beta(\alpha)$ is:

$$
W_{\alpha(\beta) \beta(\alpha)}=\gamma_{\alpha(\beta)}+\gamma_{\beta(\alpha)}-\gamma_{\alpha \beta}
$$

The maximum force required to separate unit area of interface, or the ideal adhesive strength of the interface $F_{a}$ can be estimated by ${ }^{48}$ :

$$
F_{a d}=1.03 W_{\alpha(\beta) \beta(\alpha)} / r_{0}
$$

where $r_{0}$ is the equilibrium distance of separation on the order of a few molecular diameters. Similar calculations can be made for cohesive strength as well.

\subsubsection{Adhesion Force Between Solid Surfaces: The Basic Theories of Contact}

In reality all solids have a finite elasticity. When real surfaces come into contact, they deform elastically under the influence of any externally applied load as well as the attractive interface forces that pull the two surfaces together. This results in a finite contact area even under zero external loads.

Heinrich Hertz was the first to lay the basis for the mechanical behavior of two elastic spheres (or a sphere and a plane surface) pressed together ${ }^{49}$. The standard Hertzian model considers two spheres with radii $R_{1}$ and $R_{2}$ in contact (or a sphere contacting a plane) (Figure 2.4a). The two solids are from materials with Youngs moduli $E_{1}$ and $E_{2}$, and with Poisson ratios $v_{1}$ and $v_{2}$. These spheres are pressed together by a load $F$ that can be the gravitational force or an applied force. The Hertzian model supposes that no surface forces such as van der Waals, adhesion and capillary forces act between the solids. 
According to this theory, the pressure between the spheres decreases as a quadratic function with the distance to the contact center. At the outer rim of the contact region with a radius $a$, the pressure drops to zero. A relationship between the contact radius $a$ and the load $F$ is obtained by integrating the pressure over the entire contact area:

$$
a^{3}=\frac{3 R^{*}}{4 E^{*}} \cdot F
$$

$R^{*}$ is the effective particle radius and $E^{*}$ is the reduced Youngs modulus expressed by:

$$
R^{*}=\frac{R_{1} \cdot R_{2}}{R_{1}+R_{2}} \quad \text { and } \quad \frac{1}{E^{*}}=\frac{1-v_{1}^{2}}{E_{1}}+\frac{1-v_{2}^{2}}{E_{2}}
$$

The contact radius also determines the indentation:

$$
\delta=\frac{a^{2}}{R^{*}}
$$

Hence a force-distance relationship is given by:

$$
F=\frac{4}{3} \cdot E^{*} \cdot \sqrt{R^{*} \delta^{3}}
$$

In fact surface forces such as van der Waals forces attract the two solids even for zero load. The theory developed by Johnson, Kendall, and Robert completed the Hertzian theory by taking account of adhesion forces ${ }^{50}$. Their model is called the JKR model. The JKR theory is valid when attractive surface forces are not negligible, and for soft materials such as polymers. It calculates the increase in contact area that results from the elastic bodies deforming to accommodate their mutual attraction. Similar to the Hertz theory, the JKR model considers the situation in which two elastic spheres of radii $R_{1}$ and $R_{2}$, with Youngs moduli $E_{1}$ and $E_{2}$, and with Poisson ratios $v_{1}$ and $v_{2}$, are brought into 
contact under a load F (Figure 2.4a). This is equivalent to a sphere-plane contact by considering one sphere to have an infinite radius. The energy gain per unit area is the work of cohesion, $W_{C}$, which is correlated with an energy loss of the system. At meantime, work must be done for the elastic deformation of the solids. With the help of the Hertz theory, this elastic deformation energy can be calculated, so that the real contact radius $a$ is given by:

$$
a^{3}=\frac{3 R^{*}}{4 E^{*}} \cdot\left(F+3 \pi W_{C} R^{*}+\sqrt{6 \pi W_{C} R^{*} F+\left(3 \pi W_{C} R^{*}\right)^{2}}\right.
$$

where $R^{*}$ and $E^{*}$ are as same as those in the Hertzian model. At zero applied load, there is a finite contact area given by:

$$
a_{0}=\left(\frac{9 \pi W_{C} R^{* 2}}{2 E^{*}}\right)^{1 / 3}
$$

Eq. (2.2.11) further implies that under small upward loads the solids still stick together until at some critical force the surfaces suddenly jump apart. The force necessary to separate two solids is termed the adhesion force or "pull-off” force (Figure 2.4b):

$$
F_{a d h}=\frac{3 \pi}{2} \cdot W_{C} R^{*}
$$

The cohesion work is usually expressed in terms of the surface tension of the solid $\gamma_{S}$ by Eq. (2.2.4). Substituting Eq. (2.2.4) into Eq. (2.2.13) leads to:

$$
F_{a d h}=3 \pi \gamma_{S} R^{*}
$$

The adhesion force increases linearly with the sphere radius. Surprisingly, it is independent of the elasticity of the materials. 


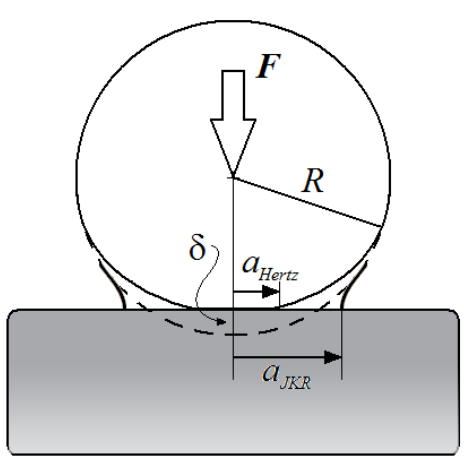

(a) Elastic sphere: adhesion

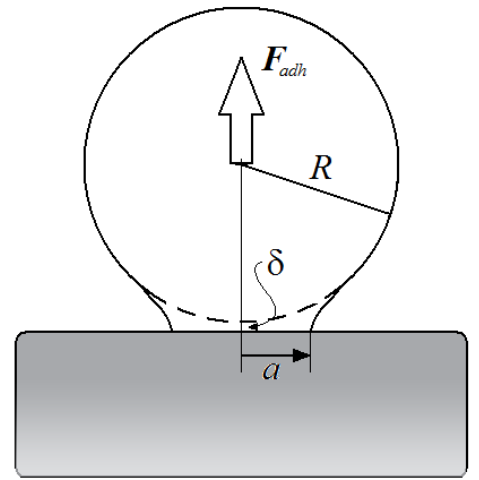

(b) Elastic sphere: pull-off

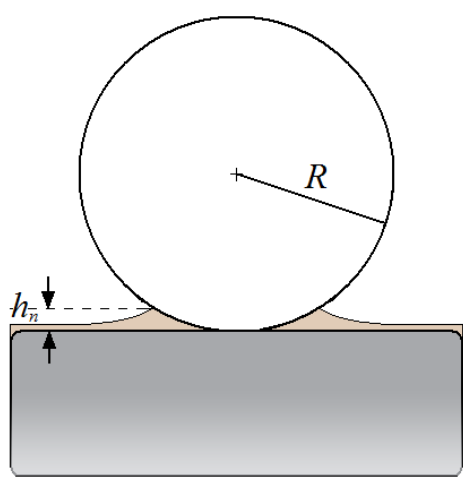

(c) Neck formation

Figure 2.4: (a) Elastic sphere on rigid surface in the absence (Hertz) and presence (JKR) of adhesion. (b) The adhesion force necessary to separate solids from contact according to the JKR theory. (c) Neck formation according to the DMT theory.

In the JKR theory it is assumed that surface forces are active only in the contact area. In reality surface forces are active also outside of direct contact. This is, for example, the case for van der Waals forces. Derjaguin, Muller, and Toporov took the effect of van der Waals forces outside the elastic contact area into account and developed the DMT model $^{51}$. The DMT theory applies to the case of small radii of curvature and weak adhesion forces. With this model, the contact radius $a$ can be written:

$$
a^{3}=\frac{3 R^{*}}{4 E^{*}} \cdot\left(F+F_{a d h}\right)
$$

where $R^{*}$ and $E^{*}$ have the same meaning as above, and $F_{a d h}$ is the adhesion force. In the DMT model it is given by:

$$
F_{a d h}=4 \pi \gamma_{S} R^{*}
$$

Exact analysis shows that the JKR model and the DMT model represent two extremes of the real situations ${ }^{52,53}$. For large, soft solids the JKR theory describes the situation 
realistically. For small, hard solids it is appropriate to use the DMT theory. A criterion, which model is to be used, results from the height of the neck (Figure 2.4c):

$$
h_{n}=\left(\frac{R^{*} \gamma_{S}^{2}}{E^{* 2}}\right)^{1 / 3}
$$

If the neck height is larger than some atomic distances, the JKR model is more favorable. With shorter neck heights the DMT model is more suitable. In addition, theories of contact such as the Hertzian and the JKR models assume that surfaces are perfectly smooth. However, real surfaces are rough. There is as yet no satisfactory theory for such real world situations.

\subsection{Capillary Condensation}

The formation of water films on surfaces of materials exposed to humid environments is the basis of many capillary phenomena. Capillary phenomena arise from differences in pressure across a system containing at least one liquid phase and another liquid, vapor, and/or solid phase. Such pressure differences may result from the presence of differences in curvature in the interface. The term "capillary condensation” tends to be defined as the condensation of vapor into capillaries or fine pores even for vapor pressures below the equilibrium vapor pressure of the liquid with a planar surface. Capillary condensation often strengthens the adhesion of fine particle. For example, as two spherical particles come into contact, liquid will spontaneously condense into the gag of the contact area. The meniscus is curved, and the Laplace pressure expressed by Eq. (2.1.13) in the liquid is negative. As a result, the particles attract each other. 
An important consequence of capillary condensation is the existence of the capillary force ${ }^{54}$, which is critical at the microscale. As described in the example given above, capillary forces arise from the formation of a concave meniscus around the contact, given rise to a negative Laplace pressure. Of interest for capillary forces is the contact between three phases: liquid, solid and gas. To calculate the capillary force, we consider a simple case where two spherical particles of identical radius $R_{p}$ are in contact (Figure 2.5). We further assume that the liquid wets the surfaces. The total radius of curvature of the liquid surface is:

$$
\frac{1}{R_{1}}+\frac{1}{R_{2}}=\frac{1}{x}-\frac{1}{r} \approx-\frac{1}{r} \quad(x \gg r)
$$

According to the Young-Laplace equation, the Laplace pressure in the liquid is:

$$
\Delta P=\frac{\gamma_{L}}{r}
$$

where $\Delta P$ is lower in the liquid than in the outer vapor phase. The pressure acts on a cross-sectional area $\pi x^{2}$ between the two surfaces leading to an attractive force of $\pi x^{2} \cdot \Delta P$. We can use Pythagoras' theorem to express $x^{2}$ in terms of $r$ :

$$
\begin{gathered}
\left(R_{p}+r\right)^{2}=(x+r)^{2}+R_{p}^{2} \\
2 r R_{p}=x^{2}+2 x r \approx x^{2} \quad(x \gg 2 r)
\end{gathered}
$$

Thus the attractive capillary force is:

$$
F=2 \pi \gamma_{L} R_{p}
$$

The capillary force depends only on the radius of the particles $R_{p}$ and the surface tension of the liquid $\gamma_{L}$. It is independent neither from the actual radius of curvature of the liquid 
surface nor from the vapor pressure. In reality, however, the force is often much smaller than the calculated value. This can be explained by the roughness of the surfaces. Macroscopic solid surfaces are usually rough and tough only at some points, and capillary condensation takes place only at these contact points. This condensation of liquids, especially water, from vapor can also have additional effects on the physical state of the contact region. If the surfaces contain ions, they will diffuse within the liquid bridge and consequently, changing the chemical composition of the contact zone, and further impacting on the adhesion.

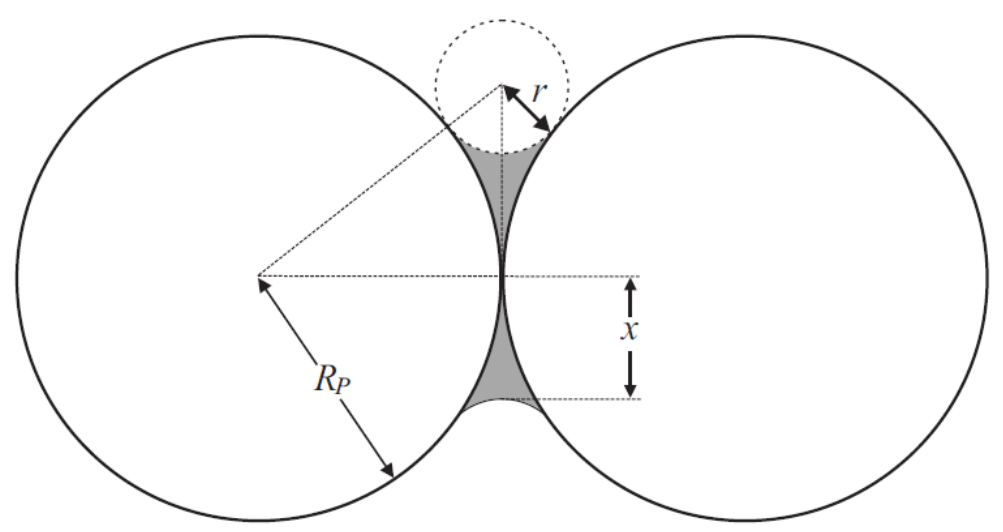

Figure 2.5: Two spherical particles in contact with liquid meniscus.

\subsection{Contact Angle}

Wetting phenomena can be observed every day around us. The behavior of a limited amount of liquid on a solid surface is taken as an indication of the wetting of the surface. If a drop of a liquid is placed on a solid surface, the liquid either spreads completely on the surface or spreads to a finite extent but remains as a discrete drop on the surface. The 
quantitative measure of the wetting is to determine the contact angle, $\theta$, which can be considered as a characteristic of the surface.

Surface and interfacial tensions dominate how a drop of liquid deforms when it adheres to a surface. To derive the relationship between the contact angle and the surface tensions, we consider a typical example where a circular drop on a planar solid surface spreads an infinitesimal amount (Figure 2.6a). While spreading, the radius of the contact zone increases from $a$ to $a^{\prime}=a+d a$. Assuming constant volume, its height decreases from $h$ to $h^{\prime}=h+d h$. In equilibrium, which is the energetically most favorable situation, the change of the Gibbs free energy $d G$ is zero.

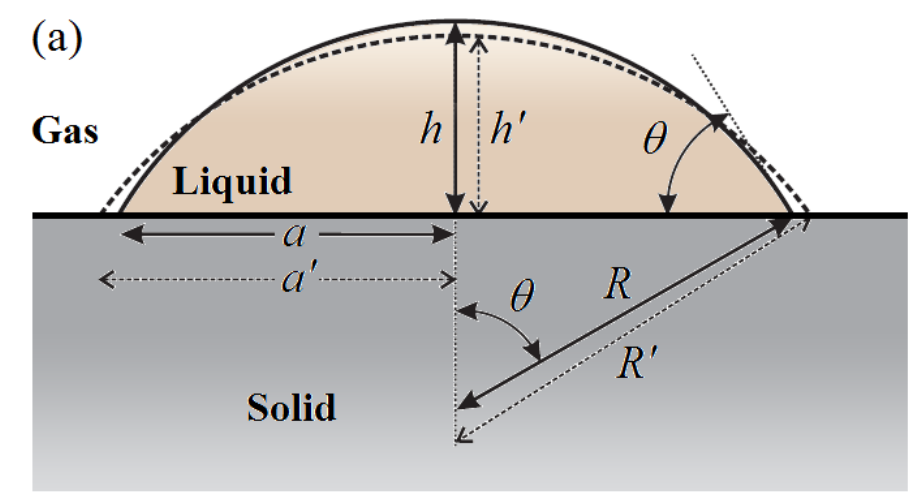

(b)

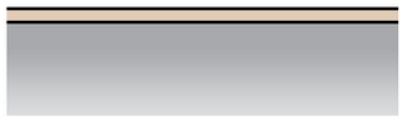

Complete wetting (c)

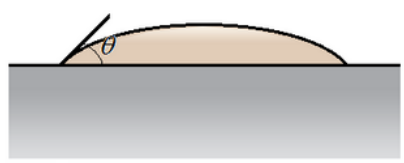

Partial wetting (d)

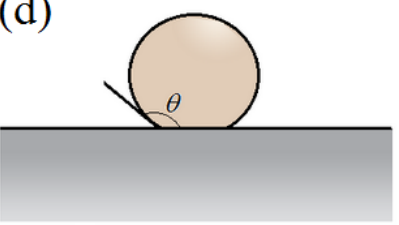

Nonwetting

Figure 2.6: (a) Liquid drop with circular contact area on a planar solid surface spreads an infinitesimal amount. (b-d) The various degrees of wetting: complete wetting $\left(\theta=0^{\circ}\right)$, partial wetting $\left(\theta<90^{\circ}\right)$, and nonwetting $\left(\theta>90^{\circ}\right)$.

On one hand we assume that the drop is so large that a contribution of the Laplace pressure does not lead to a significant change in the Gibbs free energy. On the other hand, 
we assume that the drop is small enough, so that its shape is that of a spherical cap. When the drop spreads, the free solid surface is changed to a solid-liquid interface. The change in area $d A_{S L}=2 \pi a \cdot d a$ leads to a change in surface energy $\left(\gamma_{S L}-\gamma_{S}\right) \cdot d A_{S L}$. In addition, the surface area of the liquid-gas interface changes. The surface of a spherical cap is $A_{L}=\pi\left(a^{2}+h^{2}\right)$. A small change in the contact radius $a$ leads to a change in the liquid surface area of:

$$
d A_{L}=\frac{\partial A_{L}}{\partial a} d a+\frac{\partial A_{L}}{\partial h} d h=2 \pi a \cdot d a+2 \pi h \cdot d h
$$

Here the two variables $a$ and $h$ are not independent. The volume of a spherical cap is $V=\frac{\pi}{6}\left(3 a^{2} h+h^{3}\right)$. Since the volume of the drop is constant we have:

$$
\begin{gathered}
d V=\frac{\partial V}{\partial a} d a+\frac{\partial V}{\partial h} d h=\pi a h \cdot d a+\frac{\pi}{2}\left(a^{2}+h^{2}\right) \cdot d h=0 \\
\frac{d h}{d a}=-\frac{2 a h}{a^{2}+h^{2}}
\end{gathered}
$$

We can use Pythagoras’ law:

$$
\begin{gathered}
(R-h)^{2}+a^{2}=R^{2} \\
a^{2}=2 R h-h^{2}
\end{gathered}
$$

Therefore we get:

$$
\frac{d h}{d a}=-\frac{2 a h}{2 R h-h^{2}+h^{2}}=-\frac{a}{R}
$$

Inserting Eq. (2.4.6) into Eq. (2.4.1) leads to:

$$
d A_{L G}=2 \pi a \cdot d a-2 \pi h \frac{a}{R} \cdot d a=2 \pi a \frac{R-h}{R} \cdot d a=2 \pi a \cdot \cos \theta \cdot d a
$$


The total change in the Gibbs free energy is:

$$
d G=\left(\gamma_{S L}-\gamma_{S}\right) \cdot d A_{S L}+\gamma_{L} \cdot d A_{L G}=2 \pi a\left(\gamma_{S L}-\gamma_{S}\right) d a+2 \pi a \gamma_{L} d a \cdot \cos \theta
$$

At equilibrium $d G / d a=0$ which leads to:

$$
\gamma_{L} \cdot \cos \theta=\gamma_{S}-\gamma_{S L}
$$

Eq. (2.4.9) is known as the Young's equation derived for a quantitative description of wetting phenomena ${ }^{55}$. According to this equation, if the interfacial tension of the bare solid surface is higher than that of the solid-liquid interface $\left(\gamma_{S}>\gamma_{S L}\right)$, $\cos \theta$ has to be positive and the contact angle is smaller than $90^{\circ}$ (partial wetting, Figure 2.6c). If the solid-liquid interface is energetically less favorable than the bare solid surface $\left(\gamma_{S}<\gamma_{S L}\right)$, the contact angle will exceed $90^{\circ}$ because $\cos \theta$ has to be negative (nonwetting, Figure 2.6d). If the system is in equilibrium and we have $\gamma_{S}=\gamma_{S L}+\gamma_{L}$, complete wetting of the surface will occur (Figure 2.6b).

It is found that on real surfaces contact angle measurements often exhibit hysteresis ${ }^{56}$. If we measure the contact angle while the volume of the drop is increasing, we get the socalled advancing contact angle $\theta_{a d v}$. If we afterwards decrease the volume of the drop and determine the contact angle, we measure the so-called receding contact angle $\theta_{\text {rec }}$. Usually $\theta_{a d v}$ is significantly higher than $\theta_{r e c}$. The difference $\theta_{a d v}-\theta_{r e c}$ is called contact angle hysteresis. Possible causes of hysteresis are roughness, inhomogeneity, line tension, energy dissipation etc. 


\subsection{Hydrophobic/Hydrophilic Interactions}

All molecules including polar and non-polar molecules attract each other at distance of the order of a few Angstrom ( $\AA$ ) by weak attractive force. It is known that water molecules can form “strong” intermolecular interactions termed hydrogen bonds. The strength of hydrogen bond is always between van der Waals force and covalent bonds. The polarity and hydrogen-bond strength of water make it a unique solvent and highly active molecule. When molecules are dissolved in water, polarity and hydrogen bonds are playing important roles in the formation of interactions between solutes and water molecules.

“Hydrophobic” means “water-hating (or fearing)”, and it always refers to the category of substance which is immiscible with water. If the contact angle is above $90^{\circ}$, the surface can be called hydrophobic. Between hydrophobic surfaces a completely different interaction is observed. Hydrophobic surfaces strongly attract each other when they are immersed in water. This attraction is called hydrophobic interaction. This interaction summarizes all of forces which reduce the solubility of non-polar molecules in water. The first direct evidence that the interaction between solid hydrophobic surfaces exceeds the van der Waals attraction at large separations by more than one order of magnitude was

provided by Israelachvili and Pashley ${ }^{57}$. The exact origin of hydrophobic interaction is, however, still under debate. On the molecular level, the hydrophobic force between two molecules is complex; it involves many other molecules and is of much longer range than a typical covalent bond. One hypothesis is that the interaction arises from the configurational rearrangement of water molecules as two hydrophobic species come 
together. The restructuring of water molecules is entropically unfavorable; in other words, rather than forming new structure with non-polar solutes, water prefers maintaining the existing interactions with same water molecules. Studies of hydrophobic interaction are of importance because this interaction plays a major role in many surface phenomena, in molecular self-assembly, in biological membrane structure and in the formation of the compact structure of proteins.

In contrast, "hydrophilic” means "water-loving”, and hydrophilic groups prefer to be in contact with water rather than with each other. Hydrophilic surface should have contact angles close to zero. Hydrophilic interaction is the summation of all intermolecular interactions (including electrostatic) that cause the process of hydration (or salvation, the clustering process of water molecules around a solute particle). It is clear that polar molecules are easy to dissolve in water, because it requires low energy to break the hydrogen bonds already established among water molecules and to induce new hydrogen bonds which are participated by polar solute molecules. In addition, some uncharged and even non-polar molecules can be hydrophilic if they have the right geometry and/or they contain electronegative atoms capable of associating with the hydrogen-bond network in water. 


\section{Chapter 3. Design of Shear-Force/Acoustic Near-Field Microscopy}

\subsection{The Quartz Tuning Fork}

Precision tip-sample distance control is a need in many SPM techniques. Several different approaches exist to achieve the feedback control that maintains the probe at constant height above the sample with atomic-level accuracy. For example, a tunneling current flowing between tip and sample is used as the signal in feedback loop of the STM, whereas in feedback control of the AFM the tunneling current is replaced by tip-sample forces detected by cantilever based force sensors.

Aside from the choice of silicon cantilevers, quartz tuning forks (TFs) have been widely used as force sensors in the short-range noncontact mechanical force distance control of SPMs. The miniaturized quartz TF consisting of two prongs connected at one end is a commercially produced piezoelectric oscillator whose resonance frequency is defined by the properties of the material from which it is made and by its geometry. Quartz TFs are primarily designed as frequency standards in clocks and watches. However, over the past decade they have found application in scanning probe instruments, particularly in NSOM, monitoring the separation between probe and surface ${ }^{35}$. It was subsequently demonstrated that TFs could be used as both sensor and actuator to measure topographical features on the nanoscale ${ }^{58,59}$. Quartz TFs are cheap, robust, and easy to install and to use. They operate at a higher frequency than most other vibrating sensors, and they are highly sensitive indicators of the physical properties of the medium in where they are immersed. Quartz TFs offer important advantages as a sensor platform. High 
quality factor allows high sensitivities at small oscillation amplitudes. Low energy dissipation assures low temperature compatibility. In comparison with conventional microfabricated cantilevers, high spring constant of TFs enables stable operation without the tip jumping to contact at very small tip-sample distances. Quartz TFs are highly insensitive to magnetic fields, thus they become useful as a field-independent senor in measurements as a function of magnetic field. Moreover, only two wires are needed to drive and readout a TF sensor, therefore the mechanical design and external electronics are minimized making easy the implementation of TFs in SPMs under UHV condition. Because of these convenient features the TF has become an indispensable component for the design of a SPM.

The unique properties of TFs are based on the native piezoelectric effect of quartz crystals. Piezoelectricity defines the ability of a material to convert a voltage to a mechanical displacement, and conversely, to generate electrical charges by the deformation of the crystalline matrix. To convert a TF into an SPM force sensor, a sharpened tip is attached along one of its prongs so that the tip protrudes out of the end of the prong ${ }^{35}$. The TF is used as a force sensor, while the tip acts as a shear-force pick-up. To be a compact sensor, the TF is driven to oscillate in such a way that the tip vibrates parallel to the sample surface. Both prongs of the TF are piezoelectrically coupled through the metallic contact pads. The geometry of the contacts and the coupling between the two prongs insure that only one resonant vibration mode of the TF is excited. This in turn yields an electric current proportional to the deformation of the TF prongs. When implemented for tip-sample distance control, the TF is oscillating at its resonance 
frequency. As the tip approaches normally to and is within tens of nanometers of the sample, a reduction of the piezoelectric current is measured due to the presence of shear forces originating from the tip-sample interaction. For topography imaging purpose, this current signal is used in a feedback loop to maintain constant tip-sample distance.

\subsubsection{Mechanical and Electrical Properties of Quartz Tuning Forks}

The behavior of TF sensors is modeled in a standard way as a damped harmonic oscillator $^{35,60}$ (Figure 3.1a) and electrically, as a series resistance-inductor-capacitor (RLC) circuit in parallel with package capacitance by the Butterworth-Van Dyke model $^{60,61}$ (Figure 3.1b). The RLC circuit provides a convenient electrical analogue to the mechanical properties of the TF: the resistance represents the acoustic losses in the material and its environment, the inductor represents the mass of the resonators, and the capacitor represents the stiffness of the equivalent spring.

(a)

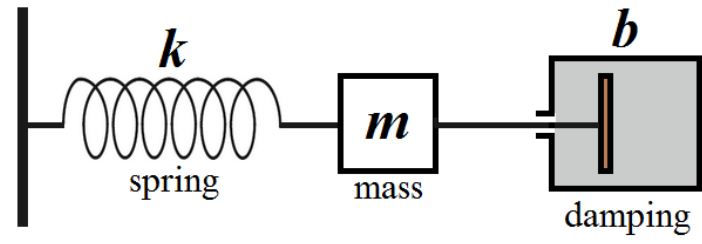

(b)

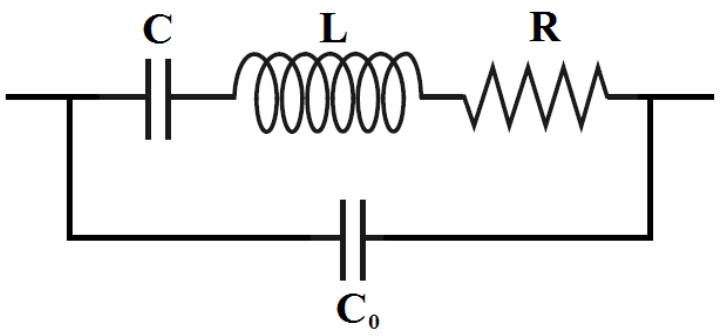

(c)
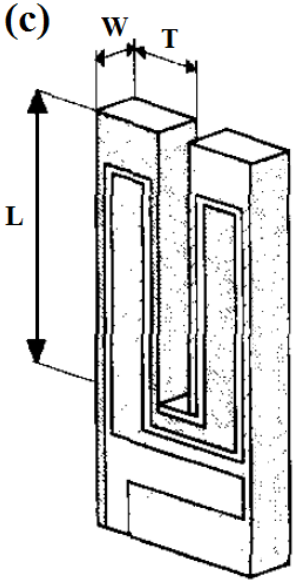

Figure 3.1: (a) Mechanical model of the TF resonator as a damped harmonic oscillator. (b) Equivalent electrical model of the TF oscillator as a series RLC circuit. (c) Sketch of the quartz TF drawn by Karrai and Grober ${ }^{35}$. 
At sufficiently small oscillation amplitude $x(t)$, the TF can be described as a simple harmonic oscillator subject to a driving force $F_{0} \cos (\omega t)$ and a damping force with linear dependence on velocity. The equation of motion is expressed by:

$$
m \ddot{x}(t)+b \cdot(t t)+k x(t)=F_{0} \cos (\omega t)
$$

where $m$ is the effective mass of the TF, $b$ is the damping coefficient, $k$ is the spring constant of the equivalent spring. The effective mass and the damping coefficient depend on the medium around the oscillating TF. The solution of Eq. (3.1.1) can be written as:

$$
x(t)=A(\omega) \cos (\omega t+\varphi)
$$

where $A(\omega)$ is the amplitude and $\varphi$ is the phase difference with respect to the external driving force. It is convenient to introduce the angular resonance frequency of the oscillating system, $\omega_{0}=\sqrt{\frac{k}{m}}$, thus the amplitude and the phase are given by:

$$
\begin{gathered}
A(\omega)=\frac{F_{0}}{\sqrt{b^{2} \omega^{2}+m^{2}\left(\omega_{0}^{2}-\omega^{2}\right)^{2}}} \\
\tan \varphi=-\frac{b \omega}{m\left(\omega_{0}^{2}-\omega^{2}\right)}
\end{gathered}
$$

The geometry of the TF in Figure 3.1c is characterized by the length $L$, width $W$, and thickness $T$ of a prong. The theoretical spring constant can be calculated from the formal ${ }^{35}$ :

$$
k=\frac{E}{4} W\left(\frac{T}{L}\right)^{3}
$$


where $E=7.87 \times 10^{10} \mathrm{~N} / \mathrm{m}^{2}$ is the Young modulus of quartz. The effective mass of one prong in vacuum is:

$$
m_{v a c}=0.24267 \rho_{q} L W T
$$

where $\rho_{q}=2659 \mathrm{~kg} / \mathrm{m}^{3}$ is the density of quartz.

Equivalently, the motion of TF oscillator can also be modeled by a similar electrical differential equation. The TF is excited with AC voltage $U_{0} \cos (\omega t)$ while the frequency is slowly swept through resonance. The signal received from the TF is a current $I(t)$ owing to the piezoelectric effect. The stresses due to the TF deformation induce charges $Q(t)$ and thus the current is proportional to the derivative of the TF mechanical deflection $^{62,63}$ :

$$
Q(t)=2 \alpha \cdot x(t) \quad \text { or } \quad I(t)=2 \alpha \cdot \frac{d x(t)}{d t}
$$

where $\alpha$ is the piezo-electromechanical coupling constant. The exact value depends on the specific type of TFs. The corresponding Butterworth-Van Dyke equation for the current is:

$$
L \ddot{I}(t)+R \dot{I}(t)+\frac{1}{C} I(t)=U_{0} \cos (\omega t)
$$

where $L$ is inductance, $R$ is resistance, and $C$ is capacitance. In addition, at resonance equating the electrical energy stored on the TF capacitance to the dissipated energy of two prongs of the TF leads to:

$$
\alpha=\sqrt{\frac{k C}{2}}
$$


Comparing Eq. (3.1.8) and Eq. (3.1.1) obtains:

$$
R=\frac{b}{2 \alpha^{2}}, L=\frac{m}{2 \alpha^{2}}, C=\frac{2 \alpha^{2}}{k}, \text { and } \omega_{0}=\sqrt{\frac{1}{L C}}
$$

The electrical model of the TF differs from the classical damped oscillator by the presence of a package capacitance $C_{0}$ parallel to the RLC series components. The package capacitance is mainly determined by the geometrical arrangement of the contacts on the crystal, the dielectric properties of the quartz and by cable capacitances ${ }^{62}$. The result of this capacitance is an antiresonance characterized by a minimum in the current at a frequency above the resonance frequency. In Figure 3.2a both the resonance and antiresonance are clearly seen in the experimental transfer function in which the current through the TF vs frequency is measured.
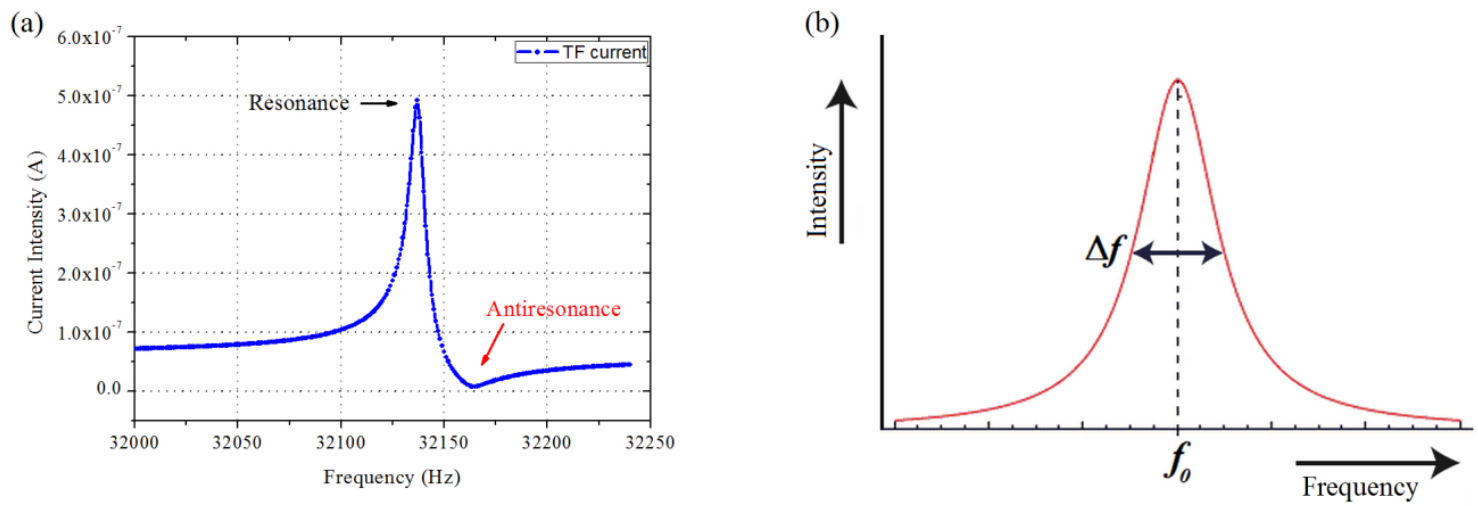

Figure 3.2: (a) Current measurement of an opened TF as a function of frequency. The resonance is followed by the antiresonance. (b) Definition of the quality factor based the resonance curve. $\Delta f$ is the FWHM of the waveform.

Here we define the so-called quality factor of the TF, $Q$. On one hand, Figure $3.2 \mathrm{~b}$ demonstrates the qualitative measure of the quality factor based on the frequency response of the TF. By definition, the quality factor is given by ${ }^{35}$ : 


$$
Q=\frac{f_{0}}{\Delta f}
$$

where $f_{0}$ is the resonance frequency, and $\Delta f$ is the full width at half maximum (FWHM) of the Lorenzian-shaped waveform. On the other hand, the quality factor refers to the ratio of the energy stored in the resonator to the energy dissipated during each oscillation period. Since the damping coefficient is related to the $\mathrm{FWHM}^{35}$, we have:

$$
Q=\frac{\sqrt{m k}}{b}=\frac{1}{R} \sqrt{\frac{L}{C}}
$$

From the fundamental definition, the quality factor can either be increased by injecting energy into the TF, or be decreased by removing energy during each cycle. A typical value of the quality factor of a TF is in the order of $10^{3} \sim 10^{4}$ at ambient conditions with the resonance at $2^{15}=32768 \mathrm{~Hz}$. Such a high quality factor originates from the fact that there is no center-of-mass motion.

\subsubsection{Mechanically-Driven and Electrically-Driven Modes of Quartz Tuning Forks}

In operation, the TF can be excited in two different modes. One method referred to the mechanically driven (MD) mode is that the TF is externally vibrated near its resonance frequency by a driving piezoelectric element which is attached to the base of the TF. The other method referred to the electrically driven (ED) mode is that the TF is excited by applying an AC voltage across its terminals to take full advantage of the selfactuating and self-sensing properties. Thus, neither external actuator nor detector is required to actuate it or to sense its motion while driving the TF electrically. In ED mode, however, the measured electric signal cannot fully reflect the true mechanical motion of 
the TF. The reason is that the measurement of the total induced current contains two contributions $^{62}: I_{m}$ is the current generated by the mechanical vibration of the TF prongs due to the piezoelectric effect of the quartz, and $I_{c}$ is the capacitive current through the TF. In contrast to symmetric waveform in the MD mode, the resonance curve of the ED mode operation is highly asymmetric.

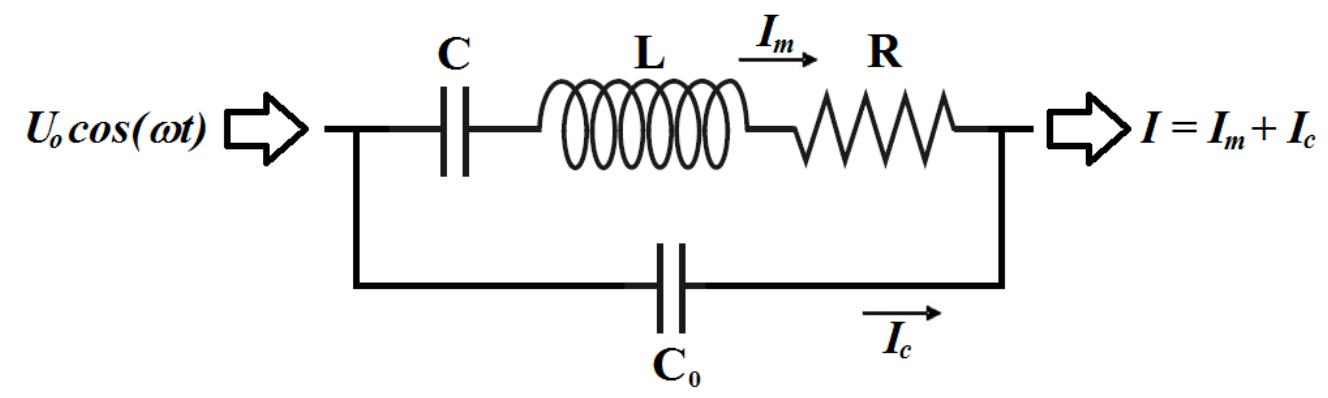

Figure 3.3: The equivalent circuit of the TF in electrical driving mode.

To overcome problems caused by the nonlinear feature of TFs, a simple way enables conversion between the ED and MD operation modes. As shown in Figure 3.3, the measured electric current is written as ${ }^{64}$ :

$$
I(t)=A \sin (\omega t+\varphi)=I_{m}(t)+I_{c}(t)
$$

where the amplitude $A$ and the phase $\varphi$ are given by:

$$
\begin{gathered}
A=\frac{I_{0} \omega}{Q \omega_{0}} \sqrt{\frac{1+2\left(\frac{C_{0}}{C}\right)\left(1-\frac{\omega^{2}}{\omega_{0}^{2}}\right)+\left(\frac{C_{0}}{C}\right)^{2}\left[\left(1-\frac{\omega^{2}}{\omega_{0}^{2}}\right)^{2}+\left(\frac{\omega}{Q \omega_{0}}\right)^{2}\right]}{\left(1-\frac{\omega^{2}}{\omega_{0}^{2}}\right)^{2}+\left(\frac{\omega}{Q \omega_{0}}\right)^{2}}} \\
\varphi=\arg \left[\frac{\omega}{Q \omega_{0}}+i\left\{1-\frac{\omega^{2}}{\omega_{0}^{2}}+\left(\frac{C_{0}}{C}\right)\left[\left(1-\frac{\omega^{2}}{\omega_{0}^{2}}\right)^{2}+\left(\frac{\omega}{Q \omega_{0}}\right)^{2}\right]\right\}\right]
\end{gathered}
$$


with $I_{0}=U_{0} / R$, and $-\pi \leq \varphi \leq \pi$. We assume the capacitive current can be expressed by $^{64}$ :

$$
I_{c}(t)=\omega C_{0} \cdot U_{0} \sin (\omega t)
$$

Therefore, the induced current due to the harmonic oscillation can be calculated through ${ }^{64}$ :

$$
I_{m}=I(t)-I_{c}(t)=A \sin (\omega t+\varphi)-\omega C_{0} \cdot U_{0} \sin (\omega t)=A_{m} \sin \left(\omega t+\varphi_{m}\right)
$$

and the amplitude and the phase are given by:

$$
\begin{aligned}
A_{m} & =\sqrt{A^{2}-2 \omega C_{0} U_{0} \cdot A \sin \varphi+\left(\omega C_{0} U_{0}\right)^{2}} \\
\varphi_{m} & =\arg \left[A \cos \varphi+i\left(A \sin \varphi-\omega C_{0} U_{0}\right)\right]
\end{aligned}
$$

Eq. (3.1.18) and Eq. (3.1.19) can then be used to convert the motion of the ED mode TF, characterized by $A$ and $\varphi$, to that of the MD one, described by $A_{m}$ and $\varphi_{m}$.

Figure 3.4a demonstrates the comparison between the measured frequency response of a TF in electrical driving mode and the calculated resonance curve using Eq. (3.1.14) and Eq. (3.1.15). They exhibit excellent agreement with each other. Figure 3.4b presents the resulting motion of the same TF after removing the effect of package capacitance. Using Eq. (3.1.18) and Eq. (3.1.19), the converted curve shows high symmetry. 
(a)

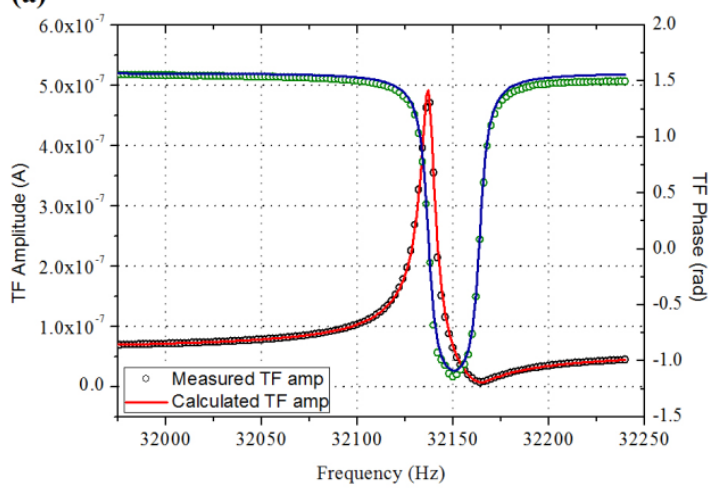

(b)

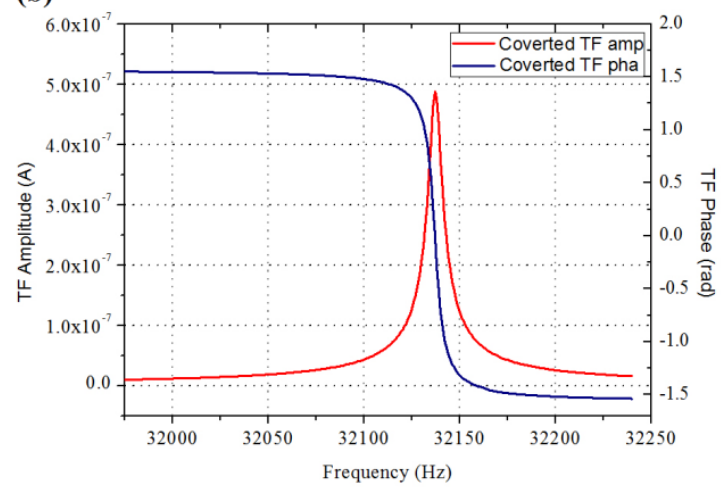

Figure 3.4: (a) Comparison of the measured (open circle) and calculated (solid line) amplitude and phase of a TF in electrical driving mode. (b) The resulting converted curve reflecting the true harmonic motion of the same TF.

\subsection{Probe Fabrication}

\subsubsection{Fabrication of Gold Tips}

Sharpened metallic tips are commonly used to probe the sample properties in various SPM techniques. Therefore, different methods have been developed for fabrication of tips. Among these methods, mechanical cutting is apparently the easiest way, but the reproducibility of this method is poor. Field-ion technique is used for preparation of welldefined single-atom tips, but this is an expensive and complicated method not affordable for most researchers. The most practical approach to fabricate metallic sharp tips is electrochemical etching.

The basic principle in electrochemical etching of a metal wire is to dip a small diameter metal wire into an electrolyte solution in which a counter electrode is sitting and to apply a AC or DC voltage between these two electrodes until enough dissolution of the wire has happened so that is displays a sharp tapered shape. The choice of the electrolyte 
and of the voltage applied depends on the material used to make tips. Tungsten tips, which are widely used in SPM, can be easily acquired via electrochemical etching ${ }^{8}$. For SPM studies in air, however, a drawback of tungsten tips is that tungsten will slowly oxidize in air. In comparison with tungsten, gold is an inert metal so that it is nearly impossible to form oxide layers outside gold. Thus, it is favorable to choose gold as the material of SPM probe while performing SPM experiments in air.

Gold is a noble metal that is unaffected by air, moisture and most corrosive reagents. $\mathrm{Au}(\mathrm{I})$ and $\mathrm{Au}$ (III) are two common oxidation states of gold in its compounds. Applying an appropriate voltage, gold can be dissolved in solutions containing $\mathrm{CN}^{-}$of $\mathrm{Cl}^{-}$ions. However, the former is very toxic and requires a high voltage $(\geq 10 \mathrm{~V})$. The advantage of the hydrochloric $(\mathrm{HCl})$ acid etching method is its nontoxicity and low etching voltage.

Herein, we demonstrate an easy and low cost method based on electrochemical etching for fabrication of gold tips. Our gold tips were made from either a 0.003 inches (76 $\mu \mathrm{m}$ ) diameter wire or a 0.004 inches $(100 \mu \mathrm{m})$ diameter wire (Au, purity 99.95\%, ESPI). As shown in Figure 3.5, the etching setup is similar to the one used for etching tungsten wires. Gold wires to be etched are cut into pieces $\sim 1 \mathrm{~cm}$ long, acting as the anode electrode. A small ring of $\sim 3 \mathrm{~mm}$ diameter made from a 0.008 inches ( $200 \mu \mathrm{m}$ ) gold wire (Au, purity 99.95\%, ESPI) is used as the cathode electrode. The wire holder fixed to a Z-direction translator allows precise adjustment of the length of the wire immersed in the solution. A home-made DC power supply provides the bias voltage necessary to generate the etching reactions---in our case, we attempt $5.0 \mathrm{~V}_{\mathrm{DC}}$. An electronic microcontroller is inserted in the circuit to monitor the current between the 
anode and the cathode. A home-made controlling switch is added as an option to quickly cut off the current when the etching process is completed. In electrochemical etching, the cathode ring held a drop of concentrated $\mathrm{HCl}$ acid by surface tension. The gold wire threaded through it and was vertically dipped into the solution. Etching of the gold wire started when the voltage was applied, and ended up with the formation of a sharp tapered tip. After etching the tip was taken off from the station and rinsed with distilled water to remove any residual $\mathrm{HCl}$.
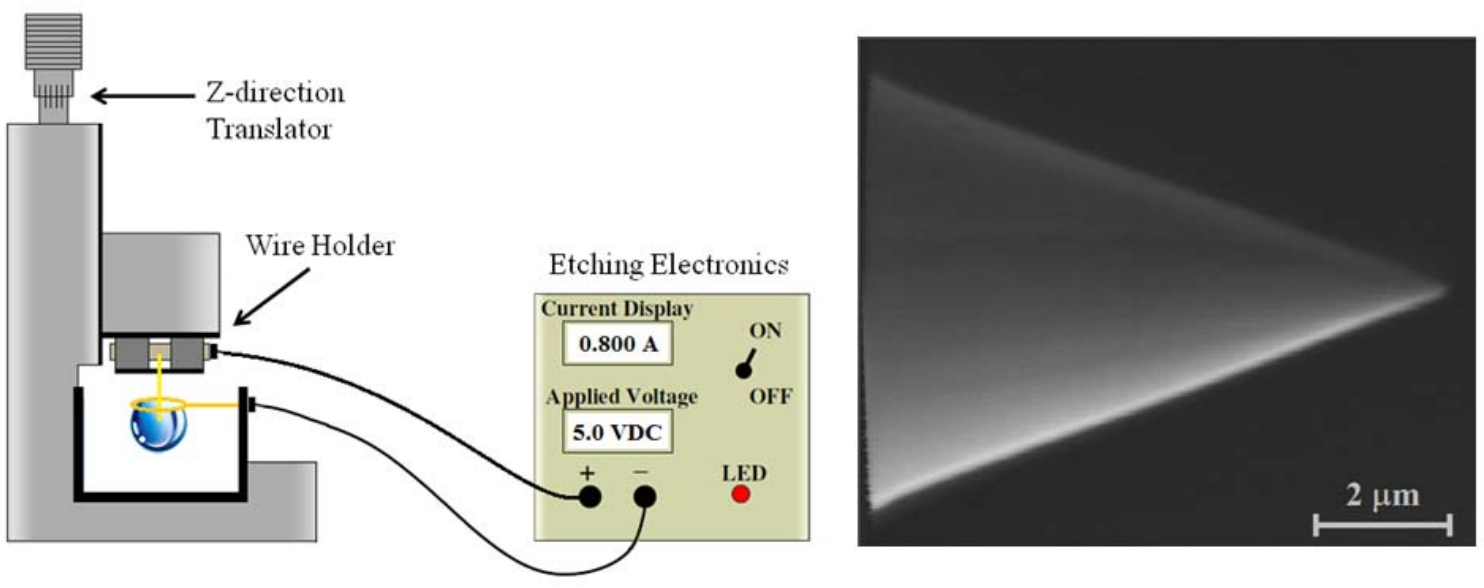

Figure 3.5: Sketch of the electrochemical etching station and the scanning electron microscope (SEM) image of the gold tip using DC etching method.

The overall ionic reactions dominating this electrochemical etching of gold in a concentrated $\mathrm{HCl}$ solution are ${ }^{65}$ :

$$
\begin{array}{ll}
\mathrm{Au}+4 \mathrm{Cl}^{-} \longrightarrow \mathrm{AuCl}_{4}^{-}+3 \mathrm{e}^{-}, & \mathrm{E}_{0}=1.002 \mathrm{~V} \\
\mathrm{Au}+2 \mathrm{Cl}^{-} \longrightarrow \mathrm{AuCl}_{2}^{-}+\mathrm{e}^{-}, & \mathrm{E}_{0}=1.154 \mathrm{~V} \\
\mathrm{AuCl}_{2}^{-}+2 \mathrm{Cl}^{-} \longrightarrow \mathrm{AuCl}_{4}^{-}+2 \mathrm{e}^{-}, & \mathrm{E}_{0}=0.926 \mathrm{~V}
\end{array}
$$


Similarly to the etching process of tungsten wires, when the gold wire is immersed into the electrolyte, capillary forces yield the formation of a meniscus of solution around the tip at the air-liquid interface. At the beginning of the etching procedure, the diameter of the wire and thus the etching current are at a maximum. With ongoing time, the diameter of the wire and the current decrease, as well as the meniscus decreases in height. Generally, etching proceeds more rapidly at the meniscus. Thus, a necking-in occurs within the meniscus region. At some point, the etched part of the wire becomes so thin its tensile strength cannot sustain the lower portion of the wire, the latter drops off and a sharp tip is left behind. Once the lower part falls down, the etching current decreases abruptly, and the entire process is self-terminating.

In contrast to the etching process of tungsten wires, at a high rate of electrochemical etching, ionic reactions mentioned above consume a large amount of $\mathrm{Cl}^{-}$ions near the interface, leading to a depletion of $\mathrm{Cl}^{-}$ions in this region. Subsequently, a layer of gold oxides forms, which passivates the gold surface; as a result, the etching current significantly decreases. However, the fast resupply of $\mathrm{Cl}^{-}$ions from the bulk solution can facilitate the dissolution of gold oxides as $\mathrm{AuCl}_{4}{ }^{-}$. As a consequence, the bare gold surface re-exposes to the $\mathrm{HCl}$ solution and, the etching current increases. This process described above occurs again and again, establishing a current oscillation ${ }^{65}$. Evidence for this is the periodic change of the etching current during the etching procedure shown in Figure 3.6. 

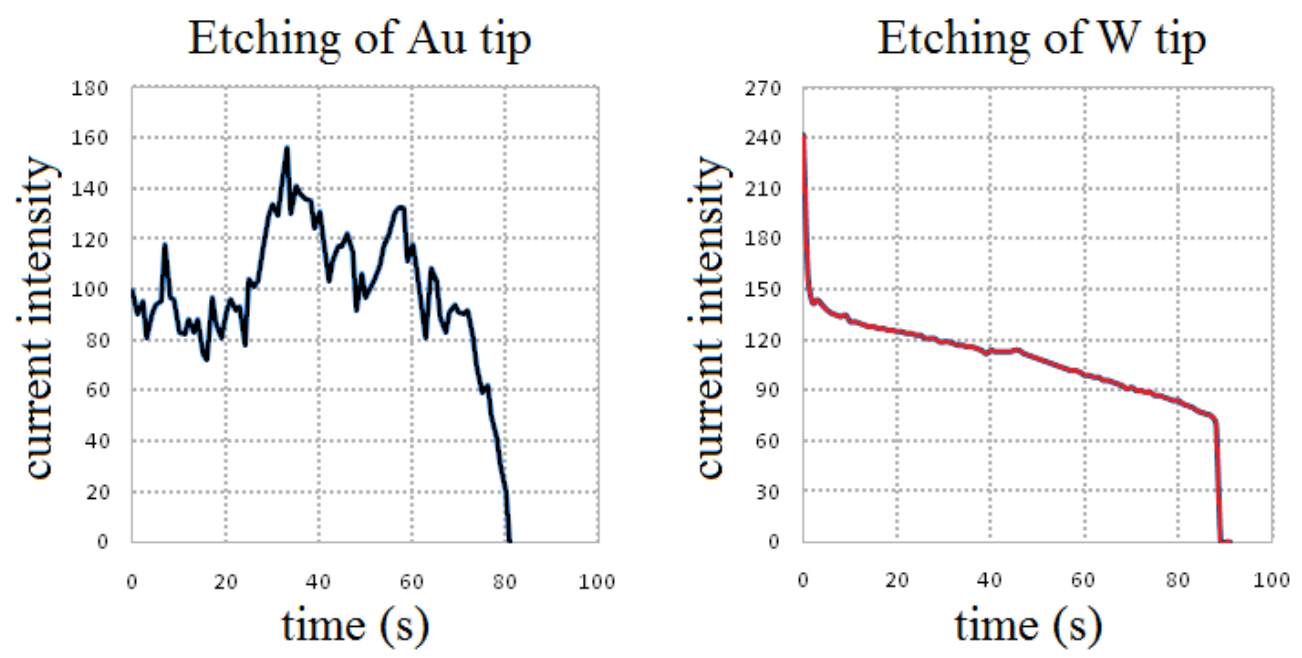

Figure 3.6: The etching current intensity monitored during the etching procedure of gold wire (left), and the etching process of tungsten wire (right).

The gold ring used as cathode is etched during the etching process. However, since porous deposits were cover the ring surface causing an electrode of somewhat lower conductivity, the ring has to be cleaned or replaced periodically. In addition, side reactions including the evolution of $\mathrm{Cl}_{2}$ and $\mathrm{O}_{2}$ cause a severe bubbling effect. The bubbling effect could be a problem in etching processes, because it may bring an etching interruption and a rough structure of tip surface. Thus, it should be reduced as much as possible. Considering the role of ethanol in silicon industry, where ethanol is used to reduce the bubbling and, thereby to produce a smooth tip surface, a certain amount of ethanol was added to the $\mathrm{HCl}$ solution to reduce the bubbling effect with the aim to obtain smooth gold surfaces. The optimized electrolyte mixture is composed of concentrated $\mathrm{HCl}$ acid and ethanol with the proportion 1:1 in volume.

Several factors can impact the etching process, resulting in sharpened tips with differences in morphology. These parameters include the applied voltage, the immersion 
depth of gold wires into solution, and the ratio of concentrated $\mathrm{HCl}$ acid to ethanol in the electrolyte. Experimental results showed that the etching current was influenced by the immersion depth of gold wires. It was easier to obtain tips with sharper apexes when the etching current was relatively high. For the purpose of using gold tips as a probe in SPM studies, the optimized immersion depth of gold wires could be: $0.15 \mathrm{~mm}$ for $76 \mu \mathrm{m}$ diameter gold wires, and $0.40 \mathrm{~mm}$ for $100 \mu \mathrm{m}$-diameter gold wires. Different shapes of tapered gold tips can also be obtained by varying other parameters depending on the specific requirements in experiments.

Experiments demonstrate that gold tips can be fabricated through DC etching. However, the tip surfaces are relatively rough. Alternatively, AC etching may be a favorable way for fabrication of gold tips. The electric circuit used in AC etching procedure consists of a laboratory Variac used as the primary source (115 V), and a step down transformer (Figure 3.7). The Variac controls the input voltage into the step down transformer, so that the voltage can be brought down to the range reported in literatures of AC etching method. The main procedure of AC etching is as same as that of DC etching described above. The same electrolyte of a mixture of concentrated $\mathrm{HCl}$ acid and ethanol was chosen. A voltage of $1.8 \mathrm{~V}_{\mathrm{rms}}$ or around was applied to trigger chemical reactions necessary to etch gold wires. There are a few factors that affect the shape of resulting tips. In order to improve the reproducibility of etching experiments, the parameters, such as the applied voltage and the length of the wire dipped into the solution should be well controlled. It was found that AC etching method is an advisable way to reproducibly produce good gold tips with smooth surfaces. 


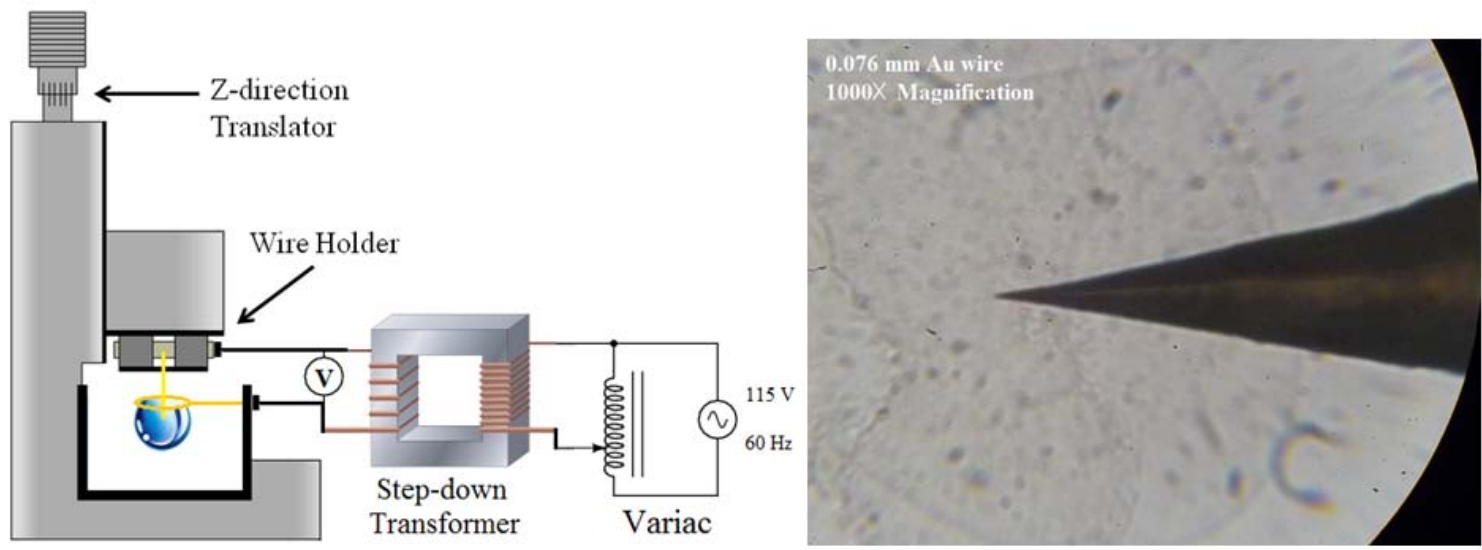

Figure 3.7: Sketch of the electrochemical etching station and the optical image (1000X magnification) of the gold tip using AC etching method.

\subsubsection{Thinning of Optical Fibers}

Aperture probes are an important class of NSOM probes. The first step to produce such probes is to taper optical fibers yielding conical tips. A convenient method is based on chemical etching optical fibers at the meniscus between hydrofluoric (HF) acid and an organic over layer. A sharpened tip is formed due to a decreasing meniscus height as the fiber diameter is reduced by the etchant ${ }^{66}$. However, the goal of our method discussed here is not to create a tapered tip at the apex but to obtain a fiber with reduced diameter and flat end.

In general, the basic structure of an optical fiber contains three parts: the core, the cladding, and the polymer coating (jacket). The central portion where most of the light travels is the core, which is a cylindrical rod made of glass. Surrounding the core there is a layer made of a dielectric material with a lower index of refraction, called the cladding. For extra protection from physical damage, the cladding is enclosed in an additional layer 
termed the coating or jacket. The material used for a coating is generally a type of polymer. To reduce the cladding diameter without forming tapered tip, the HF acid used in conventional etching of glass fiber was replaced by a buffered HF (BHF) solution consisting of a mixture with a volume ratio of $\left[\mathrm{NH}_{4} \mathrm{~F}\right.$ aqueous solution (40 wt.\%)]: [HF acid (50 wt.\%)]: [ $\left.\mathrm{H}_{2} \mathrm{O}\right]=X: 1: 1$, where $X$ denotes a variable volume ${ }^{67}$. We tested two types of optical fibers: FS-SC-6324 and 1310BHP (single mode optical fiber, the cladding diameter $125 \mu \mathrm{m}$, THORLABS Inc.). Prior to chemical etching, optical fibers were cut into pieces of $\sim 5 \mathrm{~cm}$ long. For each piece, the fiber was well cleaved, and its polymer coating was stripped off. Subsequently, these fibers were vertically immersed in BHF for reducing the cladding thickness rather than tapering the core. A thin layer of oil was added to prevent dangerous vapors escaping from the etching container. After an etching time, the thinned fibers were taken off from BHF and rinsed with distilled water for removing any residual chemical. Figure 3.8a shows the images of the same 1310BHP fiber before and after a 2.5-hour thinning procedure. A dramatic decrease in fiber diameter is obtained, without tapering the fiber end. The reason to keep the core end flat is that the dissolution rates of the core and cladding are equal.

There are several concerns with etching procedure. If the ratio of the composition of BHF solution is fixed ( $X$ is fixed), the resulting profile of fiber strongly depends on the concentration of germanosilicate $\left(\mathrm{GeO}_{2}\right)$ doped in the core. Unfortunately, in our case, information regarding the Ge concentration in the core was missing. Therefore, the value of $X$ has to be suitable to maintain the fiber end flat. In our experiments, using the BHF solution with the volume ratio of $\mathrm{NH}_{4} \mathrm{~F}: \mathrm{HF}: \mathrm{H}_{2} \mathrm{O}=2: 1: 1(X=2)$, thinning of optical 
fiber was achieved. In additional, after the fiber to be etched and the recipe of BHF solution being determined, etching time is crucial to obtain fibers with different diameters. As shown in Figure 3.8b, for both two types of glass fibers, the diameter of the resulting fiber monotonously decreases for increasing etching time. Thinning of glass fibers can be controlled through adjusting the time of chemical etching.

(a)

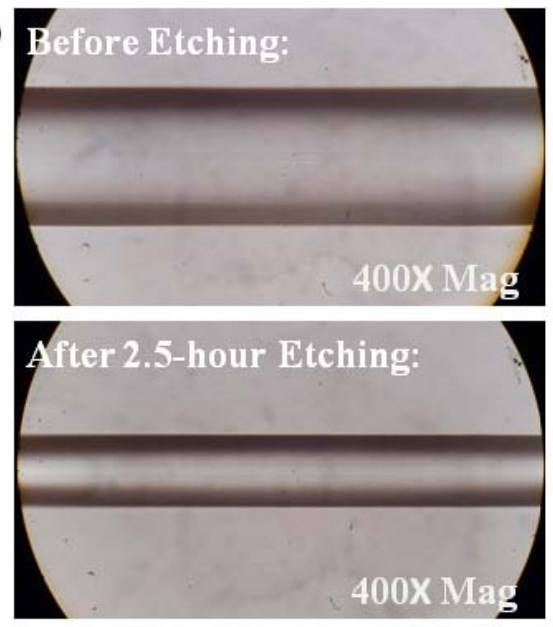

(b)

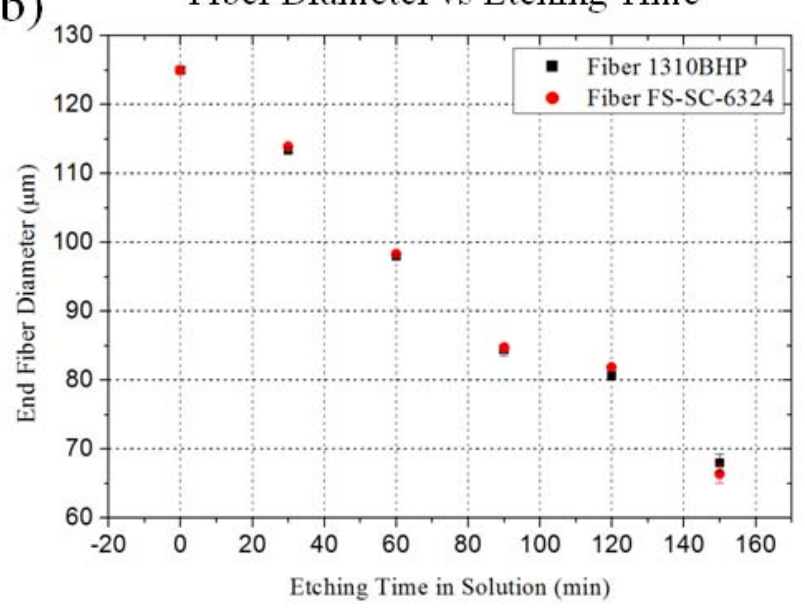

Figure 3.8: (a) Optical images of the same glass fiber before and after a 2.5-hour etching. (b) The plot of fiber diameter vs etching time shows the resulting fiber diameter decreases upon increasing etching time.

\subsection{Microscope Design}

The microscope station shown in Figure 3.9 consists of the following parts:

(1) The base plate (1a) where three high-resolution screws (1c) (AJS100-2, Newport Corp.) are fixed to it. The fine screws are used to roughly control the separation between microscope probe and sample surface. 
(2) The scanner head (1b) where a piezo-actuated linear nanopositioning stage (1d) (Nano-OP65, Mad City Labs Inc.) is fixed to it. The nanopositioner enables fine movement of the probe in vertical (Z-axis) direction with nanometer precision.

(3) The sample stage used to support the top portion.

(4) The X-Y scanner and the TF holder made of plastic and macor.

(5) The acoustic sensor (SE40-Q, Dunegan Engineering Consultants, Inc) held tightly onto the sample stage.

(6) The humidity chamber made of clear acrylic material. 


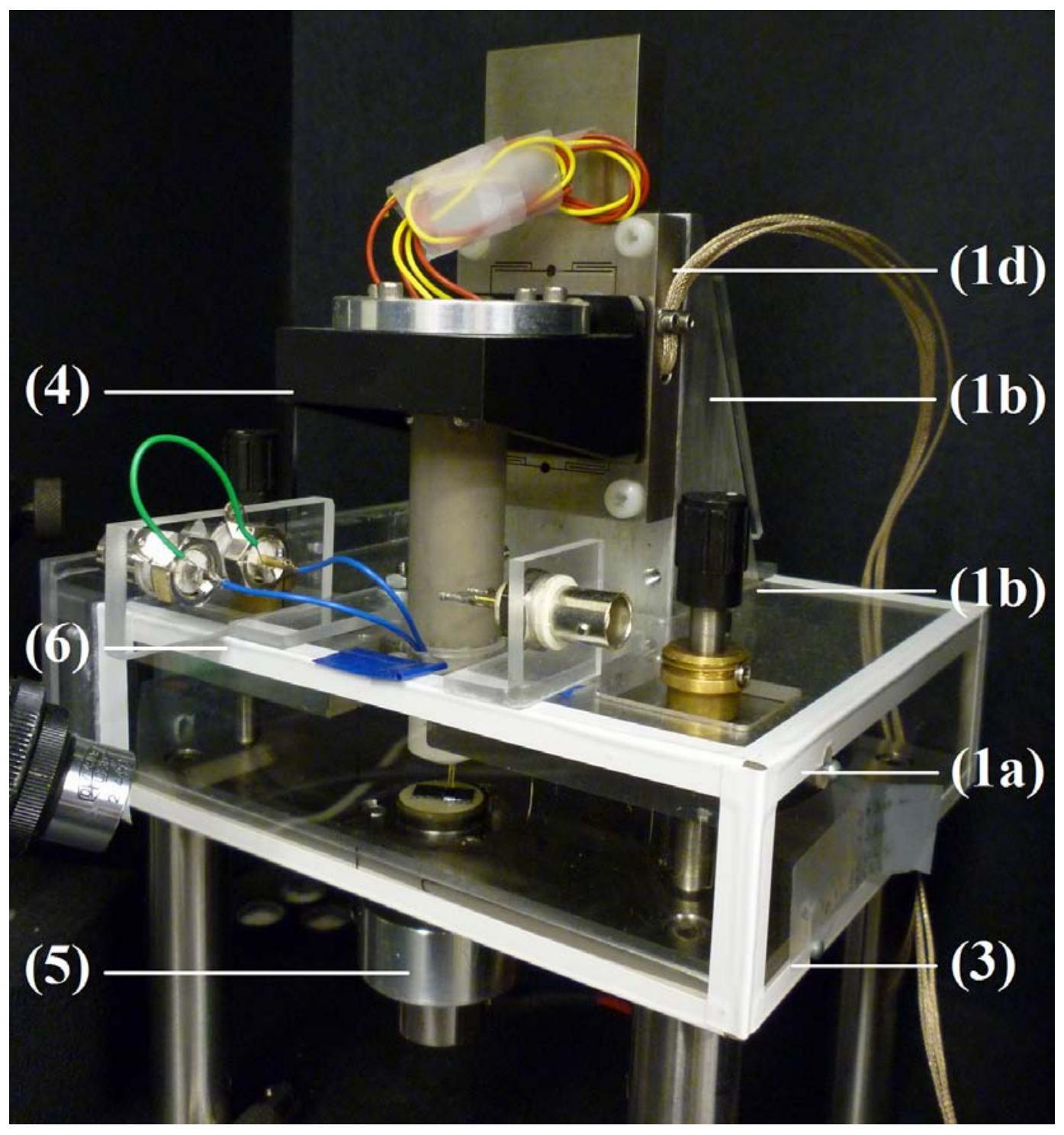

Figure 3.9: The key components of a microscope stage.

\subsection{Detection Electronics}

Experimental data collection is achieved using Stanford Research Systems digital lock-in amplifiers. In a typical experiment, the TF is driven by the sinusoidal voltage from the built-in signal generator of one lock-in SR850 (lock-in \#1). The same lock-in 
amplifier is also used to detect the electric response of the TF. Simultaneously, the acoustic sensor is directly hooked up another lock-in SR850 (lock-in \#2) so as to record the acoustic signal generated during experiment. Lock-in \#1 and lock-in \#2 are referred to each other by using the same reference signal. Details of the detection of shear-force and acoustic signals will be discussed in latter chapters, according to objectives of experiments. If there are more than two signals to be measured, a lock-in SR844 will be used along with these two SR850 lock-in amplifiers. 


\section{Chapter 4. Field-Programmable Gate Array in Distance Regulation}

\subsection{Introduction of Field-Programmable Gate Array}

Field-programmable gate arrays (FPGAs) are semiconductor devices whose features and functions can be configured after manufacturing. An FPGA is composed of an array of logic blocks linked to each other through an interconnect network. In contrast to most digital integrated circuits, where the functionality of the device is frozen in silicon, FPGAs can be reprogrammed and upgraded to meet customer's changing needs. One big advantage of the FPGA over a general-purpose processor is its ability to perform data processing tasks in a massively parallel fashion. FPGAs therefore can handle very fast computing. Due to the rapid prototyping features and the flexibility, FPGA technology has gained rapid acceptance over the past decade. FPGAs are now being used in a wide range of application areas such as image and signal processing, wired and wireless telecommunications, space and aircraft embedded control systems.

Herein, using FPGA for the implementation of data acquisition tasks is highlighted. The data acquisition device uses an FPGA-based system timing controller to make all analog and digital I/Os configurable for application-specific operation. By programming the FPGA via Nation Instruments LabVIEW FPGA Module, the custom highperformance data acquisition tasks can easily be implemented. Additionally, because of the parallel architecture of the FPGA, a control algorithm can have a very short execution time, allowing a large amount of data being captured at a very high rate in real time. With the direct memory access (DMA) capabilities in the LabVIEW FPGA module, a direct 
link for data on the FPGA to RAM on the host machine is achieved, improving datalogging efficiency and making data immediately available for analysis and visualization. This feature of high-speed data transfer makes FPGAs particularly adapted in the control of any scanning probe microscope (SPM) system; because SPM control loops usually require a rapid response, in a fraction of a millisecond, to changes in probe-sample interactions.

The FPGA is a function block for data collection. Acquiring real-world analog signals from transducers, and generating analog signals for output signals to control devices typically requires analog-to-digital (A/D) and digital-to-analog (D/A) converters. The motion control of the acoustic/shear-force microscope system was implemented on a multifunction data acquisition (DAQ) card NI PCI-7831R (National Instruments, Austin, TX, USA). The card contains (1) an Virtex-II 1M gate FPGA onboard chip whose functionality can easily be changed using the LabVIEW software package; (2) eight independent, 16-bit analog inputs with bandwidths of $200 \mathrm{kHz}$; (3) eight independent, 16bit analog outputs with bandwidths of $1 \mathrm{MHz}$; and (4) 96 digital lines at rate of $40 \mathrm{MHz}$. The FPGA has a sample rate of $200 \mathrm{kSamples/s,} \mathrm{giving} \mathrm{a} \mathrm{measured} 5 \mu$ s loop period. The parallelism of the FPGA enables all of the analog I/O and digital I/O ports to be accessed simultaneously.

Despite the fact that the FPGA-DAQ card is a powerful tool, it has limitations that make a highly precise control of all aspects of the microscope difficult. For example, the resolution of the voltage outputs becomes a major problem when they are used in 
microscope's motion control. An additional analog circuitry is therefore developed. More details regarding the analog circuit will be discussed in the following sections.

\subsection{Precise Z-Axis Position Control via FPGA-DAQ Card}

The precise positioning control of probe in the vertical (Z) direction is performed by a piezo-actuated linear nanopositioning stage Nano-OP65 (Mad City Labs Inc., Madison, WI, USA), which offers a 65- $\mu \mathrm{m}$ range of motion in single axis. The stage has integrated position sensor with subnanometer resolution. The readout from the position sensor controls the input voltage on the piezoactuator through a closed loop feedback, which eliminates the effects of inherent creep and hysteresis due to the piezoelectric materials. The electronic controller of Nano-OP65 piezo stage also provides standard analog control inputs via front panel BNC's. The standard analog position command signal is configured for a $0-10 \mathrm{~V}$ input. Typically the analog output voltage of the FPGA-DAQ card is rated for $\pm 10 \mathrm{~V}$. It opens the opportunity to drive the piezoactuator to move through the FPGA-DAQ card.

Since the analog outputs on the FPGA-DAQ card have a 16-bit resolution, it is determined that the best positioning resolution attainable with the $65-\mu \mathrm{m}$ range of displacement will result in 65000/32767 or $2 \mathrm{~nm}$ per analog voltage division, theoretically, not including the output noise which will be further discussed in the next section. The performance of Z-motion stage would be limited by the external driving voltage given from the FPGA-DAQ card. Therefore, a summing operational amplifier (op amp) is designed in order to reach the desired resolution. Instead of using single analog 
output, two analog outputs are dragged from the FPGA-DAQ card, and then summed by the additive circuit before applying to the controller of the piezo stage. As presented in Figure 4.1, one of analog outputs feeds into a voltage divider. Indeed it was able to increase the positioning resolution, but the entire range of the driving voltage and thereby the displacement of piezoelectric stage would be proportionally diminished. To circumvent this issue, the op amp circuit is used to add the other analog output for level shifting. Ultimately, the driving signal of the piezoactuator is the output from the additive amplifier, which is the result of summing one signal (fine signal) that has a reduced sweep range but an increased resolution, and the other signal (coarse signal) used to offset the former by an amount equal to its full range of sweep.

Level shifting

Long Rang Motion, Low Resolution

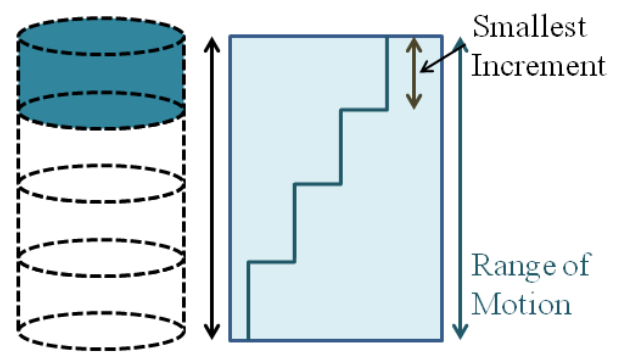

Voltage Dividing

Short Rang Motion, High Resolution

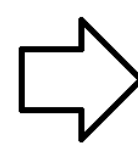

Summing Amplifire

Resulting Output Long Rang Motion, High Resolution
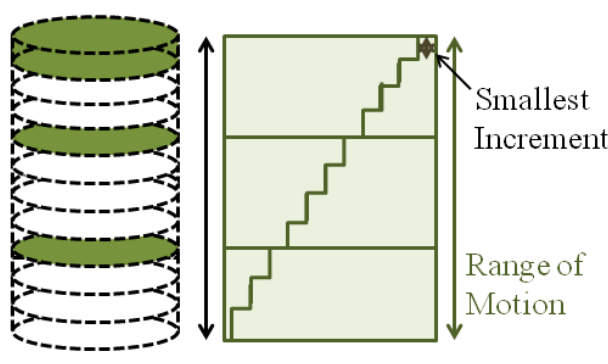

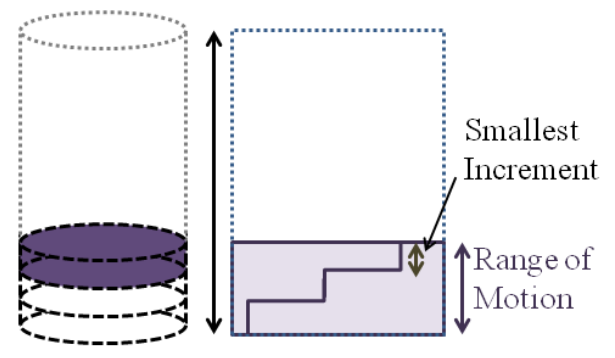

Figure 4.1: Strategy used to attain long range of motion with high resolution. 
Figure 4.2 illustrates the schematic of the analog summing amplifier circuit used in displacement control, as well as corresponding voltage measurement on each port. The ratio of two input resistors $\mathrm{K}$ determines the number of divisions relevant to the best resolution of Z-axis motion. The FPGA-DAQ card is programmed to return the fine signal to its minimum value (-32766) once the maximum value (32767) is reached, and to shift the reset fine signal based on the coarse signal. The amount of offset is also determined by the value of $\mathrm{K}$. The synchronization between the fine and coarse signals has to be carefully implemented. In the case of $\mathrm{K}$ being set to be 100 , driving the piezo stage using the FPGA-DAQ card, along with the summing op amp circuit, a theoretical resolution of $0.02 \mathrm{~nm}$ in $\mathrm{Z}$ axis would be attained.
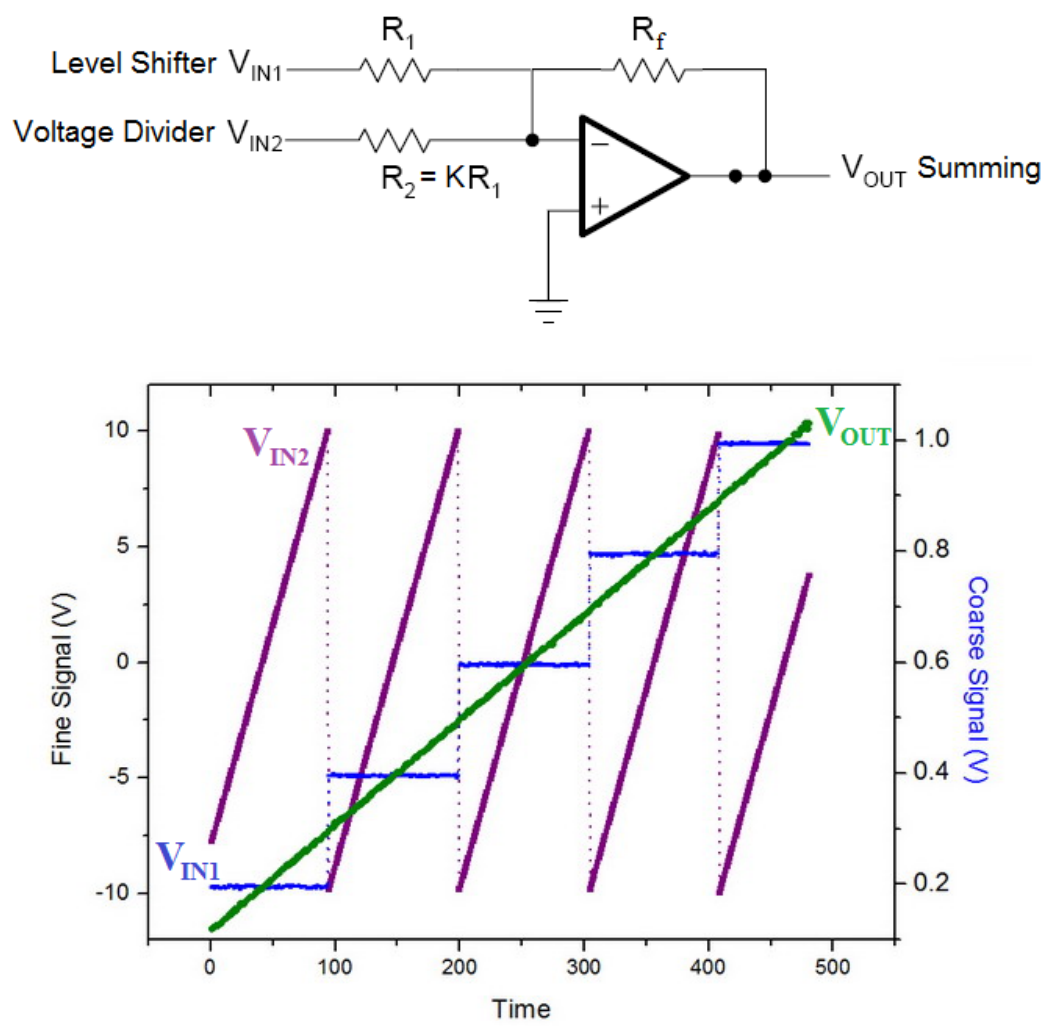

Figure 4.2: Schematic of the summing amplifier and corresponding voltage signal on each port. 


\subsection{Noise Level of Analog Outputs on FPGA-DAQ Card}

As mentioned above, the ideal resolution of displacement under the FPGA-DAQ card control is $0.02 \mathrm{~nm}$. However, the actual resolution is worse because of the noise. One main noise source is the FPGA-DAQ card itself. The card has different noise levels for D/A conversion and A/D conversion, outlined in the product specification data sheet. The manufacturer specifies the input noise to be $\pm 7.78 \mathrm{mV}$ and the output noise as $\pm 5.88 \mathrm{mV}$ for NI PCI-7831R card. In addition, it will also be useful to think about the voltage noise in terms of its working environment. Thus, the actual noise levels will be determined by measuring the signals from the analog outputs on the FPGA-DAQ card via an oscilloscope. As running the FPGA-DAQ card on the host computer, a 15-mV noise was measured on both fine and coarse signals. When the output voltages are used to directly drive piezoactuators, this noise level will be a limiting factor in increasing the precision of displacement in $\mathrm{Z}$ axis. Adding low-pass filters to filter out undesired noises becomes essential.

Low-pass filters are used whenever high frequency components must be removed from a signal. In the case of positioning control, both fine and coarse signals from the analog outputs would be almost treated as DC signals. If a low-pass filter with appropriate cutoff frequency is placed at each output channel, it allows the desired signal to pass, and rejects unwanted signals at high frequencies, thereby reducing the overall noise level. Active low-pass filters were chosen instead of LRC passive filters. Active filters are circuits that use an op amp as the active device in combination with resistors and capacitors to provide an LRC-like filter performance at low frequencies. The only 
reason of not using passive filters was LRC filters cannot effectively remove noise signals from the analog outputs of the FPGA-DAQ card. In addition, high-order filters were used for the purpose of having a steeper amplitude response roll-off. The more poles in the active filter the more attenuation at a given frequency in the stop band.

Finally, a fourth-order unity-gain Butterworth low-pass filter with $1 \mathrm{kHz}$ cutoff frequency, followed by a first-order passive RC low-pass filter with $100 \mathrm{kHz}$ cutoff frequency, was used to diminish the noise, and its schematic is shown in Figure 4.3.

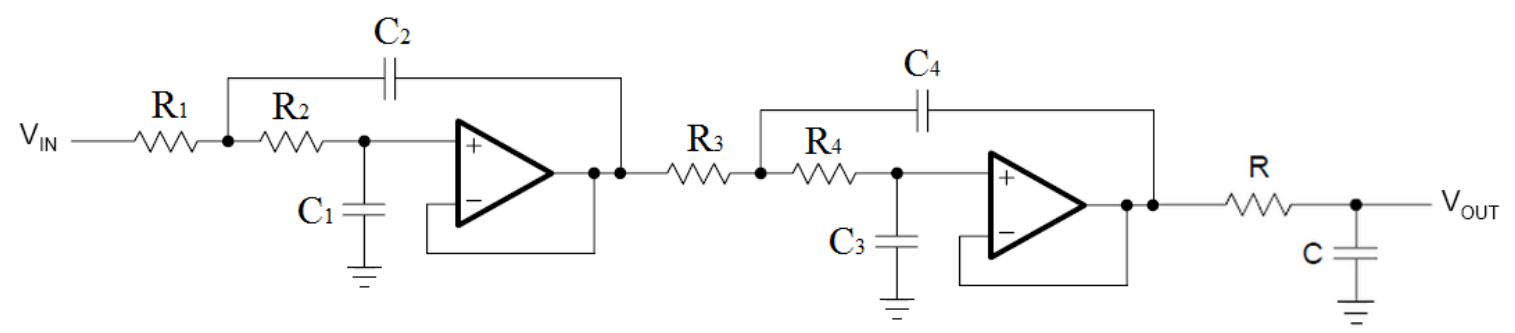

Figure 4.3: Schematic of low-pass filter used to diminish the unwanted noise from the analog outputs of FPGA-DAQ card.

First of all, let us determine the values of the resistor (R) and the capacitor (C) used in the first-order passive filter. For any specified capacitor value C, the resistor value R can be calculated through:

$$
R=\frac{1}{2 \pi f_{c} C}
$$

where $f_{c}$ represents the cutoff frequency of the filter. In this case of $\mathrm{C}=0.15 \mu \mathrm{F}$, and $f_{\mathrm{c}}=$ $100 \mathrm{kHz}$, and

$$
R=\frac{1}{2 \pi f_{c} C}=\frac{1}{2 \pi \cdot 1 \cdot 10^{3} \cdot 15 \cdot 10^{-6}}=10.6 \Omega
$$

Therefore, the value of $\mathrm{C}$ is $0.15 \mu \mathrm{F}$ and the closest $1 \%$ value of $\mathrm{R}$ is $11 \Omega$. 
And secondly, let us think about the values of resistors $\left(\mathrm{R}_{1}, \mathrm{R}_{2}, \mathrm{R}_{3}\right.$ and $\left.\mathrm{R}_{4}\right)$ and capacitors $\left(\mathrm{C}_{1}, \mathrm{C}_{2}, \mathrm{C}_{3}\right.$ and $\left.\mathrm{C}_{4}\right)$ used in the fourth-order active filter. In fact, the fourthorder filter is formed by cascading two second-order filters together. To start with, the coefficients for a fourth-order Butterworth filter are given by:

First partial filter $\mathrm{a}_{1}=1.8478 \mathrm{~b}_{1}=1.0000$

Second partial filter $a_{2}=0.7654 b_{2}=1.0000$

For each partial filter, the specified capacitor values $C_{1}$ and $C_{2}$ must meet the condition:

$$
C_{2} \geq C_{1} \frac{4 b_{1}}{a_{1}^{2}}
$$

The resistor values $\mathrm{R}_{1}$ and $\mathrm{R}_{2}$ can be calculated through:

$$
R_{1,2}=\frac{a_{1} C_{2} \mp \sqrt{a_{1}^{2} C_{2}^{2}-4 b_{1} C_{1} C_{2}}}{4 \pi f_{c} C_{1} C_{2}}
$$

In the first filter stage, with $\mathrm{C}_{1}=0.015 \mu \mathrm{F}, \mathrm{C}_{2}=0.018 \mu \mathrm{F}$ meets the restriction. For $f_{\mathrm{c}}=$ $1 \mathrm{kHz}$, calculating the values for $\mathrm{R}_{1}$ and $\mathrm{R}_{2}$ obtains,

$$
\begin{gathered}
R_{1}=\frac{a_{1} C_{2}-\sqrt{a_{1}^{2} C_{2}^{2}-4 b_{1} C_{1} C_{2}}}{4 \pi f_{c} C_{1} C_{2}}=8.3 \mathrm{k} \Omega \\
R_{2}=\frac{a_{1} C_{2}+\sqrt{a_{1}^{2} C_{2}^{2}-4 b_{1} C_{1} C_{2}}}{4 \pi f_{c} C_{1} C_{2}}=11.3 \mathrm{k} \Omega
\end{gathered}
$$

The same calculation would be applied to the second filter stage for $f_{\mathrm{c}}=1 \mathrm{kHz}$. Specifying that $\mathrm{C}_{3}=0.015 \mu \mathrm{F}$ and $\mathrm{C}_{4}=0.1033 \mu \mathrm{F}$, calculating $\mathrm{R}_{3}$ and $\mathrm{R}_{4}$ obtains,

$$
R_{3}=\frac{a_{2} C_{4}-\sqrt{a_{2}^{2} C_{4}^{2}-4 b_{2} C_{3} C_{4}}}{4 \pi f_{c} C_{3} C_{4}}=3.7 \mathrm{k} \Omega
$$




$$
R_{4}=\frac{a_{2} C_{4}+\sqrt{a_{2}^{2} C_{4}^{2}-4 b_{2} C_{3} C_{4}}}{4 \pi f_{c} C_{3} C_{4}}=4.4 \mathrm{k} \Omega
$$

In summary, the actual values of resistors and capacitors used in filter design are:

\begin{tabular}{|c|c|c|c|c|}
\hline \multicolumn{2}{|c|}{ 1st Filter Stage } & \multicolumn{2}{c|}{ 2nd Filter Stage } & $\begin{array}{c}\text { 3rd Filter } \\
\text { Stage }\end{array}$ \\
\hline $\mathrm{R}_{1}=8.25 \mathrm{k} \Omega$ & $\mathrm{C}_{1}=0.015 \mu \mathrm{F}$ & $\mathrm{R}_{3}=3.65 \mathrm{k} \Omega$ & $\mathrm{C}_{3}=0.015 \mu \mathrm{F}$ & $\mathrm{R}=11 \Omega$ \\
\hline $\mathrm{R}_{2}=11.3 \mathrm{k} \Omega$ & $\mathrm{C}_{2}=0.018 \mu \mathrm{F}$ & $\mathrm{R}_{4}=4.4 \mathrm{k} \Omega$ & $\mathrm{C}_{4}=0.1033 \mu \mathrm{F}$ & $\mathrm{C}=0.15 \mu \mathrm{F}$ \\
\hline
\end{tabular}

Table 4.1: Values of resistors and capacitor used in the low-pass filter. 


\section{Chapter 5. Characterization of Mesoscopic Fluid Films via Shear-Force/Acoustic Near-Field Microscopy}

\subsection{Introduction}

To date, one of the most common techniques to monitor the position of the probe relative to the sample surface in a scanning probe microscope (SPM) is based on the socalled shear force distance control mechanism. Briefly speaking, the term shear force refers to the probe-sample interactions that cause net decrease in the amplitude of an SPM probe's oscillation that is conveniently driven at its resonance frequency in a direction parallel to a sample surface. As the probe is brought within $\sim 10 \mathrm{~nm}$ of the surface at ambient conditions, a gradual decrease in the amplitude of probe oscillation is observed, which is generally attributed to short-range forces between the probe and the sample $^{27,68}$.

The probe-sample distance-dependent shear force is widely used as a feedback regulation of the probe's vertical position; hence an understanding of its origin is very desirable in SPM. However, the exact nature of the shear-force interaction still remains a subject of discussion. Several possible interaction mechanisms have been proposed as an explanation. It is believed that a complete vanishing of the oscillations is an indication that the probe has come into a mechanical contact with the surface. Gregor et al. proposes a nonlinear bending force model, where the tip intermittently knocks on the surface as in the tapping mode due to a (typically unavoidable) slight tilt of the probe's axis relative to the normal to the surface. The intermittent knocking leads to increases in the resonance frequency of the probe, as well as the damping of its oscillation ${ }^{69}$. Unfortunately, this 
study uses large amplitude of vibration in the order of $200 \mathrm{~nm}$, which is not typical in most SPM applications. The shear-force mechanism has also been undertaken from the perspective of interfacial friction (wear-free friction phenomena) ${ }^{36,70}$. The study provides evidence that even under vacuum conditions the measured viscous and elastic shear stress is directly attributed to a third body filling in the probe-sample gap. Additionally, it is accepted that the effects originating from electrostatic forces, van der Waals forces, capillary force, and viscosity force have effects on the probe's oscillation. At ambient conditions, most of materials are hydrophilic enough to attract the air humidity and other hydrocarbon molecules, leading to a fluid-like layer of a few-nanometers thickness conventionally termed contamination layer. There exists experimental evidences showing that the probe oscillating within the thin contamination layer undergoes a damping in its motion $^{71,72}$. However, the most surprising finding is that the probe's resonance frequency increases upon its interaction with the contamination layer, opposed as what is expected from pure damping effect.

Herein we study the probe-sample interaction by performing a series of experiments using our shear-force/acoustic near-field microscope. Our analysis relies on the simultaneous measurements of electrical signals generated by a tuning fork (TF) and an acoustic sensor (AS) as a function of the probe-sample separation while a tip approaches towards and/or retracts from a sample surface. The key point to be highlighted here is to investigate the potential benefits of having an extra technique (the acoustic signal) to characterize shear forces. Systematic measurements are carried out by varying the experimental conditions, such as tip geometry, environmental humidity, chemical 
properties of sample surfaces, and the velocity of the tip's motion in vertical direction. Information about the probe-sample interaction is extracted from approach/retraction curves, providing both shear-force and acoustic interpretations of the influence of contamination layers on the distance regulation in SPM operation.

\subsection{Experimental Section}

A sharp gold tip fabricated using electrochemical etching was manually mounted to one of prongs of a quartz tuning fork (TF). The tip usually protrudes $<1 \mathrm{~mm}$ out of the end of the prong. Small pieces $(1 \mathrm{~cm} \times 1 \mathrm{~cm})$ of sample substrates (silicon wafers and mica disks) were subjected to a treatment (i.e., cleaning or surface modification) before use. Typically, the silicon wafers were cleaned by immersion in Piranha solution and dried under $\mathrm{N}_{2}$. The mica disks were cleaned using acetone and IPA with ultrasonic agitation for at least 30 min and dried by blowing $\mathrm{N}_{2}$. Usually, a few layers on the top side of the cleaned mica sheet are peeled off to obtain an extremely flat surface. Sometimes, various cleaning methods and chemical modifications of substrate surfaces were carried out to meet the needs of experiments (details regarding the experimental procedure will be introduced in specific sections). Subsequently, the freshly prepared sample was loaded to the top of an acoustic sensor (SE40-Q, Dunegan Engineering Consultants, Inc.), which is mainly used to detect the acoustic signal generated during experiments. In order to improve the quality of acoustic signal, Dow Corning high vacuum grease was used to increase the coupling efficiency of sound wave propagation when the bottom surface of the sample was in intimate contact with the top 
surface of the acoustic sensor. A small chamber covers the stage of the shearforce/acoustic microscope. The humidity can be varied by such means as filling the chamber with a mixture of humid and dry $\mathrm{N}_{2}$, or putting a saturated salt solution into the chamber.

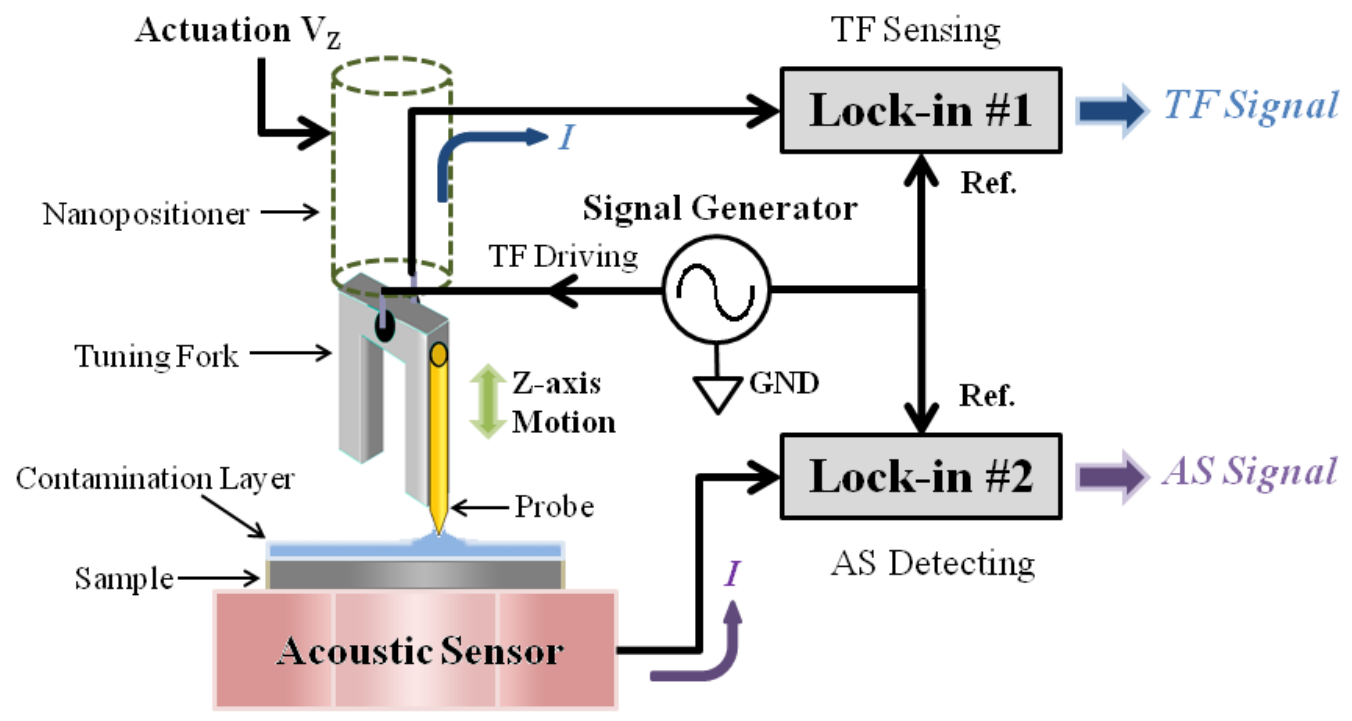

Figure 5.1: Schematic of the experimental setup used to detect the TF signal along with the acoustic signal as the gold tip approaches to and withdraws from the sample surface.

The setup of the shear-force/acoustic microscope is schematically presented in Figure 5.1. The system can be broken down into two distinct sections: the microscope stage and the detection system. The microscope stage sits on a floating optical bench to minimize mechanical perturbations. A piece of rubber cushion is placed between the stage and the optical bench to further reduce undesigned disturbances. The probe can approach towards or retract from the sample surface under coarse and fine adjustments of the probe-sample distance, which is monitored through a CCD camera. Coarse control is performed using a set of fine-pitch screws (Newport; model: AJS100-2, travel: $50.8 \mathrm{~mm}$, sensitivity: 0.71 
$\mu \mathrm{m}$ ), which allow a rapid movement of a few millimeters with precision in the order of micrometer. If the probe-sample distance falls into the range of $50 \mu \mathrm{m}$, fine control is carried out using a piezo-actuated nanopositioner (Nano-OP65, Mad City Labs, Inc.) that provides $65 \mu \mathrm{m}$ range of motion with nanometer accuracy. More importantly, the nanopositioner can be operated in close loop so as to eliminate the hysteresis arisen from the piezoelectric components.

In operation, a sinusoidal wave from the internal reference source of a lock-in amplifier (Lock-in \#1) (SR850 model, Stanford Research System) is used to driven the TF. The TF is excited to vibrate at its resonance frequency in a direction parallel to the sample surface. The acoustic sensor is located under the sample and directly connected to the other lock-in amplifier (Lock-in \#2) (SR850, Stanford Research System). These two lock-in amplifiers share the same reference signal that is the excitation source of the TF.

Detection of the probe's oscillation amplitude is implemented based on the piezoelectric effect of quartz materials which generate a piezoelectric voltage proportional to the amplitude of the mechanical vibration. A quantitative estimation of the vibration amplitude can be done by applying the harmonic approximation model ${ }^{73}$, which states that $\Delta I=4 \pi f \alpha u_{0}$, where $\Delta I$ is the change in current, $\alpha$ is the piezoelectromechanical coupling constant, and $u_{0}$ is the root-mean-square (rms) amplitude of the electric current of the TF. In our case, at resonance an approximate relationship between $\Delta I$ and $u_{0}$ states that if $\Delta I=10 \mathrm{nA}$ rms then $u_{0}=4 \mathrm{~nm} \mathrm{rms}$.

As the tip is approaching to the sample surface, variations of electric current generated by the TF and the acoustic sensor are being simultaneously recorded as a 
function of the probe-sample distance and subsequently plotted as the approach curves: one reflects the effect of shear forces acting on the tip (the TF signal), the other is the corresponding response of the acoustic sensor (the acoustic signal). To complete one experimental cycle, the retraction curves of the TF and acoustic signals are also stored while the probe is retracting from the surface.

\subsection{Results and Discussions}

Variations of the TF and acoustic signals during the approach and retraction processes can be considered as a reflection of the probe-sample interaction, which is influenced by several factors. Herein we are concentrated on the impacts of parameters such as tip geometry, relative humidity of the environment, the nature of sample surfaces, and velocity of the tip's movement on the measured approach/retraction curves. Their effects will be presented and discussed in turn.

\subsubsection{Uniformity of the Contamination Layer}

In general, the tip is randomly positioned on the sample surface for obtaining approach/retraction curves. Does the selection of testing sites affect our measurement? To answer this question, we carried out a simple experiment which could approximately provide idea regarding how the fluid-like contamination layer distributes on top of the solid surface. Here our discussion is based on the measurements of the TF and acoustic signals on a freshly cleaved mica surface using a sharpened gold tip at 25\% humidity. 
A piece of mica (highest quality grade V1 mica, Ted Pella Inc.) serves as the sample substrate in order to avoid uncertainties resulted from surface topography. Prior to loading the mica disk, few layers were peeled off so as to obtain a clean and flat surface. However, the surface will be contaminated soon if it is exposed to the ambient atmosphere. The time-dependent growth of contamination layer has been reported in literatures ${ }^{72,74}$. In our experiment, five testing sites on the sample surface were arbitrarily chosen for measurements. For each testing site, five approach/retraction curves were obtained by moving the tip down and up at the same speed of $2 \mathrm{~nm} / \mathrm{s}$. The tip would move to the next place as long as the five trials were completed. The approach process stopped and the retraction process immediately began once the amplitude of tip's vibration dropped to $75 \%$ of the initial value. The tip was imaged with an electron scanning microscope (SEM) before and after experiment; no essential damage was visible on the tip due to the approach/retraction operation. 


\section{Testing Site \#3}
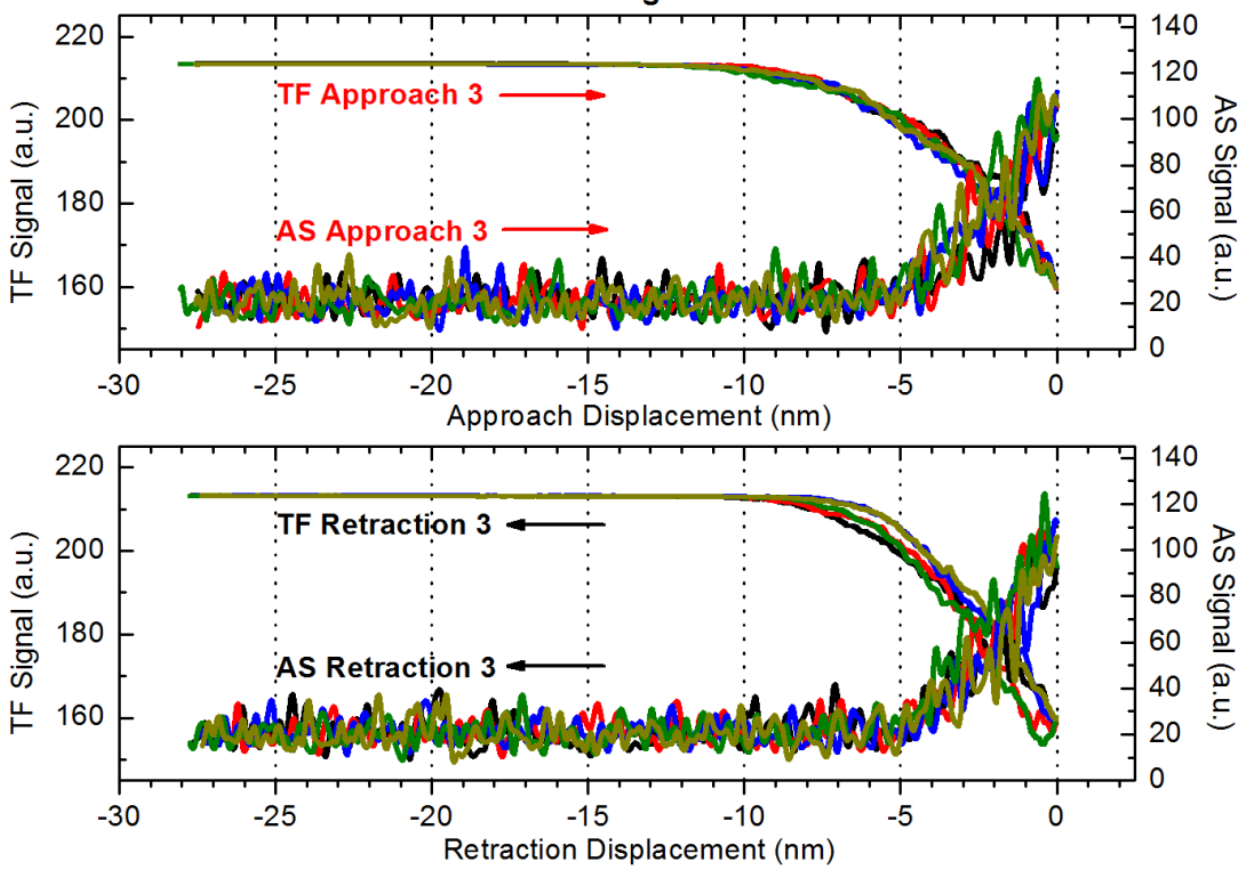

Figure 5.2: Five independent trials of approach (upper) and retraction (lower) measurements preformed on the same testing site. Different colors represent different trials.

Figure 5.2 shows the five measurements on the same testing site. The "zero" coordinates of the horizontal axis represents the end of approach curves and the beginning of retraction curves. Despite the fact that these five traces slightly differ from each other, they have the same characteristics such as the trend of decreasing the TF amplitude and increasing the acoustic signal, as well as the length of transition from the maximum to the minimum. Subsequently, we summarize the experimental results obtained from five different testing sites in Figure 5.3: individual curve is obtained by taking an average of five approach/retraction curves measured on the same position. Since the testing sites were randomly selected, the high similarity among different curves 
indicates that the contamination layer almost uniformly covers the sample surface within the area that we tested.

Summary of Results on 5 Testing Sites

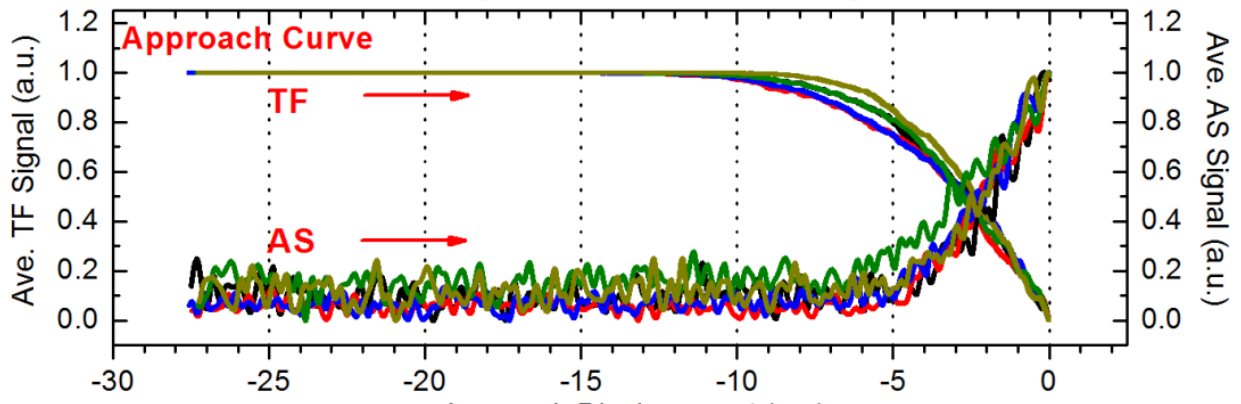

Approach Displacement (nm)

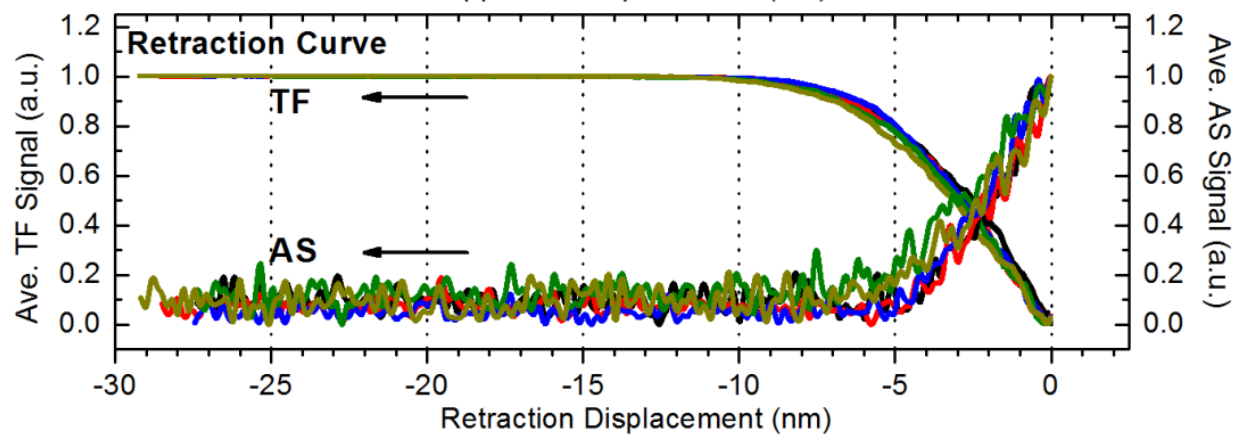

Figure 5.3: A summary of approach (upper) and retraction (lower) measurements preformed on five different testing sites. Different colors represent the tip being placed at different locations. Each curve is an average of five independent trials.

\subsubsection{Effect of Tip Geometry}

Experiments were performed on a bare silicon wafer using two different gold tips when the environmental humidity was $25 \%$. Prior to use, the silicon substrate was cleaned using Piranha solution. Approach/retraction curves were obtained by bringing the tip close to and away from the sample with a constant speed of $1.5 \mathrm{~nm} / \mathrm{s}$. Figure 5.4 shows the scanning electron microscope (SEM) images of a chubby tip (left) and a sharp tip (right), respectively. The chubby tip has a larger surface area of the tip apex compared 
to the sharp tip. It was found that the resonance frequency of the chubby tip was 31394 $\mathrm{Hz}$ when it was driven to vibrate in air. As a driving voltage of $14 \mathrm{mV}_{\text {rms }}$ was applied, its oscillation amplitude was then estimated to be $\sim 8 \mathrm{~nm}$, which is quite small compared to the tip radius. The same attempt was repeated on the sharp tip as well: as the sharp tip oscillated in air, its resonance frequency was $31272 \mathrm{~Hz}$ and. When applying the same driving voltage (14 $\mathrm{mV}_{\mathrm{rms}}$ ), the oscillation amplitude was $\sim 9 \mathrm{~nm}$, which is still smaller than the tip radius.

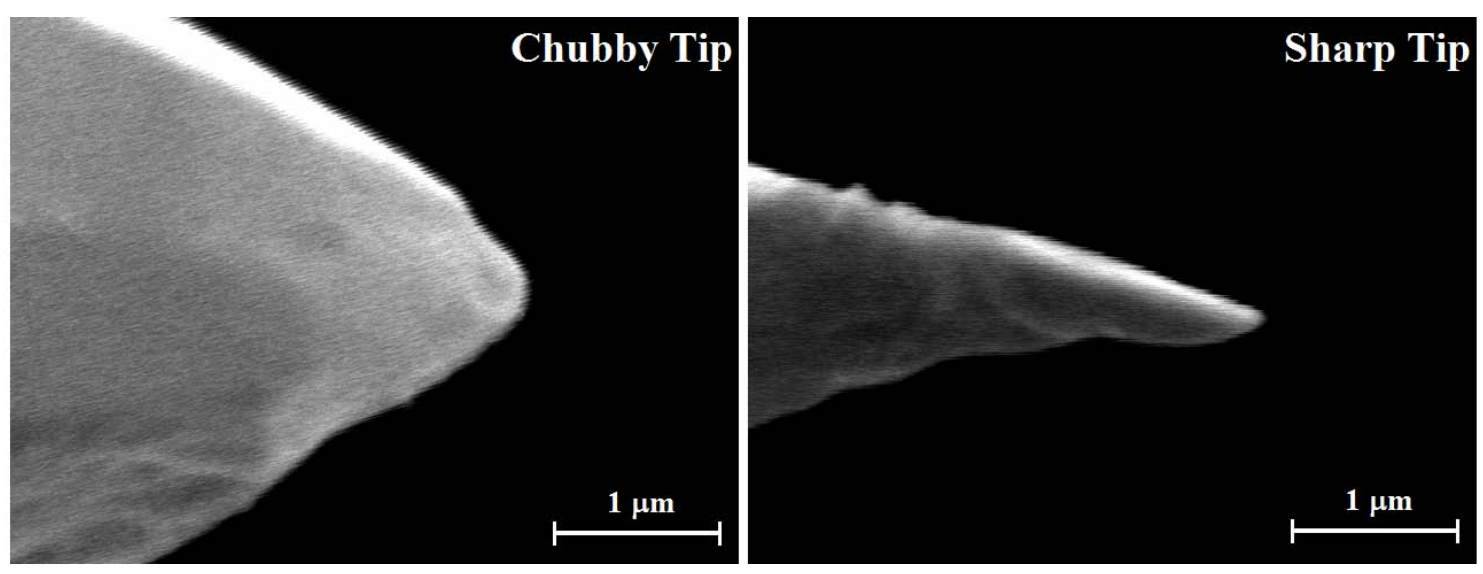

Figure 5.4: Scanning electron microscope images of a chubby gold tip (left) and a sharp gold tip (right). The scale bar represents $1 \mu \mathrm{m}$.

In one experimental cycle, as the chubby tip moves in the vertical (Z-axis) direction, variations of the TF and acoustic signals are plotted with the change in the tip's position relative to the sample surface. In Figure 5.5, the "zero" coordinates of the horizontal axis is arbitrarily chosen as the position where the TF signal starts to drop. The amplitude of the TF signal remains a constant when the probe is far away from the sample. The tip oscillating in air generates sound waves shown as a nonzero background of the acoustic signal. In Figure 5.5a, when the tip moves closer to the sample, it experiences an abrupt 
disturbance labeled as point "A", leading to a significant decrease in the amplitude of the TF signal. The formation of a water meniscus is thought to have a contribution to the sudden fall of the TF signal ${ }^{75}$. Due to the presence of a mesoscopic fluid-like layer formed by absorbing water molecules from the ambient, a meniscus wrapping around the tip leads to a capillary force when the tip enters the liquid film. It restricts the lateral motion of the tip and causes the steep drop in the amplitude of the TF signal. However, there is no corresponding change in the acoustic signal above the noise level being detected. Such a weak sound signal may be hidden by the background at the air/water interface. On further tip approach its oscillation monotonically decreases mainly due to the contribution of viscoelastic interaction with the mesoscopic film. The observation of the TF signal decreasing with reducing the probe-sample separation is a combination of the damping of the oscillation amplitude and the blue-shift of the resonance frequency. However, the possibility of the tip apex in intermittent contact with the surface at a small probe-sample distance cannot be completely eliminated, which also causes the decrease in the TF signal. Besides, other forces such as electrostatic and van der Waals forces can also make the TF signal decrease.

Furthermore, we can divide the entire transition region of the measured signals into different parts based on the relationship between TF and acoustic signals: they are positively correlated, which means the TF amplitude decreases along with a decrease in the acoustic signal; and they are negatively correlated, which means the TF amplitude decreases whereas the acoustic signal increases. Acoustic waves are generated because of the tip's mechanical vibration in a medium. In general cases (i.e., in air), the amplitude of 
the acoustic signal is proportional to the amplitude of the probe's oscillation. As shown in Figure 5.5a, after entering the liquid film, the vibration amplitude of the chubby tip is much reduced by water wrapping round it, leading to less sound detected by the acoustic sensor (in the region from $0 \mathrm{~nm}$ to $\sim 10 \mathrm{~nm}$ ). As the vibrating tip penetrates deeper into the fluid, the phase lagging between the tip end and the liquid layer becomes more pronounced. Hence the generation of sound waves is more efficient to compensate the loss due to the decrease in the tip's oscillation amplitude. The net effect is an increase in the acoustic signal. On the other hand, the knocking of the tip on the sample is also a potential factor that makes the acoustic signal rise up. It cannot be simply ruled out only according to the TF and acoustic measurements. At the end of approach, the tip in contact with the hard surface is a plausible explanation for the final decreases in both TF and acoustic signals. A similar analysis can also be applied to the retraction measurements shown in Figure 5.5b. We notice that hysteresis between the approach and retraction curves is not significant in the liquid layer. It becomes remarkable only when the tip leaves the interface into the air. The sudden jump, labeled as point " $\mathrm{B}$ " (demonstrated in the insertion), at the end of the TF retraction curve indicates that the tip is pulled out of the boundary of the liquid region. 

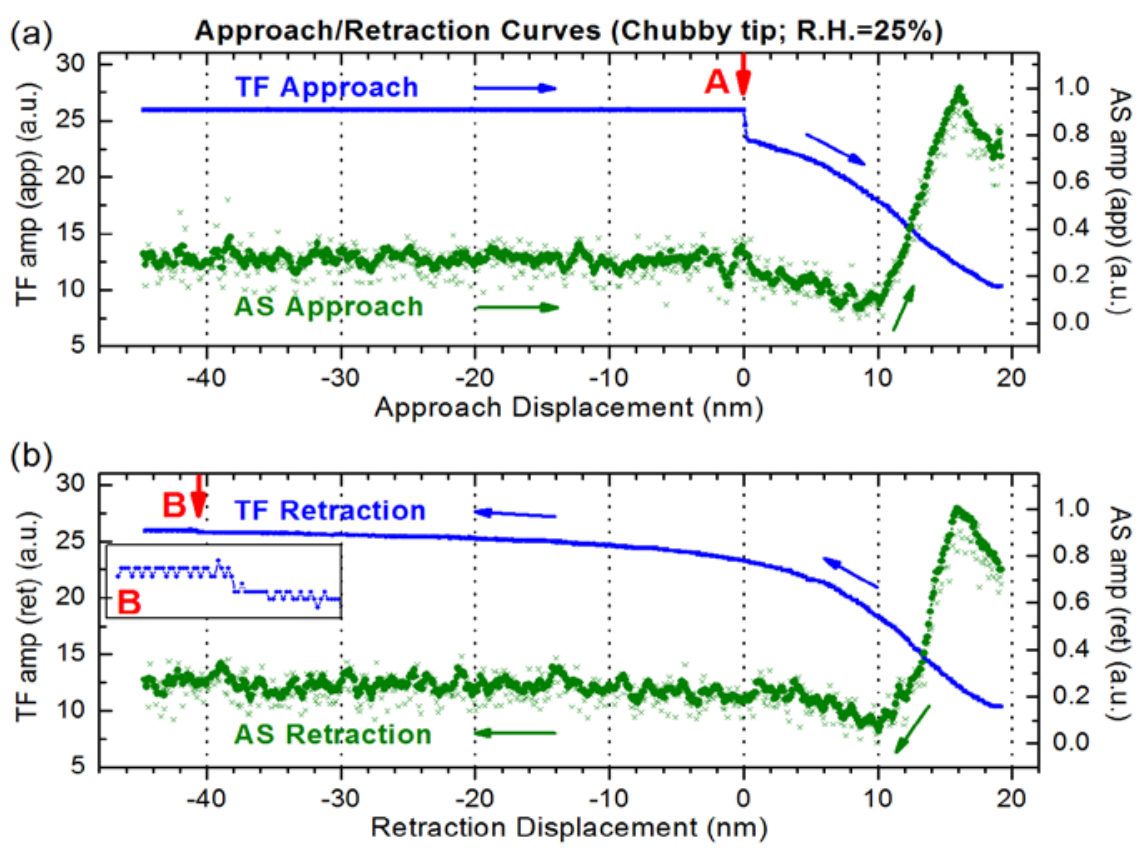

Figure 5.5: Approach (a) and retraction (b) curves of TF (blue) and acoustic (green) signals obtained with the use of a chubby tip and a bare silicon wafer (humidity: 25\%). Insertion: The detail of the TF signal at point B.

The same experiment described above was repeated by replacing the chubby tip by the sharp one. Approach/retraction curves shown in Figure 5.6 illustrate variations of the TF and acoustic signals when changing the tip’s vertical position relative to the sample. The abrupt drop on the TF approach curve in Figure 5.5a is not detectable in the experiment using the sharp tip instead of the chubby one. It is attributed to the small radius of the sharp tip which has impact on the dimension of the formed meniscus. In comparison to Figure 5.5a, another difference is that there is no positive correlation between the TF and acoustic signals displaying in Figure 5.6. As we mentioned above, sound is produced by the vibrating probe; in the absorbed film, the interaction between the probe and the fluid depends on the tip geometry. The acoustic measurement shows higher sensitivity near the liquid/solid (mesoscopic fluid/hard sample) interface than at 
the gas/liquid (air/mesoscopic fluid) interface. For a sharp tip, the reduction of the acoustic amplitude in response to the decrease in the probe's oscillation amplitude may not be visible above the noise level. We also found that the region of negative correlation extends out while using the sharp tip. Compared to the chubby tip, the oscillation amplitude of the sharp tip is not substantially attenuated in a short distance due to less tip surface area in contact with the liquid. Additionally, the acoustic signal does not change obviously at the end of approach in Figure 5.6a. A potential mechanism to generate large acoustic output is the sliding of the tip on the surface. Retraction curves of the TF and acoustic signals were obtained by withdrawing the sharp tip from the sample. Unlike the long tail on the TF curve shown in Figure 5.5b, the sharp tip travels a shorter distance until it completely leaves the contamination layer, which is labeled as point "B" in Figure 5.6b. It can also be attribute to less interaction between the probe and the fluid when the tip radius gets smaller. 

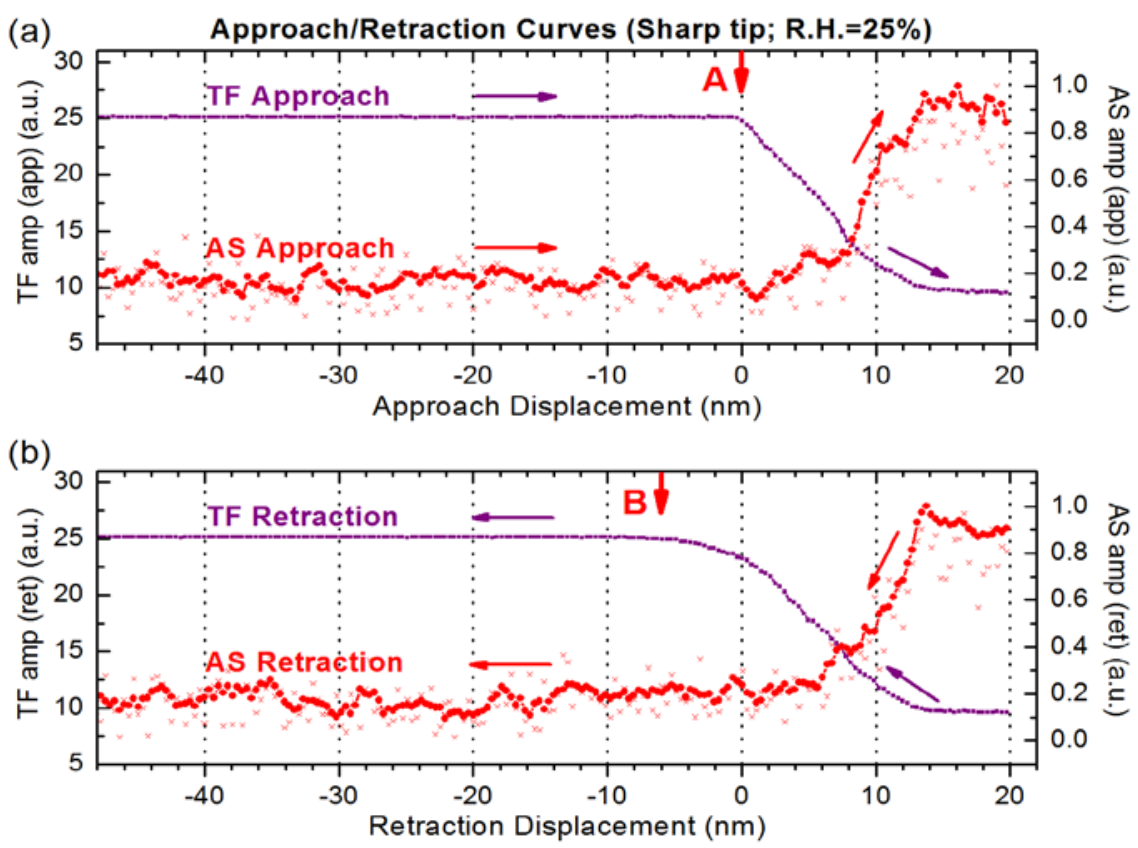

Figure 5.6: Approach (a) and retraction (b) curves of TF (purple) and acoustic (red) signals obtained with the use of a sharp tip and a bare silicon wafer (humidity: 25\%).

\subsubsection{Effect of Environmental Humidity}

The influence of relative humidity (R.H.) was studied by carrying out the same experiments as described in previous section while raising environmental humidity to 55\%. Figures 5.7 and 5.8 show variations of the TF and acoustic signals plotted as a function of the probe-sample separation for the same chubby and sharp tips previously used, respectively. The "zero" coordinates of the horizontal axis is defined as the point where the TF signal starts to decrease during the approach process. 

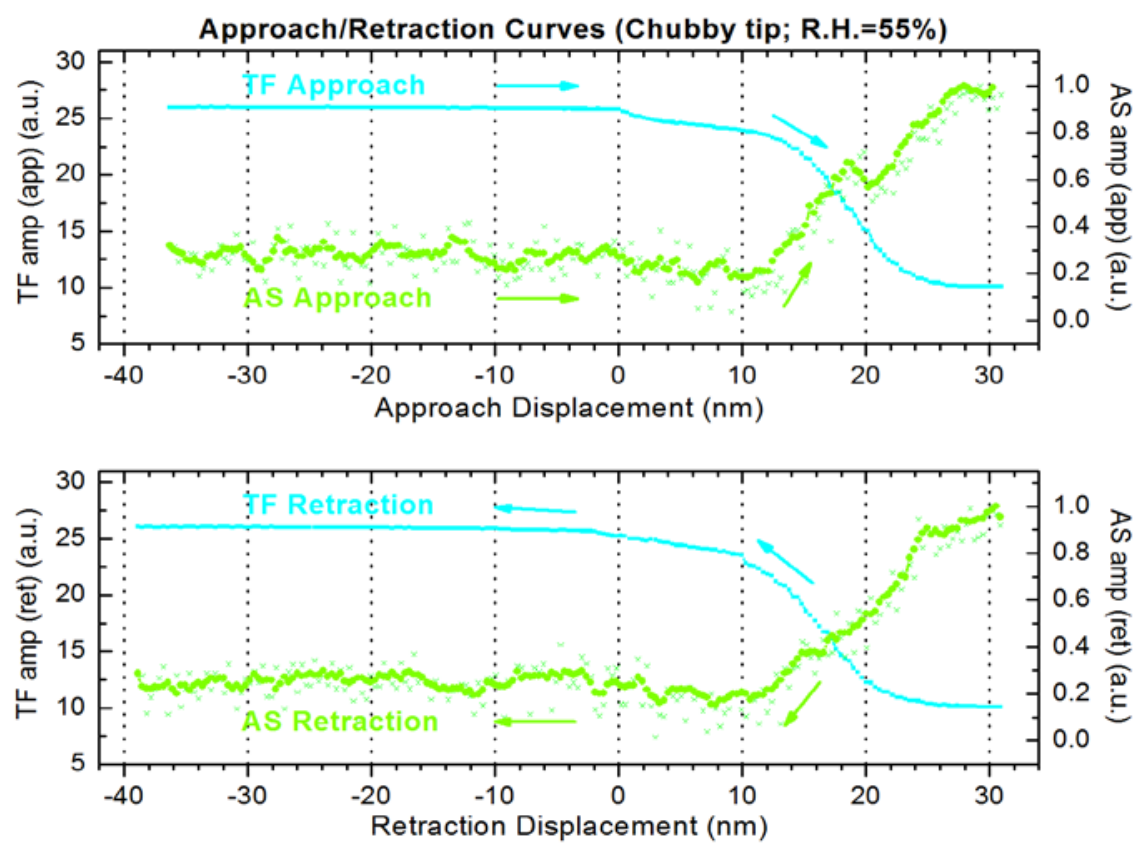

Figure 5.7: Approach (upper) and retraction (lower) curves of TF (light blue) and acoustic (light green) signals obtained with the use of a chubby tip and a bare silicon wafer (humidity: 55\%).
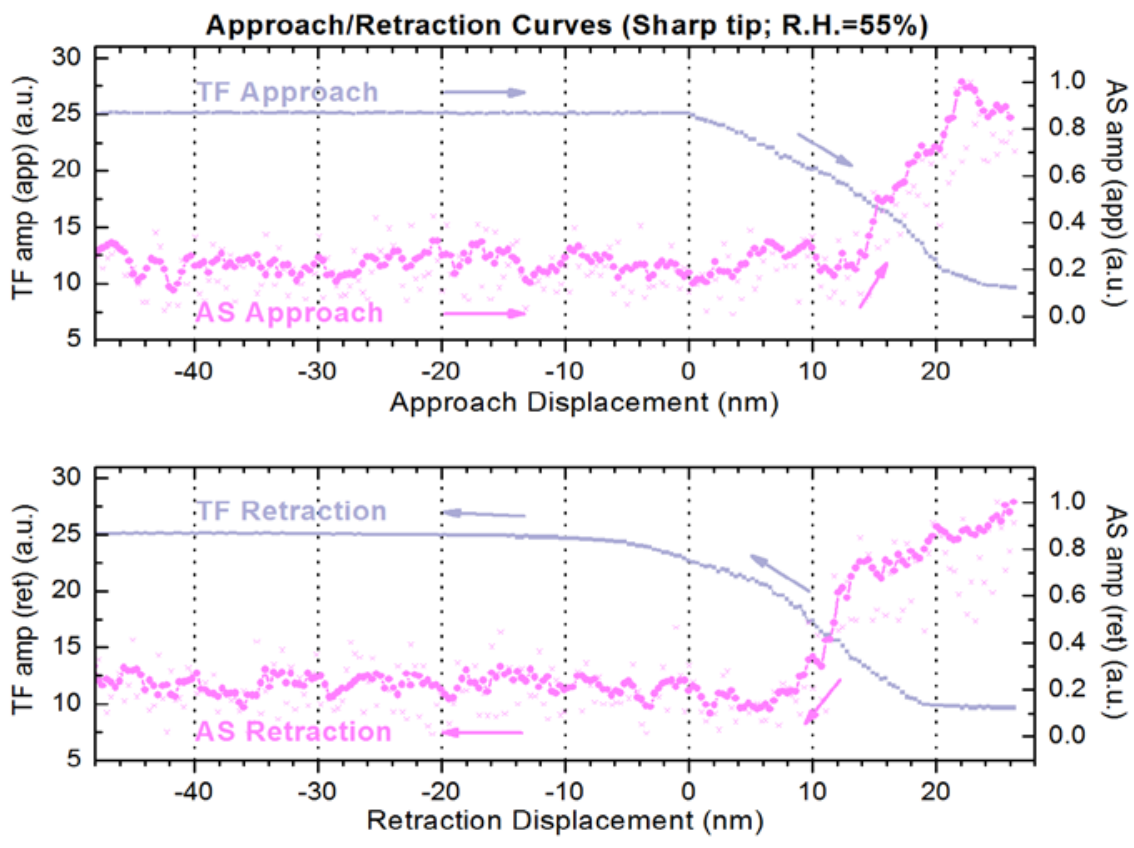

Figure 5.8: Approach (up) and retraction (lower) curves of TF (light purple) and acoustic (light pink) signals obtained with the use of a sharp tip and a bare silicon wafer (humidity: $55 \%)$. 
We re-drew the results obtained at R.H.= 25\% and R.H. $=55 \%$ using different colors in the same figures for convenience. For the chubby tip, comparison of the TF and acoustic signals at different humidity is demonstrated in Figures 5.9 (approach curves) and 5.10 (retraction curves). The most significant distinction between the curves recorded under low R.H and high R.H. conditions is the length of the transition between the maximum and the minimum of the measured signals: the transition portion elongates at high R.H.. This can be explained by the growth of the humidity-dependent contamination layer, which is composed of water molecules absorbed from the atmosphere to the solid surface: the higher the humidity the more water molecules are expected to be trapped on the sample. Evidence to support our viewpoint is the presence of a slow decay region on the TF approach curve at high R.H. (55\%). By contract, it is not evident at low R.H. (25\%). The appearance of the slow decay region is in agreement with the results in literatures where similar experiments have been carried out ${ }^{72,74,76}$. A similar extension observed on the acoustic curves can be considered as another confirmation of our assumption that the contamination layer grows when raising the humidity. For the sharp tip, we plotted the experimental results in Figures 5.11 (approach curves) and 5.12 (retraction curves). The elongation of the transition region on the approach and retraction curves at high R.H is consistent with our observation on the chubby tip, suggesting that the contamination layer becomes thicker as R.H increases. 

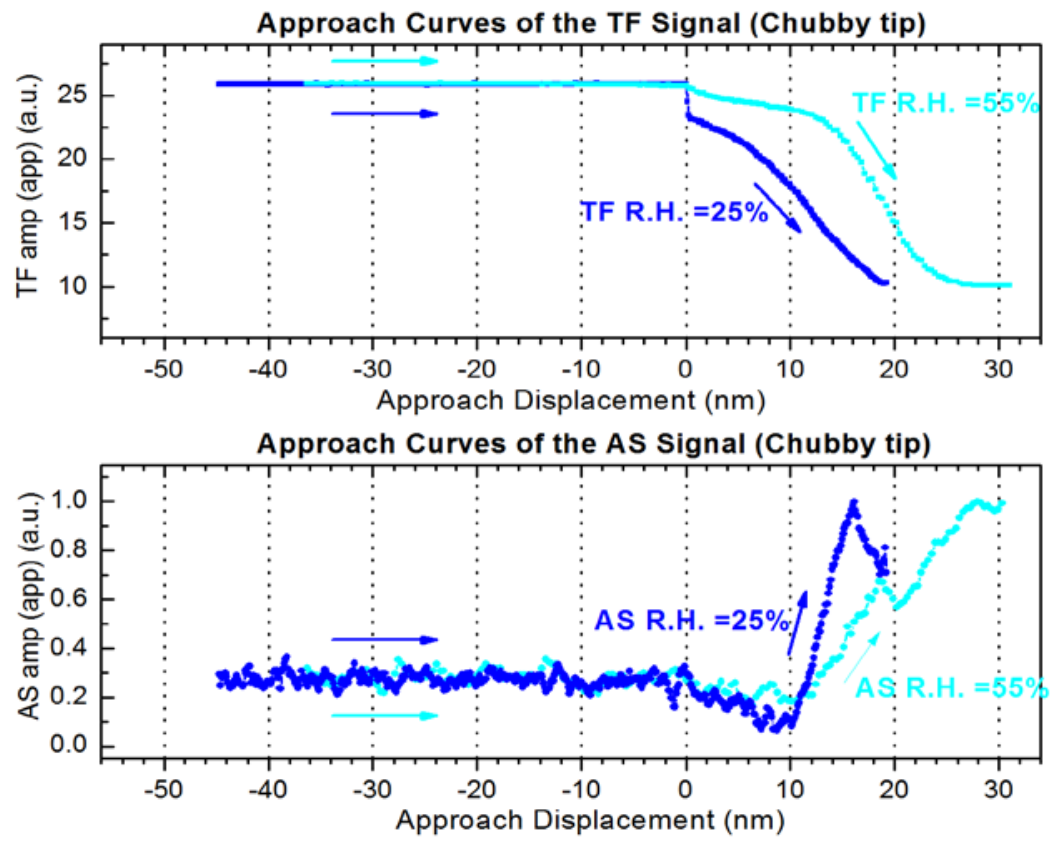

Figure 5.9: Approach curves of TF (upper) and acoustic (lower) signals obtained using the chubby tip at R.H. $=25 \%$ (blue) and R.H. $=55 \%$ (light blue).
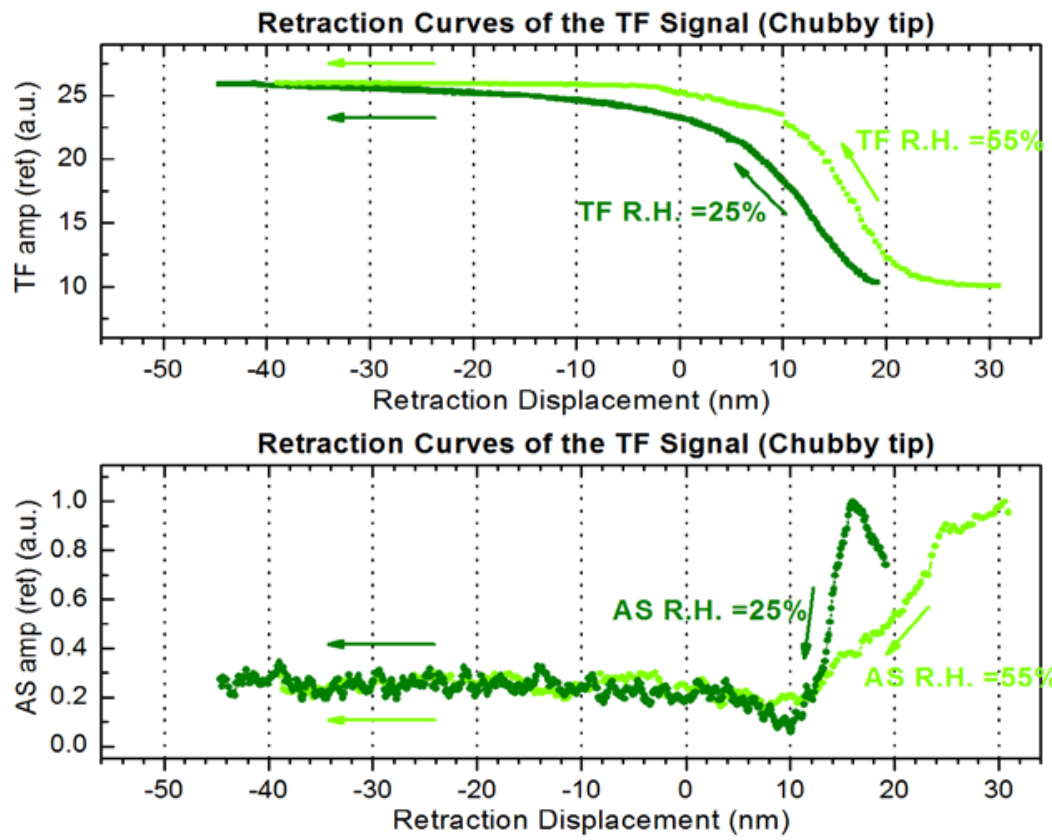

Figure 5.10: Retraction curves of TF (upper) and acoustic (lower) signals obtained using the chubby tip at R.H.=25\% (green) and R.H.=55\% (light green). 

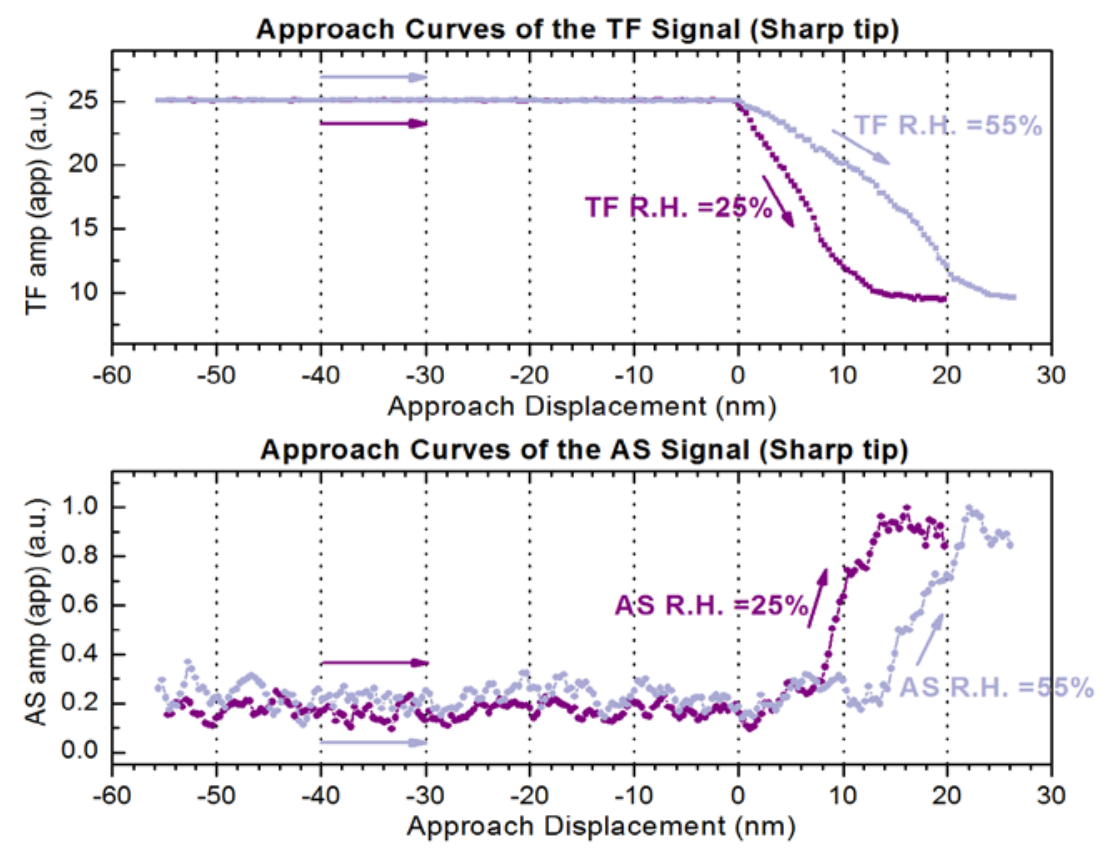

Figure 5.11: Approach curves of TF (upper) and acoustic (lower) signals obtained using the sharp tip at R.H.=25\% (purple) and R.H.=55\% (light purple).
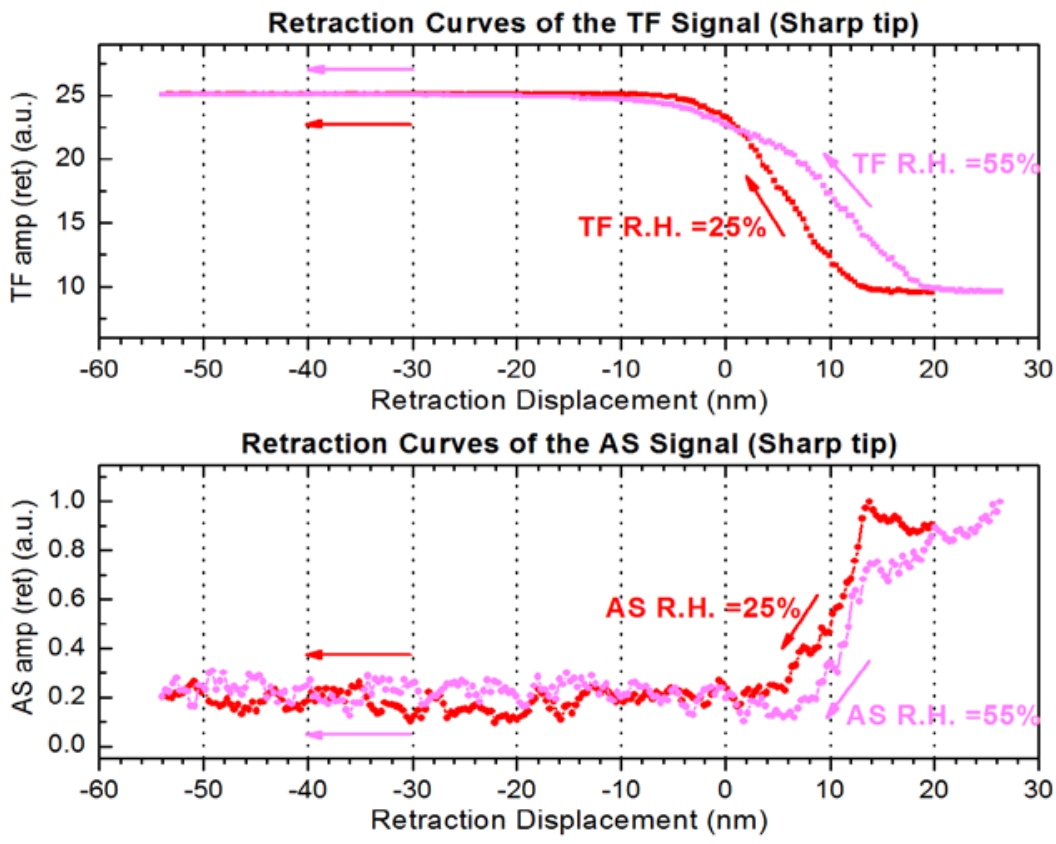

Figure 5.12: Retraction curves of TF (upper) and acoustic (lower) signals obtained using the sharp tip at R.H.=25\% (red) and R.H.=55\% (light pink). 
We notice that, in Figure 5.10, the retraction curve obtained at low R.H. shows a long tail. It is probably due to the presence of mobile charges on the tip and the surrounding. The electrostatic interaction between the probe and the sample may become more notable at low R.H. for the chubby tip. Therefore, the tip has to undergo a longer retraction distance until it fully returns to its initial oscillation amplitude.

\subsubsection{Effect of Properties of Sample Surfaces}

\section{A. Silicon Substrates Cleaned Using Different Methods}

As a measurement of the probe-sample interaction, the shear-force and acoustic signals are sensitive not only to the properties of tips but also to the natures of sample surfaces. Silicon wafers are the most commonly used substrates in our experiments. The purity of silicon is an essential factor which will affect the experimental results.

Therefore, small pieces of silicon wafers must be cleaned prior to use. The cleaning methods are manifold, depending on the specific requirements of experiments. For example, particulate impurities can be successfully removed by ultrasonic treatment in detergent solutions. Piranha solution can be used to digest the organic component of impurities $^{77}$. An immersion in aqueous HF solution is sufficient to remove the silicon oxide film which may trap impurities ${ }^{78}$. Alternatively, dry-cleaning techniques (i.e., $\mathrm{O}_{2}$ plasma etching) are powerful for removing many contaminants.

Herein, our aim is to explore the effect of different cleaning techniques in shearforce/acoustic measurements, thereby developing a standard cleaning procedure in sample preparation. Cleaning of silicon wafers was respectively carried out using Piranha 
solution, $\mathrm{HF}$ etching, and $\mathrm{O}_{2}$ plasma etching for comparison purpose. After sample cleaning, approach/retraction curves of the TF and acoustic signals were simultaneously acquired by moving a gold tip close to and away from the cleaned surface at a constant velocity of $2 \mathrm{~nm} / \mathrm{s}$. The same tip was used in the whole experiment (obtaining approach/retraction curves on silicon samples cleaned using different methods); it was rinsed with distilled water before and after the acquisition of each pair of curves. In order to avoid tip contacting with and crashing into the hard surface, approach process stops once the tip’s amplitude reaches $60 \%$ of its maximum, and tip retraction is immediately preformed. The "zero" coordinates of the horizontal axis was arbitrarily selected at the point where the amplitude of the TF signal drops to $95 \%$ of the initial value during the approach process.

Piranha solution is a mixture of sulfuric acid and hydrogen peroxide. It has been used in the semiconductor industry for many years as the primary method of removing organics like photoresist. Mixing hydrogen peroxide $\left(\mathrm{H}_{2} \mathrm{O}_{2}\right)$ and sulfuric acid $\left(\mathrm{H}_{2} \mathrm{SO}_{4}\right.$, $>95$ wt\% ) forms Caro’s acid, which is the active etchant in a Piranha bath ${ }^{77}$. In our experiment, Piranha solution composed of $35 \% \mathrm{H}_{2} \mathrm{O}_{2}$ and $98 \% \mathrm{H}_{2} \mathrm{SO}_{4}$ in the ratio of 3:7 by volume was freshly prepared prior to use. Silicon wafers were first immersed in such Piranha solution, stirring for $60 \mathrm{~min}$ at $80-90{ }^{\circ} \mathrm{C}$. They were then washed with boiling water for $90 \mathrm{~min}$ and dried under $\mathrm{N}_{2}$. Figure 5.13 shows the approach/retraction curves obtained right after the Piranha treatment (Figure 5.13a) and 24 hours after the cleaning (Figure 5.13b). 
(a)

Right After Piranha Cleaning

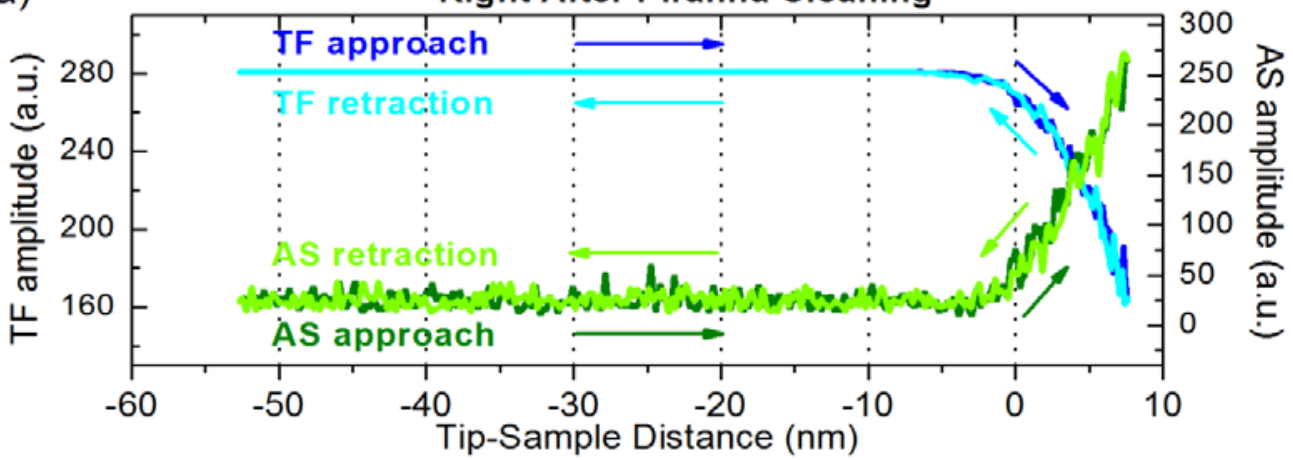

(b)

24h After Piranha Cleaning

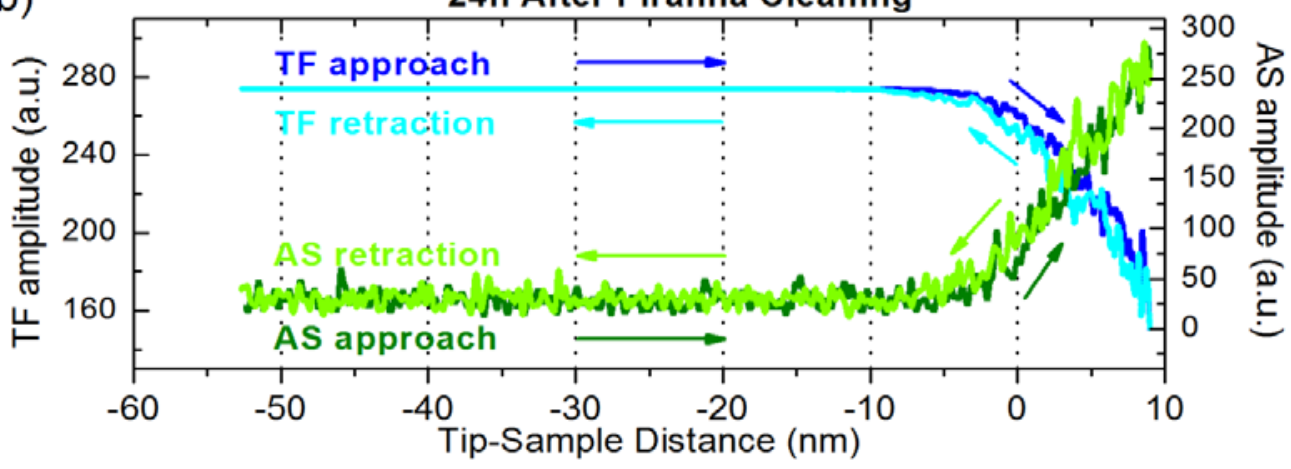

Figure 5.13: Approach/retraction curves of the TF and acoustic signals on silicon substrate cleaned using Piranha solution: (a) curves obtained immediately after the Piranha treatment; (b) curves obtained on the same surface, 24h after the cleaning.

Silicon is the mostly used semiconductor material in the manufacture of integrated circuits. Accordingly, the surface of silicon wafers is of essential importance in deciding the growth and stability of functional structures on it. A great deal of research work related to the surface chemistry of silicon has been experimentally carried out on clean surfaces that undergo complex reconstructions in ultrahigh vacuum (UHV) chambers ${ }^{79-81}$. Alternatively, silicon surfaces can be passivated by terminating the surface dangling bonds ${ }^{82}$ through various treatments, which cannot only restore contamination-free surface in ambient conditions but also modify the nature of the surface ${ }^{78,83,84}$. Passivation of silicon surfaces by hydrofluoric acid (HF) has been used as a powerful method to remove 
the oxide layer and keep the surface clean. It was initially suggested that fluorine termination of the surface dangling bonds was responsible for the resulting near perfect surface stability ${ }^{85}$. However, it has become understood that the great stability of these surfaces is actually attributed to surface passivation by hydrogen ${ }^{78,86-88}$ : a monolayer of hydrogen, instead of fluorine, is present at the surface to protect the surface from chemical attack. Hydrogen terminated (H-terminated) silicon surfaces have received increasing attention and been often used as the starting substrate for further processing of novel modified silicon surfaces ${ }^{89-91}$. The chemical and physical properties of $\mathrm{H}-$ terminated silicon surfaces are reasonably stable so that they can be prepared and manipulated in air before measurable oxidation of the surface occurs. Therefore high quality surfaces are available without the need for expensive vacuum systems. Hydrogen termination of the Si(111) surface can be simply achieved by chemical etching in aqueous $\mathrm{HF}$ acid, whereas high quality $\mathrm{Si}(100)-\mathrm{H}$ surfaces are produced by exposing the clean surface to hydrogen atoms under UHV conditions. In our experiment, Si(111) substrates were made H-terminated by immersing it in a dilute $\mathrm{HF}$ solution $\left(1.5 \%\right.$ in $\left.\mathrm{H}_{2} \mathrm{O}\right)$ for 15 min at room temperature following Piranha cleaning described above. The Piranha treatment is optional. Our purpose here is to demonstrate the change in hydrophilichydrophobic nature of the silicon surface from the Piranha-treated wafer (hydrophilic) to the HF-etched sample (hydrophobic). As shown in Figure 5.14, by performing contact angle measurements on the resulting surfaces, it is evident that the hydrophilic oxidized surface obtained after Piranha cleaning is converted to hydrophobic by performing HF etching, which is thought to be explained by the formation of hydrogen termination. 
Immediately after the HF treatment, silicon substrates were rinsed with distilled water and dried under $\mathrm{N}_{2}$, then placed on the acoustic sensor for shear-force/acoustic measurements. Figure 5.15 shows the approach/retraction curves obtained immediately after the HF treatment (Figure 5.15a) and 24 hours after the etching (Figure 5.15b).

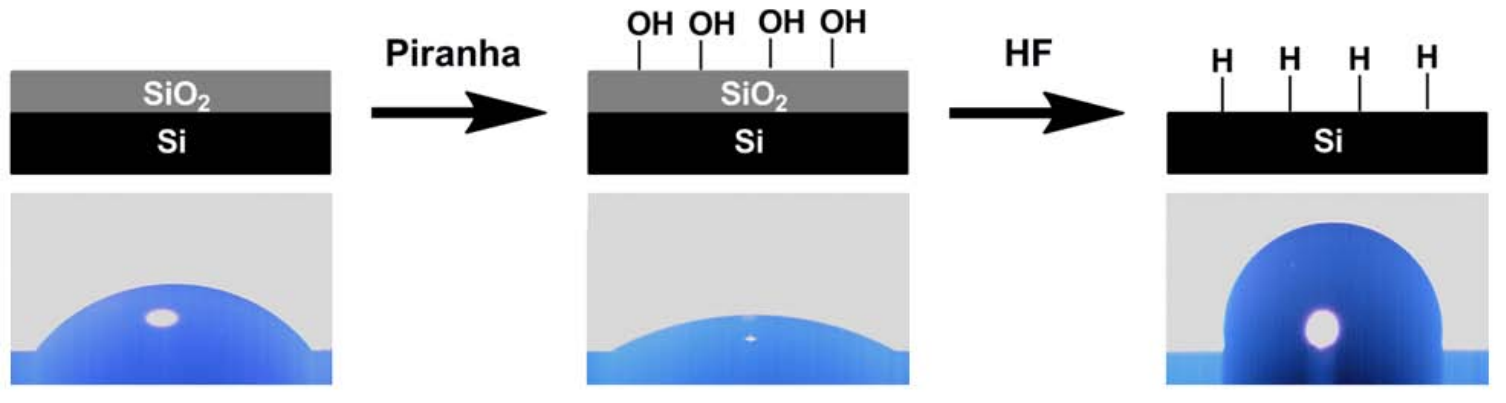

Figure 5.14: Scheme of experimental procedure to prepare H-terminated Si surfaces. Contact angle measurements on the corresponding surfaces (lower) show the change in hydrophobic-hydrophilic nature of the surface. 
(a)

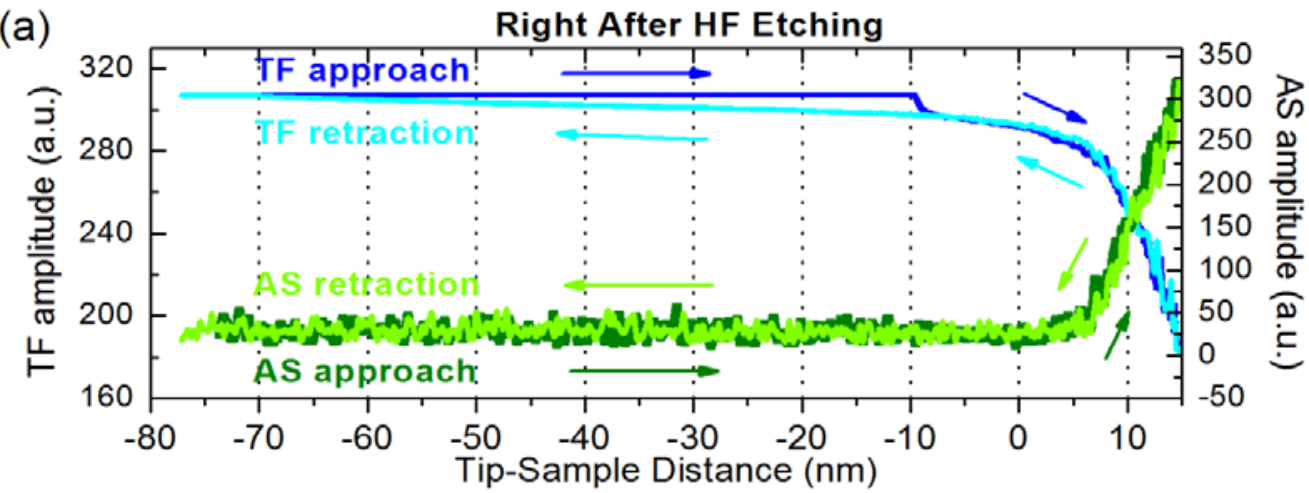

(b)

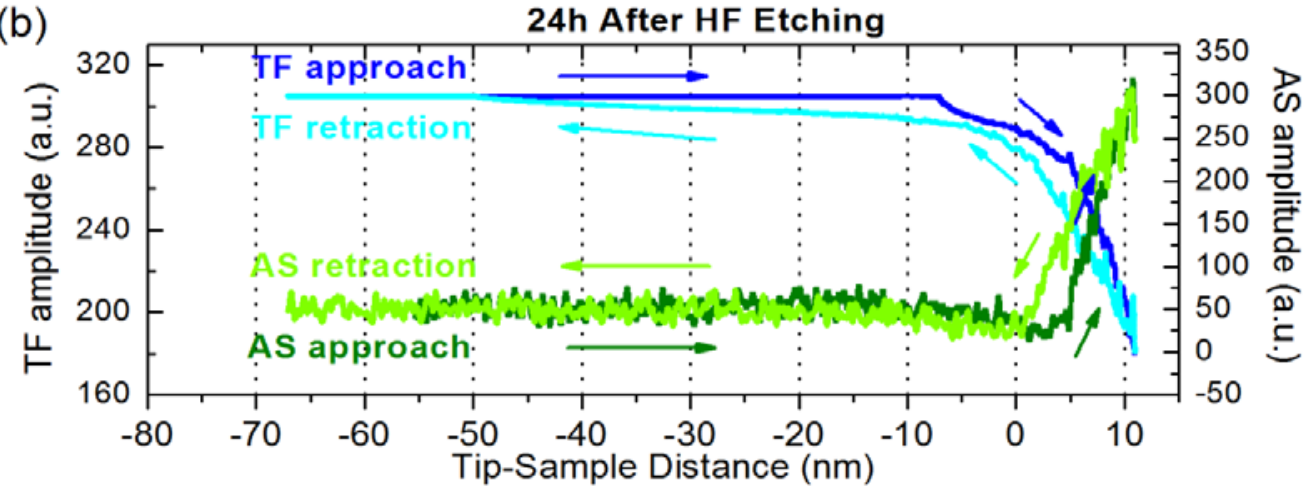

Figure 5.15: Approach/retraction curves of the TF and acoustic signals on silicon substrate treated by aqueous HF solution: (a) curves obtained immediately after the HF treatment; (b) curves obtained on the same surface, 24h after the etching.

Plasma etching, as one of the most used dry etching techniques in the fabrication of integrated circuits and microsystems, replies on radio frequency (RF) power to drive chemical reactions. The generation of plasma is categorized as: capacitively coupled RF plasmas, inductively coupled RF plasmas and Electron Cyclotron Resonance plasmas. Compared with other methods, plasma etching has several advantages, such as less usage of toxic chemicals and solvents, highly directional etching, high-resolution/selectivity, better process control and ease of automation. The working process involves a fast-speed stream of glow discharge plasma gas species (either charged ions or neutral radicals/atoms) that can be generated and transferred to target semiconductor surface. 
New molecules are formed when the gas mixture interacts with solid atoms removed from the substrate ${ }^{92,93}$. For example, when reactive gas oxygen $\left(\mathrm{O}_{2}\right)$ is used as plasma source, the discharges remove the organic contaminants and subsequently leave an oxide residue layer ${ }^{94}$. In our experiment, $\mathrm{Si}(111)$ wafers were carefully placed into the small vacuum reactor to perform $\mathrm{O}_{2}$ plasma etching, and the total etching time was $15 \mathrm{~min}$. Figure 5.16 shows the approach/retraction curves obtained right after the $\mathrm{O} 2$ plasma etching (Figure 5.16a) and 24 hours after this treatment (Figure 5.16b).
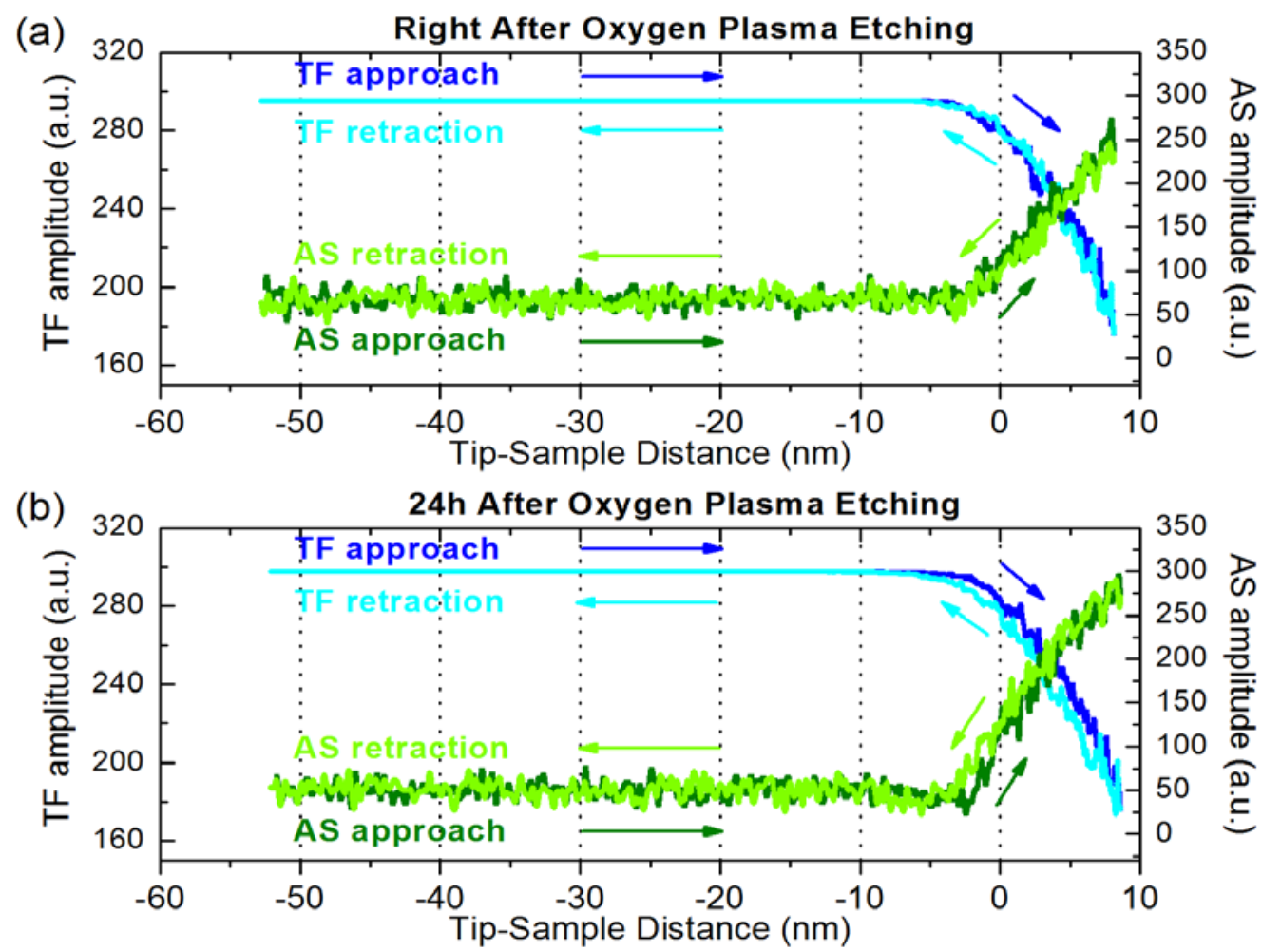

Figure 5.16: Approach/retraction curves of the TF and acoustic signals on silicon substrate cleaned with $\mathrm{O}_{2}$ plasma: (a) curves obtained immediately after the plasma etching; (b) curves obtained on the same surface, 24h after the plasma etching.

First of all, comparison was made among the resulting approach/retraction curves obtained immediately after surface cleaning using different methods. One compelling 
characteristic is that, all graphs show negligible hysteresis between the approach and the retraction curves. Hysteresis may incorporate information regarding the surface in response to the tip's behavior. However, such information could be disturbed and hidden by impurities either on the surface or in the liquid film. According to our experimental observation, the mesoscopic fluid layer starts to form immediately after cleaning on the wafer as soon as it is exposed to the ambient atmosphere, leading to the attenuation of the TF signal and the generation of acoustic waves. Our results show that, by removing undesired contaminants from a silicon surface, hysteresis from the fluid film is much reduced (even avoided). A cleaning procedure during sample preparation provides an opportunity to attain a reference substance. On a reference sample, there is a lack of hysteresis between the approach and the retraction curves, which is different from our common knowledge on the sample that is not cleaned. We can take advantage of this property of the reference sample to study the effect of adhesion between the tip and the hard surface when they are in contact with each other. Since hysteresis resulted from the absorbed fluid layer has been avoided, hysteresis shown on the resulting curves will be attributed to the solid-solid contact. Furthermore, after performing surface modification on the reference surface, hysteresis may return as a result of the purposeful tailor. With the use of reference substrates, it is promising to use the shear-force/acoustic microscope as a proper tool to characterize solid surfaces.

Secondly, we compare the curves obtained immediately after cleaning and 24 hours after cleaning. After 24 hours, hysteresis becomes visible on both the TF and acoustic curves. We can rule out hysteresis caused by the detection system (lock-in amplifiers) 
without any hesitation because the settings of lock-in amplifiers were not changed at all. Therefore, the appearance of hysteresis after 24 hours may reveal a transition taking place in the liquid layer or/and on the sample surface. The freshly cleaned sample attracts water; and over time it can also absorb oil and any other vapor that is present in the laboratory. Thus, those impurities absorbed during a long period (i.e., 24 hours) will continuously contaminate the liquid layer on the surface, which likely contributes to the recurrence of hysteresis. On the other hand, we cannot rule out the possibility that hysteresis is caused by the change on the silicon surface. For example, it is known that the H-terminated silicon surface can be slowly oxidized when exposed to air for a long term. The presence of moisture in humid air can lead to the growth of oxide layer with time on the surface by conversion of surface Si-H bonds to oxygen-related bonds, such as $\mathrm{Si}-\mathrm{OH}$ and $\mathrm{Si}-\mathrm{O}-\mathrm{Si}$ bonds ${ }^{95,96}$. This change on the solid surface can also affect the structure of the liquid film, leading to detectable hysteresis on resulting curves. By contrast, on the Piranha treated silicon surface, which is more hydrophilic due to the induced OH groups ${ }^{97,98}$ (Figure 5.14), we do not expect changes like the transition from $\mathrm{Si}-\mathrm{H}$ to $\mathrm{Si}-\mathrm{OH}$ on the surface. Therefore, the appearance of hysteresis is probably owing to the mesoscopic liquid layer, for example, changes in the composition of the layer over time. Altogether, the measured approach/retraction curves vary with the freshness and cleanliness of the sample and the tip. A careful cleaning procedure is a crucial step during sample preparation. 


\section{B. Bare Si Surfaces vs. Polymer-coated Si Surfaces}

As a first step in experiments we modified a bare silicon wafer by covering a thin polymer film on its surface. We attempt to compare the approach/retraction curves obtained on the bare silicon with those acquired on the polymer coated surface. Prior to use, the silicon wafer was cleaned using acetone with ultrasonic agitation for at least 30 min and dried by blowing $\mathrm{N}_{2}$. A poly(4-vinylpyridine) (P4VP) film (the thickness of $\sim 20$ $\mathrm{nm}$ ) was spin coated on the silicon substrate. Immobilization of this polymer film was achieved by subsequent exposure to UV light ${ }^{99}$. The polymer sample can be ultrasonically cleaned using IPA for 1 3 min if needed. Our experiment was carried out under moderate humidity condition (R.H. was 30\%). The same experimental procedure using the same tip was run on the bare silicon wafer and the polymer coated silicon substrate, respectively: the approach process stopped when the amplitude of the tip's vibration fell to $60 \%$ of its initial value, and subsequently, the retraction performance started without any time delay. The speed of the tip’s movement remained constant at 2 $\mathrm{nm} / \mathrm{s}$. 

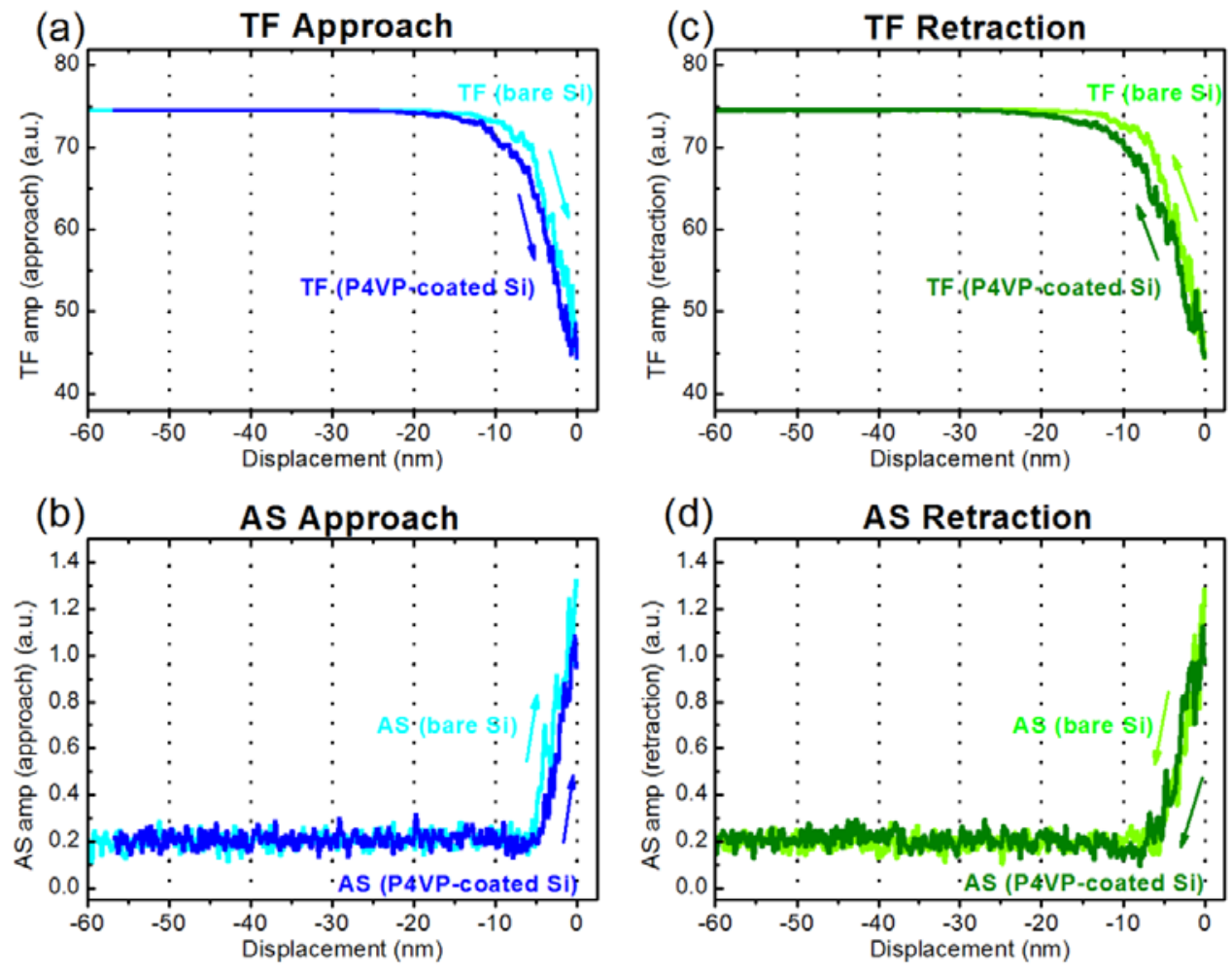

Figure 5.17: Approach (a and b) and retraction (c and d) curves of TF and acoustic signals obtained on bare silicon (light blue and light green) and polymer coated silicon (blue and green) substrates.

Comparison was made between the result obtained on the silicon surface and that obtained on the polymer surface. As shown in Figure 5.17, with the use of the same gold tip, the curves obtained on the polymer covered substrate are quite similar to those acquired on the bare silicon surface. The origin of the horizontal axis represents the position where the approach process stops or the retraction process begins. We assume when the approach process stops, the tip stays in the fluid layer without touching the sample surface. It seems that the shape of the measured curve does not vary too much with the change of testing surfaces. Since the bare silicon surface and the polymer coated surface were not prepared on the same substrate, the slight difference in the amplitude of 
the acoustic signal shown in Figures 5.17b and 5.17d may be due to the difference in the acoustic coupling efficiency between the sample and the sensor.

\section{Si-based Substrates with Modified Water Affinity}

In a second step of surface modification, we tailored the water affinity of the silicon substrate by depositing specific polymer monolayers ${ }^{99,100}$. Firstly, a bare silicon wafer cleaned with Piranha solution was chemically functionalized with perfluorophenylazides (PFPA)-silane to introduce a monolayer of azido groups on the substrate surface.

Secondly, a $10 \mathrm{mg} / \mathrm{ml}$ solution of polystyrene (PS) in toluene was spin coated on the PFPA-tethered water. The PS-coated substrate was subsequently irradiated under a 450 W medium-pressure mercury lamp with $280 \mathrm{~nm}$ filter for 5-10 min. Half the surface of the sample was covered by aluminum foil, and only the half without aluminum foil coverage was exposed to the UV irradiation. After removal of unbound PS by solvent extraction, a PS thin film ( 6 nm thick) covered half the wafer. Finally, the PS solution was replaced by a $10 \mathrm{mg} / \mathrm{ml}$ solution of poly(2-ethyl-2-oxazoline) (PEOX) in chloroform; and the same procedure described above was repeated for coating a PEOX thin film which approximately had the same thickness as that of the PS film on the inactivated substrate surface. Figure 5.18 demonstrates the entire procedure of chemical treatment to create the PS/PEOX coated surface. 


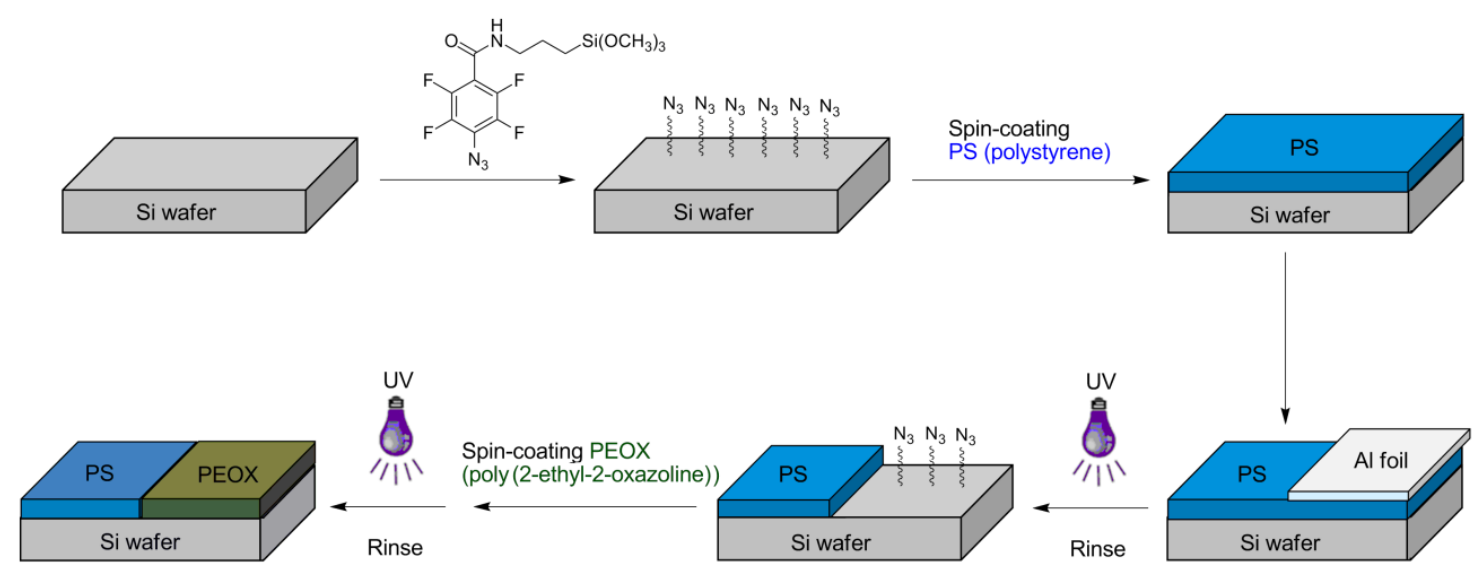

Figure 5.18: Scheme of experimental procedure for preparing hydrophobic and hydrophilic surfaces on the same Si substrate.

Water affinity of the sample surface coated with specific polymer thin films can be characterized by performing contact angle measurements. Results displayed in Figure 5.19 show that the side covered with PS monolayer is hydrophobic (contact angle is about $89^{\circ}$ ), whereas the other side coated with PEOX monolayer is hydrophilic (contact angle is about $36^{\circ}$ ).
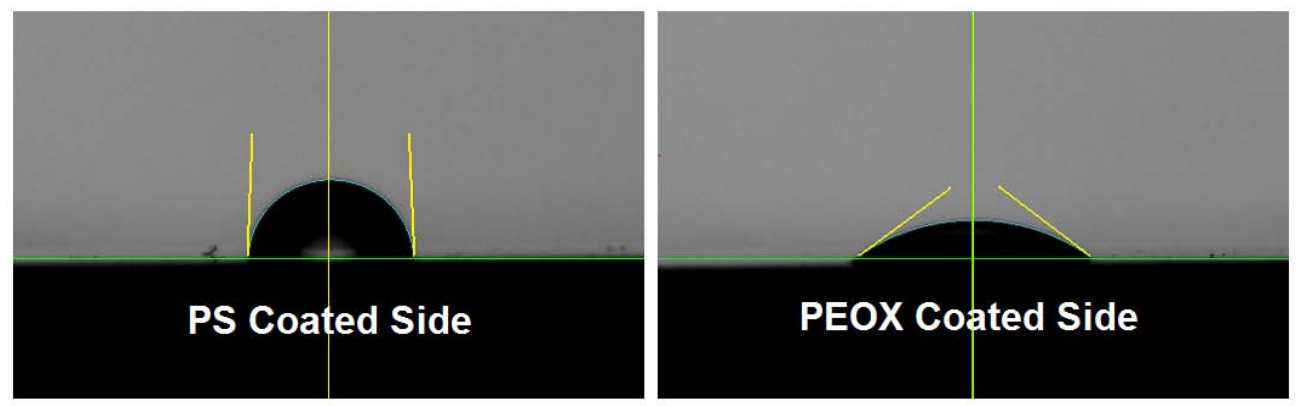

Figure 5.19: Results of contact angle measurements on the same Si substrate covered by PS and PEOX monolayer on each half, respectively.

A sharpened gold tip attached to one prong of a TF was used as the probe. The TF was electrically driven to oscillate at its resonance frequency by applying a voltage of 10 
$\mathrm{mV}_{\text {rms }}$. The estimated amplitude of the probe's oscillation in air is about $16 \mathrm{~nm}$.

Variations of the TF and acoustic signals were recorded as a function of the tip-sample distance while the tip moved in the vertical direction at a constant velocity of $1.5 \mathrm{~nm} / \mathrm{s}$. Figures 5.20 and 5.21 respectively illustrate the approach/retraction curves obtained on the PS coated (hydrophobic) and the PEOX coated (hydrophilic) sides using the same gold tip. The experiment was carried out in an environment with moderate humidity (R.H.= 35\% 40\%).

In these figures, the resulting approach/retraction curves exhibit similarities to those obtained in previous experiments. For instance, as to the curves in Figure 5.20, the tip initially vibrates at its resonance in air, which also produces sound waves as a nonzero background of the acoustic measurement (region I). Subsequently, the tip is brought close to the sample; its vibration remains unchanged until it enters the contamination layer in which a decrease in the TF amplitude is accompanied with an increase in the acoustic signal as a result of the probe-sample interaction. We arbitrarily define the location where the TF signal starts to fall as the origin of the horizontal axis. With the ongoing approach process, the tip penetrates deeper into the liquid and moves closer to the solid surface. We artificially divide the rest of the approach curve into two regions (region II and III in Figure 5.20a). In region II, the amplitude of the tip’s vibration suffers from a substantial attenuation, whereas the amplitude of the measured acoustic signal significantly increases above the background level. We call the TF and the acoustic signals are negatively correlated. In the following region III, the TF amplitude consistently decreases, similarly, the acoustic amplitude also decreases; and so the correlation between the TF and the 
acoustic signals switches to positive. A plausible hypothesis to explain our observation is to consider a distance-dependent interaction between the tip and the liquid proposed previously $^{73}$. In region II, as the probe-sample distance is getting shorter, increasing phase lagging between the vibrating tip and the mesoscopic fluid, even the "solidification" of the fluid-like layer upon confinement may occur ${ }^{101}$, leads to significant changes in the TF and acoustic signals following opposite tends. At the end of the approach (region III), it is possible that mechanical contact of the probe apex with the polymer film takes place, which is considered to be responsible to the dramatic decrease in these two signals. The same regions can be correspondingly found on retraction curves. However, the tip undergoes a longer traveling distance until it returns to its initial oscillation amplitude. Hysteresis, indicative of the extension of the retraction curve compared with the approach curve, probably originates from the surface adhesion after the tip contacts the polymer layer as well as the presence of the contamination layer. A similar analysis can also be applied to the curves in Figure 5.21, which were obtained on the PEOX coated silicon substrate. 

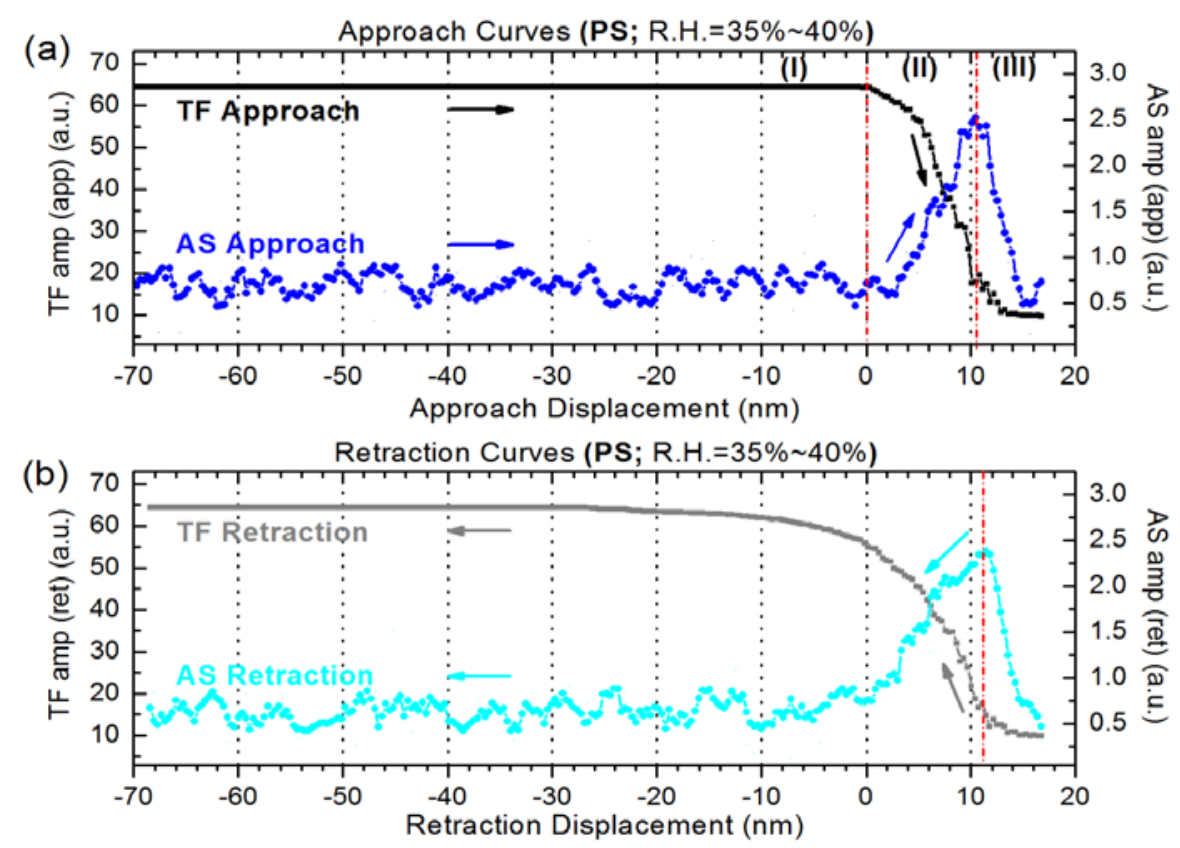

Figure 5.20: Approach (a) and retraction (b) curves of TF (black and gray) and acoustic (blue and light blue) signals obtained on the PS film coated side with the use of a gold tip (humidity: 35\% 40\%).
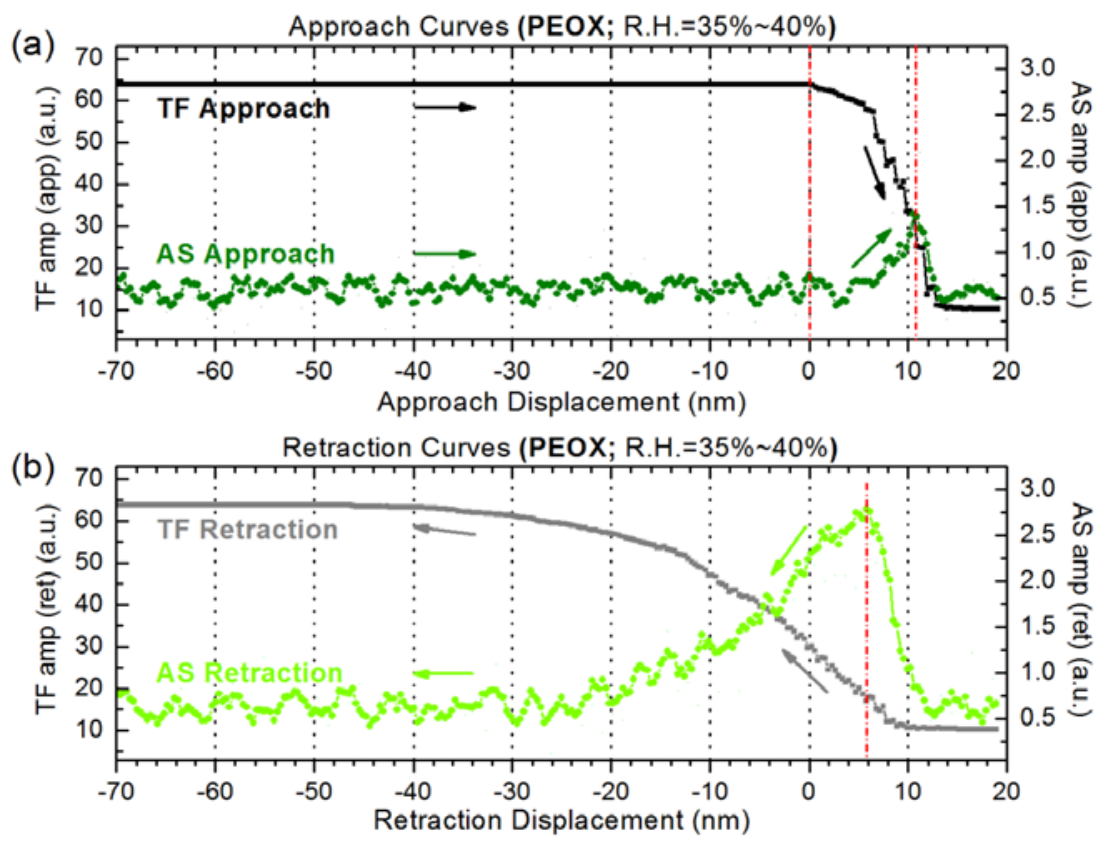

Figure 5.21: Approach (a) and retraction (b) curves of TF (black and gray) and acoustic (green and light green) signals obtained on the PEOX layer covered side by employing the same gold tip as used on the PS coated side (humidity: 35\% 40\%). 
In fact, we are still not able to fully confirm the effect of the mechanical contact between the tip and the polymer surface through shear-force/acoustic measurements. For example, the tapping of the tip on the sample may also happen in region II (Figure 5.20a), contributing to the fast change in signals. Therefore, other probing technique (i.e., detection of the probe-sample current) will be worth integrating into our shearforce/acoustic microscope to further explore the solid-solid (probe-sample) interaction on the bottom of the mesoscopic liquid layer, in proximity to the hard surface. Despite that monitoring of contact current is not feasible on polymer coated surfaces, it becomes the motivation of work presented in the following chapter. As a first attempt, analogous experiments performed on gold surfaces using gold tip will be helpful to gain a better understanding of information extracted from the approach/retraction curves of the TF and acoustic signals.

For comparison purpose, we re-drew our experimental results in Figure 5.22. We observed that the tip travels slightly longer distance for the transition from the maximum vibration amplitude to the minimum amplitude to occur on the PEOX coated substrate than that on the PS coated wafer (Figures 5.22a and 5.22b, comparison between approach traces obtained on the PS and the PEOX surface). Comparison of their retraction traces exhibits this difference between the PS coated side and the PEOX coated side more obviously (Figures 5.22c and 5.22d). Hysteresis given by the PEOX (hydrophilic) coating is also more noticeable than that observed on the PS (hydrophobic) surface. In our previous studies on cleaned surface, there was a lack of hysteresis on the resulting curves if the experiment was performed immediately after sample cleaning (i.e., Figure 5.13a). It 
is because, in those experiments, we intended avoiding contact of the tip with the surface by stopping approach when the tip was still in the middle of the fluid layer. Herein the situation is different: we forced the tip to penetrate deeper into the liquid, and hysteresis is present as a result of contact between the tip and the polymer.

More interesting, we found that, when placing the tip on the PEOX coated surface, the maximum of the acoustic signal on the approach curve is much less than that on the retraction curve; whereas the two maxima of the acoustic signal are almost the same when performing the same experiment on the PS coated surface (Figure 5.22e). To elucidate this observation, we propose one hypothesis that the water flowing over the surface contributes to the sound generation. In the region very near the sample surface, with the continuous approach of the tip to the surface, the liquid is extruded sidewards by the tip. However, the flow of water, which is a potential mechanism for producing sound, is unfavorable on the hydrophilic surface because of the strong interaction between polymer and water molecules. Hence less sound is produced during approach on the PEOX coated surface. In contrast, once the tip is released from the hydrophilic surface, the liquid rapidly refills in for returning to its original arrangement, causing a high peak on the measured acoustic curve during the retraction process. By contrast, on the PS coated surface, "binding" between water and polymer molecules is weak because the coating substance is "water-hating", and therefore restriction to the flow of water becomes less on such surface. If we still consider the flow of water makes efforts to generate sound waves, peaks on the acoustic curves due to movement of water molecules have similar height when moving the tip to approach and retract at the same speed. It may 
imply that the behavior of water films is dominated by the tip's movement on the hydrophobic surface. 

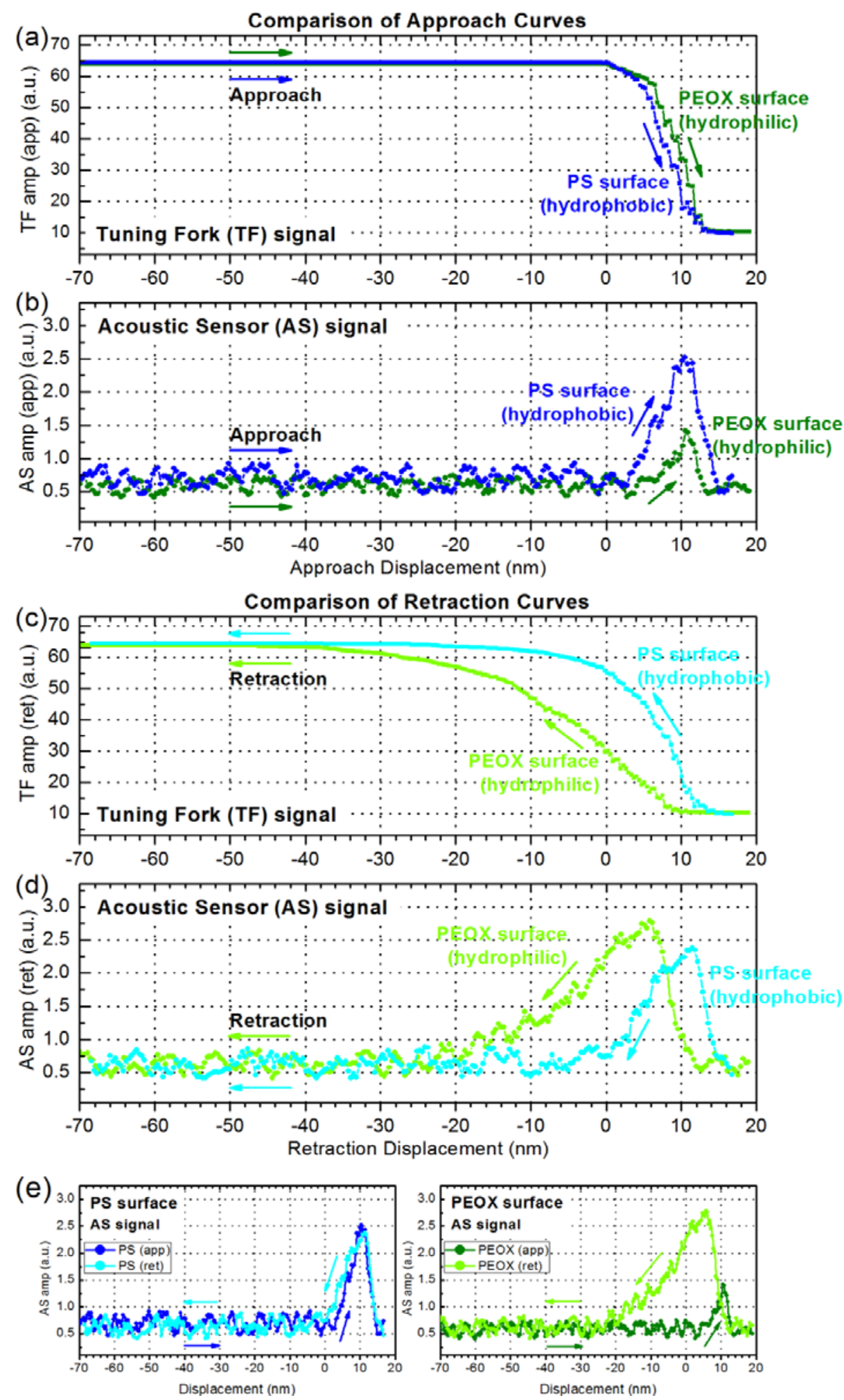

Figure 5.22: Approach curves of the TF (a) and acoustic (b) signals obtained on the PScoated (blue) and PEOX-coated (green) silicon wafer; and retraction curves of the TF (c) 
and acoustic (d) signals obtained on the PS-coated (light blue) and PEOX-coated (light green) silicon wafer. The experiment was performed by using the same tip at R.H. = 35\% 40\%. (e) The acoustic approach/retraction curves were re-drawn by separating the PS surface from the PEOX surface.

\subsubsection{Effect of Velocity of Probe Motion}

In previous experiments, the tip moved perpendicular to the sample surface at a constant speed which was kept the same in both approach and retraction processes. In this section, the effect of velocity of tip motion on the resulting curves is discussed. A gold surface was used as the sample. A sharpened gold tip acted as the probe as usual. In general, it is difficult to estimate the actual position of the solid surface via measuring the shear-force signal. However, by applying a bias voltage across the conductive surface (gold) and the metal tip (gold), we can approximately locate the hard surface through monitoring the probe-sample current, which indicates a mechanical contact between them. Details regarding detection of the current signal (i.e., experimental setting, procedure, resulting approach/retraction curves and corresponding analysis) will be given in the following chapter. Herein we aim to bring the tip as close as possible to the "true" surface. Measurement of the contact current is a valid way to help us judge the location of the solid surface. We expect that, the tip will penetrate the whole absorbed film without destroying the apex when the tip’s approach is stopped right before the appearance of current pulses. After completing the approach process, the retraction process is immediately carried out without time delay. Electrical signals of the TF and the acoustic sensor were concurrently recorded with changing the probe-sample distance and plotted 
as the approach/retraction curves. Velocity of tip motion is variable so that we are able to test the impact of velocity on the obtained curves.
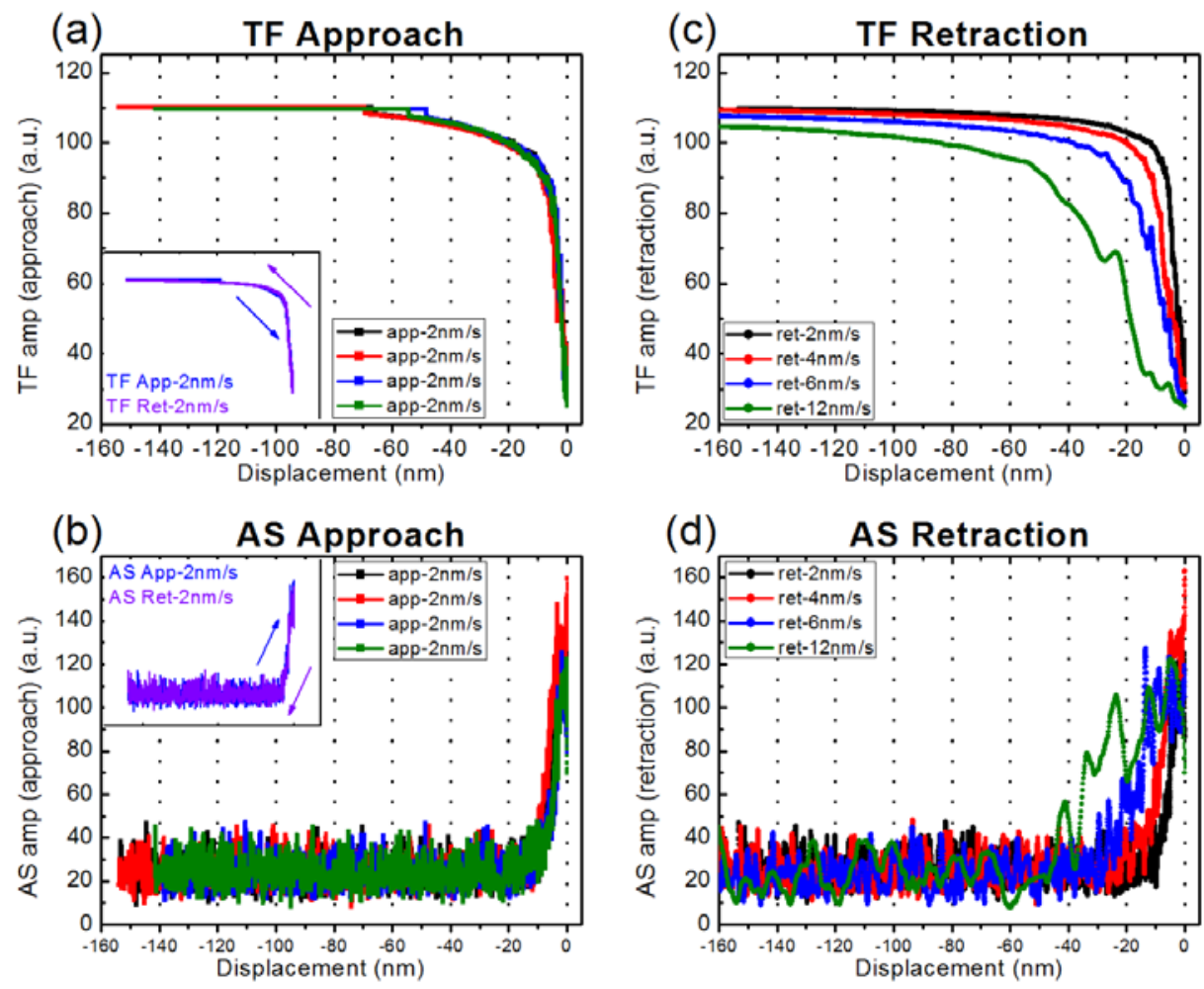

Figure 5.23: Approach ( $\mathrm{a}$ and b) and retraction (c and d) curves of TF and acoustic signals obtained on the gold surface using the same gold tip with a constant speed of approach ( $2 \mathrm{~nm} / \mathrm{s}$ ) but various speeds of retraction: $2 \mathrm{~nm} / \mathrm{s}$ (black), $4 \mathrm{~nm} / \mathrm{s}$ (red), $6 \mathrm{~nm} / \mathrm{s}$ (blue), and $12 \mathrm{~nm} / \mathrm{s}$ (green). Insertions: the approach/retraction curves of the TF (a) and acoustic (b) signals measured by moving the tip at the same speed of $2 \mathrm{~nm} / \mathrm{s}$.

Velocity chosen during the approach process was held constant at $2 \mathrm{~nm} / \mathrm{s}$. However, the tip was withdrawn from the surface at four different speeds: $2 \mathrm{~nm} / \mathrm{s}, 4 \mathrm{~nm} / \mathrm{s}, 6 \mathrm{~nm} / \mathrm{s}$, and $12 \mathrm{~nm} / \mathrm{s}$. The resulting curves of these four trials are shown in Figure 5.23 where the approach traces of the TF and acoustic signals are separately displayed on the left panel (Figures 5.23a and 5.23b represent TF (approach) and AS (approach), respectively; as a reference, insertions show the approach/retraction curves of the TF and acoustic signals 
obtained by moving the tip at the same speed of $2 \mathrm{~nm} / \mathrm{s}$ ), and the retraction traces are present on the right panel (Figures 5.23c and 5.23d represent TF (retraction) and AS (retraction), respectively). Tip motion is accurately controlled through the nanopositioner operated in close loop. With the use of the same velocity to approach, we have reason to believe that the tip has a similar experience in different approach attempts, and finally will stop at (or, will detach from) approximate the same height relative to the surface. The experimental data also verifies our assumption: approach traces of the TF and acoustic signals obtained in four different trials are overlapped (Figures 5.23a and 5.23b). In fact, we are more interested in the retraction curves because differences among traces acquired with varied speeds reveal the effect of velocity of tip motion. Indeed, we found measurable distinctions among the resulting retraction curves by changing the speed of tip motion: the tip which moves at a higher speed has to undergo longer traveling distance to escape from the sample (Figures 5.23c and 5.23d). The TF and acoustic signals vary in response to the interaction between the probe and the mesoscopic fluid layer. As the tip is withdrawn at high speed, the relaxation time of the fluid film to respond is probably not sufficient; as a result, liquid wrapped around the tip is dragged upwards with the lift of the tip. The faster the tip retracts, the more liquid moves along with the tip, and thereby the longer the TF retraction curve extends. Moreover, the dispersion of the correspondingly measured acoustic curves due to increasing the speed of tip motion is also clearly observed. Considering that the sound is generated by the vibrating tip in the fluid, elongation of the transition distance from the maximum to the minimum on acoustic curves provides evidence to support the idea that liquid moves 
along with the tip when retracting fast. In our experiments, the time constant of the lockin amplifiers were set to be $10 \mathrm{~ms}$, which was still suitable for the measurement with the highest velocity $(12 \mathrm{~nm} / \mathrm{s})$. However, a further increase in the moving speed may lead to loss of precision in detection of the TF and acoustic signals. Thus, in most cases, velocity of approach and retraction processes is kept at a low level $(1.5 \mathrm{~nm} / \mathrm{s} \sim 2 \mathrm{~nm} / \mathrm{s})$.

\subsubsection{Effect of Substrate Materials in the Acoustic Measurement}

At the end of this chapter, we expect to see the effect of substrate materials in acoustic measurements. Since the acoustic sensor is located under the substrate, sound waves created on the top of the sample surface will propagate through the bulk material to reach the sensor. It is possible that properties of the substrate material have effect in the detected acoustic signal. The sample used in this experiment was prepared by coating a thin layer of gold ( $\sim 40 \mathrm{~nm}$ of thickness) on one half of a glass cover slip. Prior to thermal coating of the gold film, the glass cover slip was cleaned using Piranha solution; and an ultrathin layer of chromium ( $3 \mathrm{~nm}$ of thickness) was deposited to the freshly cleaned glass surface for better adhesion of the gold. As shown in Figure 5.24a, the same gold tip was placed above the bare glass surface and the gold coated glass surface (an AFM image of such substrate is shown in Figure 5.24b), respectively. Approach/retraction curves were obtained by moving the tip close to and away from the surface at a constant speed of $2 \mathrm{~nm} / \mathrm{s}$ (ambient humidity was 30\%). The approach

process stopped once the amplitude of the TF signal dropped to $75 \%$ of its initial value, and the retraction process immediately started. 

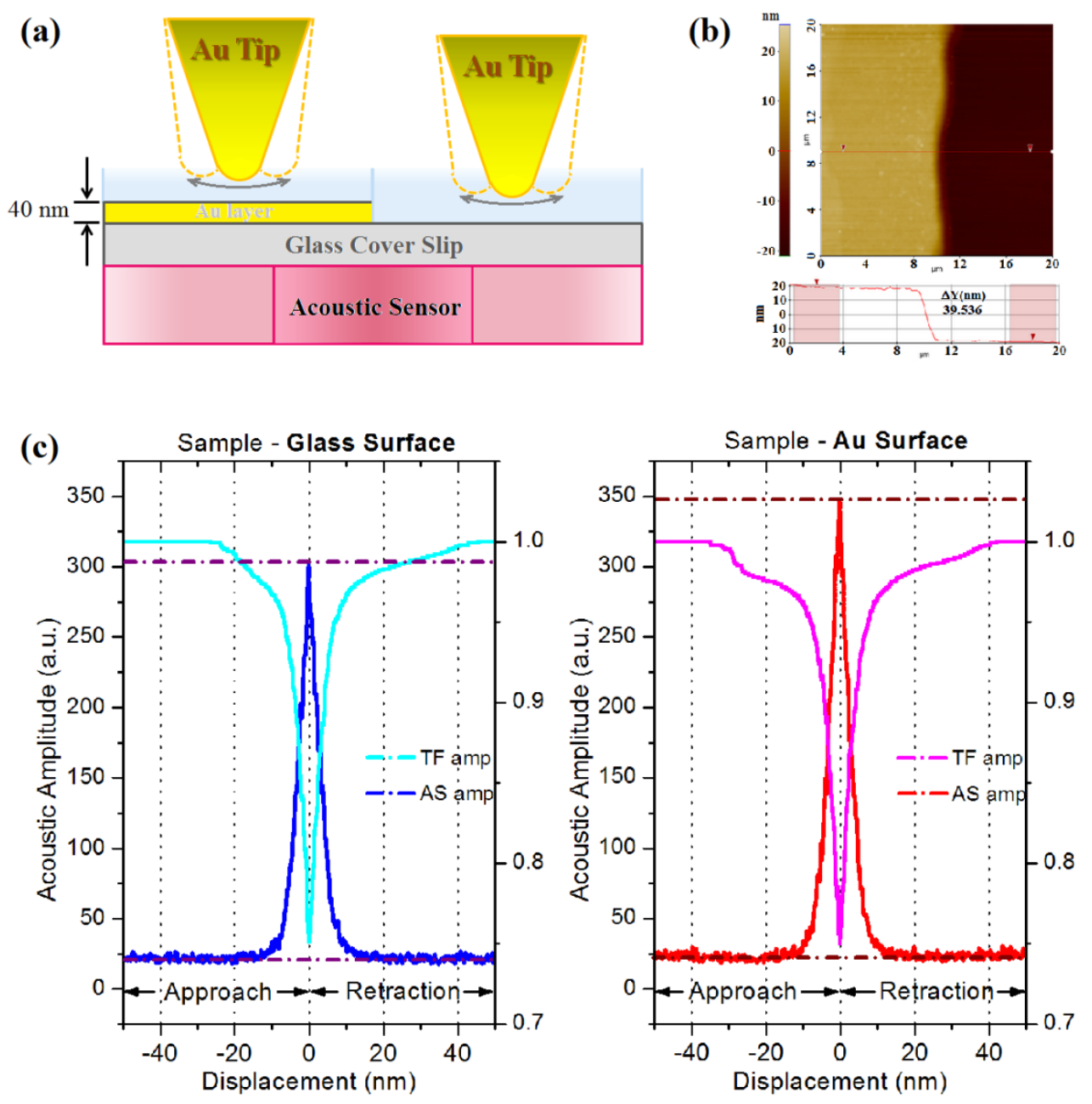

Figure 5.24: (a) Scheme of experimental procedure to obtain approach/retraction curves from the bare glass and gold-coated surfaces. (b) AFM image shows the thickness of Au coating is $\sim 40 \mathrm{~nm}$. (c) The TF and acoustic signals measured on the glass (left) and gold surface (right). The amount of the decrease in the TF signal approximates to the same value, whereas the maximum value of the acoustic signal on the gold surface is greater than that on the glass surface.

Here we reported an interesting result taken using our shear-force/acoustic microscope: the increase in the acoustic signal is more on the gold surface than that on the bare glass surface while the total amount of the decrease in the TF amplitude approximates to the same value ( $25 \%$ of the initial value). We displayed this 
experimental result in Figure 5.24c by taking an average of ten curves measured. The negative portion of $\mathrm{X}$-axis represents approach procedure; the positive portion represents retraction procedure. We plotted these two graphs using the same scale. It is evident that the baseline of the acoustic signal is roughly at the same level; whereas the maximum of the acoustic amplitude on the gold surface is greater than that on the glass surface. However, this contrast in the acoustic measurement was not present if the sample was replaced by the glass cover slip without any gold coating. This result implies that the amplitude of the generated acoustic signal is related to the material of the substrate. Despite that the underlying mechanism has not been well understood yet, the experiment can be considered as a worthy attempt to explore the possibility to use the acoustic technique for imaging substrates composed of different materials.

\subsection{Conclusions}

The distance-dependent interaction between the tip and the sample plays important role in SPM applications. We expect to extract information regarding the probe-sample interaction from the approach/retraction curves taken using the shear-force/acoustic nearfield microscope. Under ambient conditions, as the tip moves towards the sample, it first comes in contact with a mesoscopic fluid-like layer, which usually exists by attracting moisture from the atmosphere onto the solid surface. Immediately after the tip enters the fluid layer, a decrease in the amplitude of the tip's oscillation and a detected increase in the acoustic signal are typically observed. The increase in the acoustic amplitude at shorter probe-sample distance is attributed to the increasing phase lagging between the 
oscillating tip and the mesoscopic fluid layer. With further reduction of the gap between the probe and the surface, the tip comes into contact with the sample; as a result, the tip's oscillation is completely attenuated. Since the tip is stopped from vibrating, a decrease in acoustic signal is expected.

Several factors can affect the probe-sample interaction, and thereby leading to variations in the shape of the resulting approach/retraction curves. Herein we discussed impacts of tip geometry, relative humidity of the environment, the nature of sample surfaces, and velocity of the tip's movement on our shear-force/acoustic measurements. The tip with large radius of apex (chubby tip) is influenced more by the mesoscopic fluid layer than that having smaller radius of apex (sharp tip). An increase in environmental humidity leads to a growth of the contamination layer. Measured approach/retraction curves vary with the freshness and cleanliness of the sample and the tip. By performing cleaning of samples before their usage, hysteresis from the mesoscopic film is much reduced (even avoid). However, hysteresis may reoccur over time or after the tip in contact with the sample surface. Moreover, the effect of water affinity of sample surface is also discussed in our experiment. We proposed a hypothesis to explain the differences between the hydrophilic surface and the hydrophobic surface based on the measured approach/retraction curves. Extensions of the transition between the maximum and the minimum on the TF and acoustic retraction curve are found as the tip is moving at higher velocities. Additionally, monotonicity of the acoustic signal is helpful in interpreting the origin of the probe-sample interaction at different distances. Finally, we reported our observation of contrast in the acoustic measurement when placing the same tip above 
surfaces covered by different materials (i.e., a glass cover slip with a gold coating only on one half of the substrate). Despite that more experiments have to be carried out to explore the underlying mechanism, this is a worthy attempt for future use of the acoustic technique in imaging samples composed of different materials. 


\section{Chapter 6. Monitoring of Probe-Sample Mechanical Contact via Current Detection in Shear-Force/Acoustic Near-Field Microscopy}

\subsection{Introduction}

The study of the atomic-scale origins of friction, adhesion, lubrication and wear between interacting surfaces in relative motion is encompassed in the emerging field of nanotribology. In recent decades, studies of nanotribology at the fundamental level has benefited greatly from the invention of force microscopes, such as the atomic force microscope $(\mathrm{AFM})^{2}$, the friction force microscope (FFM) ${ }^{102}$ and the surface force apparatus (SFA) ${ }^{103}$. By measuring the force between a small tip and a sample surface, the use of an AFM was no longer limited by sample conductivity. The initial operation mode of an AFM obtains topographic images of a surface by maintaining a constant repulsive contact force between the end of the tip and the sample during scanning. Aside from basic operations for imaging purpose, a myriad of new operation modes have been developed. Among them, perhaps the most notable extension was the realization that lateral forces between the tip and sample could also be measured. This technique described as the FFM, along with other instruments (i.e., the SFA), shows promise to attain atomic-scale knowledge of frictional phenomena.

With the introduction of shear force microscopy (SFM) by Betzig et al. and Toledo-

Crow et al. ${ }^{68,104}$, detection of the so-called shear force between an oscillating tip and a solid surface provided great contributions to studies of nanotribology and other complex surface phenomena. The shear-force measurement technique had its origin during the development of the near-field scanning optical microscopy (NSOM), serving as an 
inevitable component to regulate the tip-sample distance. This technique relies on the following experimental observation: a tapered optical fiber is laterally vibrated at its resonance frequency. As the probe is brought into close proximity to a solid surface, its oscillation amplitude gradually decreases due to shear-force interactions. Typically, the tip-sample distance is required to be kept below $10 \mathrm{~nm}$. The implementation of shearforce distance control has been successfully achieved using different methods. Optical techniques, such as diffraction and interferometric means ${ }^{68,105}$, are commonly used to measure the oscillation amplitude of the probe. Alternatively, the use of a crystalline quartz tuning fork (with the probe attached to one of the tines) for monitoring the amplitude was demonstrated by Karrai ${ }^{35}$. This idea was based on the piezoelectric property of tuning forks which produce a piezoelectric voltage proportional to the amplitude of the vibration ${ }^{35,106-108}$. In practice the shear-force effect is sufficient for acquiring high quality images. However, there is still a lack of precise knowledge in relation to the nature of the shear-force interaction, which is strongly dependent on the actual tip-sample distance. A number of possible mechanisms have been proposed to explain the probe-sample interaction occurring in near-field microscopy, including van der Waals forces, electrostatic forces, viscosity, capillary forces, and actual probe-sample contact, depending on the experimental conditions ${ }^{68,69}$.

In our previous studies, we investigated features of the probe-sample interaction using the shear-force/acoustic microscope where an acoustic transducer was incorporated into the tuning fork-based shear force microscope setting ${ }^{109}$. It is able to independently and synchronously record electrical signals produced by the tuning fork (TF) and the acoustic 
sensor (AS). In addition to the shear-force effect on the probe, we could also monitor the dynamic response of the sample via the extra acoustic probing method. On the other hand, the acoustic technique could open up a new way to explain the shear-force interaction. For example, according to our acoustic data, we proposed that the phonon generation was one of dissipative channels of the shear-force interaction within a close probe-sample interaction region ${ }^{109}$. It is obvious that the probe's vertical position relative to the sample surface plays an important role in our experiments. Under ambient conditions, however, surface absorption leads to a contamination layer of a few nanometers thickness on the solid sample. Furthermore, when the fluid is highly confined between two hard surfaces (i.e., the probe and sample surfaces), it is likely to behave as solid ${ }^{101}$. This thin layer "perturbs" the probe-sample interaction, so that a precise location of the probe relative to the sample becomes difficult only based on measuring the shear-force and the acoustic signals. Herein, we present a simple strategy to detect a mechanical contact between a metal tip and a conductive sample by monitoring the contact current. Shear-force/acoustic curves were obtained together with the corresponding contact current. Detection of the contact current can also be helpful in interpreting the role of fluid-like layers in the generation of acoustic waves.

\subsection{Experimental Section}

The shear-force/acoustic microscope with the function of detecting contact current is schematically described in Figure 6.1 (the difference between Figures 6.1a and 6.1b is the way to apply a bias voltage for contact current detection). It mainly consists of three 
portions: (i) the "shear force" measuring system that comprises a tuning fork (TF) and a lock-in amplifier (Lock-in \#1; SR850, Stanford Research System); (ii) the "acoustic response” recording system, which consists of an acoustic sensor (AS) placed in intimate contact with the bottom surface of a sample and a lock-in amplifier (Lock-in \#2; SR850, Stanford Research System); (iii) the "contact current” monitoring system, including a low-noise current preamplifier (SR570, Stanford Research System) and a lock-in amplifier (Lock-in \#3; SR844, Stanford Research System). All the lock-in amplifiers share the same reference signal that is the excitation source of the TF. The time response of the lock-in amplifiers was set to be $10 \mathrm{~ms}$. Aside from using the lock-in amplifier, the output of the current preamplifier is also fed into an oscilloscope to monitor DC component of contact current (not shown in Figure 6.1).

The TF is of a commercially available type whose resonance frequency is $32768 \mathrm{~Hz}$. A gold tip was fabricated from a gold wire of $76 \mu \mathrm{m}$ in diameter (Au, purity 99.95\%, ESPI) by electrochemical etching in a mixture of $\mathrm{HCl}$ acid and ethanol (1:1 by volume). After etching, the tip was characterized with scanning electron microscope (SEM) and manually mounted to one of the tines of the TF. The conductive surface was prepared by coating a thin layer of gold ( $\sim 40 \mathrm{~nm}$ of thickness) onto a Piranha cleaned silicon wafer, following the deposition of chromium ( $\sim 3 \mathrm{~nm}$ of thickness) for better adhesion of the gold film. The gold coated silicon wafer was placed onto the AS (SE40-Q, Dunegan Engineering Consultants, Inc.). Dow Corning high vacuum grease was pasted between the sample and the sensor to improve the coupling efficiency of sound wave propagation 
In a typical operation, the TF was excited to laterally vibrate at its resonance frequency by applying an AC voltage across the TF's electrodes. Considering the fact that the probe-sample interaction depends on their separation, a good control of the tip’s movement must be implemented in experiments. The tip smoothly moves in the vertical direction (Z-axis) under coarse and fine control. Coarse control uses a set of fine-pitch screws (Newport; model: AJS100-2, travel: $50.8 \mathrm{~mm}$, sensitivity: $0.71 \mu \mathrm{m}$ ) which can rapidly reduce the gap between the tip and sample. Fine control was activated when the probe-sample distance is less than $50 \mu \mathrm{m}$. It is performed using a piezo-actuated nanopositioner (Nano-OP65, Mad City Labs, Inc.), which provides $65 \mu \mathrm{m}$ range of motion with nanometer precision. More importantly, the nanopositioner can be operated in close loop so as to eliminate the hysteresis arisen from the piezoelectric components. The whole microscope stage was sitting on a floating table to minimize mechnical perturbations. A piece of rubber cushion was placed between the stage and the table to further avoide undesigned disturbances. In a complete experimental cycle, the tip first approached towards the sample, and then retracted from the suface. Variations of electric signals generated by the TF and the AS simultaneously recorded as a function of the probe-sample distance were plotted as approach/retraction curves. The entire process of the tip’s approach and retraction was monitored through a CCD camera.

Sudden onset of current signal is considered as an indication of the mechanical contact between the gold tip and the gold surface. Here we used two configurations to connect the metal tip to a bias source. In one configuration (Figure 6.1a), a short gold tip is electrically connected to one of the TF electrodes ${ }^{110}$. Therefore, the bias voltage which 
is used to drive the TF is also applied to the tip. A negative bias applied to the sample is optional. In the other configuration (Figure 6.1b), a copper wire is directly soldered to a long gold tip to complete the electrical connection. An adjustable DC bias voltage ranging from $-5 \mathrm{~V}$ to $+5 \mathrm{~V}$ (usually, $50 \mathrm{mV} \sim 100 \mathrm{mV}$ ) is applied to the tip when grounding the sample. 


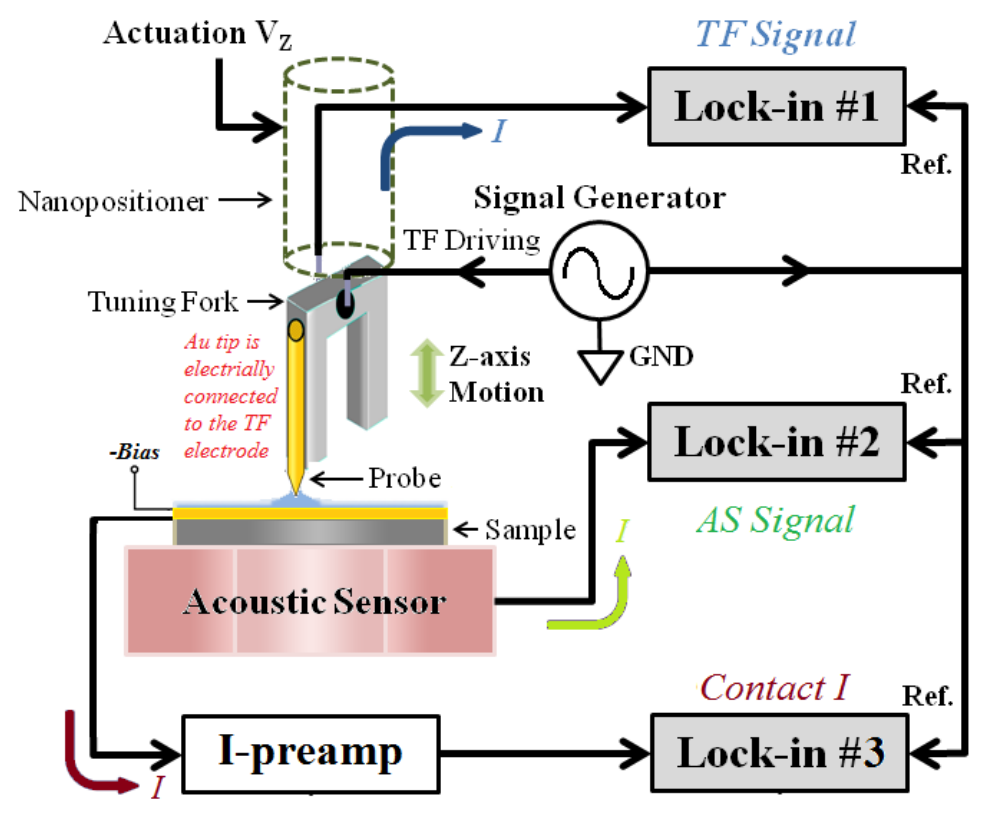

(a) Experimental Configuration 1

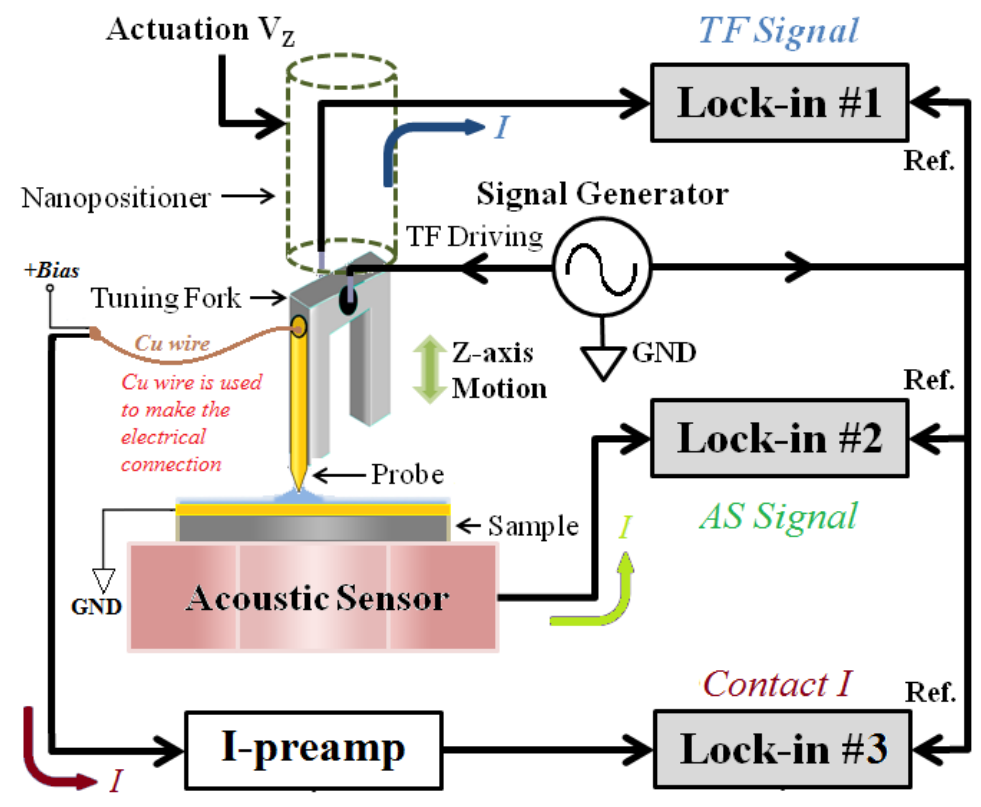

(b) Experimental Configuration 2

Figure 6.1: Schematic of experimental setups used for detecting contact current, along with the TF and the acoustic signals. In configuration (a), a short gold tip is electrically connected to the TF electrode and thereby the TF driving voltage is used to bias the tip. 
In configuration (b), a long gold tip is mounted on the TF and the bias is applied through an extra $\mathrm{Cu}$ wire soldered to the tip.

\subsection{Results and Discussion}

In one experimental cycle, the tip is first positioned at $\sim 15 \mu \mathrm{m}$ from the surface where it freely vibrates in air. The amplitude of the TF's oscillation is adjusted to be $\sim 10 \mathrm{~nm}$ by choosing an appropriate driving voltage. A fast approach $(15 \mathrm{~nm} / \mathrm{s}$ of velocity) of the tip is carried out to roughly find the so-called sample surface. Generally, once the amplitude of the TF's oscillation decreases by $\sim 1 \%$ of its initial value, the tip is immediately withdrawn from the surface. After the TF returns to its initial oscillation amplitude, the fast mode is replaced by the slow mode in which the tip moves towards and subsequently away from the sample with a constant velocity (i.e., $2 \mathrm{~nm} / \mathrm{s}$ ). Electric signals of the TF and the AS, together with the contact current, are synchronously measured with the change in the probe-sample distance. Since the current measurement is considered as a sign for the probe-sample contact rather than for the feed-back control, magnitude of the current had less importance. The configuration of the setup for current detection described in Figure 6.1a shows advantages over the one described in Figure 6.1b: for instance, tip attachment and electrical connection become easier; Q factor is not deteriorated by the extra wire connection; stability of the TF gets a noticeable improvement. Owing to these reasons, we adopted the former in most of our experiments demonstrated below. 


\section{A. The generation of acoustic waves and the occurrence of the probe-sample current}

Information is extracted from approach/retraction curves obtained while the gold tip approaches to and subsequently retracts from the gold sample surface at a constant velocity of $2 \mathrm{~nm} / \mathrm{s}$. The approach process continues until the amplitude of probe's oscillation reaches a specific value, which is referred to as the set point. Once the approach process stops, retraction process is carried out immediately until the tip is completely released from the surface. We carried out a series of experiments to gradually land the gold tip on the gold sample surface without damaging the tip apex. Figure 6.2 (ae) shows the approach/retraction curves obtained using the same tip and sample but choosing different set points. For example, the set point in Figure 6.2a is 90\%, which means the approach stops if the probe's amplitude falls to $90 \%$ of its initial value. The "zero" coordinates of horizontal axis in the graphs were arbitrarily selected, but all of plots followed the same rule: the origin of $\mathrm{X}$ axis was defined as the position where the amplitude of the TF signal drops to 95\% of the initial value during the approach process. In each individual graph, approach and retraction curves of the TF, the AS and current signals were plotted together, presented with different colors.

As the probe-sample distance decreases during the approach process, the tip enters the region in which the interaction between the tip and the sample occurs, leading to a decrease in the amplitude of the TF's electric signal on approach/retraction plots. Furthermore, we notice that all the graphs in Figure 6.2 tend to show the same trend that the amplitude of the acoustic signal increases as the tip's oscillation is attenuated due to shear-force effect. As shown in Figure 6.2a, the acoustic signal has started to rise above 
the noise level when the TF signal is only decreased by $10 \%$ of the initial value. From Figure 6.2b to Figure 6.2e, this tendency of the variation of the acoustic signal is getting clearer. In the meantime, we monitored the probe-sample current along with the TF and the AS signals. It is expected to observe current signal when the gold tip is in contact with the gold sample surface. Indeed, current peaks appear when the TF signal drops to about 26\% of its maximum value, illustrated in Figure 6.2e. To sum all graphs up in Figure 6.2, the current flow will not be detected unless the experimental set point is set to be or below 26\%. In addition, before the onset of current signal measured by the lock-in amplifier, there were no extra current components obtained using the oscilloscope. To protect the tip, we stopped lowering the set point once current signal was received. 
Approach/Retraction Curves (untilted Au tip)
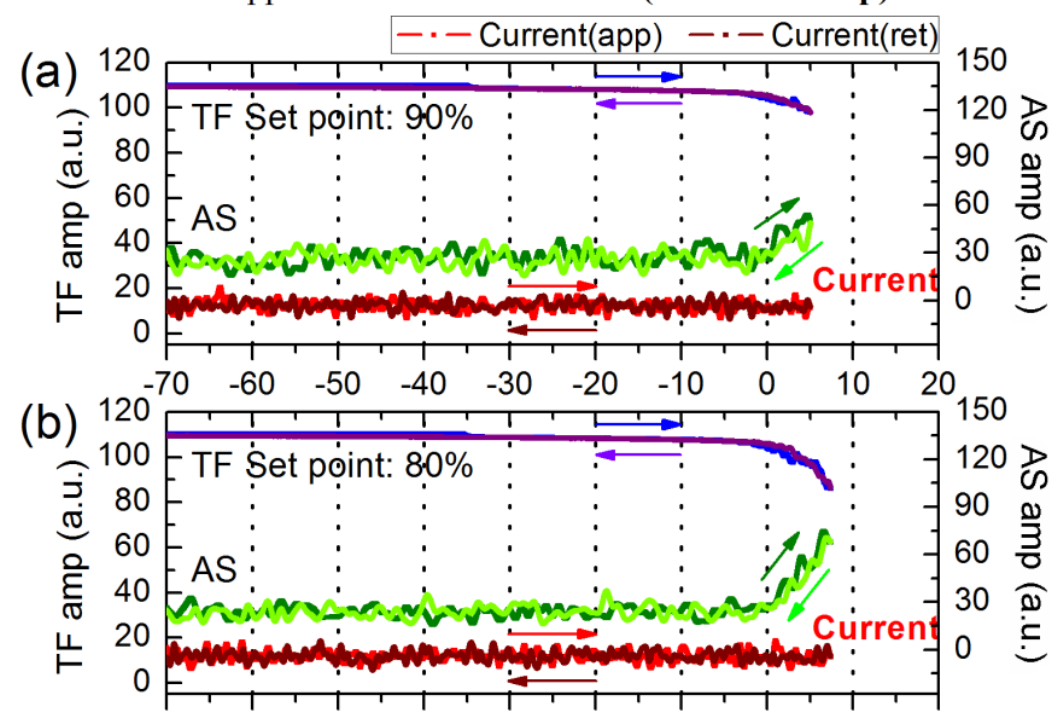

(c)
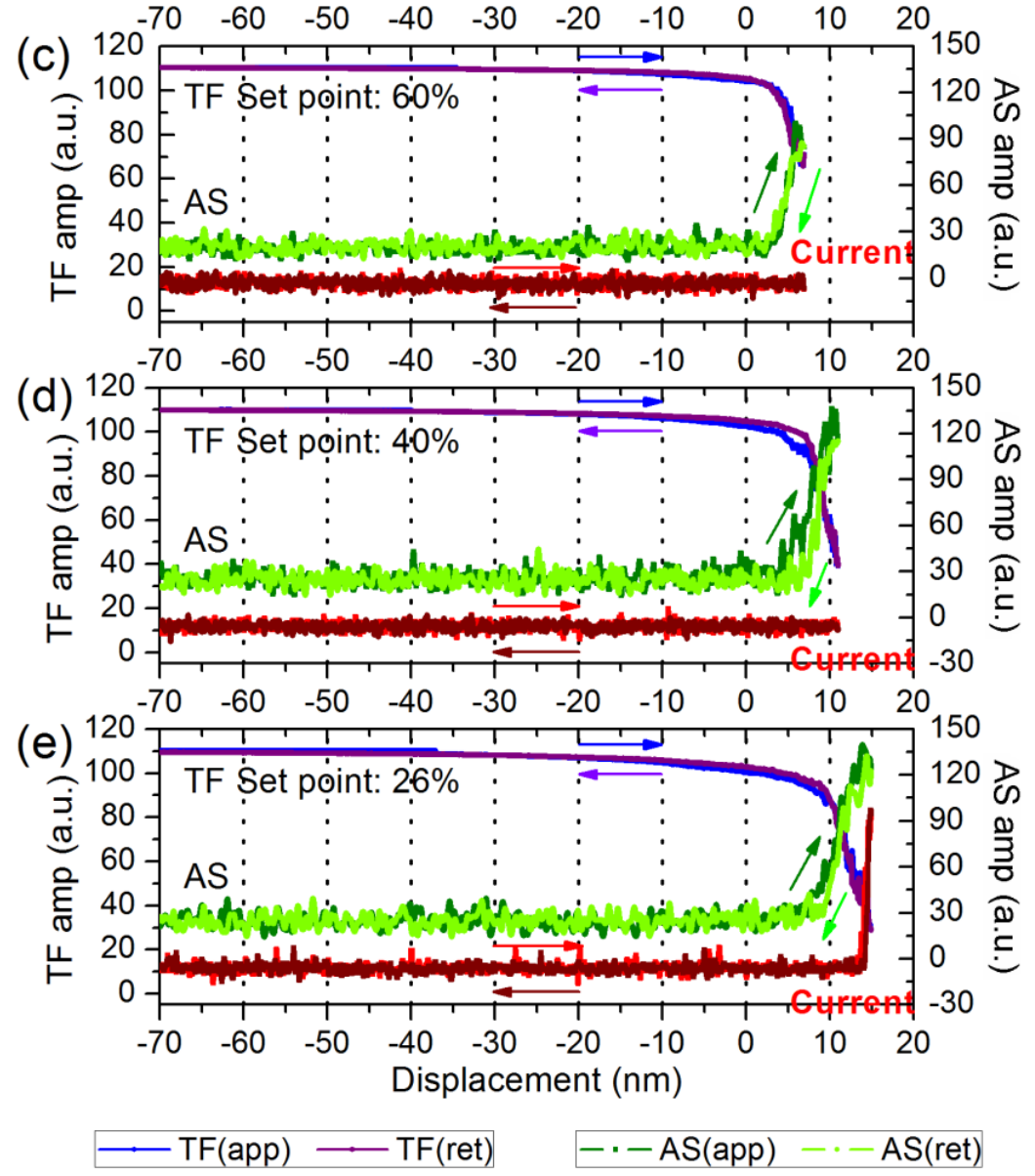

Figure 6.2: A series of experiments were carried out to gradually land a gold tip on a gold surface. The resulting approach/retraction curves of the TF signal, the acoustic response 
and the probe-sample current were obtained by setting different set points in turn: (a) $90 \%$; (b) $80 \%$; (c) $60 \%$; (d) $40 \%$; and (e) $26 \%$.

Under ambient conditions, absorption of water and carbohydrate molecules from air causes contamination of samples, leading to a fluid-like layer formed on hard surfaces. Our previous studies discussed the role of the liquid layer in the generation of acoustic waves, proposing that the immersion of the vibrating tip in the layer contributed to the generation of sound waves ${ }^{109}$. In close proximity to the surface, however, intermittent contact (tapping) of the probe with the sample may take place, producing acoustic signal. Therefore, it is difficult to definitely address the origin of sound in such region from approach/retraction curves of the TF and the AS signals. Direct observation of the probesample current is a valuable tool for indicating when the gold tip's apex encounters the conductive surface. Since the lateral vibration trajectory of the tip is slightly inclined relative to the surface, current pulses will be detected until the probe-sample distance is small enough for the tapping contact taking place. Our experimental data displayed in Figure 6.2e supported this viewpoint: the occurrence of current signal around the position of $+15 \mathrm{~nm}$ on horizontal axis can be considered as the sign of the mechanical contact between the tip and the surface. By combining with the rest of graphs in Figure 6.2, another important find is that the acoustic signal rises with a lack of the probe-sample current. It reveals that the generation of acoustic waves is not fully attributed to the direct contact of the probe with the surface. In fact, due to the existence of a mesoscopic fluidlike layer at ambient conditions, the vibrating tip suffers from attenuation in its amplitude 
when interacting with the fluid film; and acoustic waves are subsequently generated as a result of the probe dissipating its energy in the liquid.

The timing of the contact current to be detected probably vary with the state of the tip, such as, the amplitude of the tip's oscillation and the tilt of the tip's axis relative to the sample ${ }^{69,111}$. As proposed by Gregor et al., a slight tile of the probe's axis relative to the surface normal leads to intermittent knocking of the tip on the surface ${ }^{69}$. Differing from large amplitude of oscillation ( 200 nm) used in Gregor's study, in most of our cases, the amplitude of the vibrating probe in air was kept small $(\sim 10 \mathrm{~nm})$; the tip was also carefully adjusted to be perpendicular to the surface through a tripod under monitoring by a CCD camera. However, it is difficult to assure the tip completely parallel to the normal to the surface. It should be noted that a small tilting angle between tip end and solid sample surface may give rise to an early onset of the probe-sample current. As shown in Figure 6.3, if the tip is carefully aligned in the vertical direction, a probe-sample current appears unless the measured TF signal falls to $26 \%$ of its initial value (Figure 6.3a); whereas after intentionally tilting the tip, current pulses are observed when the tip's amplitude drops to $43 \%$ of its maximum value (Figure $6.3 \mathrm{~b}$ ). In comparison with the experiment done with the aligned tip, current signal appears earlier when using the tilted tip. This observation suggests that the occurrence of the probe-sample current is sensitive to the tilt of the tip, which may cause short effective distance $\left(d_{e}\right)$ between the very end of the vibrating tip and the hard surface (Figure 6.3). A rude adjustment of the tip's axis relative to the sample surface will result in unexpected contact between the metal tip and the conductive sample, which takes place in an early stage of the approach process. 
Additionally, the appearance of current signal also depends on surface roughness and cleanliness. In our experiment, the topographic image taken using atomic force microscope (AFM) indicated the gold surface was smooth.
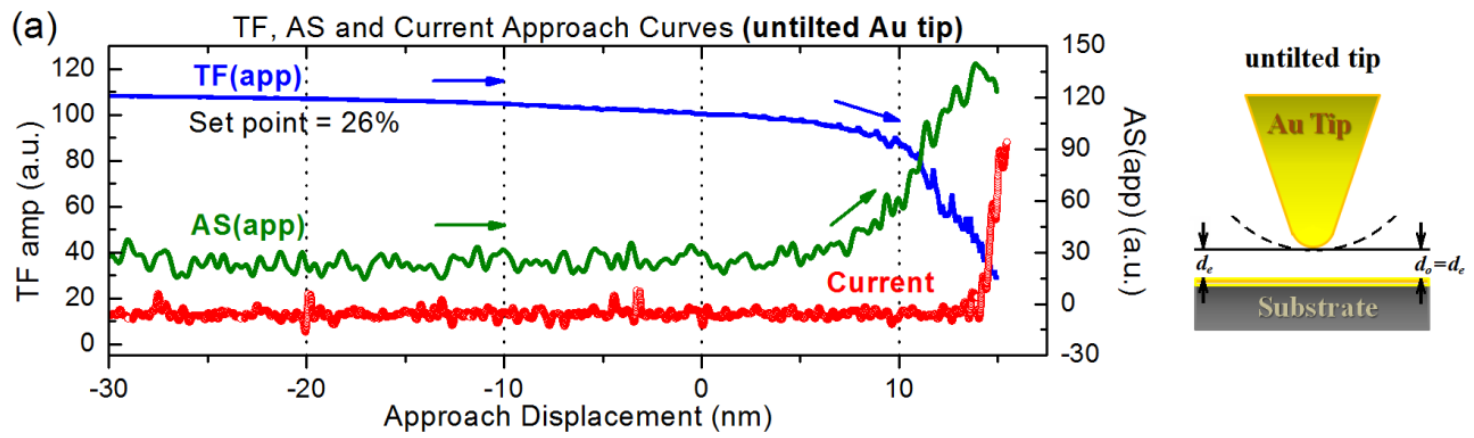

(b)

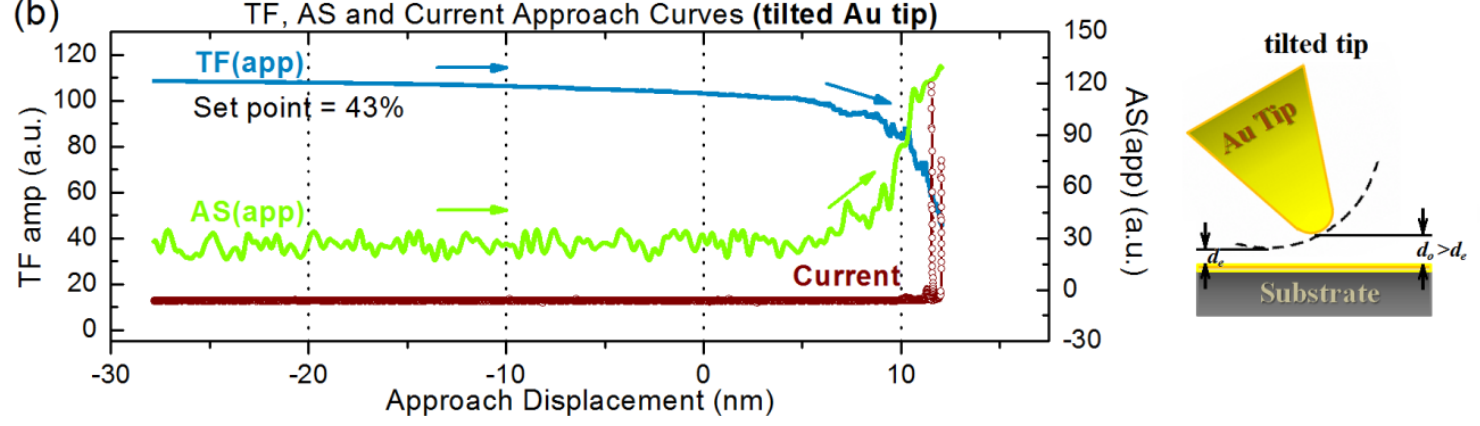

Figure 6.3: Approach curves of the TF, the AS, and the probe-sample current signals obtained using the same gold tip: (a) if the tip was carefully adjusted, current signal was observed when the set point of the approach process was set to be $26 \%$; (b) if the tip was tilted on purpose, current signal occurred earlier when the set point was $43 \%$. The term $d_{o}$ represents the distance between the very end of the tip and the hard surface. As the tip is driven to oscillate, the term $d_{e}$ represents the effective (shortest) distance between the vibrating tip and the surface. For a given $d_{o}$, if tip is perpendicular to the surface $d_{e} \approx d_{o}$; if the tip is tilted $d_{e}<d_{o}$.

\section{B. Characterization of acoustic curves with help of the probe-sample current}

One potential benefit of adding an acoustic sensor to the conventional shear-force microscopy is to provide extra information regarding the probe-sample interaction. The acoustic measurement reflects the response of the sample (including the absorbed fluid 
layer on the solid surface) when the probe comes into contact with it. In general, the detected acoustic signal strongly depends on the probe-sample distance. As the distance is changed in experiments, the type of interactions between the probe and the sample can vary from one to another, resulting in corresponding changes displayed on the TF and the acoustic curves. Therefore, an estimation of the actual position of the tip above the sample is a prerequisite to gain a better understanding of the interaction and its origin at different probe-sample distances. For metal tips and conductive surfaces, it is possible to detect a mechanical contact between them by measuring an electric current flow (the current direction depends on the applied bias). Herein current detection is integrated into our shear-force/acoustic microscope to further explore and verify the advantages of using the acoustic probing technique to interpret the probe-sample interaction.

During approach/retraction processes, the probe-sample interaction can be greatly influenced by several microscopic factors, such as tip’s properties and local situation of sample surfaces. A combination of these parameters will lead to variations of obtained approach/retraction curves in morphology. Our analysis is based on the approach curves of the TF, the AS and current signals, aiming to find out similarities among different curves rather than to focus on each individual curve. Figure 6.4a shows a typical approach curve which is often observed in our studies. Here the origin of the horizontal axis is artificially defined as the position where the approach process terminates (it may not be the actual location of the hard surface). The TF signal exhibits a monotonic dependence on the probe-sample distance: the closer the tip to the sample, the smaller the vibration amplitude. However, the variation of the AS signal with the change in distance 
is not as simple as that displayed on the TF curve. The most significant characteristic of the AS signal is the "big bump" presented near the end of the approach curve. Current signal is only observed at the last stage of the approach operation.

We re-drew the same approach traces of the TF, the AS and current signals in Figure 6.4c by resetting the "zero" coordinates to be the position where the amplitude of the TF signal drops to $95 \%$ of its maximum value so as to avoid a bias in judgment of the real solid surface. Upon the monotonicity of the acoustic curve (related to the appearance of the "bump"), we can divide the entire transition process of the measure signals into three portions labeled on the graph. As an example, portion (I) of the approach process is located in the region from $-40 \mathrm{~nm}$ to $+20 \mathrm{~nm}$, where the TF signal slightly decreases with no obvious change in neither the AS signal nor the current. Portion (II) is marked in the region from $+20 \mathrm{~nm}+35 \mathrm{~nm}$, where the TF signal shows a monotonic decrease in the amplitude with a steeper slope. It is evident that an increase above the noise level in the acoustic measurement coincides with the attenuation of the tip's oscillation, which is referred to a negative correlation. However, the current is still not detected yet. Portion (III) falls in the region from $+35 \mathrm{~nm}$ to $+40 \mathrm{~nm}$, where the most significant difference from other two is observation of the probe-sample current in the last $2 \mathrm{~nm}$. The correlation between the TF and the AS signals also switches to positive, which means the acoustic signal varies in the same trend as that of the change in the TF signal. 
(a) Approach Process Set point $=\mathbf{2 4} \%$

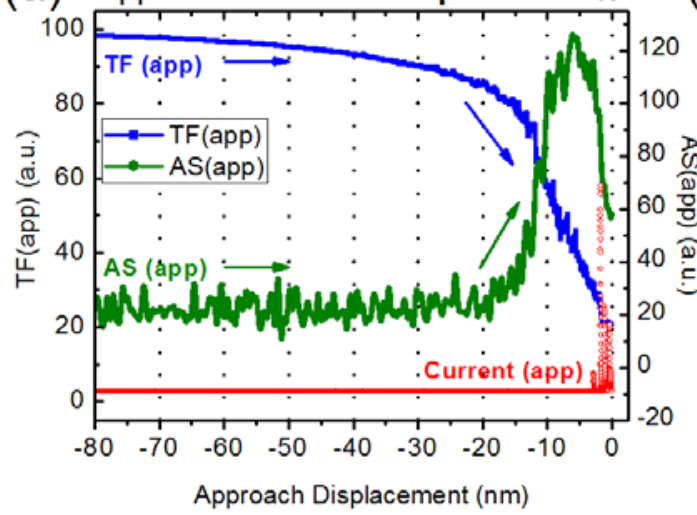

(b) Retraction Process Set point $\mathbf{= 2 4 \%}$

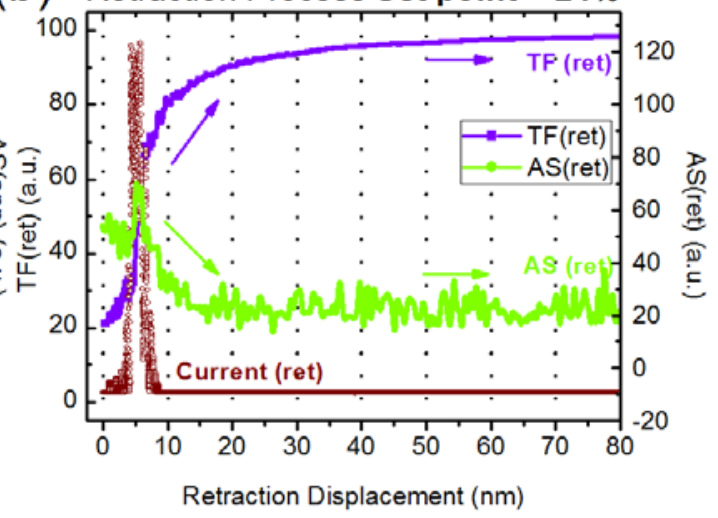

(c)
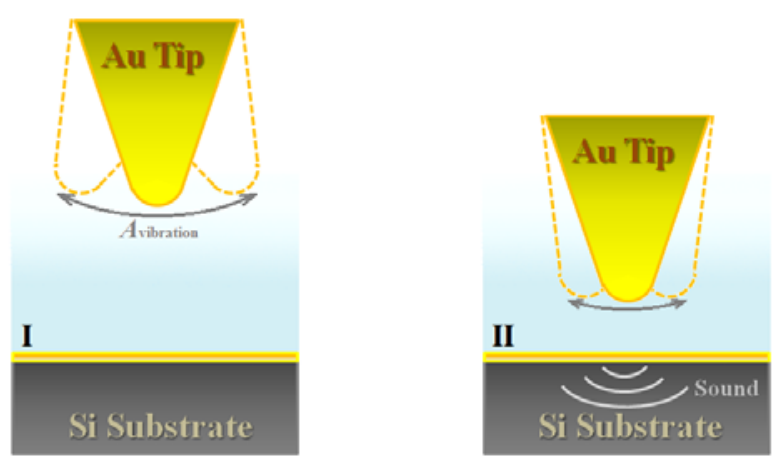

Approach $]$
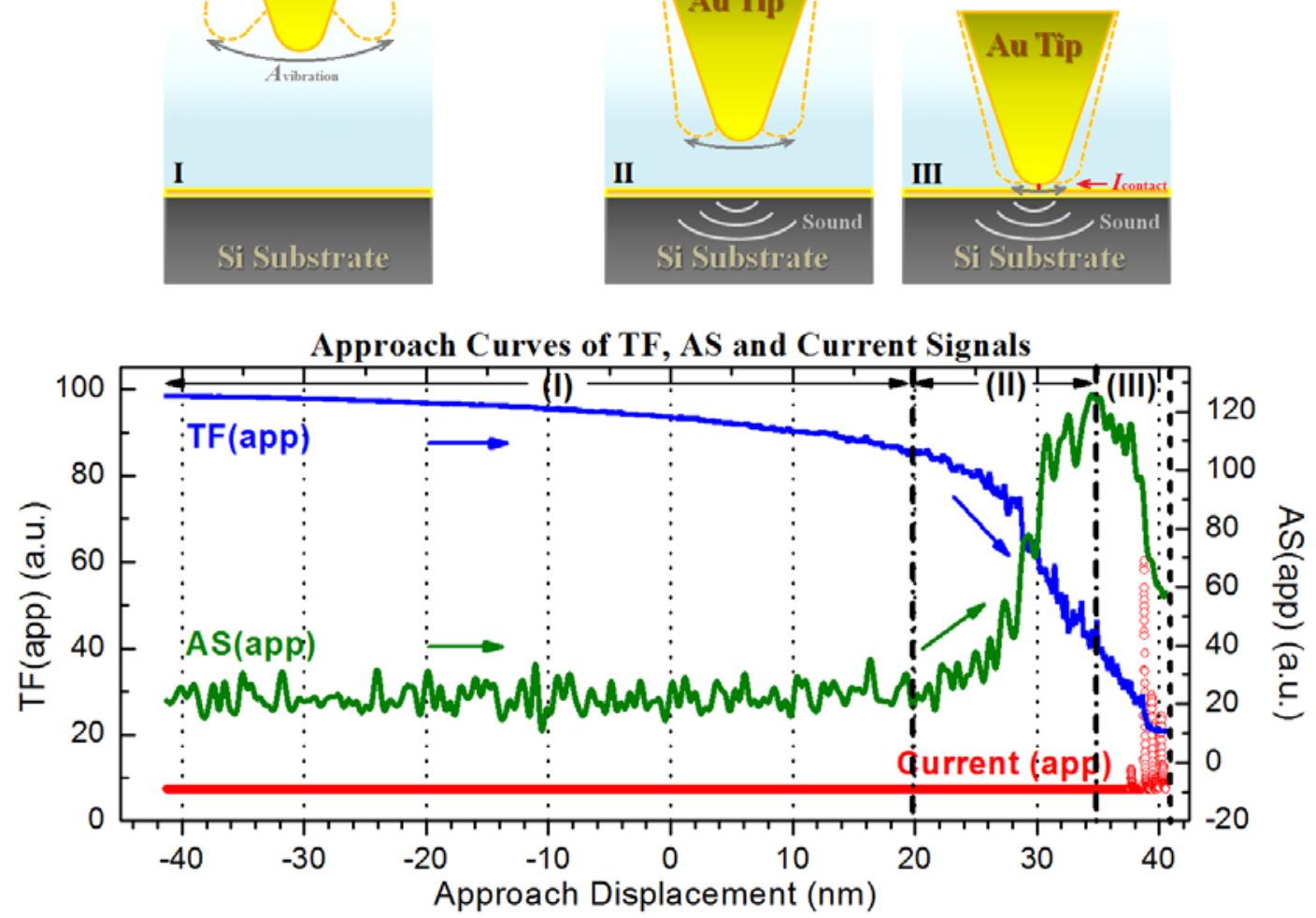

Figure 6.4: A typical approach/retraction curve obtained by concurrently measuring the TF signal, the acoustic response and the probe-sample current (the set point was 24\%): (a) approach and (b) retraction curves plotted by choosing the origin of $\mathrm{X}$ axis as the position where the approach process completes or the retraction process started. (c) The same result is re-drawn by selecting a new origin of $X$ axis (the position where the TF amplitude drops to $95 \%$ of its maximum) in order to avoid a misunderstanding in locating 
the actual hard surface. The upper scheme shows a possible explanation for the probesample interaction in different regions.

These curves acquired using various probing techniques (shear-force measurement, acoustic sensor, and probe-sample current detection) provide a qualitative impression on the behavior of the tip interacting with the sample when approaching toward and subsequently retracting from the surface. The tip initially oscillated at its resonance frequency in air, and the amplitude of the measured TF signal reached the maximum. The tip's vibration in atmosphere also created a sound signal detected by the acoustic sensor as a non-zero background. After the approach process being started, the tip was brought closer to the sample. It experienced a slight damping of its oscillation amplitude, which perhaps originates from the impact of long-distance forces (i.e., electrostatic forces). However, no significant increase above the noise level was observed on the acoustic curve. One possibility is that the generated sound is weak and hidden in the background noise. Another plausible explanation is that, considering that the gap between the tip and the sample is still large, sound waves cannot be efficiently created with a lack of actual probe-sample interactions (in portion I). With the approach process being continued, the tip penetrated into an absorbed fluid layer existing on the solid surface when exposing to the ambient air. Accordingly, the tip’s oscillation was further attenuated in the liquid due to forces such as viscous drag and capillary force; whereas acoustic waves, produced as a form of dissipation happened to the tip, were propagating through the substrate and were received by the sensor. In the mesoscopic fluid film, sound generation becomes more efficient due to a greater phase lagging between the vibrating probe and the mesoscopic 
fluid at shorter probe-sample distance. Therefore, the acoustic signal exhibited an upward tendency while the tip approaches to the surface (in portion II). The missing of current signal provides evidence that sound can be created even without tapping of the tip onto the hard surface, which is consistent with our previous studies ${ }^{109}$. Finally the approach process ended up with a mechanical contact of the tip with the solid surface, which was indicated by the observation of the probe-sample current. The spikes of current suggest that the tip is knocking on the sample. The solid-solid contact disturbed the lateral oscillation of the probe and led to friction, as evidenced by a corresponding decrease in both the TF and the AS signals (in portion III).

In the subsequent retraction process (Figure 6.4b, the origin of the horizontal axis represents the position where the tip starts to detach from the surface), the tip was gradually released from the surface. The birth of the bump on the acoustic curve could be attributed to a transition of the tip from a relatively static state with respect to the sample to a tapping mode. The occurrence of the maximum current supports this explanation. Moreover, we notice that there is a steep increase in the TF signal accompanied with peaks of the acoustic measurement and current signal. One possible interpretation is to consider the situation that the tip is tapping the hard surface; meanwhile, the surface is also pushing against the tip. Thus a sudden increase in both the TF amplitude and the acoustic signal takes place at that location as a consequence of this interplay between the tip and the sample. Compared with approach curves, which mainly reflect the probesample interaction in a mesoscopic fluid layer, the shape of retraction curves is closely related to the local properties of the sample surface. Since the retraction curve is probably 
obtained after the probe-sample contact occurs, aside from the influence of the absorbed liquid film, surface adhesive and frictional properties at the position where the tip contacts the hard surface will affect the resulting curve. Furthermore, there is a chance to pick up particulate materials from the surface due to the mechanical contact of the tip with the sample. Unpredictable perturbations on the surface, induced by the tip’s touch, seem to have impacts on the acoustic signal detected during retraction. By comparison, the approach curves are likely more reliable to be adopted for characterizing the mesoscopic fluid film and studying the probe-sample interaction occurring in it.

The results described above are repeatable. As shown in Figure 6.5a, by changing to another gold tip, approach curves similar to the typical one were observed using the same experimental setup described in Figure 6.1a. Alternatively, the experimental configuration mentioned in Figure 6.1b is also feasible for detection of the probe-sample current; and the resulting approach curves displayed in Figure 6.5b show consistency with those in Figure 6.5a. By comparing these two graphs, the transition distance of the TF signal in Figure 6.5b is shorter. It may arise from the drawback of its own experimental setup: attachment of a long tip and electrical connection through an extra wire could dramatically lower stability and sensitivity, and thus making the measurement of a slight variation in the TF amplitude difficult. Additionally, the presence of permanent contact current at the end in Figure 6.5b may imply the landing of the tip on the solid surface. Under SEM we found the tip used in Figure 6.5b has a larger diameter than the one used in Figure 6.5a. An obvious consequence of using the tip with large diameter is that: saturation of current signal is easier if the tip is chubby. However, the 
saturated current may not represent complete rest of the tip’s oscillation. There perhaps exists a slight sliding of the tip over the surface at the location where they are in contact with each other, and therefore producing the high-level acoustic output.
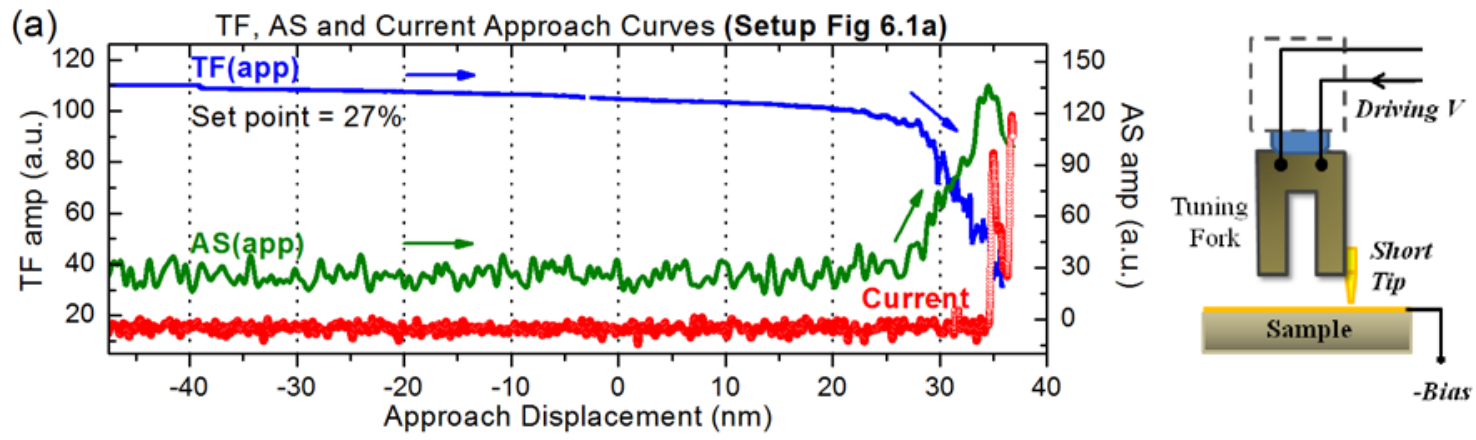

(b)

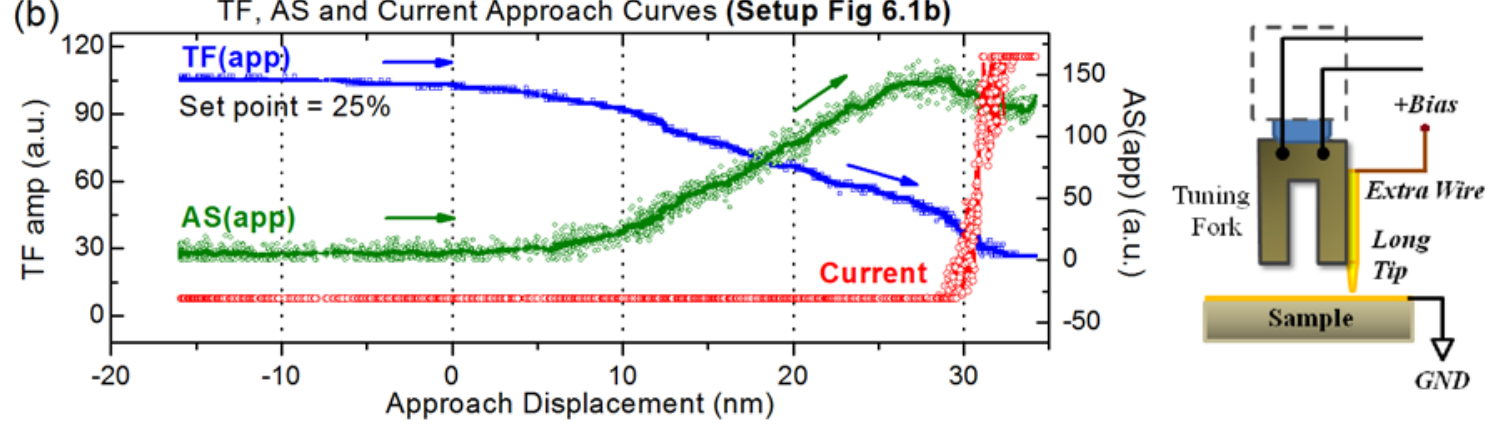

Figure 6.5: (a) Approach curve of the TF, acoustic and current signals obtained using the experimental setup mentioned in Figure 6.1a. (b) Approach curve of the same signals measured using the experimental configuration described in Figure 6.1b.

\subsection{Conclusions}

We demonstrated that detection of the probe-sample current can be successfully implemented into the shear-force/acoustic microscope. Therefore, multiple probing techniques were concurrently utilized to investigate the tip’s behavior and the probesample interaction for near-field applications. Information was extracted from the approach/retraction curve, which was the measurement of electric signals plotted as a 
function of the probe-sample distance. According to our experimental data, one of the most compelling results was that acoustic waves were generated before a mechanical contact of the gold tip with the gold surface. In fact, during the entire approach process, the probe exerts external forces, which cause the net decrease in the amplitude of tip's oscillation. The combination of these forces is referred to the so-called shear force. However, the origin of shear force can be diverse, depending on the actual probe-sample distance. Acoustic technique as a promising tool was added to help explore the nature of the distance-dependent shear-forces effect. Furthermore, in order to approximately locate the actual site of the hard surface relative to the tip' vertical position, one solution is to detect the probe-sample current. By applying a bias across the conductive tip and sample, the occurrence of current signal indicates a mechanical contact between them. By combining our observation of the TF, acoustic and current signals, we found that the mesoscopic fluid layer on solid surfaces played an important role in the probe-sample interaction. It caused a decrease in probe's oscillation amplitude through forces like viscous drag and capillary forces. On the other hand, owing to the existence of such layer, the vibrating tip in the film created sound as a form of energy dissipation of the tip's mechanical motion. Current monitoring suggested that there is no need for solid-solid contact in the generation of sound waves. Detection of the probe-sample current was also useful in interpreting the behavior of tips in close proximity to the surface. With the help of current signal, we found the negative correlation between the TF and the acoustic signal is due to the presence of the absorbed fluid layer; and the positive correlation is attributed to the direct contact of the tip with the hard surface. The onset of contact 
current was also affected by the adjustment of the probe's axis. By keeping the tip’s vibration amplitude small ( $~ 10 \mathrm{~nm})$, a purposive tilt of the tip relative to the normal to the surface caused an early appearance of current signal. Detection of the probe-sample current provides a qualitative impression on the tip’s behavior when approaching towards the substrate surface. Therefore, we can gain a clearer insight into how the probe interacts with the surface (including the absorbed fluid film) at different probe-sample distance, which plays an important role in near-field microscopic imaging. 


\section{Chapter 7. Acoustic Detection with Probes Immersed in Liquid Droplets}

\subsection{Introduction}

Quartz tuning forks (TFs) have been successfully implemented into scanning probe microscopy (SPM) to measure surface topography with atomic resolution. The TF is used as a shear force detector, while the SPM tip acts as a shear force pick-up. The idea behind the control of the tip's position related to the sample surface is based on the following experimental observation: within the shear-force interaction range the tip’s oscillation amplitude is damped out during approaching to a sample surface. The origin of shear forces is not fully understood. A number of possibilities have been put forward, including van der Waals forces, electrostatic forces, viscous drag in a contamination layer, and short-range mechanical contact (knocking).

In conventional shear-force detection, the TF is the only sensor whose response characteristics (i.e., the oscillation amplitude and the resonance frequency) reflect the effects of tip-sample interactions on the tip. Our shear-force/acoustic near-field microscope provides an alternative method to study probe-sample interactions by taking advantage of acoustic waves resulted from the lateral vibration of the tip in proximity to the sample surface. It differs from other acoustic techniques in which the probe is driven perpendicular to the sample or is kept stationary while the acoustic wave is applied to the

sample $e^{37,112}$. With the placement of an acoustic sensor under the sample, we can directly "hear the sound of shear forces" as the tip oscillates parallel to the surface. In operation, both signals from the TF and the acoustic sensor are simultaneously and independently 
captured as a function of the tip-sample separation. They can offer richer information about these two interacting bodies (the probe and the substrate including the contamination layer) than either one by itself ${ }^{73}$. For example, monotonicity of the acoustic signal is helpful to interpret the behavior of the tip in the last step of approach process.

The existence of a contamination layer composed typically of moisture and other hydrocarbon molecules on the sample surface exposed to air has been widely accepted $^{70,72}$. A proper characterization of the behavior of this liquid layer at surfaces is essential to study adhesion, surface wetting, and sliding friction. A complete understanding of the dynamics of fluid-like layers at liquid-solid interfaces is also desirable for SPM applications where probe-sample interactions mediated by the absorbed layer are exploited for feedback control. We previously reported the use of acoustic probing technique for studies about the role played by the contamination layer in the probe-sample interactions ${ }^{73}$. Experimental results suggested that the forced lateral motion of the tip submerged in the liquid film can be responsible for the generation of acoustic waves. An abrupt change in the TF signal during approach indicated that capillary force (although this may not be the main contribution to the shear force) builds up as the tip enters the fluid-like layer. In the presence of water films condensed onto sample of tip, a sudden formation of a liquid meniscus occurs to fills the small tip-sample gap. Additionally, when a fluid is confined in a nanometer-sized gap, an increase in its effective viscosity and the manifestation of an elastic behavior are produced. These two effects are more characteristic of a complex fluid than of an ideal Newtonian fluid ${ }^{113,114}$. 
Therefore, we considered a distance-dependent viscoelasticity to explain the generation of sound: closer to the sample, the acoustic signal consistently strengthens due to an increase in the viscoelasticity of the confined liquid.

Despite the fact that dynamics of a liquid under high confinement could differ from bulk properties, our measurements performed in bulk liquid could provide knowledge of the mechanism of sound generation analogous to that in the mesoscopic regime. The aim

of this chapter is to experimentally investigate the origin of acoustic waves coupled to the acoustic sensor located under the sample. Experiments are carried out with the tip immersed in a small volume of liquid. Frequency spectra of a TF where a cylindrical optical fiber is attached as a probe are measured using the shear-force/acoustic technique. Diameters of optical fibers and viscosities of liquids are varied on purpose to explore their influences on the creation of acoustic waves.

\subsection{Experimental Section}

Quartz TFs are commercially available products, packaged in vacuum with standard frequency of $32768 \mathrm{~Hz}$. In application, the TF was uncovered from its vacuum lid. A flat cleaved optical fiber was carefully mounted to one of the TF prongs. The fiber was protruding $\sim 1 \mathrm{~mm}$ outside the prong. Diameters of glass fibers were reduced through chemical etching with a buffered HF (BHF) solution ${ }^{67}$. The fiber is thinned rather than sharpened using the BHF solution with the volume ratio of $\mathrm{NH}_{4} \mathrm{~F}: \mathrm{HF}: \mathrm{H}_{2} \mathrm{O}=2: 1: 1$. Resulting diameters of etched fibers were measured from their optical images. The glycerol-water solutions with glycerol concentration varying from $0 \%$ w.t. to $50 \%$ w.t. 
were prepared by mixing calculated weights of glycerol and of distilled water. The viscosity values of glycerol aqueous solutions were given in literature ${ }^{115}$.

Our goal is to monitor the probe’s oscillation amplitude (assumed here to be proportional to the TF signal) as well as the corresponding acoustic response while the cylindrical fiber is oscillating in a liquid, for which we use the shear-force/acoustic experimental setup described in Figure 7.1. The protruding fiber was dipped into a droplet $(\sim 5 \mu \mathrm{L})$ of glycerol aqueous solution placed on a mica disk. We chose the mica disk as the substrate because the liquid can easily spread to the maximum on mica so that the effect of droplet geometry could be minimized. The penetration depth was varied using a set of fine-pitch screws (Newport; model: AJS100-2, travel: $50.8 \mathrm{~mm}$, sensitivity: $0.71 \mu \mathrm{m})$ associated with a nanopositioning stage (Nano-OP65, Mad City Labs, Inc.).

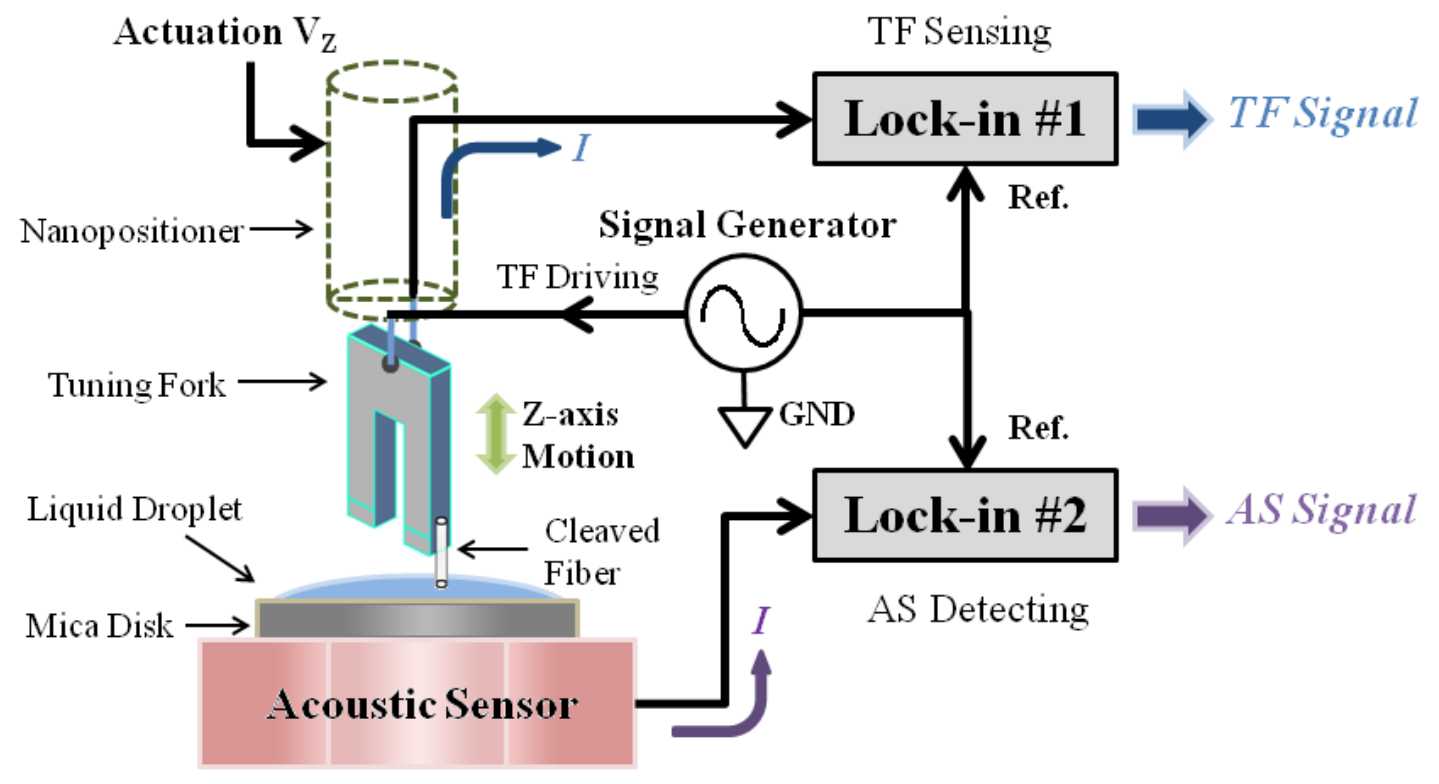

Figure 7.1: Schematic of experimental setup used to detect the TF and acoustic signals when the cleaved fiber is laterally oscillating in a droplet of glycerol aqueous solution ( $~ 5$ $\mu \mathrm{L}$ ) placed on a mica disk. 
The TF was electrically excited through its electrodes. When AC driving voltage with frequency $f_{d}$ is applied across the TF electrodes, the fork prongs are oscillating with the same frequency $f_{d}$. Because of the piezoelectric effect, an induced current in the TF proportional to the oscillation amplitude of the prong can be output and detected. By sweeping the driving frequency ( $f$ ) and recording the corresponding outputs $(I)$ from the TF and from the acoustic sensor via lock-in amplifiers (SR850, Stanford Research System; selecting a time constant of $10 \mathrm{~ms}$ ), we obtain the information about the TF's oscillation amplitude and the acoustic response with the change in frequency, respectively. The first is regarded as the vibration spectrum ( $f$-I curve). Under the resonant condition, the TF prong attains the largest displacement that corresponds to the maximum current amplitude in $f-I$ curve. The sweeping frequency range depends on the individual TF, because the loading of a probe introduces variations. The excitation voltage is adjustable. All experiments reported herein were preformed under temperature $\sim 23{ }^{\circ} \mathrm{C}$ and humidity $\sim 45 \%$.

\subsection{Results and Discussions}

A $125 \mu \mathrm{m}$ diameter cylindrical fiber was mounted to a TF excited to oscillate parallel to the mica surface; this was achieved by electrically driving the TF with an AC voltage of $40 \mathrm{mV}_{\mathrm{rms}}$. The protruding portion of the fiber was gradually immersed into a drop of prepared solution with a depth ranging from $0 \mu \mathrm{m}$ to $280 \mu \mathrm{m}$. Figure 7.2 shows typical vibration spectra of both TF and acoustic signals in response to frequency sweeping at 
various penetration depths (40 $\mu \mathrm{m}, 120 \mu \mathrm{m}, 200 \mu \mathrm{m}$ and $280 \mu \mathrm{m})$ in distilled water (glycerol concentration is $0 \%$ w.t.). It took $20 \mathrm{~s}$ to record one trace.

In principle, the TF can be either mechanically excited by an additional piezoelectric element (MD) or electrically driven through the fork electrode pair (ED). The ED mode has advantage over MD mode in that external components are not required to actuate the TF or to sense its motion. However, an antiresonance characterized by a minimum in the current at a frequency above the resonance frequency displays on the $f-I$ curve as a result of the intrinsic capacitor of the $\mathrm{TF}^{116}$ (Figure 7.2a). The directly measured current cannot truly reflect TF mechanical motion. To overcome this problem, as mentioned in Chapter 3, a simple method has been proposed to convert the measured electrical signal of the TF into the signal induced by its mechanical motion ${ }^{64}$. This method allows modeling the mechanical motion of the TF as a forced harmonic oscillator, finding its equivalent series RLC circuit in parallel with the intrinsic TF capacitance, and then excluding the effect of the inherent TF capacitance. Figures 7.2b and 7.2c present the resulting spectra of the TF as a mechanical damped oscillator and the simultaneous acoustic measurements, respectively. Resonance frequencies (colored dotted lines in Figures 7.2) determined using acoustic curves is consistent those shown in converted TF spectra, whereas resonance peaks obtained from directly measured TF curves are slight different. 

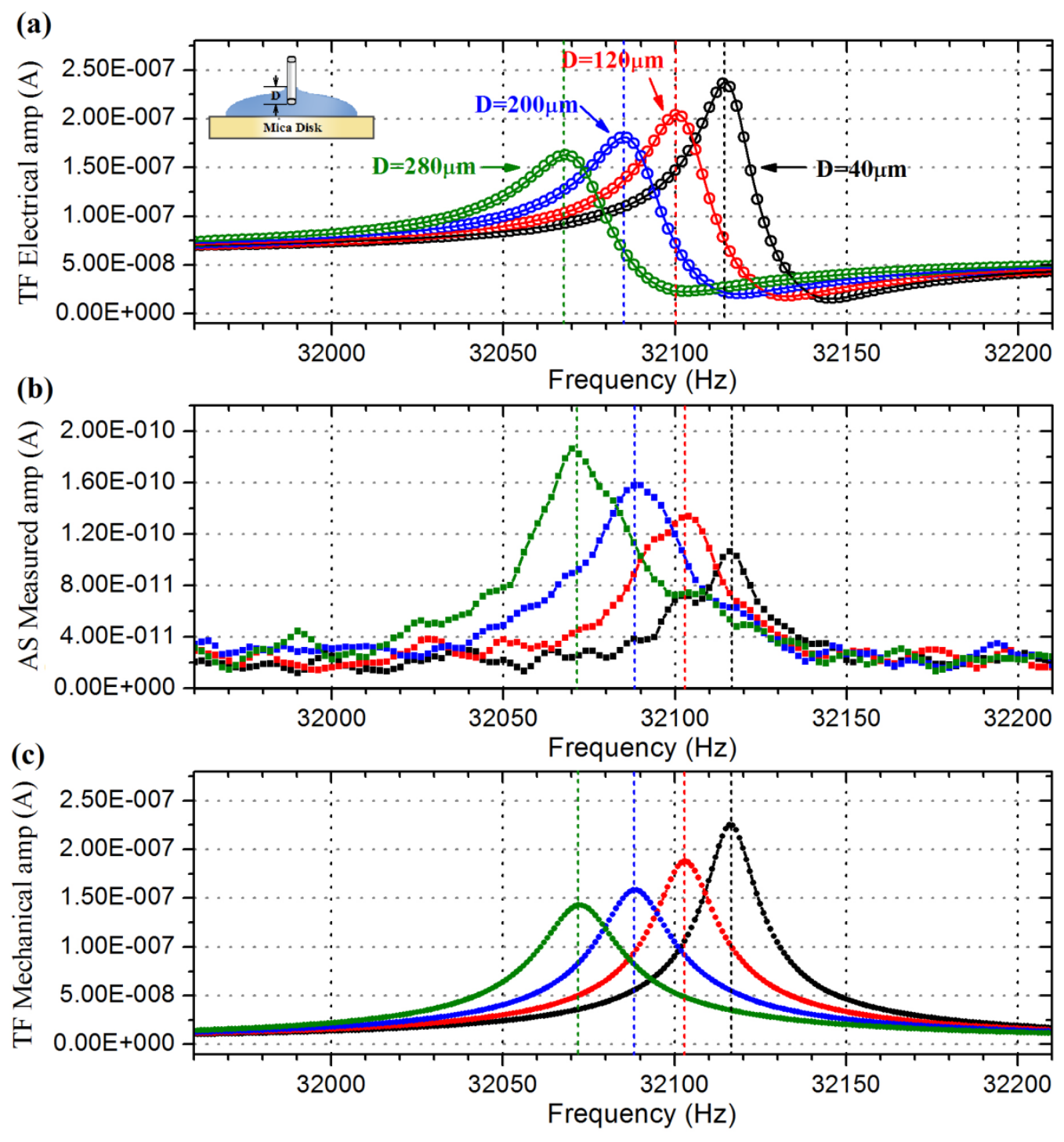

Figure 7.2: Typical vibration spectra of the TF (a) and acoustic (b) signals at immersion depth $=40 \mu \mathrm{m}$ (black), $120 \mu \mathrm{m}$ (red), $200 \mu \mathrm{m}$ (blue) and $280 \mu \mathrm{m}$ (green), as well as the converted TF spectra which represent the mechanical behavior of a harmonic oscillator (c).

With the increase of fiber immersion depth, a negative shift of resonance frequency and a change in oscillation amplitude are observed in both TF and acoustic measurements. We plot the variations in frequency and amplitude as a function of penetration depth under different experimental conditions, such as using glycerol aqueous solutions with 
various viscosities as the bulk liquid, and fibers with different diameters as the probe. Our goal is to clarify the effects of liquid viscosity and probe diameter in shear-force and acoustic detections.

\subsubsection{Effects of Fluid Viscosity and Probe Diameter in Frequency Shift}

Figures 7.3 and 7.4 demonstrate the negative frequency shift in the resonance positions of TF and acoustic vibration spectra as a function of penetration depth. Our measurements were performed at the same experimental conditions (i.e., excitation voltage of TF, settings of lock-in amplifiers, temperature and environmental humidity) but using the same probe in glycerol-water solutions with different viscosities (Figure 7.3), or using fiber probes with various diameters in the same liquid (Figure 7.4). As expected, when the TF is immersed into liquid, it undergoes a remarkable resonance

frequency shift (towards lower frequencies) due to the external mass loading ${ }^{117}$. As the penetration depth of the fiber probe increases, its surface area exposed to the liquid becomes larger. As a result, the mass loading applied to the fiber increases, giving rise to the corresponding negative resonance frequency shift. We also note that the resonance peaks of acoustic signal tend to shift towards the same direction with the same amount as that of converted TF signal, whereas the direct TF measurements exhibit a larger frequency shift than the converted spectra (not shown on the figure). 
Frequency Shift using Fluids with Different Viscosities
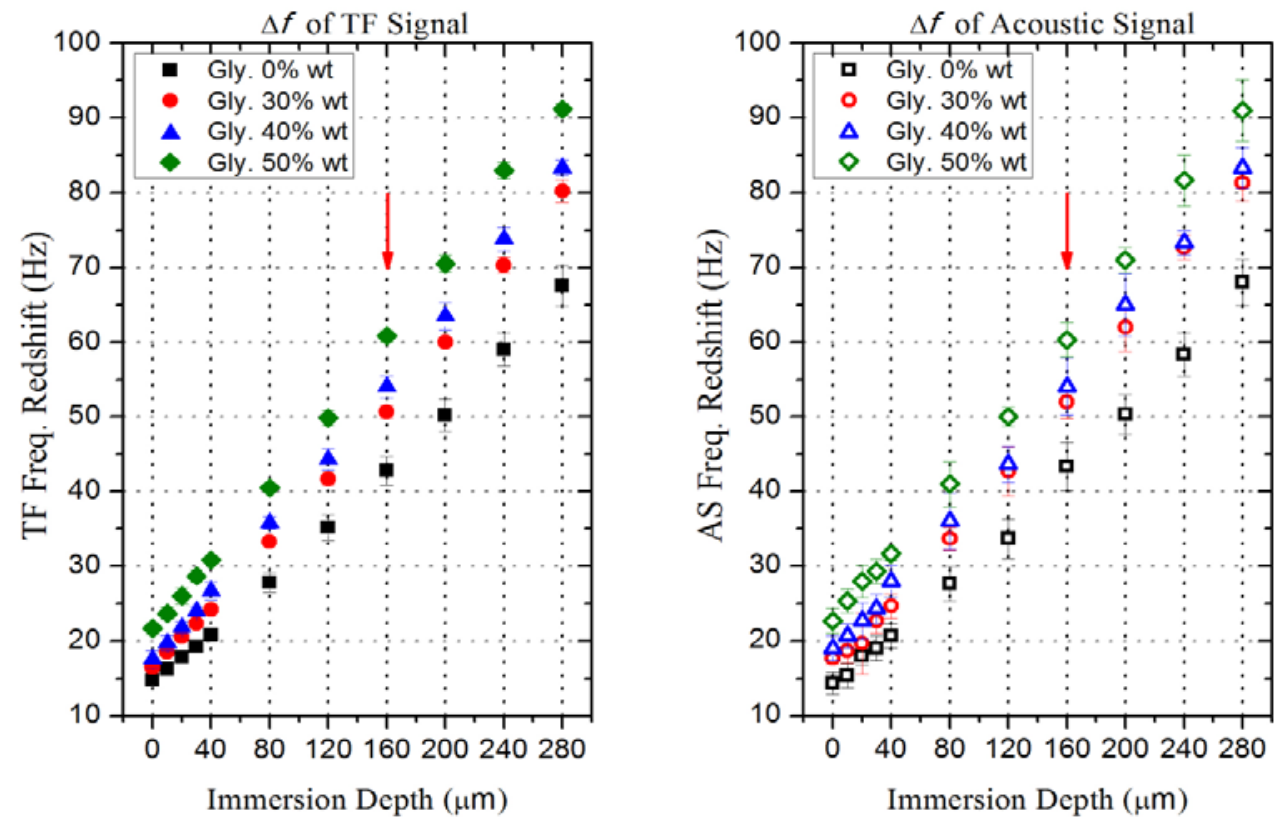

Figure 7.3: Resonance frequency shift of the TF (left) and acoustic (right) signals as a function of penetration depth using the same probe in liquids with different viscosities.
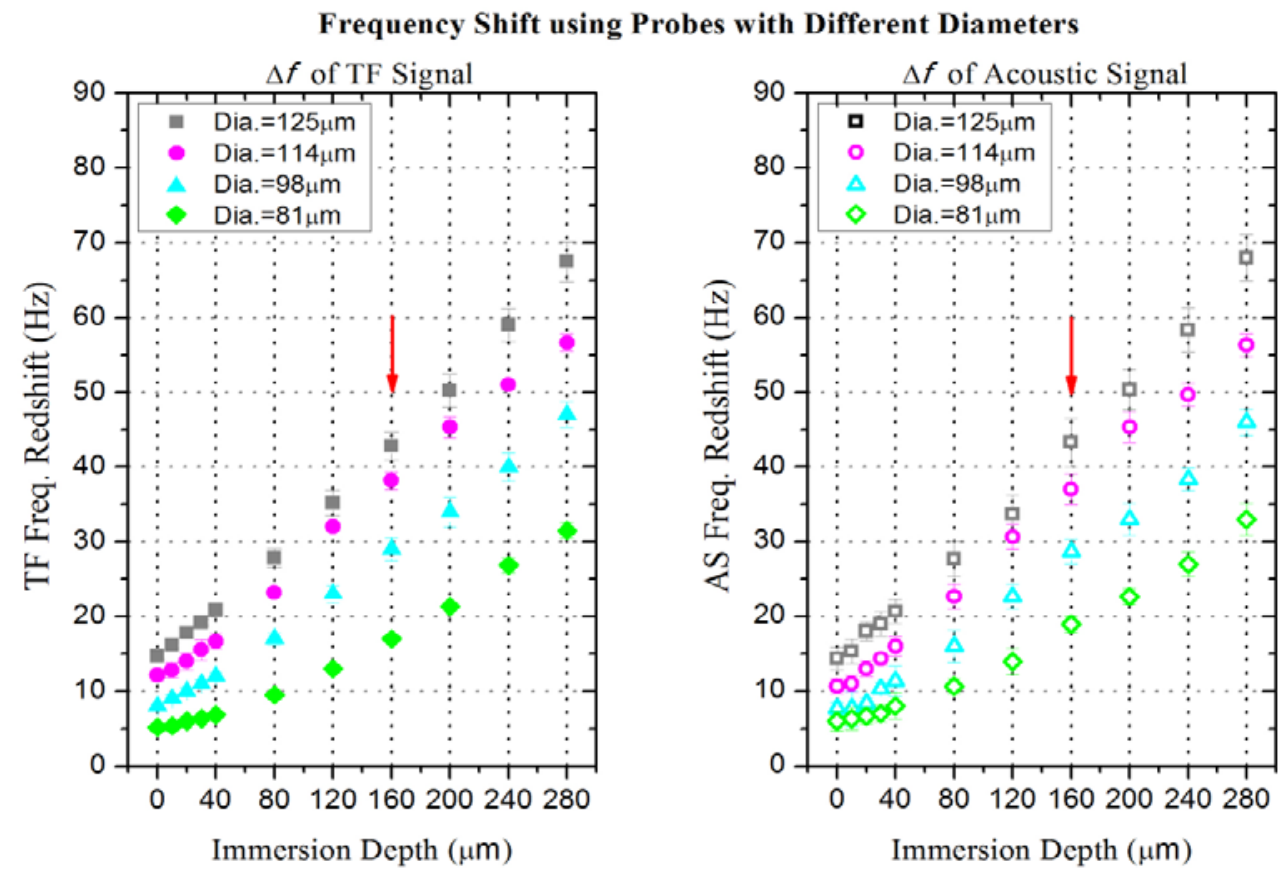

Figure 7.4: Resonance frequency shift of the TF (left) and acoustic (right) signals as a function of penetration depth using probes with various diameters in the same liquid. 
At a fixed immersion depth (i.e., $160 \mu \mathrm{m}$ ), the influence of probe diameter in resonance frequency shift is noticeable compared to that of fluid viscosity. The reason is that an increase in the diameter of fiber probe leads to a larger surface area in contact with liquid, and consequently results in an increase of the mass loading. However, when the same probe is immersed in solutions with different viscosities, the added mass may not be significantly changed because the mass densities of solutions are similar ${ }^{115}$.

\subsubsection{Effects of Fluid Viscosity and Probe Diameter in Amplitude Variation}

In application of the acoustic method, our concern is with the impacts of liquid viscosity and probe diameter on the amplitude of measured signals. Figures 7.5 and 7.6 show the change of signal amplitude in the resonance positions of TF and acoustic spectra at various immersion depths. We carried out the same experiments for increasing penetration depth as mentioned above (in different solutions and using different probes, respectively). Under the monitoring of a CCD camera, we observed once the fiber probe enters the water film (depth $=0$ ) the TF amplitude decreases abruptly. This sudden fall in TF signal corresponds to the situation in which the liquid meniscus is formed surrounding the fiber. The similar behavior found in all TF detection is that the amplitude reduces as the immersion depth increases. In contrast, the amplitude of acoustic signal increases when the fiber penetrates deeper into the liquid. The possible reason is due to the viscous drag force acting on the fiber vibrating inside the liquid. The deeper is the fiber in fluid, the larger is the viscous drag experienced by the fiber. In addition, the liquid is moving back and forth due to the dipping fiber. The motion of the fluid, together with the probe 
has contribution to producing acoustic waves. The more liquid is dragged by the fiber, the more sound is created and detected by the acoustic sensor. It is of relevant importance to point out that data of acoustic signal has been processed. We divided the measured acoustic amplitude by the corresponding TF amplitude so that normalized data points shown in the right panel of Figures 7.5 and 7.6 represent the intensity of sound created when driving the probe to oscillate at the same amplitude.
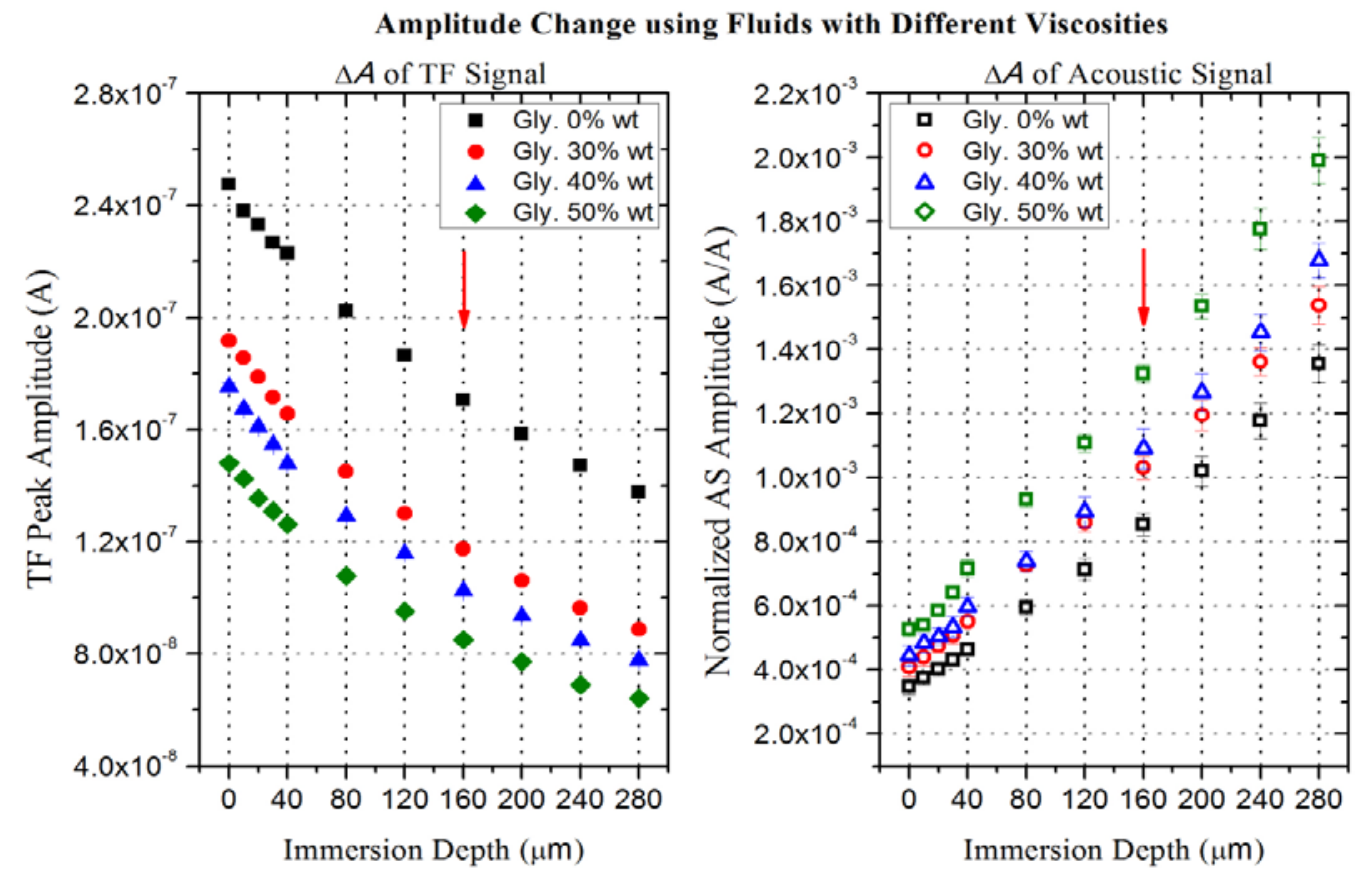

Figure 7.5: Change in amplitude of resonance peaks of the TF (left) and acoustic (right) signals as a function of penetration depth using the same probe in liquids with different viscosities. 

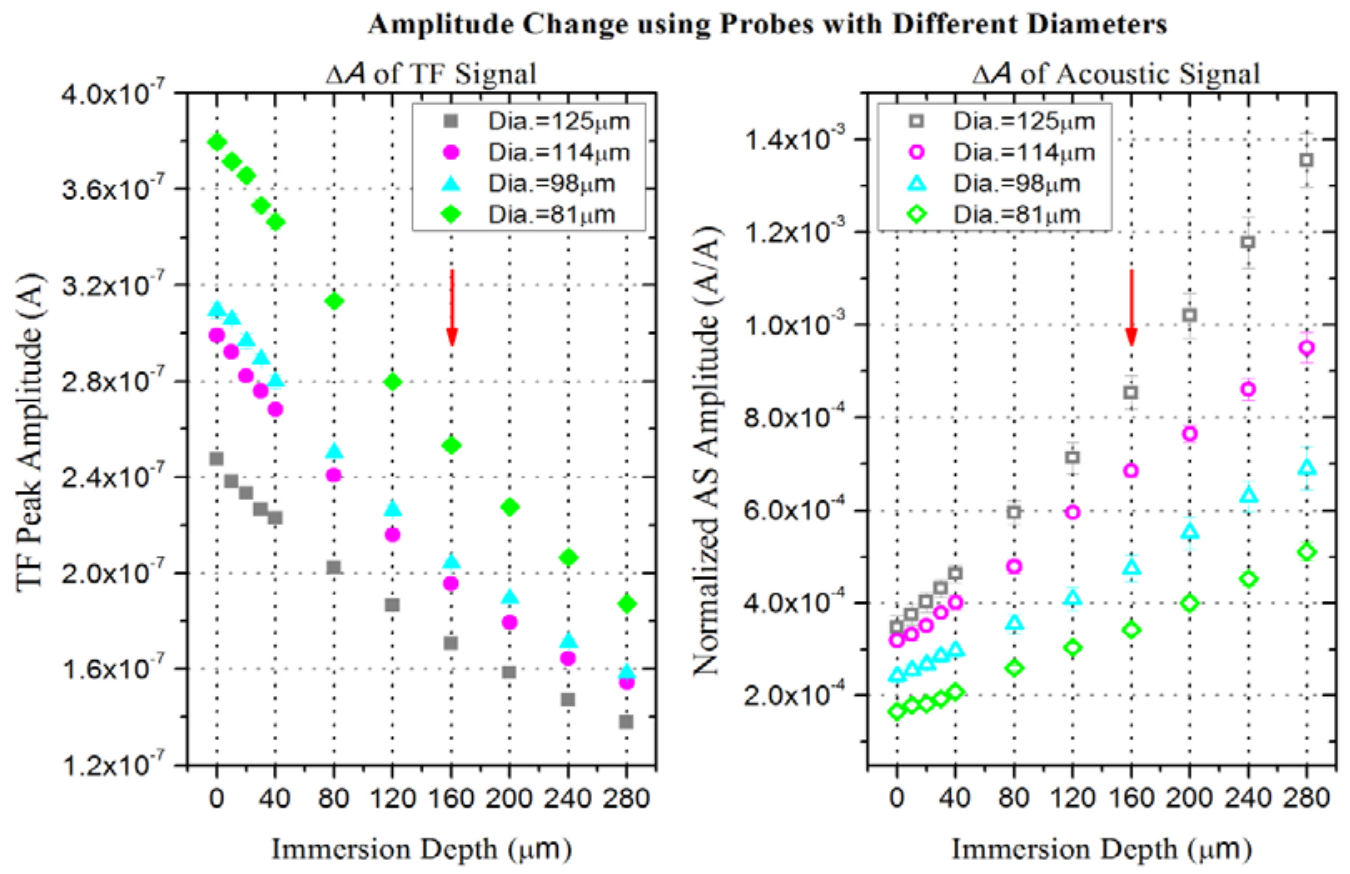

Figure 7.6: Change in amplitude of resonance peaks of the TF (left) and acoustic (right) spectra at various penetration depths using probes with different diameters in the same liquid.

At a constant immersion depth (i.e., $160 \mu \mathrm{m}$ ), we observed the acoustic signal is enhanced by either dipping the probe into more viscous liquid or using the fiber with larger diameter. This result is consistent with our expectation that the generation of acoustic wave is dependent on how the probe interacts with the liquid. An increase in both fluid viscosity and liquid-probe contact area helps produce more sound.

\subsubsection{Influences of Other Factors in Shear-Force and Acoustic Measurements}

\section{Case 1: The Effect of Applied Driving Voltage}

Figure 7.7 demonstrates the case in which the same fiber probe is excited to laterally oscillate in distilled water at different immersion depths (20 $\mu \mathrm{m}, 40 \mu \mathrm{m}, 80 \mu \mathrm{m}$ and 160 
$\mu \mathrm{m}$ ). We observed that the obtained TF (top) and acoustic signals (middle) have a good linear relationship with respect to the driving voltage. However, the normalized acoustic signal is independent of exciting voltage (bottom). The result suggests that at a fixed depth, the intensity of acoustic wave generated due to the same amount of probe oscillation is not influenced by the voltage applied to the TF. This offers benefits in our experiments, because the relatively high driving voltage leads to the increased signal-tonoise ratio and makes the accurate measurement easy.
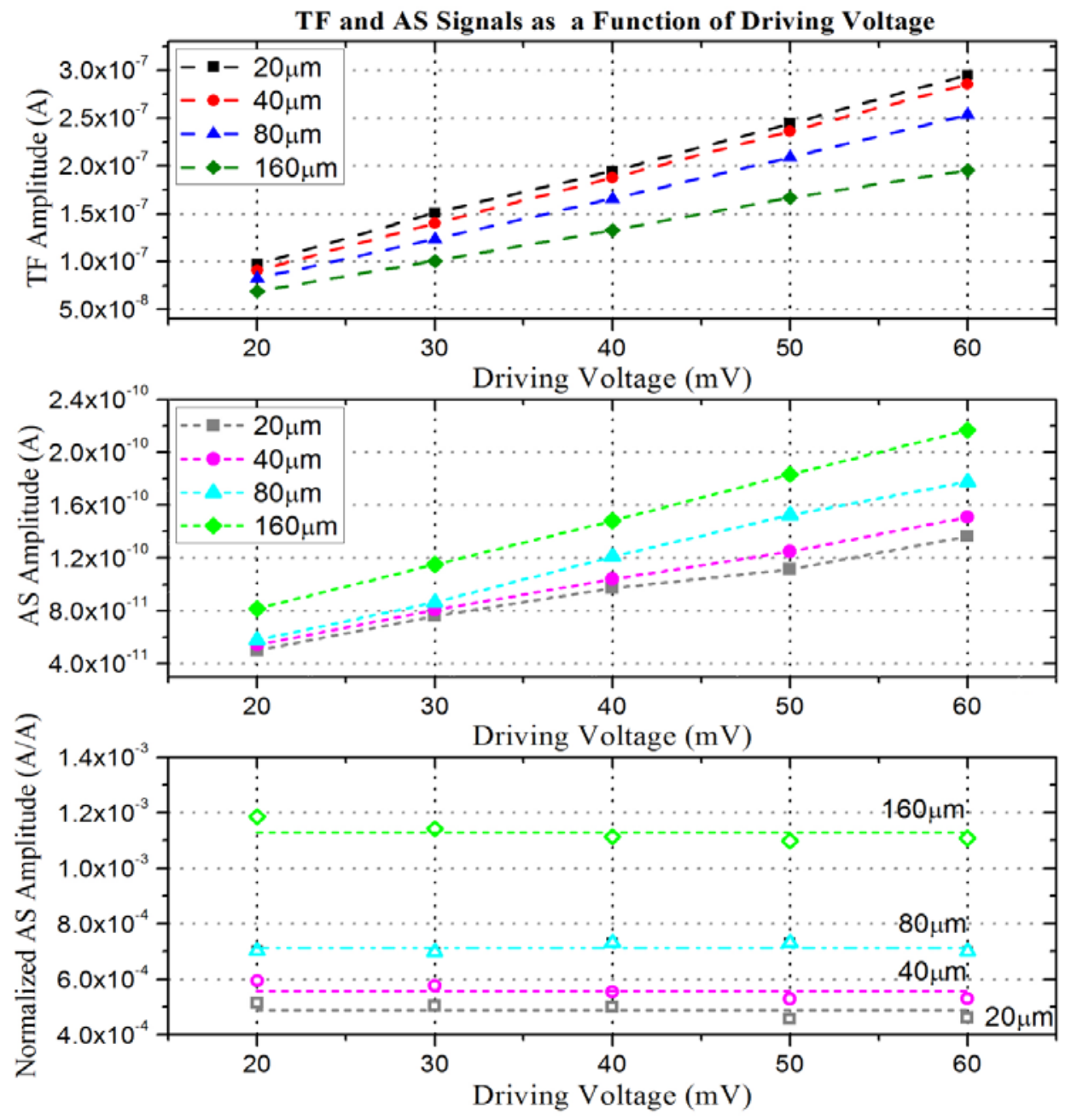

Figure 7.7: Plots of measured amplitude of the TF (top) and acoustic (middle) signals at resonance as a function of driving voltage, at different submersion depths, respectively. 
However, the normalized acoustic amplitude is independent of the driving voltage (bottom).

\section{Case 2: The Effect of Liquid Droplet Volume}

Our previous measurements were performed by submerging the probe into liquid droplets with the same volume ( $5 \mu \mathrm{L}$ ). Figure 7.8 demonstrates the case in which the same fiber laterally oscillates in droplets of distilled water with different volumes ( $5 \mu \mathrm{L}$, $7.5 \mu \mathrm{L}, 10 \mu \mathrm{L} .12 .5 \mu \mathrm{L}$ and $15 \mu \mathrm{L}$ ). We chose the same penetration depth (80 $\mu \mathrm{m})$ when carrying out this experiment. However, the distances from the end of fiber to the acoustic sensor are varied because of the change in liquid volume. In the figure, the obtained curves are overlapped, suggesting that the size of liquid droplet does not affect our experimental results. A little variation of fiber-sensor distance does not have any impact on the acoustic detection. 
TF and AS Signals at Depth $=\mathbf{8 0} \mu \mathrm{m}$ in Drops with Different Volume
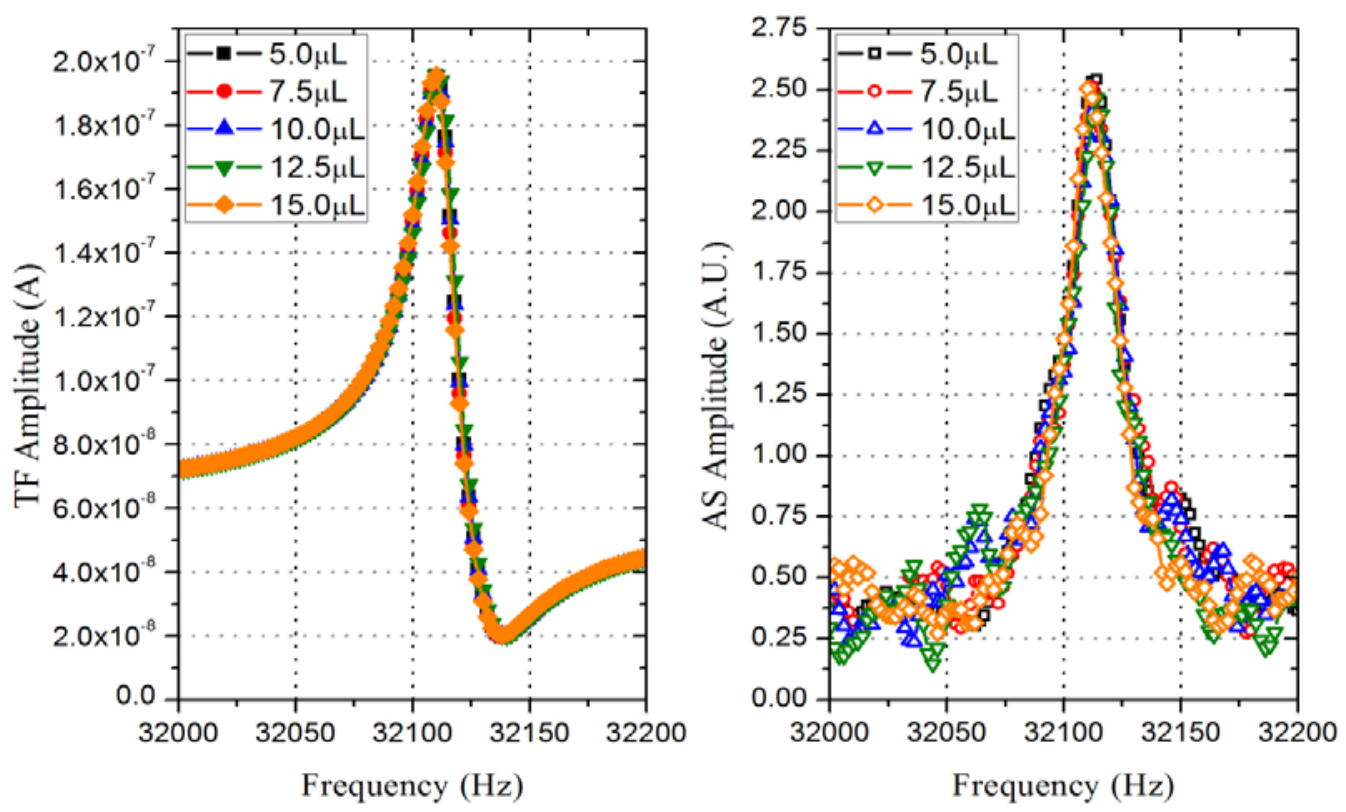

Figure 7.8: Vibration spectra of the TF and acoustic signals obtained by exciting the same fiber to oscillate in drops of distilled water with different volumes.

\section{Case 3: The Effect of Liquid Evaporation during Experiments}

The evaporation of liquid would be a factor that may affect the measured signals. As mentioned above, we observed a negative frequency shift at resonance position on obtained curves when the probe is immersed into bulk liquid. As the drop of liquid evaporates during our experiment, however, the amount of liquid in contact with the probe decreases, resulting in a shift of the resonance frequency towards higher frequencies, which is in opposition to our expectation. To evaluate the effect of liquid evaporation, we recorded the vibration spectra of TF and acoustic signals at a constant depth of $80 \mu \mathrm{m}$ in distilled water. In Figure 7.9, the time interval between two traces next to each other is 1 minute. The entire recording lasted $\sim 5$ minutes, which is much longer 
than the average time of experiments described above. The frequency shift due to the evaporation is not detectable; therefore we can neglect its influence in our measurements.

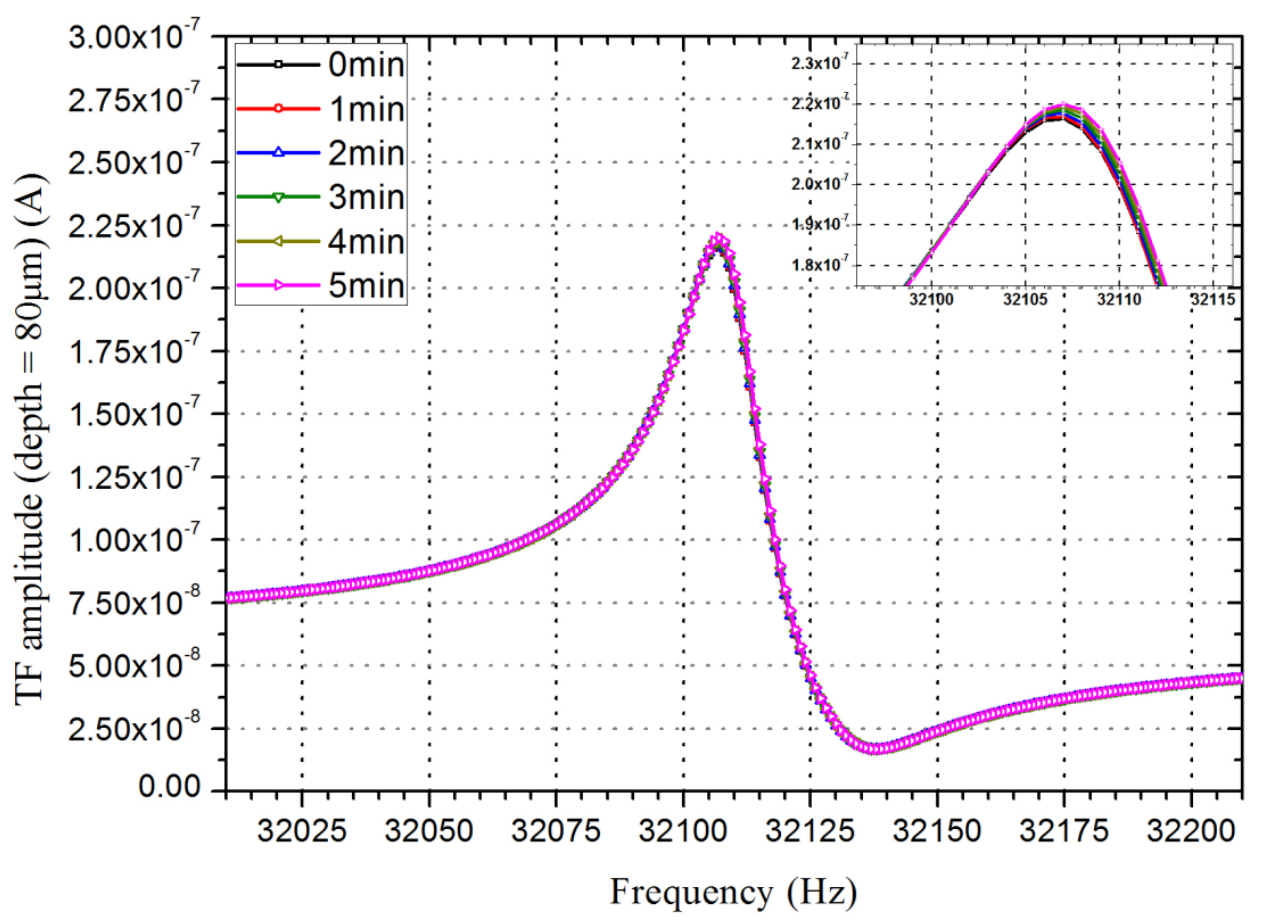

Figure 7.9: Recording TF frequency response when the fiber oscillates and stays at a fixed depth in distilled water. The time interval between two traces next to each other is 1 min, and the entire process lasts $\sim 5 \mathrm{~min}$. The details of resonance position are shown in the inset.

\subsection{Conclusions}

Compared to the measured TF signal, the acoustic probing technique is able to reflect the actual mechanical behavior of the probe in liquid while the TF is electrically driven. A cylindrical fiber served as the probe that was mounted on one of TF prongs. We carried out a series of experiments, which are analogous to the situation at mesoscopic scale, by dipping the protruding portion of the fiber into a drop of liquid. We changed the fluid 
viscosity by preparing glycerol aqueous solutions with various glycerol concentrations. Fiber probe can also be thinned via chemical etching. We recorded the vibration spectra of TF and acoustic signals, and observed a red-shift of resonance frequency as the increase in immersion depth. It is because the external mass loading applied to the oscillating element when penetrating into liquid. We also found that the measured TF signal underwent a remarkable damping, whereas the amplitude of the acoustic signal (normalized) at resonance increases when the fiber was immersed in liquid. Using the same fiber and the same solution, the deeper the fiber is in solution, the larger the viscous drag is experienced by the fiber, and the higher the acoustic signal is detected. At a constant depth, either dipping the probe into more viscous liquid or using the fiber with larger diameter helped produce more sound. Finally, we discussed effects of other factors, such as driving voltage, drop size and liquid evaporation. Experimental results showed that they did not play any important role in our measurements. 


\section{Chapter 8. Detection of Carbohydrate-Protein Interactions via Shear- Force/Acoustic Near-Field Microscope}

\subsection{Introduction}

Carbohydrates are the most abundant biomolecules in nature and essential elements in a wide range of processes in living systems. Aside from their use as structural materials and energy sources, they are to large extents mediating recognition events through their interactions with proteins and other biological entities. Molecular recognition events of carbohydrates and their protein pairs are involved in many important biological processes,

mediating cell behaviors in numerous diseases including cancers ${ }^{118-124}$. Thus probing carbohydrate-protein interactions becomes the prerequisite to gain insight into the physical and chemical details of cellular events, to develop new bioanalytical and diagnostic applications.

Direct detection of interactions between specific ligands (carbohydrates) and receptors (proteins) at the molecular level attracts considerable interest from both theoretical and practical points of view. Interactions of carbohydrate-protein pairs, both as free ligands in solution, have been investigated by a variety of biochemical and biophysical solutions, such as NMR spectroscopy ${ }^{125}$, SPR spectroscopy ${ }^{126,127}$, X-ray crystallography $^{128,129}$, titration microcalorimetry ${ }^{130}$, and fluorescence spectroscopy ${ }^{131}$. Furthermore, the emergence and development of Single-Molecule Force Spectroscopy (SMFS) techniques including atomic force microscope (AFM), laser optical tweezers (LOT), magnetic tweezers (MT), biomembrane force probe (BFP) and single-molecule fluorescence (SMF) have opened up a wide range of novel opportunities to explore 
biomolecular interactions of ligand-receptor complexes, in real time, under conditions which are easily manipulated ${ }^{132,133}$. SMFS techniques are considered to be well suitable for measuring the strength of the interaction forces and investigating the thermodynamics and kinetics of biomolecular processes. The AFM, as one of the most powerful and established SMFS, has now advanced beyond use as an imaging tool, allowing one to measure the binding forces with extremely high sensitivity as piconewton and the length changes with exquisite spatial resolution as Ångström ${ }^{134}$. However, when AFM measuring is carried out, only the forces acting on the tip can be picked up, information with respect to the substrate in response to the binding formation or breakage is difficult to acquire ${ }^{135}$. Therefore new versatile methodology which can provide detailed interpretation of carbohydrate-protein interactions at molecular level through both aspects of the probe and the sample still represents a considerable challenge.

We developed a new technique named the shear-force/acoustic near-field microscopy ${ }^{63,73}$, which mainly consists of a quartz tuning fork (TF) with a tapered tip mounted on one of tines, and an acoustic sensor (AS) located under a sample substrate. We initially aim to use it for investigating dynamics of fluid-like contamination layers on solid surfaces at mesoscopic scale under ambient conditions. It is believed that the presence of the mesoscopic fluid film is a potential mechanism to explain the shear-force effect, which has been employed to control the probe's vertical position relative to the sample in near-field scanning probe microscopy. Herein, our goal is to demonstrate the sensing capability of this shear-force/acoustic based technique to probe carbohydrateprotein interactions. By integrating the acoustic sensor into the conventional shear-force 
microscope, we expect to detect binding and/or unbinding of specific carbohydrateprotein complex, particularly, in a form of acoustic information.

Carbohydrate ligands were covalently attached to the apex of the tip, and correspondingly, proteins were immobilized on the substrate. Both the tip and the substrate were functionalized using a unique photochemical coupling method based on perfluorophenyl azide (PFPA), which has become popular for conjugating polymers, small molecules and nanoparticles on various types of material surfaces ${ }^{136-139}$. This surface modification method relies on the photochemistry of arylazides: when exposed to ultraviolet (UV) light irradiation, the azides become highly reactive singlet nitrene moieties that fabricate robust covalent attachment by inserting into $\mathrm{CH}$ and $\mathrm{NH}$ bonds ${ }^{140,141}$. Recently, PFPA-functionalized carbohydrate was employed to fabricate glycan microarray, and surface-tethered ligands retained biological activity to their binding proteins ${ }^{142,143}$. Concanavalin A (Con A), extracted from jack bean, has been well studied and widely used because of its specific affinity to $\alpha$-D-mannopyranoside, $\alpha$-Dglucopyranoside, and their derivatives ${ }^{144,145}$. In our study, the binding site of Con A with mannose is employed as an example for a qualitative study of the recognition process. Information about the interaction between Con A and mannose molecules will be extracted from simultaneous measurements of the TF and acoustic signals with a change in the distance between the mannose-terminated probe and the Con A-terminated substrate. Additionally, control experiments using the same shear-force/acoustic microscope were performed on nonspecific binding surfaces to examine the selectivity of carbohydrate-protein interactions. 


\subsection{Experimental Section}

As shown in Figure 8.1, the left panel demonstrates the experimental procedure to covalently tether mannose molecules to a gold tip. It included four main steps: (i) Fabrication of sharpened gold tips which can be easily done via electrochemical etching in a concentrated $\mathrm{HCl}$ solution ${ }^{65}$; (ii) PFPA-disulfide coating which introduces PFPA self-assembled monolayers (SAMs) on gold tip surface; (iii) UV-induced poly(ethylene oxide) (PEO) spacer attachment that can significantly reduce nonspecific protein binding $^{146}$; and finally (iv) synthesized PFPA-mannose immobilization onto the PEO polymer under UV activation. The right panel illustrates the main steps involved in the process for protein molecules being covalently immobilized onto a flat silicon wafer. Two types of proteins, Con A and peanut agglutinin (PNA) were respectively attached onto each half of the same silicon substrate through a similar coupling method to that used in the preparation of carbohydrate-terminated tips as long as replacing PFPAdisulfide by PFPA-silane. Therefore, this versatile PFPA photochemistry has been applied totally three times in different ways but with the same aim: creating covalent linkages via PFPA groups to attach desired molecules on either tips or substrates. Experimental details regarding the probe and sample preparation processes are given in the following two paragraphs. 

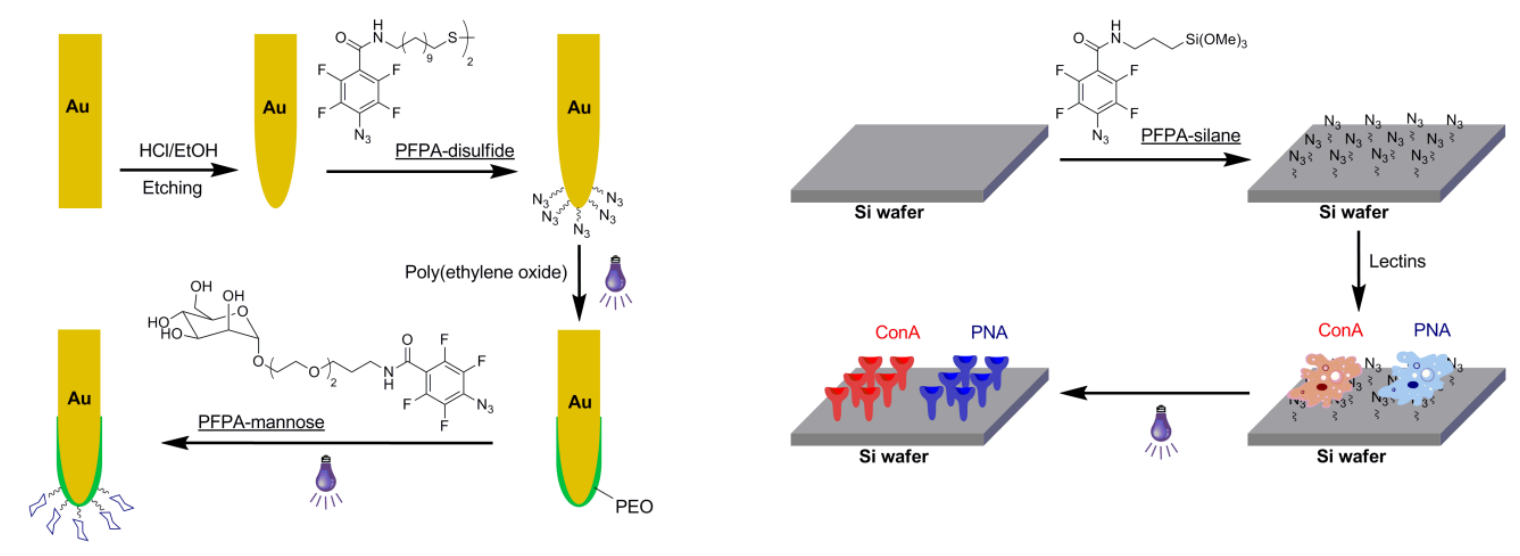

Figure 8.1: Schematic of experimental procedure to prepare the mannose-terminated gold tip (left) and the protein-immobilized silicon wafer (right).

Preparation of carbohydrate-coated gold tip: The gold wire of $76 \mu \mathrm{m}$ diameter (Au, purity 99.95\%, ESPI) was etched in a mixture of concentrated $\mathrm{HCl}$ acid and ethanol solution (1:1, v/v), and the obtained gold tip was rinsed with distilled water and characterized using optical and scanning electron microscopy (SEM). The tip was immersed in ethanol solution of PFPA-disulfide ${ }^{139}$ (20 mM) overnight and cleaned 5 times with pure ethanol. Subsequently a layer of poly(ethylene oxide) (PEO, MW 200,000, Aldrich) was coated using a well-established approach ${ }^{99,141}$ : the PFPAfunctionalized tip was immersed in aqueous PEO solution (10 mg/mL) for 1 hour and then irradiated with $280 \mathrm{~nm}$ optical filter under 450W medium-pressure Hg lamp (Hanovia) for $30 \mathrm{~min}$. The excessive polymer on the tip was removed by ultrasonicating and rinsing with water for 5 times. The carbohydrate was coated following a similar PFPA-based photochemical strategy: PFPA-derivatized mannose (PFPA-mannose) was synthesized via a published procedure in a previous report ${ }^{143}$, and the PEO-coated tip was irradiated under the same condition for 15 min after immersing in aqueous solution of 
PFPA-mannose ( $1 \mathrm{mg} / \mathrm{mL})$ for 1 hour. The tip was cleaned afterward in water 5 times and dried with nitrogen.

Preparation of protein-immobilized silicon substrate: The $1 \mathrm{x} 1 \mathrm{~cm}$ silicon wafers with a native $\sim 35 \AA$ thick oxide layer were cut with a diamond pen and cleaned initially in piranha solution (7:3 v/v conc. $\mathrm{H}_{2} \mathrm{SO}_{4} / \mathrm{H}_{2} \mathrm{O}_{2}$ ) for 2 hour at $80-90{ }^{\circ} \mathrm{C}$, washed thoroughly with boiling water for 1 hour, and dried under nitrogen. Cleaned wafers were soaked in toluene solution of PFPA-silane ${ }^{136}(12.6 \mathrm{mM})$ for 4 hour, rinsed in toluene 5 times, and dried under nitrogen. These treated wafers were then allowed to cure at room temperature for at least 24 hour before use. Solutions of Concanavalin A (Con A) and Peanut Agglutinin (PNA) (1mg/mL) were prepared in $\mathrm{pH} 7.4 \mathrm{PBS}$ buffer (10mM, 0.137 $\mathrm{M} \mathrm{Na}^{+}$, $1 \mathrm{mM} \mathrm{Ca}^{2+}$ and $1 \mathrm{mM} \mathrm{Mn}^{2+}$ ) and $50 \mu \mathrm{L}$ of each solution was dropped respectively onto different areas of the PFPA-decorated wafer. Protein immobilization was also carried out in similar PFPA-based fashion described above: the wafer with protein drops were irradiated immediately with optical filter for $7 \mathrm{~min}$, and unbounded proteins were rinsed off with PBS buffer.

The experimental setup used for synchronously detecting the shear-force and the acoustic responses from the probe and the sample is schematically presented in Figure 8.2. Briefly speaking, as shown in Figure 8.2(left), the core of the shear-force/acoustic microscope consists of (i) a piezoelectric tuning fork with a tapered gold tip on which mannose was covalently tethered; and (ii) an acoustic transducer placed under a silicon substrate on which protein molecules were covalently attached. In details, as demonstrated in Figure 8.2(right), the entire experimental system can be broken down 
into two distinct sections: the microscope stage and the detection system. The microscope stage sits on a floating optical bench to minimize mechanical perturbations. A piece of rubber cushion is placed between the stage and the optical bench to further reduce undesigned disturbances. Under monitoring of a CCD camera, smoothe movement of the probe in the perpendicular direction to the sample surface ( $\mathrm{Z}$ axis) is accomplished through coarse and fine adjustments of the probe-sample distance. Coarse control is performed using a set of fine-pitch screws (Newport; model: AJS100-2, travel: $50.8 \mathrm{~mm}$, sensitivity: $0.71 \mu \mathrm{m}$ ), which are able to provide a rapid movement of a few milimeters with precision in the order of micrometer. Once the probe-sample distance falls into the range of $50 \mu \mathrm{m}$, fine control is activated using a piezo-actuated nanopositioner (NanoOP65, Mad City Labs, Inc.), which is capable of moving the tip in a range of $65 \mu \mathrm{m}$ with nanometer accuracy. More importantly, this nanopositioner can be operated in close-loop mode so that hysteresis arisen from the piezoelectric components can be completely compensated. 

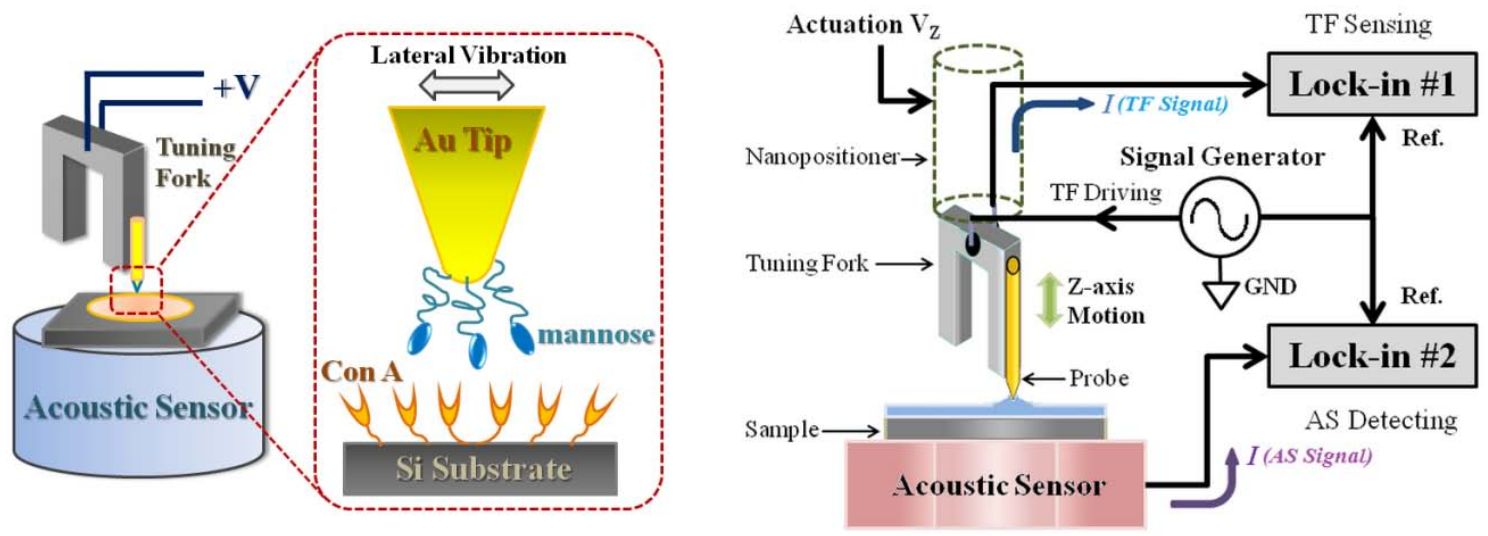

Figure 8.2: Schematic of experimental setup used to detect the TF signal along with the acoustic signal as the gold tip approaches towards and subsquently retracts from the sample surface.

In actual operation, a quartz tuning fork (TF) is electrically excited at its resonance frequency by applying AC voltage through its electrodes. Thus its tines where the functionalized gold tip is attached to one of them oscillate in a direction parallel to the sample surface. Both actuation of the TF and detection of its response are achieved using the same lock-in amplifier (Lock-in \#1 shown in Figure 8.2(right), SR850 model, Stanford Research System). Selection of the TF excitation voltage usually depends on the Q-factor of the TF being used. In our experiment, a driving voltage of $10 \mathrm{mV}_{\mathrm{rms}}$ is chosen; and approximately, the probe is driven to oscillate with an amplitude of $12 \mathrm{~nm}$ in air. The measurement of an electric signal from the acoustic transducer located under the sample is done by feeding the output of the sensor into the other lock-in amplifier (Lock-in \#2 shown in Figure 8.2(right), SR850 model, Stanford Research System). These two amplifiers share the same reference signal that is the excitation source of the TF. Furthermore, some parameters of these two lock-in amplifiers are set to be the same for 
convenient communication (i.e., a sample rate of $8 \mathrm{~Hz}$ and a time constant of $10 \mathrm{~ms}$ ). As the tip vertically moves toward/detaches from the sample surface, variations of the electric current produced by the TF and the acoustic sensor are recorded synchronously as a function of the probe-sample distance, and subsequently plotted as so-called approach/retraction curves. Generally, the velocity of the tip's movement is held constant at a low level (i.e., $1.5 \mathrm{~nm} / \mathrm{s}$ ) during both approach and retraction processes. The experiment was performed in an environment with relatively high humidity (R.H. was $\sim 70 \%)$.

\subsection{Results and Discussion}

In the first step, we were concentrated on the detection of interactions between Con A and mannose complexes, which is expected to be reflected from the measured approach/retraction curves. In one complete cycle, the mannose-terminated tip was first brought in close proximity to the modified surface from a position where it freely vibrated in air; and subsequently the tip was withdrawn from the sample until it fully returned to its initial oscillation amplitude. Both TF and acoustic electric signals were simultaneously collected while the tip-sample separation was varied with nanometer precision. Attempts were carried out on the Con A coated substrate. Experimental results shown in Figures 8.3 tend to demonstrate the capability of the shear-force/acoustic microscope for sensing Con A-mannose interactions. Approach and retraction curves (Figures 8.3a and 8.3b, respectively) are plotted to display variations of the TF and acoustic signals as a function of the probe’s vertical displacement. This manner is similar 
to a conventional AFM force-distance measurement. In our case, however, the vertical (Y) axis represent the measurement of electric current generated by the TF and the acoustic sensor due to their piezoelectric effect. For example, the amplitude of the TF signal is proportional to the amplitude of the TF's mechanical vibration. Differing from a direct measurement of the normal force acting on the tip using AFMs, the detected electrical signal which varies in response to the interplay between the tip and the surface can be considered as an indirect measurement of the probe-sample interaction forces.

Prior to start the approach/retraction operation, the frequency response of the TF in air was obtained in order to determine the base line of the TF signal. Therefore, when we plotted the resulting approach/retraction curves in Figure 8.3, the "zero" coordinates of the horizontal axis is arbitrarily defined as the position where the amplitude of TF signal drops by $95 \%$ of the difference between the maximum and the base line. However, the origin may not represent the actual location of the sample surface. As the mannosetethered tip approaches towards the Con A-coated surface, the TF and acoustic signals first remain constant until the probe's displacement reaches the position of $-9 \mathrm{~nm}$ on the horizontal axis (labeled by an arrow in Figures 8.3a). Afterwards, with the ongoing approach performance, the TF and acoustic signals start to change concurrently. We observed that the transition of the measured TF signal which is exhibited by a rapid fall in its amplitude from the top to almost the bottom of the curve takes place within a distance of $9 \mathrm{~nm}$ (from -9 $\mathrm{nm}$ to $0 \mathrm{~nm}$ on the horizontal axis). The decrease in the TF amplitude probably results from the shear-force effect caused by forces, such as electrostatic forces and a viscous drag experienced by the tip in a residual liquid layer ${ }^{73}$. Another potential 
mechanism that leads to attenuation of the probe's vibration is intermittent contact (tapping) of the tip’s apex with the surface at a short probe-sample distance. In contrast to monotonic decrease in the TF amplitude, the variation of the acoustic signal with the decrease in the probe-sample distance is not as simple as that shown on the TF curve: an increase of the acoustic signal above the background is first observed, then followed by a decrease, and finally ended with another trend of increasing the amplitude. Hypothesis was proposed to elucidate our observation of the acoustic measurement. For example, the initial increase in the acoustic amplitude with the decrease in the TF signal could be explained by an increased phase lagging between the vibrating probe and the mesoscopic fluid when the tip approached to the substrate: closer to the sample, the phase lagging become greater, and thereby sound generation is more efficient. In addition, at shorter probe-sample distance, the sharp reduction in the amplitude of tip oscillation makes the establishment and the preservation of mannose-Con A linkages possible. 

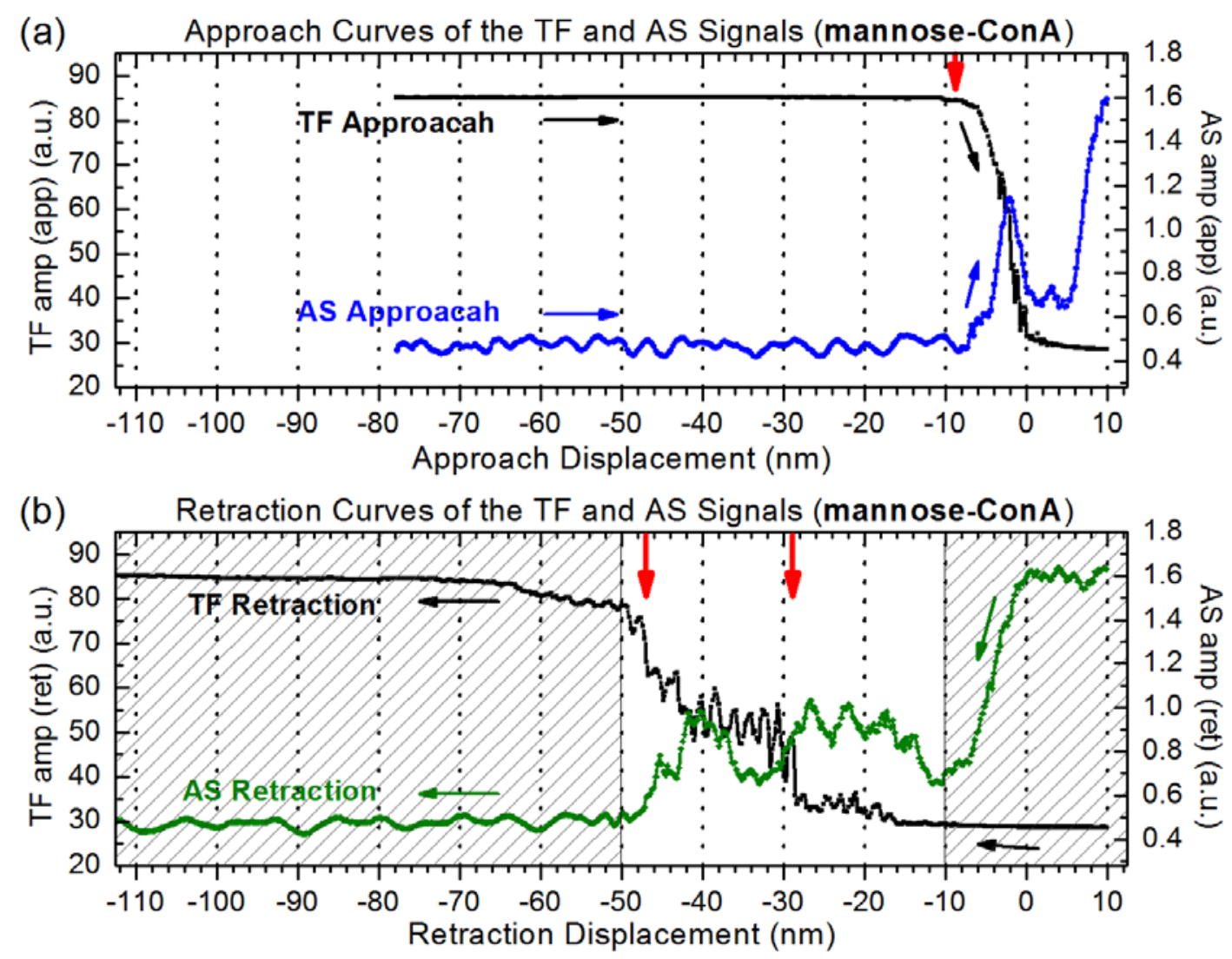

(c)

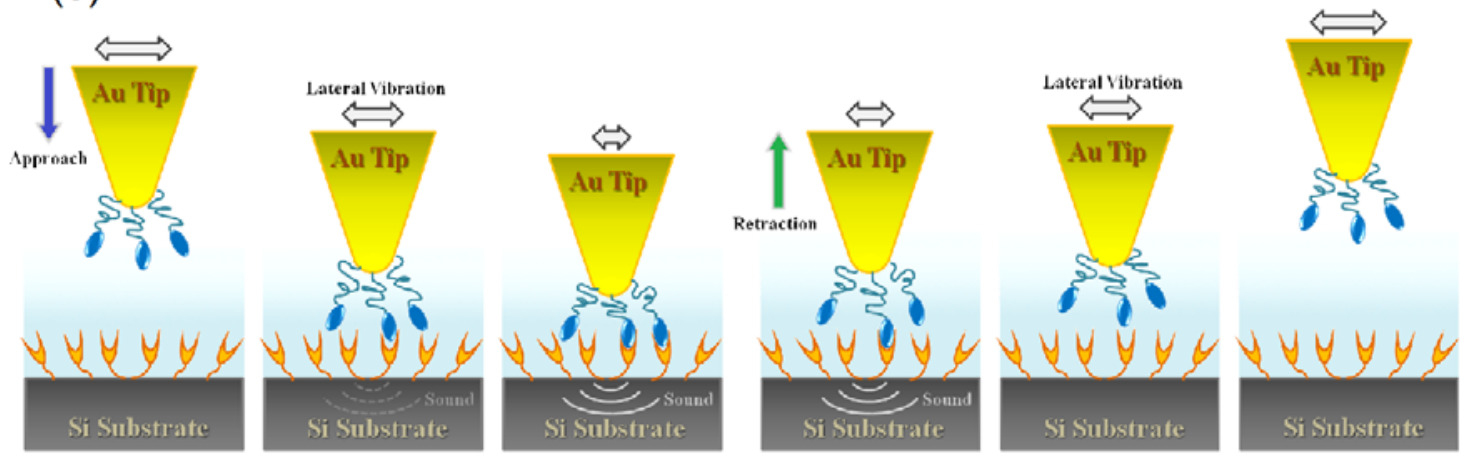

Figure 8.3: Typical approach (a) and retraction (b) curves of TF and acoustic signals plotted as a function of the tip's vertical displacement. (c) Schematic demonstration of the interaction between the mannose-terminated tip and the Con A-terminated surface.

We expect that, bonds between mannose and Con A molecules are formed in the region where the tip gets close enough to the surface (or, even they are in contact). As the 
tip detaches from the surface, rupture of those bonds occurs with increasing the probesample separation. Therefore, we are looking forward to probing unbinding of mannoseCon A pairs in subsequent tip retraction. In fact, similar to AFM force spectroscopy, bond rupture is more noticeable and easier to be detected than its formation. In our experiment, rupture events are likely presented by multiple peaks and valleys above the noise level, which are clearly visible on both the TF and acoustic retraction traces (Figure 8.3b). Comprehensive studies via dynamic force spectroscopy have reported the rupture force of single mannose-Con A bond is $50 \sim 200 \mathrm{pN}$ and binding energy is $16.6 \mathrm{~kJ} / \mathrm{mol}$, while the length of hydrogen bond between mannose and binding sites on Con A is around $5.3 \AA^{147-149}$. Herein, the key point that we highlight is to use the shearforce/acoustic technique for providing a qualitative impression of detachment of mannose from Con A, which so far has not been reported yet. In Figure 8.3b, we are more interested in the region approximately from $-10 \mathrm{~nm}$ to $-50 \mathrm{~nm}$ on the horizontal axis because of unique signals shown on both the TF and acoustic curves, which have not been present in our previous studies using the shear-force/acoustic microscope. For instance, the TF signal abruptly jumps up twice (marked by arrows in Figures 8.3b). It is possible that each jump corresponds to a sudden release from the surface due to rupture of bonds established between mannose and Con A molecules. Variations of the acoustic signal coincide with those of the TF measurement: specific signals above background noise are also detected by the acoustic sensor.

The mechanism of generation of sound waves in the mesoscopic layer was discussed in our previous studies. The acoustic signal detected by the bottom sensor is thought to be 
a dissipation channel of the probe's mechanical energy when the tip is laterally oscillating in the fluid film ${ }^{109}$. Biological interactions, particularly involving specific binding/unbinding events, are excellent platforms to test the capability of our acousticbased technique developed to investigate the probe-sample interplay in the near-field region. According to our previous studies, the measured TF and acoustic signals are greatly related to the probe-sample distance. During the retraction process, after the tip escapes from the adhesive surface (eliminating the effect of surface adhesion), the amplitude of the TF signal monotonic increases with increasing the gap between the tip and surface; whereas the amplitude of the acoustic signal generally decreases in the mesoscopic fluid film. However, owing to formation of bonds between the mannose and Con A molecules, a strong coupling between the tip and the sample is established (Figure 8.3c). It restricts the tip's movement, as evidenced by the plateau (in the unshadowed part of Figure 8.3b) on the TF curve. On the other hand, the enhanced coupling between the sample and the tip through chemical bonds is able to efficiently amplify the sound created due to the tip’s mechanical motion. Thereby the intensity of acoustic signal is still sufficient after separating the tip from the substrate by a relatively long distance. In addition, the protein molecules can also be dragged to laterally move by the vibrating tip, producing acoustic waves detected by the sensor attached to the bottom of the substrate.

The detection of bond rupture based on the shear-force/acoustic method is reproducible. Five retraction curves of the acoustic signal obtained by performing independent measurements under the same experimental conditions are shown in Figure 8.4. In comparison, they exhibit a similar characteristic: when the tip moves to the 
position of about $-40 \mathrm{~nm}$ on the horizontal axis, a specific peak above the background signal is consistently recognized on all acoustic traces. Based on this observation, most of rupture events occur at the certain displacement (energy level), in our case, it is at the location of $\sim 40 \mathrm{~nm}$ away from the surface. When the energy held on tip is high enough to overcome the energy barrier, the mannose-Con A complex will break apart, which is indicated by the appearance of specific peaks on the resulting curves. It is noticeable that the traveling distance of the tip to fully separate the mannose molecules apart from the Con A coated surface is much longer than the actual length of the hydrogen bond between the mannose-Con A pair. This observation is attributed to stretching of the PEO polymer layer that has been reported previously ${ }^{135}$. 


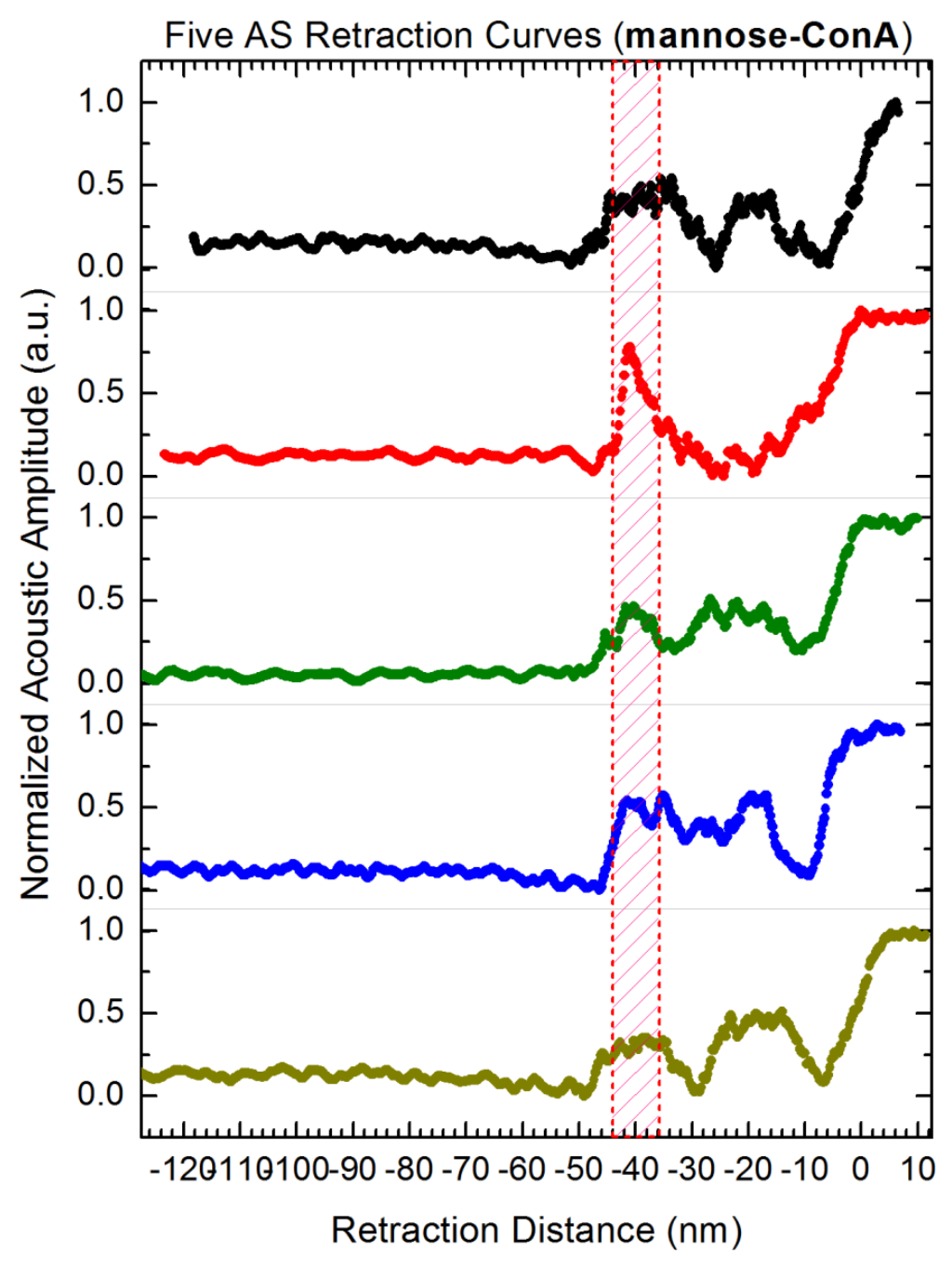

Figure 8.4: Repeatability of acoustic retraction traces representing mannose-Con A unbinding events.

As known in AFM measurements, surface adhesion could also give rise to peaks similar to those representing rupture events on force-distance curves. We may have the same problem when using the shear-force/acoustic microscope to study carbohydrateprotein interactions. In the second step, two control studies were carried out on unspecific binding surfaces to confirm that the appearance of unique peaks is due to unbinding of 
the mannose-Con A complex rather than surface adhesion. The same approach/retraction operation using the mannose-terminated tip was also performed on the surface covered by a monolayer of PFPA. The PFPA surface is not antifouling. It has considerable adhesion to biomolecules and always effectively reduces the sensitivity and obscure detection in sensing applications. For example, in a previous fluorescence microarray study, PFPAmodified surface exhibited significant nonspecific adsorption of lectins and thus the background noises ${ }^{150}$. However, as shown in Figures 8.5a (approach) and 8.5b (retraction), neither the TF measurement nor the acoustic detection gives specific peaks on their retraction curves in the region where rupture events are supposed to happen. In another control experiment, a different type of protein PNA that has specific affinity to galactose rather than mannose ${ }^{151}$ was deposited on the same substrate as was used for coating Con A. When the tip moves above the PNA coated side, there is no unique signal shown on the resulting curves as an indication of characteristic bindings/unbindings between the tip and the surface either (Figures 8.5c and 8.5d). The selectivity of our detection platform (the shear-force/acoustic microscope) is also examined on the PNAattached surface. Our results are consistent with a similar study that was carried out in a previous report using an AFM with a reverse binding geometry, where the protein was coated on the AFM tip and the substrate was functionalized with carbohydrates. Force curves were measured by the AFM; those displaying rupture peaks clearly represented the existence of specific interactions between mannose and Con A molecules ${ }^{147}$. 

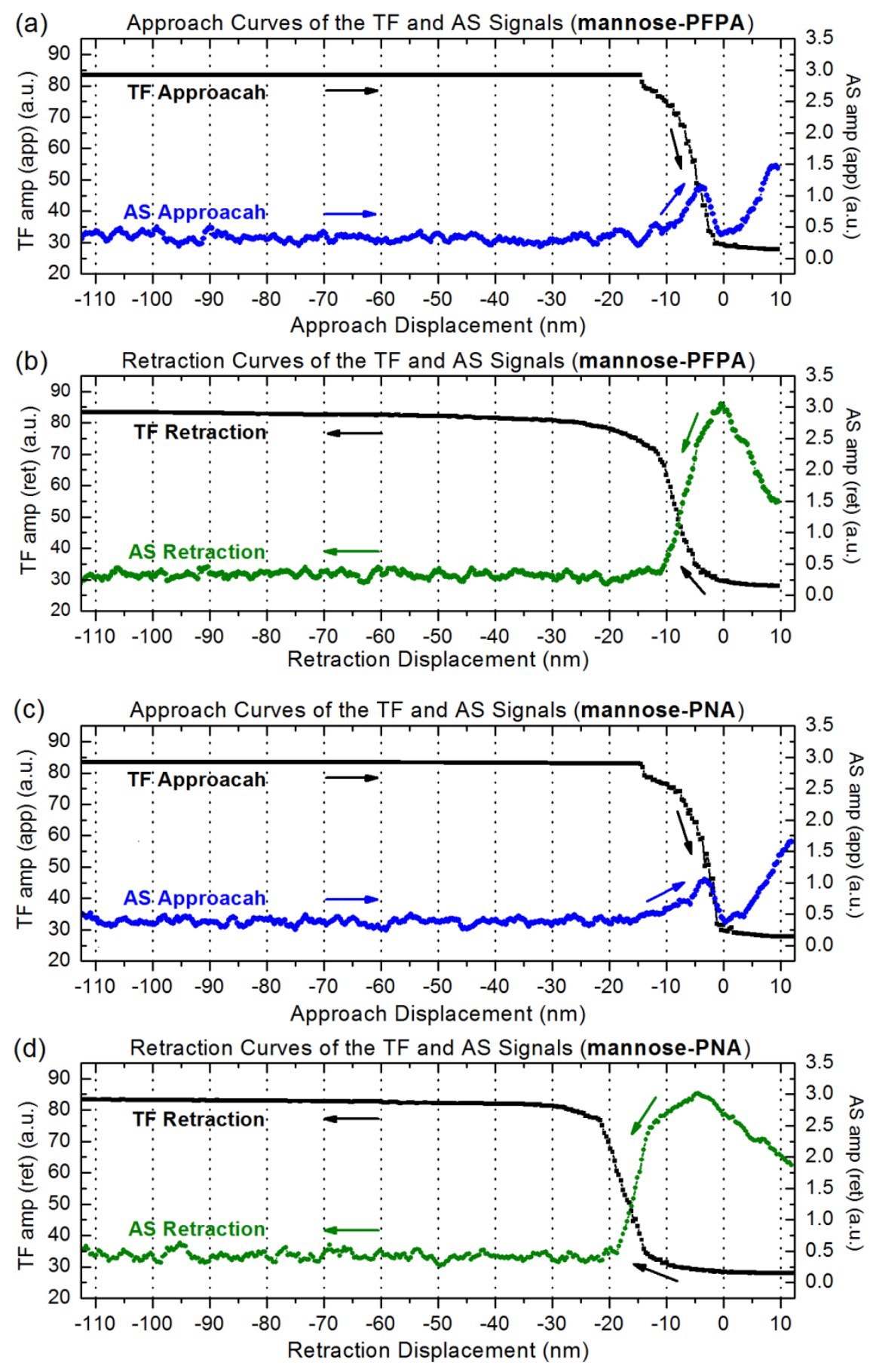

Figure 8.5: Control experiments performed on unspecific binding surfaces: approach (a) and retraction (b) curves of TF and acoustic signals for PFPA-coated surface; and approach (c) and retraction (d) curves for PNA-coated substrate. 


\subsection{Conclusions}

In summary, we demonstrated the sensing capability of the shear-force/acoustic microscope for detection of specific carbohydrate-protein interactions, particularly, the rupture event of mannose-Con A complex. As far as our knowledge goes, this is the first report that the shear-force/acoustic technique was successfully employed to probe biological interactions. Con A molecules were covalently immobilized onto a flat silicon wafer, while mannose was covalently tethered to a tapered gold tip, which was excited to oscillate at its resonance frequency. By integrating an acoustic sensor (located under the sample) into a tuning fork-based shear-force microscope, responses of the carbohydrateterminated probe as well as the protein-attached substrate are expected to be concurrently monitored when binding/unbinding process takes place. Specific peaks above the noise level were clearly present on the retraction curves of both the TF and acoustic measurements, which indicated rupture events of bonds formed between mannose and Con A molecules when the tip was close enough to the surface. The shear-force/acoustic detection platform is able to provide a qualitative impression of detachment of mannose from Con A. The experimental results were consistent to those obtained using the conventional AFM-based force spectroscopy method. Furthermore, control experiments were carried out to confirm the measured unique signals represent bond rupture and to differentiate the specific binding from non-specific bindings. Our studies successfully demonstrated the shear-force/acoustic microscope has great potentials as an alternative and powerful tool to investigate fundamental aspects of biological events. This detection 
framework should also prove valuable in a number of characterization applications and nanofluidic sensor devices development. 


\section{Chapter 9. Electric Field-Assisted Dip-Pen Nanolithography on P4VP Thin Films}

\subsection{Introduction}

Nanolithography comprises a set of technologies capable of etching, writing, or patterning materials with nanometer scale precision, which nowadays has widespread applications in electronic engineering, physics, chemistry and biology. Dip-pen nanolithography (DPN) ${ }^{152}$, as a scanning-probe-based lithography, is used to directly deliver atoms or molecules (initially attached by adhesion to an atomic force microscope (AFM) tip) onto substrates of interest in a controlled fashion. A diverse range of molecular species such as small organic molecules ${ }^{152}$, biological macromolecules ${ }^{153}$, conducting polymers ${ }^{154}$, metal ions and nanopaticles ${ }^{155,156}$, have been deposited via DPN process onto surfaces of proper affinities. In addition, the patterning substrates have been expanded to metals ${ }^{157}$, insulators ${ }^{157}$, semiconducting materials ${ }^{158}$, and functional monolayers adsorbed on substrates ${ }^{159}$. Applications of DPN have been envisioned in nanoelectronics ${ }^{160}$, microfluidics ${ }^{161}$, molecular electronics (organic circuits) ${ }^{162}$, and nanosensors for biological and chemical species ${ }^{163}$, etc. In most cases, the transport of “ink" molecules from the tip to the substrate occurs in a liquid bridge naturally formed between them through capillary condensation. Hence, this technique has also been used to experimentally study the mechanism of molecular diffusion through nanoscale junctions and the in situ growth of self-assembled monolayers under ambient conditions $^{164,165}$. Furthermore, due to its direct-write capability, DPN is suitable for investigating responsive films at the mesoscopic scale. 
Responsive films made of self-assembled monolayers (SAMs) or polymer thin films are valuable in the development of electronic, analytical and biomedical devices. These films exhibit different properties in response to external signals, such as the changes in temperature, $\mathrm{pH}$, light, and ionic strength. Poly(4-vinylpyridine) (P4VP), a weak polyelectrolyte, can be prepared as polymer thin films tethered to solid supports simply by spin coating and subsequent UV irradiation ${ }^{99}$. The immobilization results from the UV induced cross-linking of polymer leading to the attachment of polymer film to the substrate. Moreover, P4VP film shows a pH-dependent response that causes the structural changes of the polymer and controls surface wettability ${ }^{166}$. The reversible transformation of P4VP originates from the protonation of pyridine units of the polymer backbone at the acidic $\mathrm{pH}$, thus providing the basis for applications of $\mathrm{P} 4 \mathrm{VP}$ films as responsive coatings. Recently, P4VP polymer-brush-functionalized electrodes have been designed for future “smart” bioelectronic devices ranging from biosensors to biofuel cells ${ }^{167,168}$. Additionally, P4VP is an interesting material because of its tunable photoluminescence (PL) properties. Vaganova et al. demonstrated that P4VP with protonated polymeric pyridine groups displayed photoactive emission properties, which could be tuned to glow blue, red or green, depending on the pumping wavelength ${ }^{169}$.

Herein we report the fabrication of patterns on P4VP thin films based on DPN technique by exploiting the swelling of the polymer. Differing from the feature formation via a direct deposition of ink molecules onto substrates, the underlying principle in our approach is to locally trigger the protonation of P4VP for creating swollen patterns by delivering acidic ions into the polymer. Moreover, a reliable strategy for well controlling 
the patterning process results when applying an electric field between the AFM tip and the sample. We demonstrate the capability of the electric field-assisted DPN technique for reproducibly and reliably fabricating nanostructures. Our work includes a systematic study of pattern formation under different patterning parameters (i.e., externally applied bias and tip-sample contact force), and more importantly, provides evidence on the reversible characteristic of this process.

\subsection{Experimental Section}

Polymer Thin Film Preparation: P-type silicon (100) wafers were cleaned by immersion in piranha solution $\left(35 \% \mathrm{H}_{2} \mathrm{O}_{2} / \mathrm{H}_{2} \mathrm{SO}_{4}=3: 7(\mathrm{v} / \mathrm{v})\right)$ at $80-90^{\circ} \mathrm{C}$ for $60 \mathrm{~min}$ and were subsequently washed with boiling water for 90 min, then dried under $\mathrm{N}_{2}$. The fleshly cleaned wafers were then dipped into HF aqueous solution to remove the native oxide layer from the silicon surface, rinsed thoroughly with Milli-Q-water, and dried with $\mathrm{N}_{2}$. A $10 \mathrm{mg} / \mathrm{ml}$ solution of P4VP (molecular weight ca. 160000) in n-butanol was spin coated onto silicon substrates at $2000 \mathrm{rpm}$ for $60 \mathrm{~s}$. The polymer-coated substrates were irradiated with a $450 \mathrm{~W}$ medium-pressure mercury lamp for 15 min (the irradiation time included approximate 2 min warm up for the UV lamp to reach its maximal intensity). The unbound polymers were removed by soaking the irradiated substrates in n-butanol for $12 \mathrm{~h}$. The remaining films were dried with $\mathrm{N}_{2}$, and the thickness was measured on a Gaertner Model L116A ellipsometer with a $632.8 \mathrm{~nm} \mathrm{He} / \mathrm{Ne}$ laser at an incident angle of $70^{\circ}$. The refractive index used in the model to determine the film thickness was 1.581 for 
P4VP. An average thickness of $40 \mathrm{~nm}$ was obtained after taking measurements at three different locations.

Force Calibration: Silicon-based AFM tips of high spring constant coated with 15nm thick wear-resistant platinum (NSC15/Pt, $k \approx 40 \mathrm{~N} / \mathrm{m}$, MikroMasch) were used for surface patterning and imaging. The normal spring constant of each cantilever was calibrated using the Sader method ${ }^{170}$, which states $k=0.1906 \rho_{f} b^{2} L Q_{f} \omega_{f}^{2} \Gamma_{i}^{f}\left(\omega_{f}\right)$, where $L$ and $b$ are the length and width of the cantilever, respectively, $\rho_{f}$ is the density of air $\left(1.18 \mathrm{~kg} / \mathrm{m}^{3}\right.$ at $\left.25^{\circ} \mathrm{C}\right), \omega_{f}$ and $Q_{f}$ are the resonant frequency and quality factor of the fundamental resonance peak in air, respectively, and $\Gamma_{i}^{f}$ is the imaginary part of the hydrodynamic function.

Feature Patterning and Characterization: All the experiments were performed with a commercial AFM (XE-70, Park Systems) in ambient environment $\left(23-24^{\circ} \mathrm{C}\right.$ and $45-55 \%$ relative humidity). Prior to the patterning process, the AFM tip was inked by dipping into a phosphate buffer solution for $1 \mathrm{~min}$ and blown dry with $\mathrm{N}_{2}$. Buffer solutions (100 mM) of $\mathrm{pH} 4.0$ and $\mathrm{pH} 8.3$ were prepared by dissolving $\mathrm{NaH}_{2} \mathrm{PO}_{4}$ and $\mathrm{Na}_{2} \mathrm{HPO}_{4}$ in appropriate proportion in distilled water. The sample was grounded while a bias was applied to the tip. The voltages were quoted by referencing the polarity of the tip. The bias voltage was held constant while the tip transcribed the preprogrammed patterns controlled by the AFM in contact mode. To visualize the resulting patterns, topographic images were subsequently acquired by operating the AFM in non-contact mode with the same tip used for patterning. 


\subsection{Results and Discussions}

As outlined in Figure 9.1, a series of systematic experiments were carried out to study the mechanism of pattern formation as well as the effects of patterning parameters (e.g., externally applied bias and tip-sample contact force). To explore the role of externally applied bias in the patterning process, a number of bias voltages ranging from $-5 \mathrm{~V}$ to $5 \mathrm{~V}$ were applied. The AFM tip was immersed into the acidic phosphate buffer solution of $\mathrm{pH}$ 4.0. During the patterning operation, the inked tip was scanned across the polymer surface at a writing speed of $80 \mathrm{~nm} / \mathrm{s}$ while the contact force (e.g., $1 \mu \mathrm{N}$ ) between the tip and surface was held constant. When no bias voltage was applied (Figure 9.1a), the pattern formation was neither noticeable nor reproducible. When the tip was negatively biased (Figure 9.1b), no structure was present. By contrast, swollen patterns consisting of continuous lines were obtained when the tip was positively biased (Figure 9.1c).

Topographic images in Figure 9.2 show that the heights of the selected features are $13 \mathrm{~nm}$ (Figure 9.2a), $6 \mathrm{~nm}$ (Figure 9.2b) and $3 \mathrm{~nm}$ (Figure 9.2c), corresponding to the applied bias voltages of $5 \mathrm{~V}, 3 \mathrm{~V}$ and $0 \mathrm{~V}$, respectively. To test the influence of tip-substrate contact force on the pattern formation, experiments similar to the one described above were repeated using a fixed bias voltage (e.g., $5 \mathrm{~V}$ ) and varied contact forces ranging from $0.5 \mu \mathrm{N}$ to $1 \mu \mathrm{N}$. As shown in Figure 9.2, the heights of the selected features are 8 $\mathrm{nm}$ (Figure 9.2d) and $3 \mathrm{~nm}$ (Figure 9.2e), corresponding to the contact forces of $0.8 \mu \mathrm{N}$ and $0.5 \mu \mathrm{N}$, respectively. When the patterning operation was attempted using an uncoated tip (Figure 9.1d), swollen pattern could not be obtained even if the tip was positively biased. 

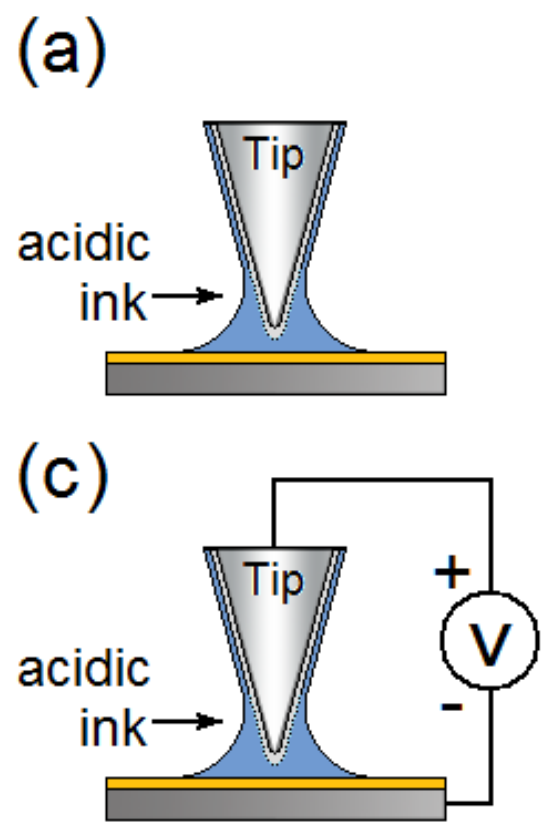
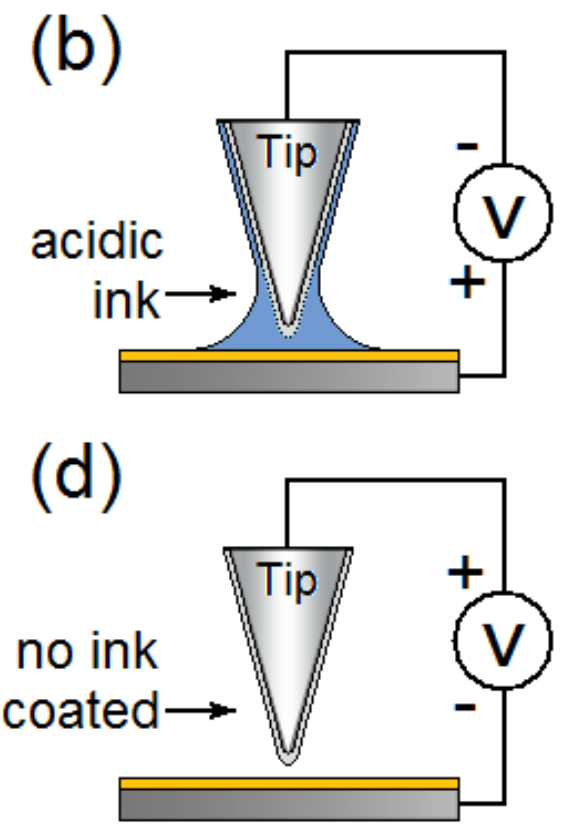

Figure 9.1: Different experimental settings used for patterning features on P4VP polymer films: A tip was inked with the acidic buffer and (a) not biased, (b) negatively biased, (c) positively biased. (d) An uncoated tip while applying a positive bias.

This technique was also able to produce features with sub- $100 \mathrm{~nm}$ width and complex patterns. A line feature with width down to $60 \mathrm{~nm}$ was created (Figure 9.2f), along with a pattern of the letters: PSU (Figure 9.2g), in a $5 \mu \mathrm{m} \times 5 \mu \mathrm{m}$ square region. In the latter, the average line width is about $180 \mathrm{~nm}$. 

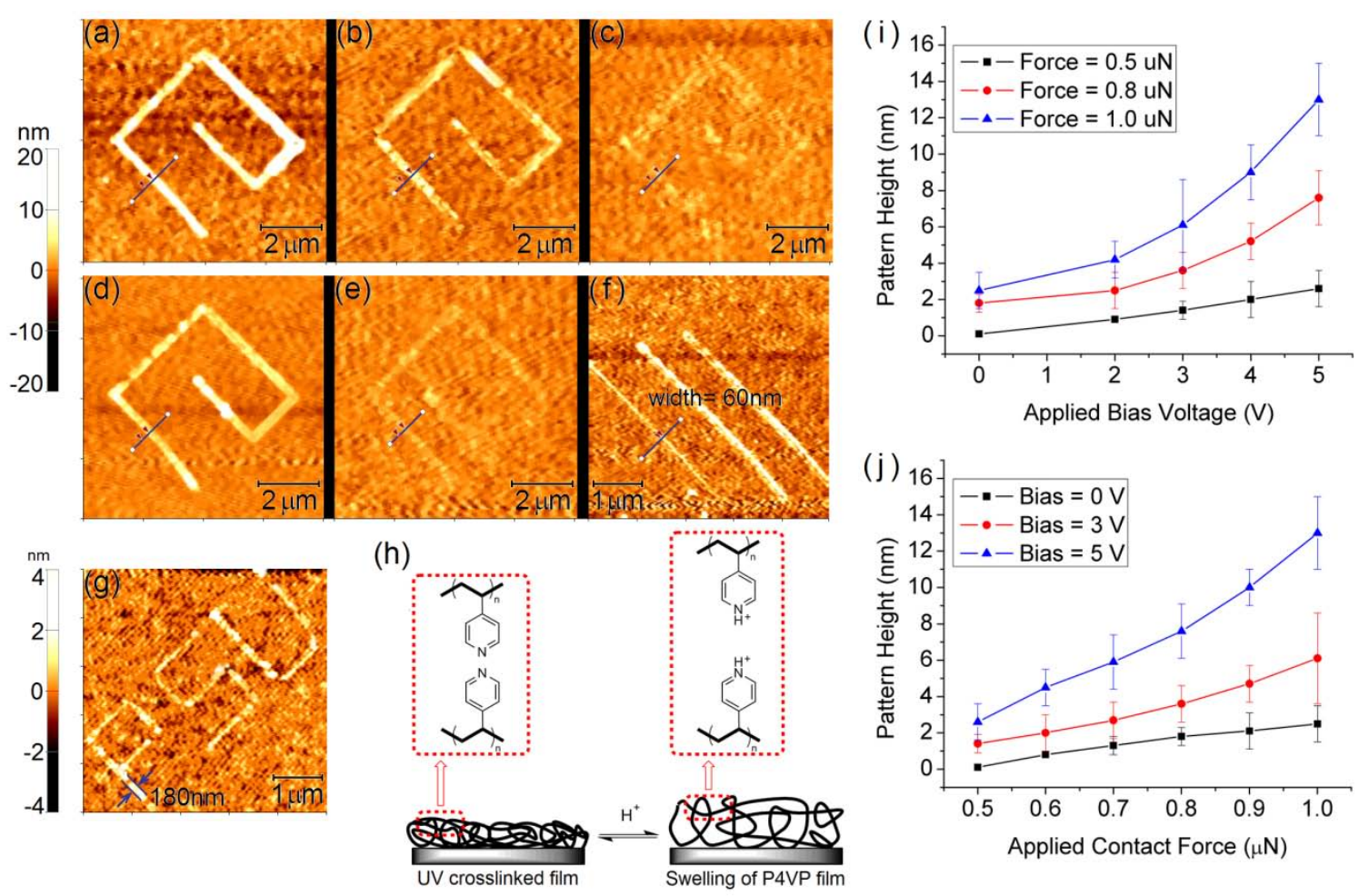

Figure 9.2: (a-e) Topographic images of features drawn using the tip coated with acidic ink. The applied bias voltages $(\mathrm{V})$, contact forces $(\mu \mathrm{N})$ and measured heights of selected lines (nm) are the following: (a) 5, 1, 13; (b) 3, 1, 6; (c) 0, 1, 3; (d) 5, 0.8, 8; (e) 5, 0.5, 3. (f) A line feature with $60 \mathrm{~nm}$ wide. (g) A pattern of the letters: PSU. Image sizes: $8 \mu \mathrm{m} \times 8$ $\mu \mathrm{m}$ for (a-e); $5 \mu \mathrm{m} \times 5 \mu \mathrm{m}$ for (f-g). (h) Schematic showing that the swelling of P4VP film originates from the protonation of the pyridine units. (i) The pattern height (nm) v.s. bias voltages (V) at various fixed contact forces. (j) The pattern height (nm) v.s. contact forces under different constant bias voltages.

To further support the claim that the presence of raised patterns was resulted from the swelling response of the P4VP polymer film ${ }^{166,171}$, reversibility of the patterning process was demonstrated by selectively erasing an existing swollen pattern. "Dot” features were initially generated using a tip coated with acidic ink. Only the dot at the center of Figure 9.3 (denoted by the dashed-line square) was then twice subjected to the "erasing" operation, which consisted of using the same tip but inked with a basic phosphate buffer solution of $\mathrm{pH} 8.3$ without applying the bias voltage. The removal of the existing dot was 
performed by keeping the tip in contact and stationary on top of the dot (no scanning movement). The images in Figure 9.3 show the progressive attenuation of the dot height, from $12 \mathrm{~nm}$ to $7 \mathrm{~nm}$ and finally to $3 \mathrm{~nm}$. During the "erasing” process, the applied load exerted by the tip was kept intentionally low (i.e., $0.5 \mu \mathrm{N}$ ) so as to minimize potential damages to the dot pattern. Indeed, there was no change in the feature height when the “erasing” experiment was attempted using an uncoated tip and $0.5 \mu \mathrm{N}$ load. Altogether, this result indicates that the disappearance of the central dot in Figure 9.3 was not due to mechanical damages caused by the tip but the presence of basic buffer solution. 


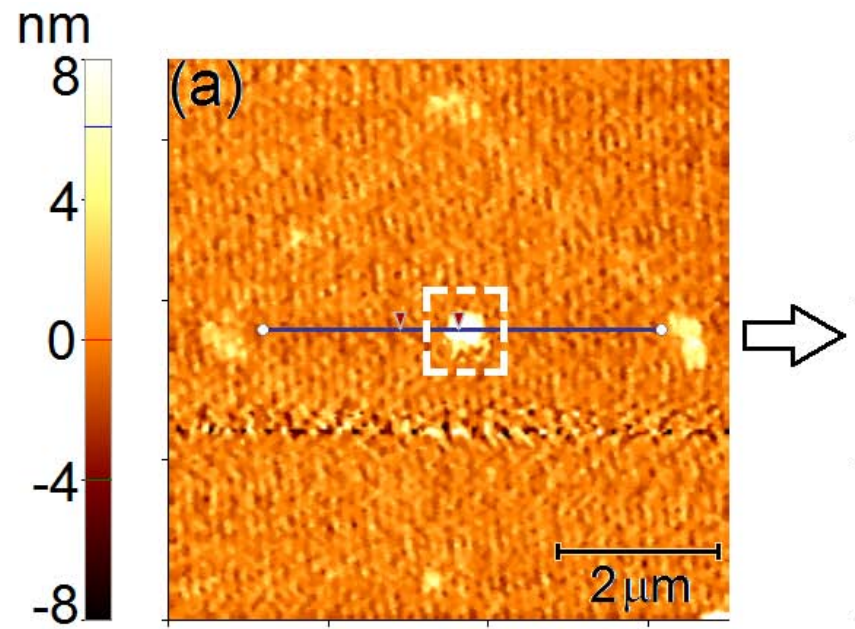

(d)

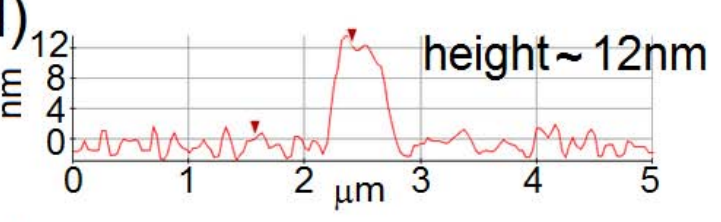

(e)

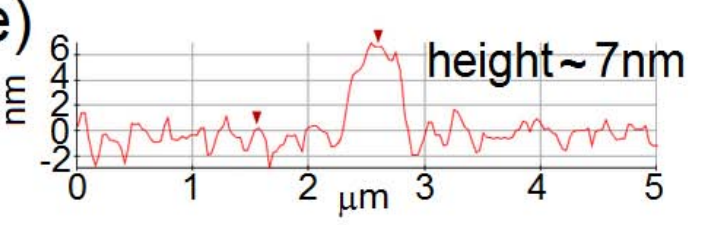

(f)
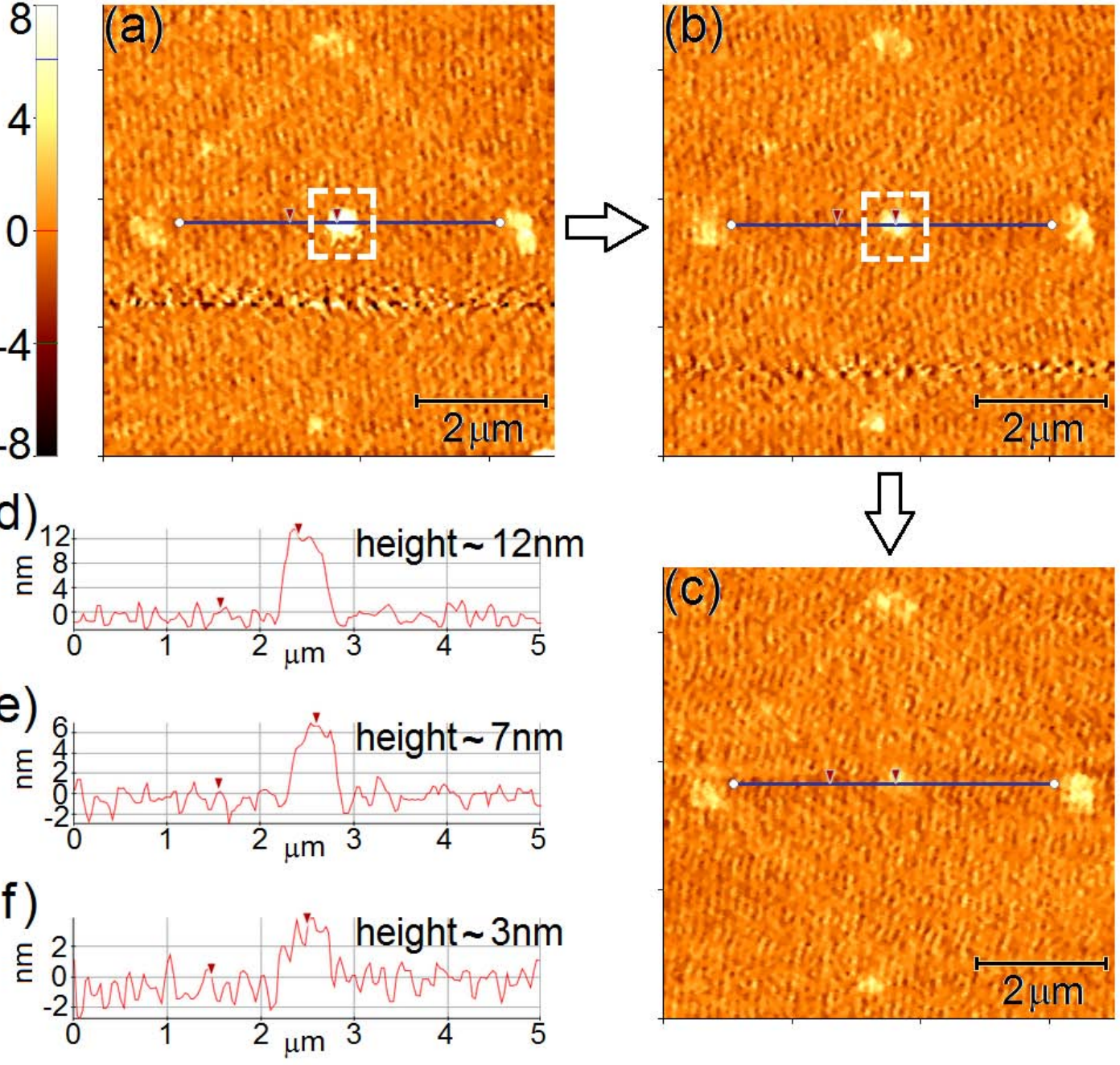

Figure 9.3: Topographic images show the removal of the dot pattern at the center: (a) Initially, the dot height was $12 \mathrm{~nm}$; (b) After the first erasing performance, the height decreased to $7 \mathrm{~nm}$; (c) After the second erasing performance, the remaining height was only $3 \mathrm{~nm}$. Line profiles of (d), (e), and (f) correspond to the features selected in (a), (b), and (c), respectively.

Experimental results presented herein suggest that the essence of pattern formation on P4VP film is the local protonation of pyridine units of the polymer with hydronium ions $\left(\mathrm{H}_{3} \mathrm{O}^{+}\right)$. As outlined in Figure 9.2h, the abundant acidic ions (i.e., $\left.\mathrm{H}_{3} \mathrm{O}^{+}\right)$in the acidic ink delivered into the film cause the protonation of pyridine groups, yielding the pyridinium groups whose Coulomb repulsions between the charged centers make the polymer swell. 
Moreover, the attenuation of existing feature's height with subsequent deposition of the basic ink reveals that the swollen patterns are generated by polymer protonation rather than residual salt accumulation from the buffer solution. This observation provides the evidence on the reversible characteristic of the patterning process as well.

A conventional DPN process involves two steps: (1) the ink molecules transport from the coated tip to the substrate though the water meniscus that naturally forms between them, and (2) ink is adsorbed onto the surface to form a uniform monolayer. In our experiments, two major factors in controlling the properties of scribed patterns were found: the externally applied bias and tip-sample contact force. Comparison among the patterns in Figure 9.2 indicates that higher bias voltages applied between the tip and sample yield lines with increasing heights at a fixed contact force $(1 \mu \mathrm{N})$. The influence of applied bias on feature height was further verified at different contact forces. Plots shown in Figure 9.2i present the same trend in the height increase along with ascending the external voltage. It is well-known that the electric field across the polymer film is correlated to the bias voltage. A significant increase in the pattern's height is observed as the applied field strength is increased, indicating that the efficiency of acidic ion transport is electric-field dependent. Owing to the presence of applied electric field, the electrostatic force acts as a driving force to facilitate the deposition of acidic ions. Furthermore, since the patterning experiments were carried out in ambient conditions and the tip was initially dried with nitrogen, the electric field was required to anchor acidic ions to the substrate, so that the diffusive transport could occur consistently. In addition, the influence of Joule-heating discussed in electrostatic nanolithography ${ }^{172,173}$ was taken 
into consideration. In the experiment presented above, no swollen pattern was obtained by using an uncoated tip when the tip was positively biased. This result indicates that the pattern formation cannot be attributed to the Joule heating alone. However, since the ion mobility is greatly enhanced when the temperature increased ${ }^{174}$, we cannot completely eliminate the potential effect of Joule-heating. It could also be considered as a way to facilitate the ion transport.

Although the electric field has been utilized in electrochemical dip-pen nanolithography (E-DPN) ${ }^{175}$, the role of electric field played in our approach differs from in E-DPN where metallic or semiconducting structures are fabricated via electrochemical reactions. By contrast, no electrochemical reaction is needed for fabricating patterns on P4VP film, the external electric field applied during the patterning process simply made the surface patterning more reliable and repeatable.

Similar dependence of pattern height on contact force was found in our experiments: higher features arose from stronger contact force, which contrasted to the no effect on contact force reported by Weeks et al. ${ }^{176}$. The dependencies of pattern height on contact forces under different constant bias voltages are summarized in Figure 9.2j. The increase in the contact force exerted on the tip could lead to a greater tip area in contact with the polymer. Alternatively, this could result in broadening of the liquid meniscus between the tip and surface. Therefore, the increase in pattern's height with increasing the contact force could be attributed to the wider tip-sample effective contact area, where a greater amount of acidic ions could be transported from the tip to the polymer. 
The breakdown of polymer film did not take place under the applied electric fields (bias voltages are from $-5 \mathrm{~V}$ to $5 \mathrm{~V}$ ). Additionally, no effect of external bias voltage on the vertical position of the tip was observed by monitoring the deflection of the AFM cantilever. It reveals that the electric force induced by the bias voltage can be eliminated. The high spring constant of non-contact AFM cantilevers (compared to general contact AFM cantilevers) was of significance for precise control of contact force during the patterning process.

The factors that affect the dimensions of the patterned features can be attributed but not limited to a combination of several variables, such as the writing speed ${ }^{159,176,177}$, the effects of temperature and humidity ${ }^{164,176,178}$, and the geometry and wettability of the $\operatorname{tip}^{178}$. The dimensions of dot features as a function of the dwell time and relative humidity has been demonstrated in our previous work ${ }^{171}$. No structures could be created when the humidity was below $30 \%$. It indicates that the transfer of acidic ions takes place when sufficient liquid is present between the tip and the sample. Furthermore, using a sharp tip on which remaining liquids were removed just before the patterning process was critical in achieving finer features.

\subsection{Conclusions}

In conclusion, we demonstrated the fabrication of nanostructures on cross-linked P4VP thin films based on DPN technique. The protonation of the pyridine units of P4VP responsive to the acidic ions delivered into the film leads to the swollen pattern formation. Furthermore, we reported that the application of an electric field between the tip and the 
sample provides a simple method to precisely control the patterning process. The acidic ions (i.e., $\mathrm{H}_{3} \mathrm{O}^{+}$) are reliably transferred from the ink-coated tip to the polymer-covered substrate under the applied electric field. The effects of patterning parameters, such as applied bias and contact force, were investigated. The results suggest that both a higher bias voltage and a stronger contact force result in a marked increase in the pattern's height. Significantly, we illustrated that the removal of existing structures on P4VP films can be achieved by taking advantage of the reversible property of pattern formation. 


\section{Chapter 10. Final Conclusions}

Shear-force mechanism nowadays has been widely used in scanning probe microscopes (SPMs) for probe-sample distance regulation. Briefly speaking, it refers to the probe-sample interaction that causes a net decrease in the amplitude of the SPM probe's oscillation when the probe is brought in proximity to the sample surface (within $\sim 10 \mathrm{~nm}$ ). The typically monotonic dependence of the probe's oscillation amplitude and the probe-sample distance allows implementing a feedback position control regulation. In practice the shear-force effect is sufficient for obtaining high quality images of relatively large (micron-sized) standard samples of uniform composition. However, its origin is still not be well explained by present theoretical and experimental studies, which causes uncertainty in imaging interpretation when analyzing samples of heterogeneous composition, particularly at the nanoscale. The shear-force mechanism for distance regulation exploits the fact that under ambient conditions, moisture and other hydrocarbon molecules in the air are attracted to material surfaces, leading to a mesoscopic fluid-like layer of a few-nanometer thickness. Our goal in this dissertation was to gain an insight into the key of interactions between the probe and the sample in mesoscopic fluid films using the shear-force/acoustic near-field microscope, which is a new system being developed by our research group. At the core of this development is the integration of acoustic sensors into a tuning fork-based shear-force microscope. To achieve shear force detection with high lateral resolution, a sharpened gold tip is used as a probe, which is manually mounted to one prong of a quartz tuning fork (TF) of $32 \mathrm{kHz}$ 
nominal resonance frequency. Gold tips can be reproducibly obtained through electrochemical etching. The sample substrate can be either a silicon wafer or an atomically flat mica disk. Chemical treatments (i.e., cleaning and chemical modification) are performed prior to the use in order to meet the requirements of specificity and reproducibility of the experimental trials. In general, the TF is electrically driven to vibrate at its resonance frequency in such a way that the tip oscillates parallel to the sample surface. Measurement of the electrical signal generated by the TF is based on the piezoelectric effect of quartz crystals. Aside from the TF signal in response to variations in the probe's amplitude, we also place an acoustic sensor under the substrate. It is used to detect the acoustic waves produced while the probe end oscillates in the mesoscopic fluid layer. The demonstrated sensitivity of this acoustic technique serves as an extra analytical probe that can potentially contribute to the characterization of the shear-force effect. Our dual sensing (shear-force and acoustic) system allows concurrent detection of shear forces exerted on the tip and acoustic waves generated due to the vibrating tip in the liquid. Information is expected to be extracted from the approach/retraction curves which plot the TF electric current and the generated acoustic signal as a function of the probesample distance, providing both shear-force and acoustic interpretations of the probesample interaction.

In ambient atmosphere the probe vibrates with steady amplitude, generating sound waves received by the bottom acoustic sensor as a nonzero background. Typically, as the probe approaches to the sample, it comes in contact with the mesoscopic fluid layer, which causes a decrease in its vibration amplitude. However, a corresponding change in 
the acoustic signal is usually not detectable because it is not above the acoustic transducer noise level. As the tip gets more immersion into the mesoscopic film, the TF signal further decreases due to the viscoelastic interaction with the fluid. Simultaneously, the acoustic signal increases due to the distance-dependent phase lagging between the vibrating tip and the fluid layer which becomes more pronounced with the ongoing approach process. If the probe is forced to penetrate deeper in the liquid film, mechanical contact of the tip with the surface, like an intermittent knocking of the tip on the surface, will take place; this situation leads to decreases in both the TF and acoustic signals. In this dissertation, we carried out a series of systematic experiments to investigate factors that have impacts on probe-sample interactions. We discussed effects of tip geometry, relative humidity of the environment, the nature of sample surfaces (including cleanliness, stiffness, and hydrophilicity/hydrophobicity), and velocity of the tip’s movement on approach/retraction measurements. Varying these parameters gives rise to curves with different shapes that reflect changes in properties of the mesoscopic fluid layer. For example, the tip with large radius of apex (chubby tip) is influenced more by the mesoscopic fluid layer than that having smaller radius of apex (sharp tip). An increase in environmental humidity is expected to lead to a growth of the contamination layer. By performing cleaning of samples before their usage, hysteresis from the mesoscopic film is much reduced (even avoid), providing excellent reference samples. The latter can be post purposely modified and thus determine the corresponding effects. The extent of the maximum-to-minimum transition region on the TF and acoustic retraction curve are found to depend on the speed at which the measurements are performed. More detailed 
discussion was given in the corresponding chapter. At the end of that chapter, we reported an observation that the acoustic signal exhibited contrast when placing the tip above surfaces covered by different materials (i.e., a glass cover slip with a gold coating only on one half of the substrate). The result reveals potential application of using the acoustic technique to image samples composed of different materials.

As we mentioned above, the interaction between the probe and the sample (including the mesoscopic fluid layer) depends on the probe-sample distance. Therefore, an estimation of the actual position of the tip relative to the surface is helpful in exploring the origin of interactions at different distances. For metal tips and conductive surfaces (one of them connected to a voltage bias), monitoring the eventual current signal is a feasible method to indicate the onset of the tip apex in mechanical contact with the sample surface. We implemented detection of contact current into our shearforce/acoustic microscope, and therefore multiple probing techniques were concurrently utilized in the study of the probe-sample interaction by exciting acoustic waves in nearfield fashion. A compelling finding was the acoustic signal was detected before contact current appeared. It suggests that the acoustic waves can be generated without mechanical contact of the tip end with the hard surface. Owing to the viscoelastic properties of the mesoscopic fluid layer, the amplitude of the vibrating tip monotonically decreases as a result of a combination of damping of its amplitude and blue-shift of its resonance frequency. The measured acoustic signal is either negatively or positively correlated to the TF signal. Detection of contact current helps elucidate the relationship between these two signals: they are negatively correlated while there is a lack of current 
signal, which reveals the acoustic signal rises because of an increasing phase lagging between the tip apex and the fluid layer at shorter probe-sample distance; they are positively correlated upon an observation of current pulses, which reveals that an intermittent contact between the tip and the surface contributes to decreases in both the TF and acoustic signals. Additionally, the onset of current signal is affected by properties of the tip and the sample. For example, by purposely tilting the tip axis relative to the normal to the surface the probe-sample current appears earlier.

To gain understanding of the generation of acoustic waves, we performed analogous experiment in bulk liquid using the same shear-force/acoustic technique. When a cylindrical optical fiber laterally vibrates in a liquid droplet, the liquid exerts a viscous effect to its dynamic behavior. Herein, the fiber's mechanical oscillation is coupled to the liquid to produce sound waves that propagate through the droplet bulk and couple to the substrate. Notice this is quite similar to our mesoscopic-scale experiments. As the immersion depth increases, a remarkable red-shift of resonance frequencies was observed in measurements of both TF and acoustic signals. The red-shift is attributed to the increase in mass loading applied to the fiber when it penetrates deeper into the liquid (it differs from the blue-shift of the probe's resonance frequency when the tip is immersed in the mesoscopic fluid layer). Furthermore, with the decrease in the amplitude of the probe's vibration in liquid, the amplitude of acoustic signal increases. Glycerol-water solutions with different viscosities and fibers with various diameters were used in the experiment. Our results show that the generation of acoustic signal is related to the properties of the probe (fiber) and the medium (liquid). At a constant immersion depth, 
either dipping the probe into more viscous liquid or using the fiber with larger diameter helps produce larger acoustic signal.

The fluid-like thin film on solid surfaces plays a role as a natural environment in carbohydrate-protein binding studies. We also demonstrated the sensing capability of our shear-force/acoustics technique to probe biological interactions, particularly, rupture events of chemical bonds between mannose and Concanavalin A (Con A) molecules. Con A molecules were covalently immobilized onto a flat silicon wafer; while mannose was covalently tethered to a tapered gold tip, which was excited to oscillate at its resonance frequency. By bringing the tip close to or away from the surface, approach/retraction curves of TF and acoustic signals were concurrently recorded as a function of the probe's displacement. We expect to receive specific signals indicative of bond formation or/and breakage on the resulting curves. Indeed, peaks and valleys above the noise level are clearly visible on the retraction curves of both the TF and acoustic measurements. They can be thought to be an indication of the rupture of mannose-Con A pairs. When the tip is brought close enough to the sample, hydrogen bonds form and lead to a strong coupling between them. The enhanced coupling is able to efficiently amplify the acoustic signal produced by the tip's mechanical motion. In addition, the protein molecules being dragged to laterally move by the vibrating tip also has contribution to generate acoustic waves detected by the bottom sensor. As the tip further retracts from the sample, the rupture occurs and the mannose-terminated tip is released from the Con A coated surface. Correspondingly, we detect specific signals on the TF and acoustic retraction curves. To confirm the measured unique signals represent rupture of specific binding between 
mannose and Con A molecules, control studies were performed by repeating the same experiment using the same mannose-coated tip on unspecific binding surfaces. None of specific peaks were found on these curves.

Finally, the existence of liquid layers is utilized to create nanostructures via dip-pen nanolithography (DPN). After dipping an AFM cantilever into an acid solution, hydronium ions can be delivered from the AFM tip into a poly(4-vinylpyridine) (P4VP) thin film through the naturally formed liquid bridge between them, leading to local swelling of the polymer. Our method for fabricating swollen patterns is based on the protonation mechanism, which differs from the conventional DPN where the patterned features are formed via a direct deposition of ink molecules onto substrates. To improve reproducibility and reliability, we applied an electric field between the AFM tip and the sample during the patterning procedure. The dimension of generated features was related to the applied bias voltage and the probe-sample contact force. We also demonstrated that this process is reversible by deswelling the existing patterns using a base solution. 


\section{References}

(1) Binnig, G.; Rohrer, H.; Gerber, C.; Weibel, E. Physical Review Letters 1982, 49, 5761.

(2) Binnig, G.; Quate, C. F.; Gerber, C. Physical Review Letters 1986, 56, 930-933.

(3) Colton, R. J.; Baselt, D. R.; Dufrêne, Y. F.; Green, J.-B. D.; Lee, G. U. Current Opinion in Chemical Biology 1997, 1, 370-377.

(4) Betzig, E.; Trautman, J. K.; Harris, T. D.; Weiner, J. S.; Kostelak, R. L. Science 1991, 251, 1468-1470.

(5) Binnig, G.; Rohrer, H.; Gerber, C.; Weibel, E. Applied Physics Letters 1982, 40, 178180.

(6) Bryant, P. J.; Kim, H. S.; Zheng, Y. C.; Yang, R. Review of Scientific Instruments 1987, 58, 1115.

(7) Fink, H.-W. Physica Scripta 1988, 28, 260.

(8) Ibe, J. P.; P. P. Bey, J.; Brandow, S. L.; Brizzolara, R. A.; Burnham, N. A.; DiLella, D. P.; Lee, K. P.; Marrian, C. R. K.; Colton, R. J. Journal of Vacuum Science \& Technology

A: Vacuum, Surfaces, and Films 1990, 8, 3570-3575.

(9) Bryant, A.; Smith, D. P. E.; Quate, C. F. Applied Physics Letters 1986, 48, 832-834.

(10) García, R.; San Paulo, A. Physical Review B 1999, 60, 4961-4967.

(11) Giessibl, F. J. Science 1995, 267, 68-71.

(12) Hansma, P. K.; Cleveland, J. P.; Radmacher, M.; Walters, D. A.; Hillner, P. E.;

Bezanilla, M.; Fritz, M.; Vie, D.; Hansma, H. G.; Prater, C. B.; Massie, J.; Fukunaga, L.;

Gurley, J.; Elings, V. Applied Physics Letters 1994, 64, 1738-1740.

(13) Kirk, M. D.; Albrecht, T. R.; Quate, C. F. Review of Scientific Instruments 1988, 59, 833-835.

(14) Kim, Y.; Lieber, C. M. Science 1992, 257, 375-377.

(15) Piner, R. D.; Zhu, J.; Xu, F.; Hong, S.; Mirkin, C. A. Science 1999, 283, 661-663.

(16) Betzig, E.; Trautman, J. K. Science 1992, 257, 189-195.

(17) Pohl, D. W.; Denk, W.; Lanz, M. Applied Physics Letters 1984, 44, 651-653.

(18) Harootunian, A.; Betzig, E.; Isaacson, M.; Lewis, A. Applied Physics Letters 1986, 49, 674-676.

(19) Betzig, E.; Isaacson, M.; Lewis, A. Applied Physics Letters 1987, 51, 2088-2090.

(20) Buratto, S. K.; Hsu, J. W. P.; Trautman, J. K.; Betzig, E.; Bylsma, R. B.; Bahr, C. C.; Cardillo, M. J. Journal of Applied Physics 1994, 76, 7720-7725.

(21) Fischer, U. C. Journal of Vacuum Science \& Technology B: Microelectronics and Nanometer Structures 1985, 3, 386-390.

(22) Fischer, U. C.; Durig, U. T.; Pohl, D. W. Applied Physics Letters 1988, 52, 249-251.

(23) Cline, J. A.; Barshatzky, H.; Isaacson, M. Ultramicroscopy 1991, 38, 299-304.

(24) Novotny, L.; Sánchez, E. J.; Sunney Xie, X. Ultramicroscopy 1998, 71, 21-29.

(25) Sánchez, E. J.; Novotny, L.; Xie, X. S. Physical Review Letters 1999, 82, 4014-4017.

(26) Pettinger, B.; Schambach, P.; Villagómez, C. J.; Scott, N. Annual Review of Physical Chemistry 2012, 63, 379-399.

(27) Betzig, E.; Finn, P. L.; Weiner, J. S. Applied Physics Letters 1992, 60, 2484-2486.

(28) La Rosa, A. H.; Yakobson, B. I.; Hallen, H. D. MRS Proceedings 1995, 406, 189. 
(29) Betzig, E.; Chichester, R. J. Science 1993, 262, 1422-1425.

(30) Trautman, J. K.; Macklin, J. J.; Brus, L. E.; Betzig, E. Nature 1994, 369, 40-42.

(31) Blatchford, J. W.; Gustafson, T. L.; Epstein, A. J.; Vanden Bout, D. A.; Kerimo, J.; Higgins, D. A.; Barbara, P. F.; Fu, D. K.; Swager, T. M.; MacDiarmid, A. G. Physical Review B 1996, 54, R3683-R3686.

(32) Hwang, J.; Tamm, L. K.; Böhm, C.; Ramalingam, T. S.; Betzig, E.; Edidin, M. Science 1995, 270, 610-614.

(33) Garcia-Parajo, M. F.; Veerman, J. A.; Ruiter, A. G. T.; van Hulst, N. F.

Ultramicroscopy 1998, 71, 311-319.

(34) Enderle, T.; Ha, T.; Chemla, D. S.; Weiss, S. Ultramicroscopy 1998, 71, 303-309.

(35) Karrai, K.; Grober, R. D. Applied Physics Letters 1995, 66, 1842-1844.

(36) Karrai, K.; Tiemann, I. Physical Review B 2000, 62, 13174-13181.

(37) Günther, P.; Fischer, U. C.; Dransfeld, K. Applied Physics B: Lasers and Optics 1989, 48, 89-92.

(38) Rensen, W. H. J.; Hulst, N. F. v.; Kammer, S. B. Applied Physics Letters 2000, 77, 1557-1559.

(39) Rychen, J.; Ihn, T.; Studerus, P.; Herrmann, A.; Ensslin, K. Review of Scientific Instruments 1999, 70, 2765-2768.

(40) Lemons, R. A.; Quate, C. F. Applied Physics Letters 1974, 24, 163-165.

(41) Takata, K.; Hasegawa, T.; Hosaka, S.; Hosoki, S.; Komoda, T. Applied Physics Letters 1989, 55, 1718-1720.

(42) Myers, D. In Surfaces, Interfaces, and Colloids: principles and applications; WileyVCH New York, 1999, p 183-186.

(43) Stolen, S.; Grande, T. In Chemical Thermodynamics of Materials: Macroscopic and Microscopic Aspects; Wiley-VCH New York, 2004, p 163-164.

(44) Myers, D. In Surfaces, Interfaces, and Colloids: principles and applications; WileyVCH New York, 1999, p 144-145.

(45) Adamson, A. W.; Gast, A. P. In Physical Chemistry of Surfaces; Wiley-VCH New York, 1997, p 4-6.

(46) Israelachvili, J. N. In Intermolecular and Surface Forces; Academic Press: London, 1991, p 314-316.

(47) Israelachvili, J. N. In Intermolecular and Surface Forces; Academic Press: London, 1991, p 312-314.

(48) Myers, D. In Surfaces, Interfaces, and Colloids: principles and applications; WileyVCH New York, 1999, p 477-478.

(49) Hertz, H. In Journal für die reine und angewandte Mathematik (Crelle's Journal) 1882; Vol. 1882, p 156.

(50) Johnson, K. L.; Kendall, K.; Roberts, A. D. Proceedings of the Royal Society of London. A. Mathematical and Physical Sciences 1971, 324, 301-313.

(51) Derjaguin, B. V.; Muller, V. M.; Toporov, Y. P. Journal of Colloid and Interface Science 1975, 53, 314-326.

(52) Muller, V. M.; Yushchenko, V. S.; Derjaguin, B. V. Journal of Colloid and Interface Science 1983, 92, 92-101.

(53) Maugis, D. Journal of Colloid and Interface Science 1992, 150, 243-269. 
(54) Israelachvili, J. N. In Intermolecular and Surface Forces; Academic Press: London, 1991, p 330-334.

(55) Adamson, A. W.; Gast, A. P. In Physical Chemistry of Surfaces; Wiley-VCH New York, 1997, p 352-355.

(56) Myers, D. In Surfaces, Interfaces, and Colloids: principles and applications; WileyVCH New York, 1999, p 419-420.

(57) Israelachvili, J.; Pashley, R. Nature 1982, 300, 341-342.

(58) Giessibl, F. J. Applied Physics Letters 1998, 73, 3956-3958.

(59) Edwards, H.; Taylor, L.; Duncan, W.; Melmed, A. J. Journal of Applied Physics 1997, 82, 980-984.

(60) Grober, R. D.; Acimovic, J.; Schuck, J.; Hessman, D.; Kindlemann, P. J.; Hespanha, J.; Morse, A. S.; Karrai, K.; Tiemann, I.; Manus, S. Review of Scientific Instruments 2000, 71, 2776-2780.

(61) Dye, D. W. Proceedings of the Physical Society of London 1925, 38, 399.

(62) Rychen, J.; Ihn, T.; Studerus, P.; Herrmann, A.; Ensslin, K.; Hug, H. J.; Schendel, P. J. A. v.; Guntherodt, H. J. Review of Scientific Instruments 2000, 71, 1695-1697.

(63) Cui, X.; Rosa, A. L. Applied Physics Letters 2005, 87, 231907.

(64) Lee, M.; Jahng, J.; Kim, K.; Jhe, W. Applied Physics Letters 2007, 91, 023117.

(65) Ren, B.; Picardi, G.; Pettinger, B. Review of Scientific Instruments 2004, 75, 837841.

(66) Stockle, R.; Fokas, C.; Deckert, V.; Zenobi, R.; Sick, B.; Hecht, B.; Wild, U. P. Applied Physics Letters 1999, 75, 160-162.

(67) Ohtsu, M. In Near-field Nano/Atom Optics and Technology; Springer: Tokyo, 1998, p 38-40.

(68) Toledo-Crow, R.; Yang, P. C.; Chen, Y.; Vaez-Iravani, M. Applied Physics Letters 1992, 60, 2957-2959.

(69) Gregor, M. J.; Blome, P. G.; Schofer, J.; Ulbrich, R. G. Applied Physics Letters 1996, 68, 307-309.

(70) He, G.; Müser, M. H.; Robbins, M. O. Science 1999, 284, 1650-1652.

(71) Froehlich, F. F.; Milster, T. D. Applied Physics Letters 1997, 70, 1500-1502.

(72) Davy, S.; Spajer, M.; Courjon, D. Applied Physics Letters 1998, 73, 2594-2596.

(73) La Rosa, A.; Cui, X.; McCollum, J.; Li, N.; Nordstrom, R. Review of Scientific Instruments 2005, 76, 093707.

(74) Brunner, R.; Marti, O.; Hollricher, O. Journal of Applied Physics 1999, 86, 71007106.

(75) Jang, J.; Schatz, G. C.; Ratner, M. A. The Journal of Chemical Physics 2004, 120, 1157-1160.

(76) Wei, P. K.; Fann, W. S. Journal of Applied Physics 2000, 87, 2561-2564.

(77) Donovan, R. P. Contamination-Free Manufacturing for Semiconductors and Other Precision Products; Taylor \& Francis, 2001.

(78) Yablonovitch, E.; Allara, D. L.; Chang, C. C.; Gmitter, T.; Bright, T. B. Physical Review Letters 1986, 57, 249-252.

(79) Abbink, H. C.; Broudy, R. M.; McCarthy, G. P. Journal of Applied Physics 1968, 39, 4673-4681. 
(80) Ruan, C.-Y.; Lobastov, V. A.; Vigliotti, F.; Chen, S.; Zewail, A. H. Science 2004, 304, 80-84.

(81) Gosele, U.; Stenzel, H.; Martini, T.; Steinkirchner, J.; Conrad, D.; Scheerschmidt, K. Applied Physics Letters 1995, 67, 3614-3616.

(82) Derycke, V.; Soukiassian, P. G.; Amy, F.; Chabal, Y. J.; D'Angelo, M. D.; Enriquez, H. B.; Silly, M. G. Nat Mater 2003, 2, 253-258.

(83) Silvestrelli, P. L.; Toigo, F.; Ancilotto, F. The Journal of Physical Chemistry B 2006, 110, 12022-12028.

(84) Bal, J. K.; Kundu, S.; Hazra, S. Physical Review B 2010, 81, 045404.

(85) Weinberger, B. R.; Peterson, G. G.; Eschrich, T. C.; Krasinski, H. A. Journal of Applied Physics 1986, 60, 3232-3234.

(86) Ubara, H.; Imura, T.; Hiraki, A. Solid State Communications 1984, 50, 673-675.

(87) Burrows, V. A.; Chabal, Y. J.; Higashi, G. S.; Raghavachari, K.; Christman, S. B. Applied Physics Letters 1988, 53, 998-1000.

(88) Chabal, Y. J.; Higashi, G. S.; Raghavachari, K.; Burrows, V. A. Journal of Vacuum Science \& Technology A: Vacuum, Surfaces, and Films 1989, 7, 2104-2109.

(89) Linford, M. R.; Chidsey, C. E. D. Journal of the American Chemical Society 1993, 115, 12631-12632.

(90) Linford, M. R.; Fenter, P.; Eisenberger, P. M.; Chidsey, C. E. D. Journal of the American Chemical Society 1995, 117, 3145-3155.

(91) Bansal, A.; Li, X.; Lauermann, I.; Lewis, N. S.; Yi, S. I.; Weinberg, W. H. Journal of the American Chemical Society 1996, 118, 7225-7226.

(92) Lee, H. H. Fundamentals of microelectronics processing; McGraw-Hill, 1990.

(93) Manos, D. M.; Flamm, D. L. Plasma Etching: An Introduction; Acad. Press, 1989.

(94) Vossen, J. L. Journal of Physics E: Scientific Instruments 1979, 12, 159.

(95) Miura, T.-a.; Niwano, M.; Shoji, D.; Miyamoto, N. Journal of Applied Physics 1996, 79, 4373-4380.

(96) Niwano, M.; Kageyama, J.-i.; Kurita, K.; Kinashi, K.; Takahashi, I.; Miyamoto, N.

Journal of Applied Physics 1994, 76, 2157-2163.

(97) Saddow, S. Silicon Carbide Biotechnology: A Biocompatible Semiconductor for

Advanced Biomedical Devices and Applications; Elsevier Science, 2011.

(98) Seu, K. J.; Pandey, A. P.; Haque, F.; Proctor, E. A.; Ribbe, A. E.; Hovis, J. S.

Biophysical journal 2007, 92, 2445-2450.

(99) Yan, M.; Harnish, B. Advanced Materials 2003, 15, 244-248.

(100) Yan, M.; Bartlett, M. A. Nano Letters 2002, 2, 275-278.

(101) Demirel, A. L.; Granick, S. The Journal of Chemical Physics 2001, 115, 1498-1512.

(102) Mate, C. M.; McClelland, G. M.; Erlandsson, R.; Chiang, S. Physical Review

Letters 1987, 59, 1942-1945.

(103) Israelachvili, J. N.; Adams, G. E. Journal of the Chemical Society, Faraday

Transactions 1: Physical Chemistry in Condensed Phases 1978, 74, 975-1001.

(104) Betzig, E.; Finn, P. L.; Weiner, J. S. Applied Physics Letters 1992, 60, 2484-2486.

(105) Antognozzi, M.; Haschke, H.; Miles, M. J. Review of Scientific Instruments 2000,

71, 1689-1694. 
(106) Hsu, J. W. P.; Lee, M.; Deaver, B. S. Review of Scientific Instruments 1995, 66, 3177-3181.

(107) Barenz, J.; Hollricher, O.; Marti, O. Review of Scientific Instruments 1996, 67, 1912-1916.

(108) Lee, M.; McDaniel, E. B.; Hsu, J. W. P. Review of Scientific Instruments 1996, 67, 1468-1471.

(109) Rosa, A. L.; Cui, X.; McCollum, J.; Li, N.; Nordstrom, R. Review of Scientific Instruments 2005, 76, 093707.

(110) Castellanos-Gomez, A.; Agraït, N.; Rubio-Bollinger, G. Nanotechnology 2010, 21, 145702.

(111) Wei, P. K.; Fann, W. S. Journal of Applied Physics 1998, 83, 3461-3468.

(112) Shekhawat, G. S.; Briggs, G. A.; Kolosov, O. V.; Geer, R. E. In Characterization and Metrology for ULSI Technology 2000; Seiler, D. G., Diebold, A. C., Shaffner, T. J., McDonald, R., Bullis, W. M., Smith, P. J., Secula, E. M., Eds. 2000; Vol. 550, p 449-451. (113) Granick, S. Science 1991, 253, 1374-1379.

(114) Antognozzi, M.; Humphris, A. D. L.; Miles, M. J. Applied Physics Letters 2001, 78, 300-302.

(115) Segur, J. B.; Oberstar, H. E. Industrial \& Engineering Chemistry 1951, 43, 21172120.

(116) Friedt, J.-M.; Carry, E. American Journal of Physics 2007, 75, 415-422.

(117) Christen, M. Sensors and Actuators 1983, 4, 555-564.

(118) Dube, D. H.; Bertozzi, C. R. Nat. Rev. Drug. Discov. 2005, 4, 477-488.

(119) Fuster, M. M.; Esko, J. D. Nature Reviews: Cancer 2005, 5, 526-542.

(120) Liu, F.-T.; Rabinovich, G. A. Nature Reviews: Cancer 2005, 5, 29-41.

(121) Szymanski, C. M.; Wren, B. W. Nature Reviews: Microbiology 2005, 3, 225-237.

(122) Ohtsubo, K.; Marth, J. D. Cell 2006, 126, 855-867.

(123) Sharon, N. Biochim. Biophys. Acta, Gen. Subj. 2006, 1760, 527-537.

(124) Crocker, P. R.; Paulson, J. C.; Varki, A. Nature Reviews: Immunology 2007, 7, 255-266.

(125) Poveda, A.; Jimenez-Barbero, J. Chemical Society Reviews 1998, 27, 133-143.

(126) Beccati, D.; Halkes, K. M.; Batema, G. D.; Guillena, G.; Carvalho de Souza, A.; van Koten, G.; Kamerling, J. P. ChemBioChem 2005, 6, 1196-1203.

(127) Smith, E. A.; Thomas, W. D.; Kiessling, L. L.; Corn, R. M. Journal of the American Chemical Society 2003, 125, 6140-6148.

(128) von Itzstein, M.; Colman, P. Current Opinion in Structural Biology 1996, 6, 703709.

(129) Fernández-Alonso, M. d. C.; Cañada, F. J.; Jiménez-Barbero, J.; Cuevas, G.

Journal of the American Chemical Society 2005, 127, 7379-7386.

(130) Clarke, C.; Woods, R. J.; Gluska, J.; Cooper, A.; Nutley, M. A.; Boons, G.-J.

Journal of the American Chemical Society 2001, 123, 12238-12247.

(131) Lee, Y. C. Journal of Biochemistry 1997, 121, 818-825.

(132) Dupres, V.; Verbelen, C.; Dufrene, Y. F. Biomaterials 2007, 28, 2393-2402.

(133) Hinterdorfer, P.; Dufrene, Y. F. Nature Methods 2006, 3, 347-355.

(134) Neuman, K. C.; Nagy, A. Nat Meth 2008, 5, 491-505. 
(135) Strunz, T.; Oroszlan, K.; Schäfer, R.; Güntherodt, H.-J. PNAS 1999, 96, 1127711282.

(136) Yan, M.; Ren, J. Chemistry of Materials 2004, 16, 1627-1632.

(137) Yan, M. Chemistry- A European Journal 2007, 13, 4138-4144.

(138) Gann, J. P.; Yan, M. Langmuir 2008, 24, 5319-5323.

(139) Wang, X.; Ramström, O.; Yan, M. Journal of Materials Chemistry 2009, 19, 89448949.

(140) Wang, X.; Liu, L. H.; Ramstrom, O.; Yan, M. Experimental Biology and Medicine 2009, 234, 1128-1139.

(141) Liu, L.-H.; Yan, M. Accounts of Chemical Research 2010, 43, 1434-1443.

(142) Pei, Y.; Yu, H.; Pei, Z.; Theurer, M.; Ammer, C.; André, S.; Gabius, H.-J.; Yan, M.; Ramström, O. Analytical Chemistry 2007, 79, 6897-6902.

(143) Pei, Z.; Yu, H.; Theurer, M.; Waldén, A.; Nilsson, P.; Yan, M.; Ramström, O.

ChemBioChem 2007, 8, 166-168.

(144) Bittiger, H.; Schnebli, H. P. Concanavalin A as a Tool; John Wiley and Sons:

London, 1976.

(145) Lis, H.; Sharon, N. Chemical Reviews 1998, 98, 637-674.

(146) Wang, H.; Ren, J.; Hlaing, A.; Yan, M. Journal of Colloid and Interface Science

2011, 354, 160-167.

(147) Madwar, C.; Chu Kwan, W.; Deng, L.; Ramström, O.; Schmidt, R.; Zou, S.; Cuccia, L. A. Langmuir 2010, 26, 16677-16680.

(148) Ratto, T. V.; Langry, K. C.; Rudd, R. E.; Balhorn, R. L.; Allen, M. J.; McElfresh, M. W. Biophysical Journal 2004, 86, 2430-2437.

(149) Schwarz, F. P.; Puri, K. D.; Bhat, R. G.; Surolia, A. Journal of Biological Chemistry 1993, 268, 7668-7677.

(150) Tyagi, A.; Wang, X.; Deng, L.; Ramström, O.; Yan, M. Biosensors and Bioelectronics 2010, 26, 344-350.

(151) Lis, H.; Sharon, N. Chemical Reviews 1998, 98, 637-674.

(152) Piner, R. D.; Zhu, J.; Xu, F.; Hong, S. H.; Mirkin, C. A. Science 1999, 283, 661663.

(153) Lee, K. B.; Park, S. J.; Mirkin, C. A.; Smith, J. C.; Mrksich, M. Science 2002, 295, 1702-1705.

(154) Lim, J. H.; Mirkin, C. A. Advanced Materials 2002, 14, 1474-1477.

(155) Wang, H. T.; Nafday, O. A.; Haaheim, J. R.; Tevaarwerk, E.; Amro, N. A.;

Sanedrin, R. G.; Chang, C. Y.; Ren, F.; Pearton, S. J. Applied Physics Letters 2008, 93, 143105.

(156) Maynor, B. W.; Li, Y.; Liu, J. Langmuir 2001, 17, 2575-2578.

(157) Demers, L. M.; Ginger, D. S.; Park, S. J.; Li, Z.; Chung, S. W.; Mirkin, C. A.

Science 2002, 296, 1836-1838.

(158) Ivanisevic, A.; Mirkin, C. A. Journal of the American Chemical Society 2001, 123, 7887-7889.

(159) Huang, C. Y.; Jiang, G. Q.; Advincula, R. Macromolecules 2008, 41, 4661-4670.

(160) Maynor, B. W.; Li, J. Y.; Lu, C. G.; Liu, J. Journal of the American Chemical Society 2004, 126, 6409-6413. 
(161) Anderson, M. S. Analytical Chemistry 2005, 77, 2907-2911.

(162) Yang, M.; Sheehan, P. E.; King, W. P.; Whitman, L. J. Journal of the American Chemical Society 2006, 128, 6774-6775.

(163) Cui, Y.; Wei, Q. Q.; Park, H. K.; Lieber, C. M. Science 2001, 293, 1289-1292.

(164) Rozhok, S.; Piner, R.; Mirkin, C. A. Journal of Physical Chemistry B 2003, 107, 751-757.

(165) Hong, S. H.; Zhu, J.; Mirkin, C. A. Langmuir 1999, 15, 7897-7900.

(166) Harnish, B.; Robinson, J. T.; Pei, Z. C.; Ramstrom, O.; Yan, M. D. Chemistry of Materials 2005, 17, 4092-4096.

(167) Tam, T. K.; Ornatska, M.; Pita, M.; Minko, S.; Katz, E. Journal of Physical Chemistry C 2008, 112, 8438-8445.

(168) Privman, M.; Tam, T. K.; Pita, M.; Katz, E. Journal of the American Chemical Society 2009, 131, 1314-1321.

(169) Vaganova, E.; Meshulam, G.; Kotler, Z.; Rozenberg, M.; Yitzchaik, S. Journal of Fluorescence 2000, 10, 81-88.

(170) Sader, J. E.; Chon, J. W. M.; Mulvaney, P. Review of Scientific Instruments 1999, 70, 3967-3969.

(171) Maedler, C.; Chada, S.; Cui, X.; Taylor, M.; Yan, M.; La Rosa, A. Journal of Applied Physics 2008, 104, 014311.

(172) Lyuksyutov, S. F.; Paramonov, P. B.; Juhl, S.; Vaia, R. A. Applied Physics Letters 2003, 83, 4405-4407.

(173) Lyuksyutov, S. F.; Vaia, R. A.; Paramonov, P. B.; Juhl, S.; Waterhouse, L.; Ralich, R. M.; Sigalov, G.; Sancaktar, E. Nature Materials 2003, 2, 468-472.

(174) Cho, N.; Ryu, S.; Kim, B.; Schatz, G. C.; Hong, S. H. Journal of Chemical Physics 2006, 124, 024714.

(175) Li, Y.; Maynor, B. W.; Liu, J. Journal of the American Chemical Society 2001, 123, 2105-2106.

(176) Weeks, B. L.; Noy, A.; Miller, A. E.; De Yoreo, J. J. Physical Review Letters 2002, 88, 255505.

(177) Jang, J. Y.; Hong, S. H.; Schatz, G. C.; Ratner, M. A. Journal of Chemical Physics 2001, 115, 2721-2729.

(178) Jang, J. Y.; Schatz, G. C.; Ratner, M. A. Journal of Chemical Physics 2002, 116, 3875-3886. 